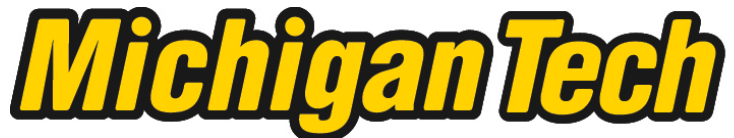 \\ Michigan Technological University Create the Future Digital Commons @ Michigan Tech
}

Remote sensing of volcanic plumes using the Advanced Spaceborne Thermal Emission and Reflection Radiometer (ASTER).

Lorna Alison Henney

Michigan Technological University

Follow this and additional works at: https://digitalcommons.mtu.edu/etds

Part of the Geology Commons

Copyright 2012 Lorna Alison Henney

\section{Recommended Citation}

Henney, Lorna Alison, "Remote sensing of volcanic plumes using the Advanced Spaceborne Thermal Emission and Reflection Radiometer (ASTER).", Dissertation, Michigan Technological University, 2012.

https://doi.org/10.37099/mtu.dc.etds/323

Follow this and additional works at: https://digitalcommons.mtu.edu/etds

Part of the Geology Commons 


\title{
REMOTE SENSING OF VOLCANIC PLUMES USING THE ADVANCED SPACEBORNE THERMAL EMISSION AND REFLECTION RADIOMETER (ASTER).
}

By

Lorna Alison Henney

\author{
A DISSERTATION \\ Submitted in partial fulfillment of the requirements for the degree of \\ DOCTOR OF PHILOSOPHY \\ (Geology) \\ MICHIGAN TECHNOLOGICAL UNIVERSITY \\ 2012 \\ (C2012 Lorna Alison Henney
}


This dissertation, "Remote Sensing of Volcanic Plumes using the Advanced Spaceborne Thermal Emission and Reflection Radiometer (ASTER),"is hereby approved in partial fulfillment of the requirements for the Degree of DOCTOR OF PHILOSOPHY IN GEOLOGY

Department of Geological and Mining Engineering and Sciences

Signatures:

Dissertation Advisor

Matthew Watson

Department Chair

Wayne Pennington

Date 
For Mum, Dad and Fergus 


\section{Table of Contents}

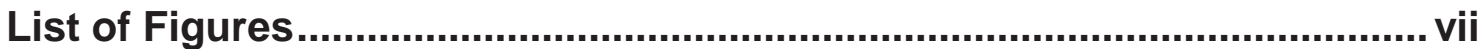

List of Tables ................................................................................................ xi

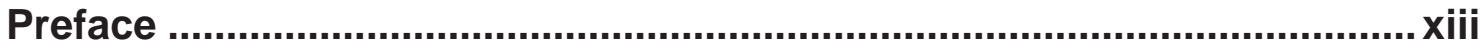

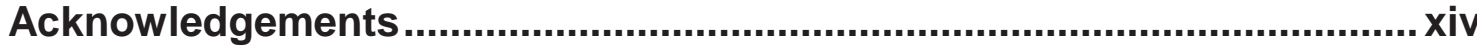

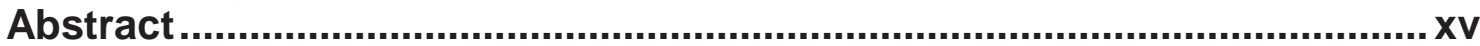

Chapter 1:Remote Sensing of Passively Degassing Volcanoes 1

1.1. Introduction.............................................................................................2

1.1.2. Sulfur Dioxide Emissions from Passively Degassing Volcanoes ......2

1.1.2. Satellite Remote Sensing.................................................................... 4

1.1.3. Previous Estimations of Global Volcanic $\mathrm{SO}_{2}$ Emissions in to the

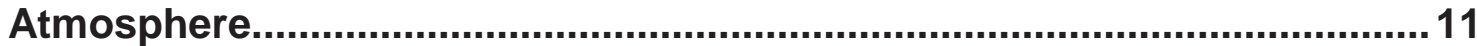

1.1.4. Geologic Setting and Target Volcanoes ...........................................13

1.2. Methodology-Sensor Descriptions and Algorithms ............................17

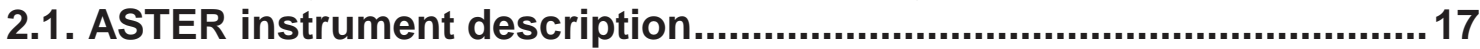

2.2. ASTER TIR retrieval Algorithm (MAP_SO2) .......................................18

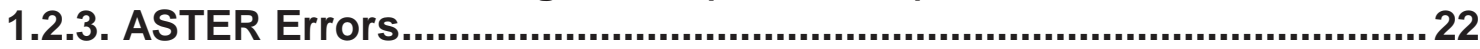

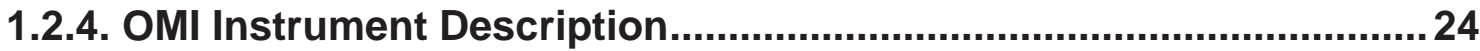

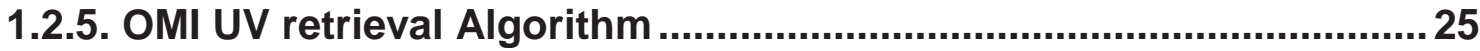

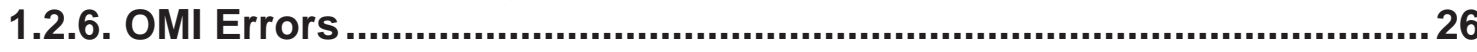

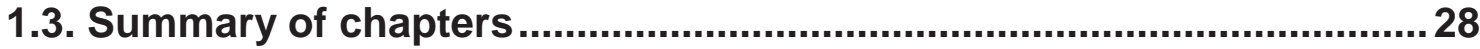

Chapter 2: A Comparison of $\mathrm{SO}_{2}$ Retrival Techniques using Mini UV Spectrometers and ASTER Imagery at Lascar Volcano,

Chile ........................................................................................................... 30

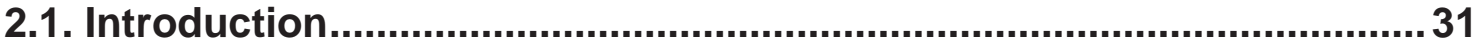

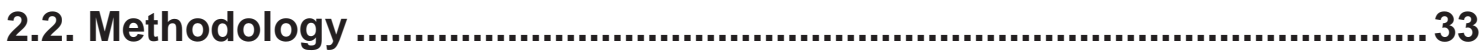

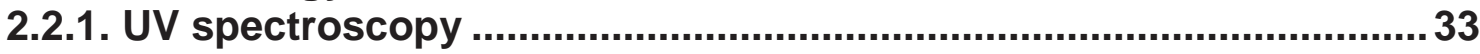

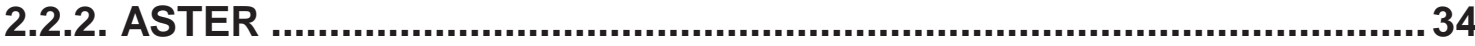

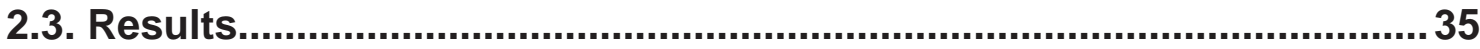

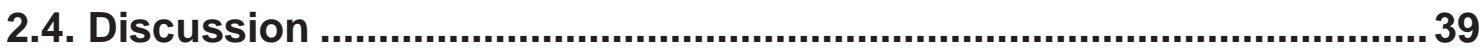

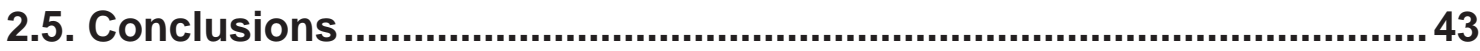

Chapter 3: Visual Observations of Volcanic Plumes using Principal Components Analysis and Decorrelation Stretch Enhancements on ASTER Thermal Infrared Data .......................... 44

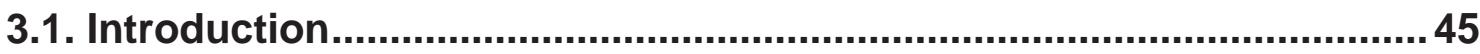

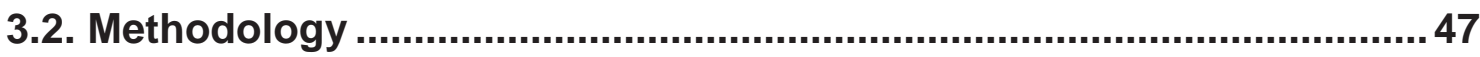


3.2.1. Principal Component Analysis ............................................................ 47

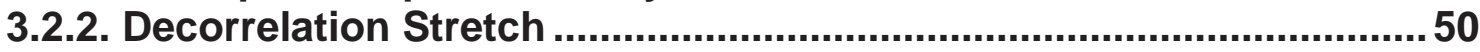

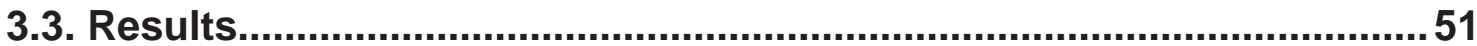

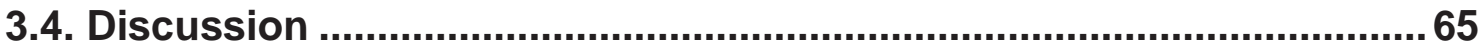

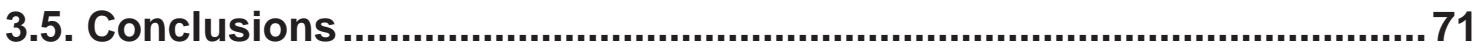

Chapter 4: An investigation into the activity at Lascar volcano during 2000-2011, using the Advanced Spaceborne Thermal Emission and Reflection Radiometer (ASTER) ............................72

4.1. Introduction......................................................................................73

4.1.1. Lascar Volcano and Geological History ...........................................75

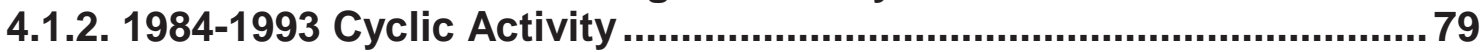

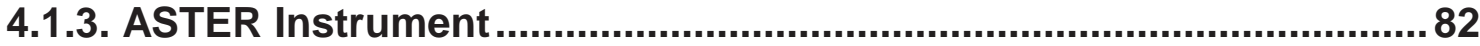

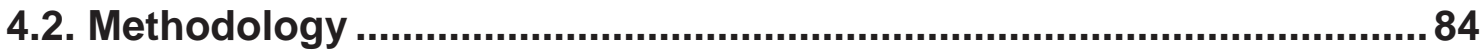

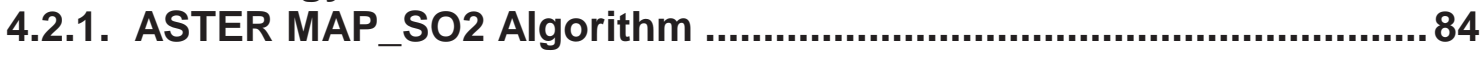

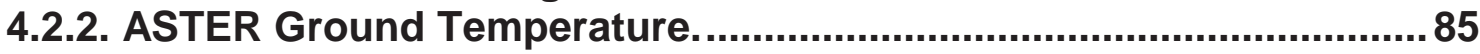

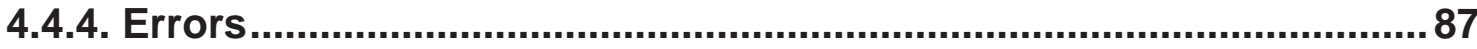

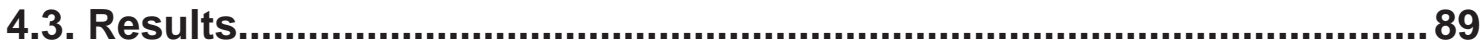

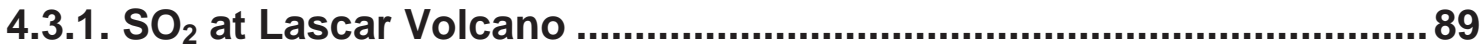

4.3.2. Maximum crater temperature at Lascar........................................... 108

4.4.1. Changes in $\mathrm{SO}_{2}$ emissions and Hotspot Temperatures ................110

4.5. Conclusions and Further Work ........................................................117

Chapter 5 An Estimate of Volcanic $\mathrm{SO}_{2}$ Emissions into the Troposphere from the Central and South American Volcanic Arcs. .................................................................................................119

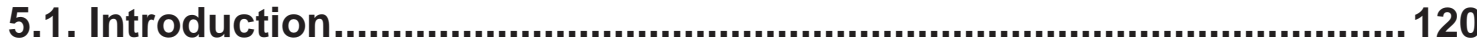

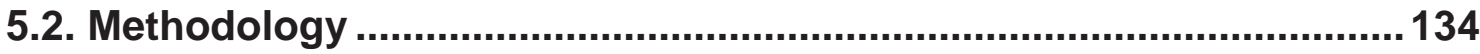

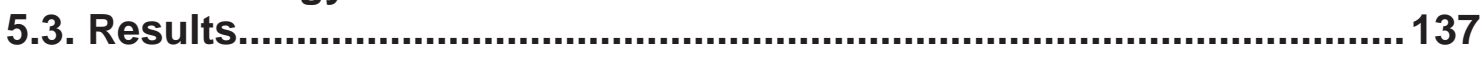

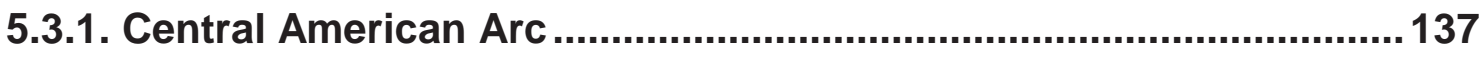

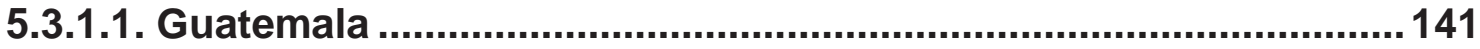

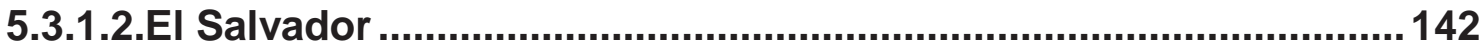

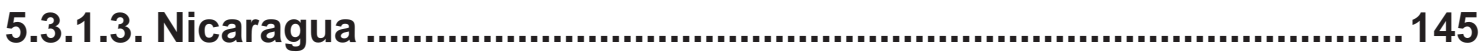

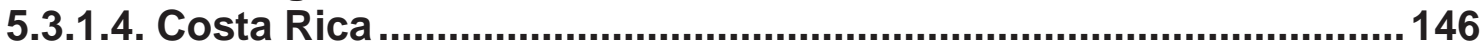

5.3.2. South American Volcanic Arc .........................................................147

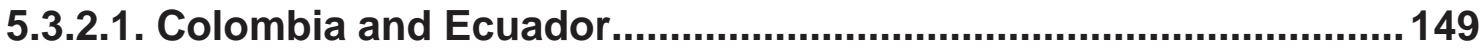

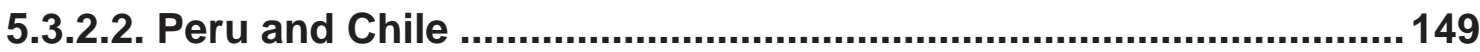

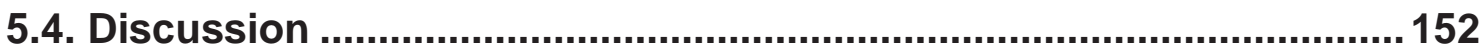

5.4.1. Comparison to Previous Estimates ..............................................152

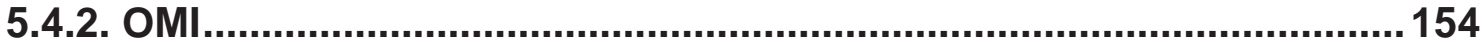

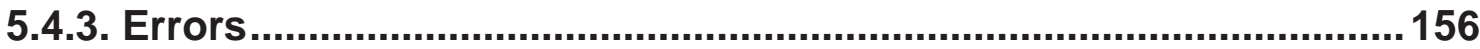

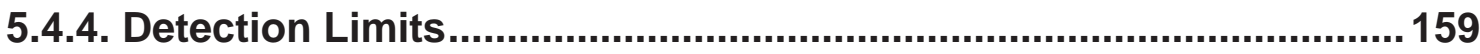

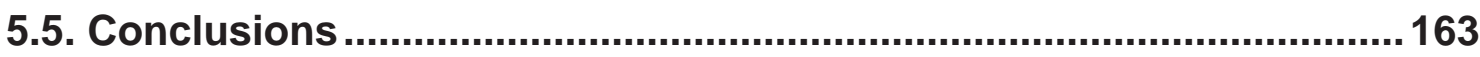


Chapter 6 Summary and Conclusions ….........................................166

6. Summary and Conclusions .................................................................167

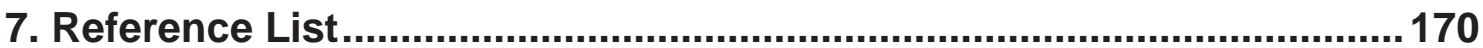

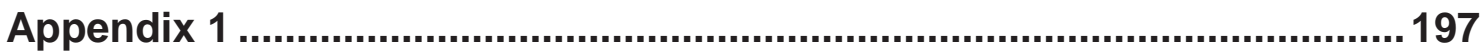

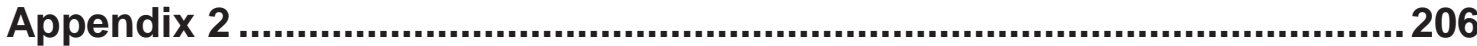

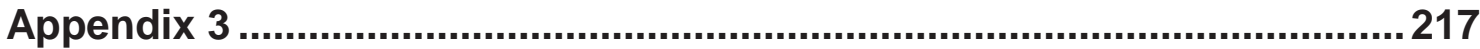

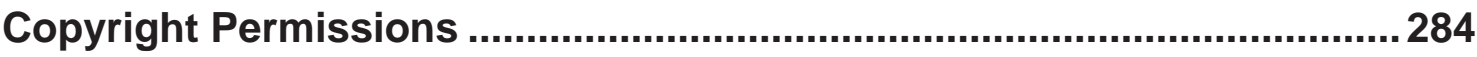




\section{List of Figures}

Figure 1.1: Map of Central (a) and South America (b), illustrating the major volcanoes

Figure 1.2: ASTER's spectral response functions for the TIR channels (a), and the $8.6 \mu \mathrm{m} \mathrm{SO}{ }_{2}$ absorption feature (b).

Figure 2.1: 1a illustrates Lascar volcano with the location of where the MUSe measurements were taken, and the direction of the plume, labeled. $1 \mathrm{~b}$ is the TIR ASTER image (bands 10, 11 and 12) of Lascar volcano for December $7^{\text {th }}, 2004$

Figure 2.2: The Map_SO2 image produced from the ASTER data. The location of the eighteen transects taken across the plume is illustrated......36

Figure 2.3: A timeline of $\mathrm{SO}_{2}$ flux data for the MUSe and ASTER instruments. The dashed line indicates when the ASTER image was acquired. Figure $3 \mathrm{~b}$ focuses on the ASTER portion of the timeline to show the actual data points with their associated errors. 38

Figure 2.4: Average $\mathrm{SO}_{2}$ fluxes determined using the MUSe and ASTER instruments. The $\mathrm{SO}_{2}$ flux for ASTER transect 1 is also displayed.41

Figure 2.5: Average $\mathrm{SO}_{2}$ fluxes determined using the MUSe and ASTER instruments. The $\mathrm{SO}_{2}$ flux for ASTER transect 1 is also displayed.42

Figure 3.1: Chaiten, Chile, 05-31-2008, TIR ASTER image (a), PCA (b) and DCS (c).

Figure 3.2: Eyjafjallokull, Iceland, 04-17-2010, TIR ASTER image (a), PCA (b) and DCS (c) .

Figure 3.3: Ubinas, Peru, 05-08-2006, TIR ASTER image (a), PCA (b) and DCS (c) 60 
Figure 3.4: Popocatepetl, Mexico, 12-15-2007, TIR ASTER image (a), PCA (b) and DCS (c)

Figure 3.5: Turrialba, Costa Rica, 01-31-2011, TIR ASTER image (a), PCA (b) and DCS (c)

Figure 3.6: Lascar, Chile, 12-07-2004, TIR ASTER image (a), PCA (b) and DCS (c) 63

Figure 3.7: Masaya, Nicaragua, 02-09-2005, TIR ASTER image (a), PCA (b) and DCS (c) 64

Figure 3.8: Data distribution for the ASTER TIR images of Chaiten, Lascar and Ubinas 67

Figure 3.9: Kileaua, U.S.A., 03-23-2008, TIR ASTER image (a), PCA (b) and DCS (c) 70

Figure 4.1: (a) Location of Lascar volcano. (b) Near Visible Infrared image of Lascar highlighting the active crater. 77

Figure 4.2: Geology map of Lascar (Gardeweg et al.1998) 78

Figure 4.3: Timeline of activity at Lascar including gas emissions, hotspot data and eruptions 106

Figure 4.4: Examples of OMI image of plumes from Lascar from 4 May 2005 and 27 Aug 2009 107

Figure 4.5: The ASTER temperatures produced by Murphy et al. (2011) in comparison to our temperatures produced from the ASTER data...111

Figure 5.1: Location map of the volcanoes in the Central and South American volcanic arcs. 124 
Figure 5.2: Flowchart illustrating the process of selecting an image to be analyzed by the MAP_SO2 algorithm 136

Figure 5.3: $\mathrm{PCA}$ and DCS enhancements of a plume from Ubinas volcano, Peru, 05-08-2006. 136

Figure 5.4: Examples of OMI images of plumes from Turrialba, in the period 2009-2010. 139

Figure 5.5: Map of Central America with sample ASTER images. Each image illustrates the $\mathrm{SO}_{2}$ map and the image prior to processing. 140

Figure 5.6: OMI images of plumes produced during the 2005 eruptive episode at Santa Ana. 144

Figure 5.7: Plumes observed by OMI at Ubinas, Peru 151

Figure 5.8: Plot of Log of volcano frequency versus $\mathrm{Log}$ of $\mathrm{SO}_{2}$ emissions illustrating the value of constant $\mathrm{c}$.... 158

Figure B.1: PCA and DCS enhancements of Arenal, Costa Rica..............207

Figure B.2: PCA and DCS enhancements of Bezmianny, Russia............207

Figure B.3: PCA and DCS enhancements of Bezmianny, Russia.............208

Figure B.4: PCA and DCS enhancements of Chaiten, Chile..................208

Figure B.5: PCA and DCS enhancements of Eyjallajokul, Iceland............209

Figure B.6: PCA and DCS enhancements of Kilauea, USA …....................210

Figure B.7: PCA and DCS enhancements of Lascar, Chile …..................... 210 
Figure B.8: PCA and DCS enhancements of Pacaya, Guatemala 211

Figure B.9: PCA and DCS enhancements of Pacaya, Guatemala 211

Figure B.10: PCA and DCS enhancements of Pacaya, Guatemala 212

Figure B.11: PCA and DCS enhancements of Pacaya, Guatemala.............. 213

Figure B.12: PCA and DCS enhancements of Planchon-petroa, Chile ......... 215

Figure B.13: PCA and DCS enhancements of Tungurahua, Ecuador 215

Figure B.14: PCA and DCS enhancements of Ubinas......................216 


\section{List of Tables}

Table 1.1: ASTER subsystem characteristics......................................... 7

Table 3.1: Examples of loading factors from the key images discussed in the results section

Table 3.2: A description of the plumes enhanced by the DCS and PCA.........52

Table 4.1: TIR, PCA and $\mathrm{SO}_{2}$ maps produced from the ASTER data of Lascar Volcano, Chile

Table 4.2: $\mathrm{SO}_{2}$ maps of Lascar where only $\mathrm{SO}_{2}$ gathered in the crater and no detectable plume was produced

Table4.3: Dates of the eruptions at Lascar and eruption column heights above the vent. 105

Table 5.1: Previous estimates of global $\mathrm{SO}_{2}$ emissions from volcanoes and the technique used. The estimates include passive and eruptive emissions

Table 5.2: Description of several of the volcanoes in the Central and South American volcanic arcs.

Table 5.3: Estimate of $\mathrm{SO}_{2}$ emissions from volcanoes in the Central American Volcanic arc (2000-2010). The estimate is broken down in to individual volcanoes and by country....

Table 5.4: $\mathrm{SO}_{2}$ fluxes for South American volcanoes (2000-2010) organized by country and a breakdown of averages calculated by each method 140

Table 5.5: Average daily $\mathrm{SO}_{2}$ emissions from OMI compared to other sources of data at volcanoes where $\mathrm{OMI}$ detected $\mathrm{SO}_{2}$ plumes

Table 5.6: Volcanoes studied and average $\mathrm{SO}_{2}$ emissions detected from them using ASTER

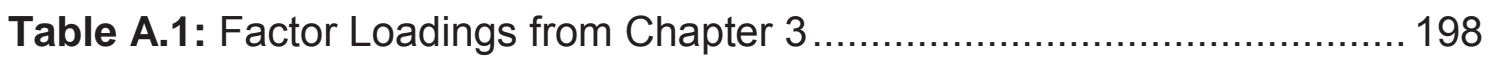

Table C.1: ASTER and ground based $\mathrm{SO}_{2}$ measurements.......................218 


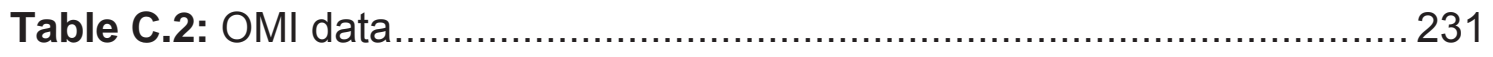

Table C.3: OMI data for Colombia and Ecuador .................................. 268 


\section{Preface}

Chater 2 was published in the Bulletin of Volcanology and was authored by Lorna Henney, Lizzette Rodriguez and Matthew Watson. My contribution to the paper was the analysis of the ASTER data as well as the interpretation of our results. 


\section{Acknowledgements}

I am profoundly grateful for the endless help, support, and guidance from my advisor and committee members, Matt Watson, Lizzette Rodriguez, Ann Maclean and Simon Carn. Without their comments and encouragement I would not have been able to get through my PhD. Without generous funding from Matt Watson, Michigan Technological University department of Geological Mining Engineering Sciences and my parents, this research would have not been possible and I would not have had the opportunity to be a teaching assistant and go to conferences. I would also like to thank Kelly McLean and Amie Ledgerwood with all the logistical support and girl talk over the years. Most importantly I would like to thank my family and the friends I have made over the past six years who have provided constant love and support. 


\section{Abstract}

The Advanced Spaceborne Thermal Emission and Reflection Radiometer (ASTER) has been used to quantify $\mathrm{SO}_{2}$ emissions from passively degassing volcanoes. This dissertation explores ASTER's capability to detect $\mathrm{SO}_{2}$ with satellite validation, enhancement techniques and extensive processing of images at a variety of volcanoes. ASTER is compared to the Mini UV Spectrometer (MUSe), a ground based instrument, to determine if reasonable $\mathrm{SO}_{2}$ fluxes can be quantified from a plume emitted from Lascar, Chile. The two sensors were in good agreement with ASTER proving to be a reliable detector of $\mathrm{SO}_{2}$. ASTER illustrated the advantages of imaging a plume in $2 \mathrm{D}$, with better temporal resolution than the MUSe. $\mathrm{SO}_{2}$ plumes in ASTER imagery are not always discernible in the raw TIR data. Principal Component Analysis (PCA) and Decorrelation Stretch (DCS) enhancement techniques were compared to determine how well they highlight a variety of volcanic plumes. DCS produced a consistent output and the composition of the plumes was easy to identify from explosive eruptions. As the plumes became smaller and lower in altitude they became harder to distinguish using DCS. PCA proved to be better at identifying smaller low altitude plumes. ASTER was used to investigate $\mathrm{SO}_{2}$ emissions at Lascar, Chile. Activity at Lascar has been characterized by cyclic behavior and persistent degassing (Matthews et al. 1997). Previous studies at Lascar have primarily focused on changes in thermal infrared anomalies, neglecting gas emissions. Using the $\mathrm{SO}_{2}$ data along with changes in thermal anomalies and visual observations it is evident that Lascar is at the end an eruptive cycle that began in 1993. Declining gas emissions and crater temperatures suggest that the conduit is sealing. ASTER and the Ozone Monitoring Instrument (OMI) were used to determine the annual contribution of $\mathrm{SO}_{2}$ to the troposphere from the Central and South American volcanic arcs between 2000 and 2011. Fluxes of $3.4 \mathrm{Tg} / \mathrm{a}$ for Central America and 3.7 Tg/a for South America were calculated. The detection limits of ASTER were explored. The results a proved to be 
interesting, with plumes from many of the high emitting volcanoes, such as Villarrica, Chile, not being detected by ASTER. 
Chapter 1

\section{Remote Sensing of Passively Degassing Volcanoes}




\subsection{Introduction}

\subsubsection{Sulfur Dioxide Emissions from Passively Degassing Volcanoes}

Sulfur dioxide is the third most abundant gas emitted from volcanoes, after water vapor and carbon dioxide. Background levels of $\mathrm{SO}_{2}$ in the troposphere are low in comparison to $\mathrm{H}_{2} \mathrm{O}$ and $\mathrm{CO}_{2}$ (Delmelle and Stix 2000), making it an ideal species to monitor through the use of remote sensing techniques. Regular and consistent quantification of volcanogenic $\mathrm{SO}_{2}$ is important for several reasons.

Firstly $\mathrm{SO}_{2}$ can have an impact on the local population and environment by destroying crops through acidification, contaminating the water supply and causing health problems, particularly the respiratory system (Baxter et al. 1982; Allen et al. 2000; Delmelle et al. 2001; Delmelle 2003).

Second, constant $\mathrm{SO}_{2}$ measurements at volcanoes allow baseline averages to be established during quiescent non-eruptive phases of activity. Distinct patterns of activity can be recognized and related to changes in volcanic activity and eruptive style. These deviations in the $\mathrm{SO}_{2}$ baseline flux can provide an indication of what is happening in the subsurface system of a volcano (Symonds et al. 1994).

Third, $\mathrm{SO}_{2}$ emitted into the atmosphere can perturb the climate. $\mathrm{SO}_{2}$ is converted to sulfate aerosol, which can have a residence time of greater than 2 years in the stratosphere (Robock 2000). Aerosols in the atmosphere can reduce the quantity of incoming shortwave radiation reaching the Earth's surface by scattering it back in to space. Thus volcanoes that inject large quantities of $\mathrm{SO}_{2}$ into the atmosphere can cause global cooling (Robock 2000). 
The quantity of natural $\mathrm{SO}_{2}$ injected into the atmosphere is significant in evaluating the impacts it has on the atmosphere and when modeling anthropogenic climate change. It is well-known that large eruptions inject huge quantities of $\mathrm{SO}_{2}$ into the atmosphere, which are easily detected and accounted for. However, many volcanoes are persistently degassing during non-eruptive episodes. Volcanoes contribute a large portion of the total input of global volcanic gas into the atmosphere (Horrocks et al. 2003) with $\mathrm{SO}_{2}$ estimates ranging from 1.5 to $50 \mathrm{Tg} / \mathrm{a}$ (Andres and Kasgnoc 1998).

As an example, extensive data sets of $\mathrm{SO}_{2}$ emissions have been collected at volcanoes such as Mt Etna, Sicily, and Stromboli, Italy. These data have been linked to subsurface activity indicating when the volcanic system is going to potentially produce eruptive behavior months in advance (Caltabiano et al. 2004). Work carried out at Mt Etna monitoring gas emissions between 19872000 by Caltabiano et al. (2004), showed how these data can be used for monitoring purposes. Data were collected at least once a week until 1996 when measurements were taken 2-3 times a week. It was noted that $\mathrm{SO}_{2}$ emissions ranged from 600 to $25,000 \mathrm{t} \mathrm{d}^{-1}$. Low values of $\mathrm{SO}_{2}$ were recorded and coincided with deep seismicity in advance of eruptions. Increasing trends are used to infer the movement of new magma in to the shallower parts of the volcanic systems. Decreasing trends of $\mathrm{SO}_{2}$ signify the progressive degassing of magma batches in the shallow system.

At Stromboli, gas emissions have been investigated by Allard et al. (1994), Allard (1997) and Burton et al. (2009). The results in all these studies linked the $\mathrm{SO}_{2}$ output to what was happening in the shallow regions of the volcano. At Stromboli, it is evident that the gas flux is high with fluxes range from 130 to $1500 \mathrm{t} \mathrm{d}^{-1}$ (Mallinconico 1987; Allard et al. 1994; Weibring et al. 1998; 2002; Burton et al. 2003) during quiescent periods. However, there is a low output of magma, and explosive activity is infrequent (Allard et al. 1994). During short 
eruptive episodes, the gas flux increases. Allard et al. (1994) demonstrated that such large emissions of $\mathrm{SO}_{2}$ indicated that there was a continuous supply of new under gassed magma to the shallow regions of the volcanic system from a great depth. Since little magma is erupted from the volcano, the degassed magma must be removed through convective processes and stored at an upper crustal level. More recent work carried out by Burton et al. (2009) illustrated the importance of automated $\mathrm{SO}_{2}$ measurements from the FLux Automatic MEasurement (FLAME) network. During 2007, a period of elevated activity at Stromboli took place with an eruption. Measurements were recorded continuously before, during, and after this eruptive episode. The gas emissions were seen to increase prior to the eruption, but just before the eruption, the gas emissions drop. Throughout the eruption, $\mathrm{SO}_{2}$ is elevated to around 4 times the average emission rate. After the eruption ends, the $\mathrm{SO}_{2}$ emission rate slowly decreases over time. Such trends in the emission rate have been seen before at Stromboli and can be explained by a model proposed by Burton et al. (2003), where magma flowing into the shallow volcanic system produces percolation pathways in which gas from the new magma can escape up quickly and easily. The degassed magma descends back down the conduit. These studies at Etna and Stromboli illustrate the importance of constant monitoring of volcanic gases in terms of processes within the volcano and to predict impending eruptions possibly up to several months in advance.

\subsubsection{Satellite Remote Sensing}

Satellite based remote sensing techniques have become an important tool for investigating volcanic activity over the past few decades (Carn et al. 2003). Much work has been carried out in order to improve the detection of volcanic emissions. In 1982, El Chichon, Mexico erupted injecting $3.3 \times 10^{9} \mathrm{~kg}$ of $\mathrm{SO}_{2}$ into the atmosphere (Carn et al. 2003). This eruption was unexpectedly captured by the Total Ozone Mapping Spectrometer (TOMS) which is used to detect ozone in the ultraviolet (UV) part of the spectrum (Krueger 1983; Krueger 
et al. 1995). The $\mathrm{SO}_{2}$ absorption feature was recognized and initiated the development of $\mathrm{SO}_{2}$ retrieval schemes for thermal infrared sensors and airborne sensors, such as the Thermal Infrared Multispectral Scanner (TIMS), Moderate Resolution Imaging Spectroradiometer (MODIS) and the Advanced Spaceborne Thermal Emission and Reflection Radiometer (ASTER), to quantify volcanic $\mathrm{SO}_{2}$. Such sensors and TOMS have provided volumes of data for volcanologists. For example, TOMS has viewed 194 eruptive events and 100 eruptions from 60 volcanoes over a period of 22 years (Carn et al. 2003).

The Ozone Monitoring Instrument (OMI) was launched in 2004 on the AURA platform and is the successor of the TOMS instrument, which was in operation from 1978 to 2005 (Yang et al. 2007). OMI plans to continue to add to the TOMS database, and expand on the number of volcanic plumes detectable by TOMS. This is due to having a lower $\mathrm{SO}_{2}$ detection limit. A lower detection limit is possible due to OMI having multiple wavelengths to measure $\mathrm{SO}_{2}$, reduced radiometric noise, and a smaller footprint (13 x 24 km) (Carn et al. 2003; Carn et al. 2008). The detection limits for OMI in comparison to TOMS for a plume velocity of $1 \mathrm{~m} / \mathrm{s}$ are $19 \mathrm{t} \mathrm{d}^{-1}$ and $1010 \mathrm{t} \mathrm{d}^{-1}$, respectively (Carn et al. 2003). Carn et al. (2008) explored the use of OMl to obtain daily measurements of volcanoes in the northern Andes in order to see if individual sources of $\mathrm{SO}_{2}$ can be identified, in order to establish trends and changes in daily $\mathrm{SO}_{2}$ emissions. This piece of research established that routine monitoring of volcanic $\mathrm{SO}_{2}$ for passively degassing volcanoes is possible for large high altitude emitters of $\mathrm{SO}_{2}$.

Unlike UV retrievals, infrared (IR) $\mathrm{SO}_{2}$ retrievals have to compete with water vapor absorption in the same wavelength regions as $\mathrm{SO}_{2}$ (Carn et al. 2003). This means that IR sensors are often less sensitive than those that operate in the UV (Kristiansen et al. 2010). Realmuto et al. (1994) developed a retrieval scheme in order to determine the column abundance of $\mathrm{SO}_{2} \mathrm{~g} \mathrm{~m}^{-2}$ in a plume 
using the IR sensor TIMS. $\mathrm{SO}_{2}$ retrievals using TIMS have been used to observe degassing volcanoes that produce a large $\mathrm{SO}_{2}$ emission rate $(<5000 \mathrm{t}$ $\mathrm{d}^{-1}$ ) such as Mt Etna, Sicily and Kilauea, Hawaii (Realmuto et al. 1994; Realmuto et al. 1997; Realmuto 2000). ASTER is a unique IR sensor in that it has a high spatial resolution of $90 \mathrm{~m}$ in the thermal IR (Table 1.1). Such a high spatial resolution allows for it to look at small scale passively degassing volcanoes. Realmuto et al. (1997) and Realmuto (2000) used this retrieval and TIMS data to simulate the response of ASTER in detecting $\mathrm{SO}_{2}$ plumes. The results obtained from these studies illustrated that ASTER is capable of detecting plumes from Kilauea and Etna with an $\mathrm{SO}_{2}$ column abundance of $10 \mathrm{~g}$ $\mathrm{m}^{-2}$ and $0.5 \mathrm{~g} \mathrm{~m}^{-2}$ respectively. Henney (2006) built on this research, investigating the detection limits of ASTER when sensing $\mathrm{SO}_{2}$. ASTER imagery of a low emitter of $\mathrm{SO}_{2}$ (Fuego, Guatemala) and a control volcano (Pacaya, Guatemala) were analyzed. Henney (2006) demonstrated that ASTER could easily detect $\mathrm{SO}_{2}$ emissions in excess of $1000 \mathrm{t} \mathrm{d}^{-1}$ at Pacaya volcano. It was uncertain if $\mathrm{SO}_{2}$ was being detected at Fuego volcano, with an average 20 year flux of $160 \mathrm{t} \mathrm{d}^{-1}$ (Andres et al.1993), which would indicate that ASTER was working close to its detection limit. A forward model was also used to simulate the response of ASTER and suggested that in a tropical atmosphere as little as $1 \mathrm{~g} \mathrm{~m}^{-2}$ could be detected (Realmuto 2000). Being potentially able to detect $\mathrm{SO}_{2}$ emissions with column amounts of $1 \mathrm{~g} \mathrm{~m}^{-2}$ is important in terms of the number of volcanoes that can be investigated (Realmuto 2000). It is not restricted to large emitters such as Etna and Kilauea. 
Table 1.1

ASTER subsystem characteristics. Source:www.asterweb.jpl.nasa.gov.

\begin{tabular}{|c|c|c|c|c|c|}
\hline Subsystem & Channels & $\begin{array}{l}\text { Spectral } \\
\text { Range }(\mu \mathrm{m})\end{array}$ & $\begin{array}{l}\text { Spatial } \\
\text { Resolution } \\
\text { (m) }\end{array}$ & $\begin{array}{l}\text { Swath } \\
\text { Width } \\
(\mathrm{km})\end{array}$ & $\begin{array}{l}\text { Cross Track } \\
\text { Pointing in } \\
\text { degrees } \\
\text { and km }\end{array}$ \\
\hline \multirow{4}{*}{$\begin{array}{l}\text { Visible } \\
\text { Near } \\
\text { Infrared }\end{array}$} & 1 & $0.52-0.60$ & \multirow[t]{4}{*}{15} & \multirow[t]{4}{*}{60} & \multirow{4}{*}{$\begin{array}{l}+24 \\
+318\end{array}$} \\
\hline & 2 & $0.63-0.69$ & & & \\
\hline & $3 \mathrm{~N}$ & $0.78-0.86$ & & & \\
\hline & $3 \mathrm{~B}$ & $0.78-0.86$ & & & \\
\hline \multirow{6}{*}{$\begin{array}{l}\text { Short Wave } \\
\text { Infrared }\end{array}$} & 4 & $1.60-1.70$ & \multirow[t]{6}{*}{30} & \multirow[t]{6}{*}{60} & \multirow{6}{*}{$\begin{array}{l}+8.55 \\
+116\end{array}$} \\
\hline & 5 & $2.145-2.185$ & & & \\
\hline & 6 & $2.185-2.225$ & & & \\
\hline & 7 & $2.235-2.285$ & & & \\
\hline & 8 & $2.295-2.365$ & & & \\
\hline & 9 & $2.360-2.430$ & & & \\
\hline \multirow{5}{*}{$\begin{array}{l}\text { Thermal } \\
\text { Infrared }\end{array}$} & 10 & $8.125-8.475$ & \multirow[t]{5}{*}{90} & \multirow[t]{5}{*}{60} & \multirow{5}{*}{$\begin{array}{l}+8.55 \\
+116\end{array}$} \\
\hline & 11 & $8.475-8.825$ & & & \\
\hline & 12 & $8.925-9.275$ & & & \\
\hline & 13 & $10.25-10.95$ & & & \\
\hline & 14 & $10.95-11.65$ & & & \\
\hline
\end{tabular}

Satellite validation is an important tool to determine how reliable and effective an instrument is at quantifying certain components of the atmosphere such as $\mathrm{SO}_{2}$ and ash. Few studies have been carried out to validate instruments at detecting products of volcanoes such as gas and ash and compare them to other techniques. ASTER has had sensitivity analysis carried out (Campion et al. 2010) and comparisons to COSPEC at Miyakejima volcano, Japan (Urai 2004) to validate this instrument and determine where it can be beneficial in detecting $\mathrm{SO}_{2}$ without under- or over- estimating emissions.

The sensitivity analysis by Campion et al. (2010) investigated how reliable ASTER was at detecting $\mathrm{SO}_{2}$ under varying conditions such as atmospheric humidity, surface altitude, ground temperature (thermal contrast between the plume and the ground), and the emissivity. The most sensitive parameter was found to be the temperature contrast between the ground and plume with $\mathrm{SO}_{2}$ being undetectable when the two have zero temperature contrast. Sensitivity 
increases with increasing thermal contrast. Water vapor is the primary gas that interferes with the detection of $\mathrm{SO}_{2}$ (at $8.5 \mu \mathrm{m} \mathrm{SO}$ absorption feature). Water vapor is abundant in the lower troposphere with $95 \%$ of its total residing in the lowest $4 \mathrm{~km}$ of the atmosphere (Campion et al. 2010). The majority of the plumes investigated in this study lie within the lower $4 \mathrm{~km}$ of the atmosphere and so water vapor may be an issue. However, sensitivity analysis by Campion et al. (2010), illustrates that humidity may not impact the retrieval of $\mathrm{SO}_{2}$ as much as previously thought. Even so, this factor does cause the surface altitude to have an effect on the retrieval when humidity is high.

Comparisons with ground based sensors have been carried out by Urai (2004) and to some extent Campion et al. (2010). Campion et al. (2010) compared COSPEC data to ASTER imagery collected at Miyakejima volcano, Japan. The COSPEC and ASTER measurements were in good agreement, but they were not considered as a validation of the ASTER $\mathrm{SO}_{2}$ retrieval because the two data sets were not collected simultaneously. The study by Urai (2004) contains data of a plume also from Miyakejima where 3 COSPEC transects were taken on the same day as an ASTER image was retrieved. There was a 1-2 hour difference between the COSPEC measurements and the time the ASTER image was acquired. The 3 COSPEC transects were plotted on the ASTER image and compared. It was found that 2 out of the 3 transects were in good agreement.

Few studies exist on the validation of $\mathrm{OMI}$ at detecting $\mathrm{SO}_{2}$ from volcanic plumes. There are three studies by Spinei et al. (2010), Carn et al. (2011) and Carn and Lopez (2011) where a validation of volcanic $\mathrm{SO}_{2}$ measurements from $\mathrm{OMI}$ has taken place by comparing the results to measurements acquired from ground based instruments. Spinei et al. (2010) validated $\mathrm{OMI} \mathrm{SO}_{2}$ retrievals from the volcanic cloud produced by Okmok, Alaska, on 12 July 2008, by comparing the results to the multifunction differential optical spectroscopy (MFDOAS). The MFDOAS took three measurements of the plume when it 
passed over Washington State over a period of three days. The results for these three MFDOAS measurements were in excellent agreement with the OMI measurements validating the reliability of the $\mathrm{OMI} \mathrm{SO}_{2}$ retrieval.

Carn et al. (2011) attempted to validate $\mathrm{OMI} \mathrm{SO}_{2}$ measurements to data collected during the Tropical Composition, Cloud and Climate Coupling ( $\mathrm{TC}^{4}$ ) mission where a NASA DC-8 research aircraft penetrated plumes from volcanoes in Ecuador and Colombia. Validation was not achieved for the OMI measurements at Tungurahua, Ecuador due to air traffic control restrictions. Only the distal portion of the plume could be measured and the $\mathrm{TC}^{4} \mathrm{SO}_{2}$ quantities were too low from this area to validate OMI for a single pixel. OMI temporal averages calculated over the time period of the $\mathrm{TC}^{4}$ mission $\left(10-17^{\text {th }}\right.$ July 2007) were compared to $\mathrm{TC}^{4}$ data on the $17^{\text {th }}$ July 2007 and were consistent with one another.

At Nevado del Huila, Colombia validation was difficult due to problems with the timing of the $\mathrm{OMI}$ image acquisition and the $\mathrm{TC}^{4}$ data. The $\mathrm{OMI}$ overpass occurred 3 hours after the $\mathrm{TC}^{4}$ data was collected. Significant changes in the plume would be expected on such a timescale and would not allow an accurate comparison of the two data sets. Cloud cover was also extensive in the OMI image which will impact the retrieved $\mathrm{SO}_{2}$. Other issues with the validation were that the DC-8 did not sample the whole vertical extent of the plume and the region of interest was viewed off nadir by OMI which produced large footprint sizes. These limitations made it difficult to validate the OMI data. Due to the DC8 not sampling the whole plume its data had to be interpolated to compare to OMI. The column abundance of $\mathrm{SO}_{2}$ retrieved for $\mathrm{OMI}$ was in the same order of magnitude as the $\mathrm{TC}^{4}$ data however, due to the limitations listed above (cloud cover, viewing angle, timing) this was not considered a true validation. 
Carn and Lopez (2011) attempted to validate the $\mathrm{OMI} \mathrm{SO} 2$ retrieval of the volcanic cloud produced from Sarychev Peak, Kurile Islands, using a mobile ground based ultraviolet spectrometer (FLYSPEC). It was found that there was a good agreement between five selected OMI pixels that the FLYSPEC traverse interested and the spatially averaged FLYSPEC $\mathrm{SO}_{2}$ data. Comparing the two data sets revealed some significant differences but this was attributed to the timing of the measurements, as the FLYSPEC collected data 40-80 minutes on either side of the $\mathrm{OMI}$ acquisition, and the spatial averaging of the $\mathrm{SO}_{2}$ over the OMI pixels. These problems relate to the fact that the volcanic cloud was constantly moving and it is spatially heterogeneous.

Several studies exist where more than one satellite sensor has been used to investigate an eruption (Schneider et al. 1999; Rose et al. 2003; Thomas et al. 2009). Few studies exist where the relationship between satellite sensors is compared. Thomas et al. (2009) directly compares three sensors (TOMS, OMI and MODIS) at an eruption from Sierra Negra volcano, Galapagos Islands. The three sensors in this study all have different advantages and limitations to one another such as sensitivity to $\mathrm{SO}_{2}$, spatial resolution, impact of competing species such as ash and water vapor and an established extensive data set. The study aimed to compare all there sensors pixel by pixel, but due to the time difference between image acquisitions this was not possible. Thomas et al. (2009) concluded that, (i) the $\mathrm{SO}_{2}$ tonnage for the three sensors was variable and dependent on plume altitude, (ii) $\mathrm{OMI}$ is more sensitive to $\mathrm{SO}_{2}$ than MODIS, (iii) Spatial comparisons were affected by altitude and geometry differences of the sensors as well as their associated errors, (iv) the total tonnage of $\mathrm{SO}_{2}$ for MODIS was greater than that of TOMS and OMI. This was expected to be a direct result of low level $\mathrm{SO}_{2}$ being retrieved or because of interference from other species resulting in an overestimation of $\mathrm{SO}_{2}$. 


\subsubsection{Previous Estimations of Global Volcanic $\mathrm{SO}_{2}$ Emissions in to the}

Atmosphere.

Previous estimates of global volcanic $\mathrm{SO}_{2}$ emissions in to the atmosphere have been quantified many times and have produced values between 1.5-50 million tons per year (Kellogg et al. 1972; Friend 1973; Stoiber and Jespen 1973; Cadle 1975; Le Guern 1982; Stoiber et al. 1987; Lambert et al. 1988; Andres and Kasgnoc 1998; Halmer et al. 2002). These estimates are unconstrained and have been produced using a variety of techniques (refer to Table 5.1 Chapter 5 for more details) such as melt inclusion data, isotopes (Lambert et al. 1988); lava density, gas content and volume of lava erupted (Kellogg et al. 1972; Friend 1973; Cadle 1975), and more recently ground and satellite based measurements (Andres and Kasgnoc 1998; Halmer et al. 2002).

Andres and Kasgnoc (1998) estimated that volcanoes contributed 13.4 million tons of $\mathrm{SO}_{2}$ into the atmosphere each year. These estimates were calculated using COSPEC data over a 25 -year period from 72 volcanoes. The volcanoes were split into two groups, continuously erupting volcanoes and sporadically erupting volcanoes. The continuously erupting volcanoes had average fluxes calculated for them to account for their natural variations in gas flux over time. It was presumed that the sporadic eruptions only lasted one day and therefore the maximum flux was used in this study. The total $\mathrm{SO}_{2}$ flux for these volcanoes is 9.66 million tons/yr. This was the minimum value calculated. This value does not account for those volcanoes that are not monitored or have not had measurements taken due to access problems. Andres and Kasgnoc (1998) use a power law to account for these unmeasured volcanoes that most likely account for continuously degassing volcanoes. The unmeasured volcanoes account for 1.85 million tons/yr of $\mathrm{SO}_{2}$ (Andres and Kasgnoc, 1998). It was suggested that estimates of 10 million tons/yr or less are too low while estimates above 20 million tons/yr are too high. 
Halmer et al. (2002) estimated a yearly global $\mathrm{SO}_{2}$ flux ranging from of 15 to 21 million tons/yr. They also calculated fluxes for the individual volcanic arcs and rifts. The two volcanic arcs that are of interest to this research are the South and Central American arcs. Halmer et al. (2002) estimated $\mathrm{SO}_{2}$ fluxes of 2.1-3.6 $\mathrm{Tg} / \mathrm{yr}$ and 0.6-0.8 Tg/yr for the South and Central American arcs respectively. These estimates are based on TOMS and COSPEC measurements, and the annual frequency of the assigned eruption activity by the volcanic explosivity index (VEI). Where no COSPEC and TOMS data are available (prior to 1979) the volcanic sulfur dioxide index (VSI) is used to infer an $\mathrm{SO}_{2}$ flux from historical eruptions back to 1900. The VSI was developed using the average sulfur emissions from a volcano and the VEI of their eruptions (Schnetzler et al. 1997). Halmer et al. (2002) modified the VSI by a factor of two due to the fact that the original values produced by the VSI were underestimating the average quantity of $\mathrm{SO}_{2}$ degassed form volcanoes. The new VSI is multiplied by the number of eruptions in a year to produce the annual global volcanic $\mathrm{SO}_{2}$ emissions from explosive eruptions for a given year. The calculated flux represents only a rough estimate because the majority of the gas measurements were assumed and predicted by the VEI and the VSI. Using this method only accounts for explosive eruptions not the average emissions during passive quiescent periods of activity. Additionally, COSPEC measurements are typically taken during eruptive episodes at volcanoes and so measurements are not continuous and or a true reflection of average passively degassing emissions from the volcano in question.

Volcanoes in the Central American arc are generally better studied and more recent estimates of total $\mathrm{SO}_{2}$ flux have been made. Mather et al. (2006) carried out field work at several volcanoes in Nicaragua and combined those results with recent work detailing fluxes from Guatemala, by Rodriguez et al. (2004), Costa Rica by Zimmer et al. (2004), and data from studies prior to 1997 to calculate an average arc flux of $3570 \mathrm{t} \mathrm{d}^{-1}$. Excluding the data prior to 1997 and 
just using more recent measurements obtained at volcanoes in the arc, a flux of $4320+/-1500 \mathrm{t} \mathrm{d}^{-1}$ was calculated. This flux contributes $8-16 \%$ of the estimated annual global volcanic $\mathrm{SO}_{2}$ flux to the troposphere based on the time averaged global volcanic emissions of 26,000 $-55,000 \mathrm{t} \mathrm{d}^{-1}$ (Stoiber et al. 1987; Andres and Kasgnoc 1998; Halmer et al. 2002). Mather et al. (2006) acknowledge it is uncertain that all the volcanoes along the Central American arc have been accounted for.

\subsubsection{Geologic Setting and Target Volcanoes}

The Central and South American volcanic arcs are the focus of this dissertation. Both arcs are home to approximately 230 active volcanoes of which around many are producing detectable amounts of $\mathrm{SO}_{2}$ (Global Volcanism Program, Siebert and Simkin 2002-). The volcanoes throughout the two arcs exhibit a range of styles of activity and magma compositions. For example, Masaya volcano, Nicaragua is basaltic in composition and is constantly degassing, while Satiaguito volcano, Guatemala is dacitic in composition and activity consists of continuous small to moderate explosions. The Central American arc runs for around $1500 \mathrm{~km}$ from the Mexican-Guatemalan border in the north-west to the Panamanian Colombian border in the south-east. The arc is a direct result of the subduction of the of the Cocos plate beneath the Caribbean plate. Along this arc there are changes in plate thickness, subduction angle, and convergence rate. This includes the fastest convergence rate $(90 \mathrm{~mm} / \mathrm{yr})$ and shallowest dip of the subducting crust is in Costa Rica. The slowest convergence rate $(70 \mathrm{~mm} / \mathrm{yr})$ is in Guatemala and the steepest subduction angle along the arc is located beneath Nicaragua (Protti et al. 1995; DeMets et al. 1990). The South American volcanic arc runs from Colombia in the north to Argentina and Chile in the south and is a product of the Nazca plate subducting underneath the South American plate. The South American arc can be subdivided in to four distinct zones of volcanic activity: the Northern Volcanic Zone runs from Columbia to Ecuador (from Nevado del Ruiz to Sangay), the Central 
Volcanic Zone runs from Peru to northern Chile (including volcanoes such as Ubinas and Lascar), the Southern Volcanic Zone covers Central Chile (including Villarrica and Chaiten), and the Austral Volcanic Zone runs from southern Chile to Patagonia. In between each of the zones there are no active volcanoes. This is a direct result of the subduction angle not being steep enough to allow melting of the crust to occur producing magma. Table 5.2 (Chapter 5 ) provides a brief description of many of the volcanoes in Central and South America that are investigated.

Two chapters of this dissertation focus specifically on Lascar volcano which is located in Northern Chile in the Central Volcanic Zone. Lascar is one of Chile's most active volcanoes with persistent degassing punctuated by major and minor explosions. Lascar is thought to be less than 50 ka old (Matthews et al. 1997 ) with historical records of activity dating back to 1848 . Lascar reactivated in 1984. Since 1984 few studies have investigated Lascar and those that do predominantly focus on thermal features through the use of remote sensing techniques (Francis and Rothery 1987; Rothery et al. 1987; Glaze et al. 1989 a; b; Oppenheimer et al. 1993; Wooster 2001; Murphy et al. 2011). A thermal anomaly was imaged at Lascar using the Landsat Thematic Mapper in 1984. Francis and Rothery 1987 assumed that this anomaly was a lava lake and not a dome with the presence of strong fumarolic degassing due to the composition of the volcano being basaltic andesite. If gas data had been collected, a lava lake might not have been assumed and alternative scenarios, such as a lava dome with fumaroles, would have been considered.

Occasional gas measurement have been collected at Lascar (Mather et al. 2006; Rodriguez 2007), but no study has looked at gas emissions and how they have changed over time in relation to activity seen at Lascar. Lascar's remote location is responsible for the few studies carried out; however, with sensors such as ASTER it is becoming easier to detect $\mathrm{SO}_{2}$ and other thermal features 
to monitor potential activity. An investigation in to the petrology and geochemistry of the volcanic products of Lascar indicated that the magma chamber was continuously fractionally crystalizing (Matthews et al. 1994b). Injections of newer magma of a basaltic andesitic composition were found to be introduced into the magma chamber periodically and mixed with the more evolved magma. Matthews et al. (1994b) suggest that the strong $\mathrm{SO}_{2}$ emissions from Lascar are a result of the oxidation and quenching of sulfide rich mafic magma when it mixes with the older more evolved magma in the chamber. 


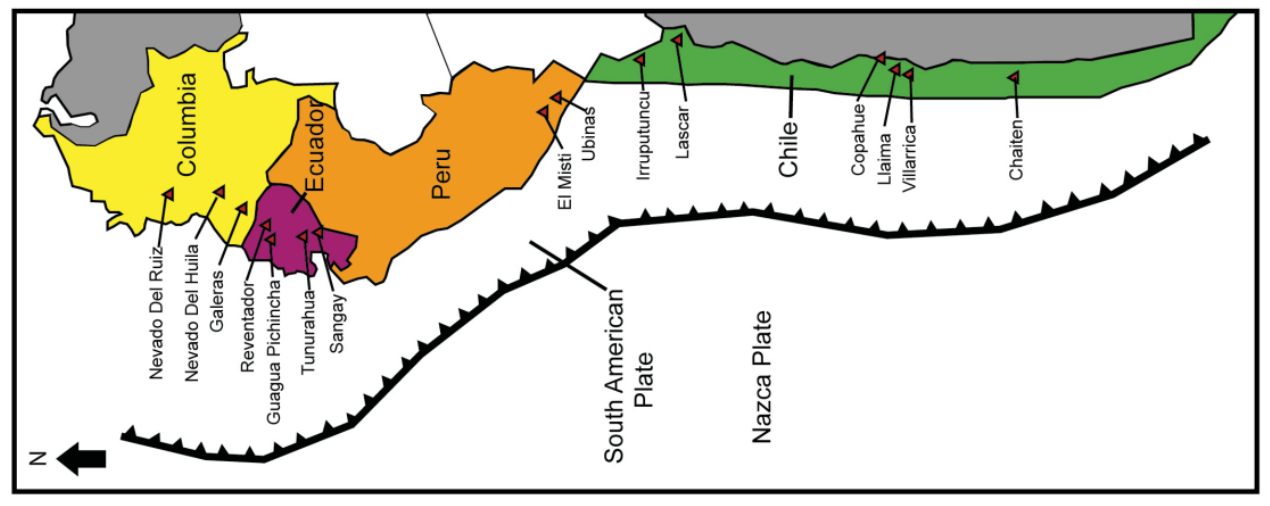

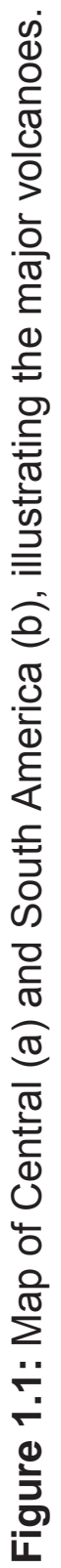




\subsection{Methodology-Sensor Descriptions and Algorithms}

\subsubsection{ASTER instrument description}

The Advanced Spacebourne Thermal Emission and Reflection Radiometer (ASTER) is one of five sensors on board NASA's EOS Terra Platform that was launched 18 December 1999. The Terra platform has a near polar orbit, crossing the equator at 10:30 UTC each day at an altitude of $705 \mathrm{~km}$ above the Earth's surface (Ramsey and Dehn 2004). ASTER has a broad spectral range with 14 channels across the visible to thermal infrared regions. ASTER is made up of three subsystems, each of which looks at a particular region of the spectrum (Yamaguchi et al. 1998) (Table 1.1). The near visible infrared (NVIR) subsystem is made up of 3 channels with a spatial resolution of 15 meters and a second telescope to allow backward looking images to be obtained. The shortwave infrared (SWIR) subsystem has 6 channels and a spatial resolution of 30 meters. The thermal infrared (TIR) subsystem has 5 channels at a spatial resolution of 90 meters. With ASTER being pointable up to $22.5^{\circ}$ off nadir and an $8 \%$ duty cycle, areas of interest can be captured every 5 days and possibly more in high latitude areas (Abrams 2000). However, the normal temporal resolution of ASTER is an image every 16 days (Ramsey and Dehn 2004). With the routine night time acquisition of SWIR and TIR data for high temperature targets, more data can be collected at active volcanoes, especially those in high latitudes during the winter. With pre-scheduled flights mitigation can occur against the saturation of poor images over highly reflective, noisy, low signal targets. Additionally, with a backward pointing telescope in the VNIR as well as a nadir viewing telescope in the same spectral range (band $3 \mathrm{~B}$ and $3 \mathrm{~N}$ respectively) a stereoscopic pair of images can be obtained to produce a digital elevation model (DEM) with a resolution of 15m (Ramsey and Dehn 2004).

ASTER is a useful tool for investigating volcanic activity. However, with a low temporal resolution, ASTER is not ideal for near real time monitoring of volcanoes. Yet, with such high spatial resolution and spectral range ASTER can 
produce a time series of detailed images capturing a volcano's complete eruptive activity cycle, given that the sensor can be (and is regularly) targeted at new and continuing activity.

Compiling information on a volcano's complete eruptive cycle can provide insight into how it behaves. Precursory signals, such as a deviation from baseline levels, can be established such as changes in $\mathrm{SO}_{2}$ emissions or fumarole temperatures. For example, at Mt Etna a decrease in the baseline $\mathrm{SO}_{2}$ emission rates has been recognized prior to an eruption (Caltabiano et al. 1994). The thermal energy output of lava flows, pyroclastic flows, and hotspots can be determined more accurately with a higher spatial resolution in the TIR. The detection of precursor eruption hotspots that have a low thermal contrast compared to background pixels can be distinguished. The effusion rate, cooling rate, and the speed at which a lava flow is moving can be determined using a time series of images (Pieri and Abrams 2004). Higher resolution in the TIR (90 $\mathrm{m})$ facilitates the detection of $\mathrm{SO}_{2}$ plumes that are too dispersed or contain quantities that are too small for sensors such as TOMS and MODIS to detect (Realmuto 2000).

\subsubsection{ASTER TIR retrieval Algorithm (MAP SO2)}

$\mathrm{SO}_{2}$ in the atmosphere reduces atmospheric transmission between $8 \mu \mathrm{m}$ and $9.5 \mu \mathrm{m}$ in the thermal infrared. Channels 10,11 and 12 of ASTER cover this part of the spectrum (Table 1.1) and can be utilized for $\mathrm{SO}_{2}$ retrieval. Figure 1.2 (a) illustrates the ASTER response functions and (b) $\mathrm{SO}_{2} 8.6 \mu \mathrm{m}$ absorption feature.The retrieval of $\mathrm{SO}_{2}$ is based on the at-sensor radiance expression developed by Realmuto et al. (1994):

$$
L_{s}=\left\{\varepsilon_{g} B\left(T_{g}\right)+\left(1-\varepsilon_{g}\right) L_{d}\right\} t_{a}+L_{u}
$$


where $\varepsilon_{g}$ is the ground emissivity, B, the Planck function, $T_{g}$, the temperature of the ground, $t_{a}$, the transmission of the atmosphere, $L_{d}$, the downwelling radiance and $L_{u}$ the upwelling radiance. The IDL code that uses this expression, MAP_SO2, is based on the MODTRAN radiative transfer code (Berk et al. 1989) and can be used to determine the column abundance of $\mathrm{SO}_{2}$ using the TIR data of ASTER (Realmuto and Worden 2000). The radiative transfer code contains spectral band models of 12 atmospheric gas molecules, including $\mathrm{SO}_{2}$. These band models are used along with a user-defined atmosphere, from which upwelling and downwelling radiances are derived, and the spectral emissivity of the ground to calculate the radiance received at the sensor. The spectral emissivity of the ground is determined using spectral normalization (Realmuto 1994; Watson et al. 2004) where one value of the spectral emissivity (the maximum) is allowed to float. The upwelling and downwelling radiances and the transmission of a user-defined atmosphere are produced using the radiative transfer code, based upon acquired atmospheric radiosonde data pertinent to the image location (Watson et al. 2004).

As none of the parameters mentioned above can be easily determined by the at-sensor radiance spectra, the retrieval of $\mathrm{SO}_{2}$ is a complex inverse problem (Realmuto et al. 1997; Realmuto 2000). Additional information is loaded into MAP_SO2, such as plume altitude and thickness to make the inverse problem better constrained. With this additional information only the $\mathrm{SO}_{2}$ column abundance, emissivity and the ground temperature need to be considered. To determine the $\mathrm{SO}_{2}$ column abundance the 'true' ground temperature and emissivity have to be calculated using the at-sensor radiance expression (Eq. (1)) in ASTER channels 13 and 14 as they lie outside the $\mathrm{SO}_{2}$ absorption feature. The 'apparent' ground temperature is determined by solving the at sensor radiance expression (Eq. (1)) using channels lying within the $\mathrm{SO}_{2}$ absorption feature (channels 10,11 and 12). It is the difference between the true and apparent ground temperatures that allow the $\mathrm{SO}_{2}$ loading to be calculated, 
as the temperature difference is a linear function of the $\mathrm{SO}_{2}$ burden (Realmuto 1990; Watson et al. 2004). The result is an $\mathrm{SO}_{2}$ map of the dimensions of the original image with the units of $\mathrm{g} \mathrm{SO}_{2}$ per $\mathrm{m}^{-2}$ (i.e. column integrated $\mathrm{SO}_{2}$ burden).

The emission rate of $\mathrm{SO}_{2}$ emitted from a volcano is calculated using the width of the plume, wind speed and the average $\mathrm{SO}_{2} \mathrm{~g} \mathrm{~m}^{-2}$. The width is derived from the number of pixels across the plume multiplied by the spatial resolution of these pixels (90 $\mathrm{m}$ in the TIR at nadir). The $\mathrm{SO}_{2} \mathrm{~g} \mathrm{~m}^{-2}$ is calculated by integrating all the $\mathrm{SO}_{2} \mathrm{~g} \mathrm{~m}^{-2}$ values for each pixel across the plume width:

$\mathrm{SO}_{2}$ emission rate $\left(\mathrm{g} \mathrm{s}^{-1}\right)=$

width of plume $(\mathrm{m})^{*}$ wind speed $\left(\mathrm{m} \mathrm{s}^{-1}\right)^{*}$ integrated $\mathrm{SO}_{2}\left(\mathrm{~g} \mathrm{~m}^{-2}\right)$

The resulting flux is in $\mathrm{g} \mathrm{s}^{-1}$. 


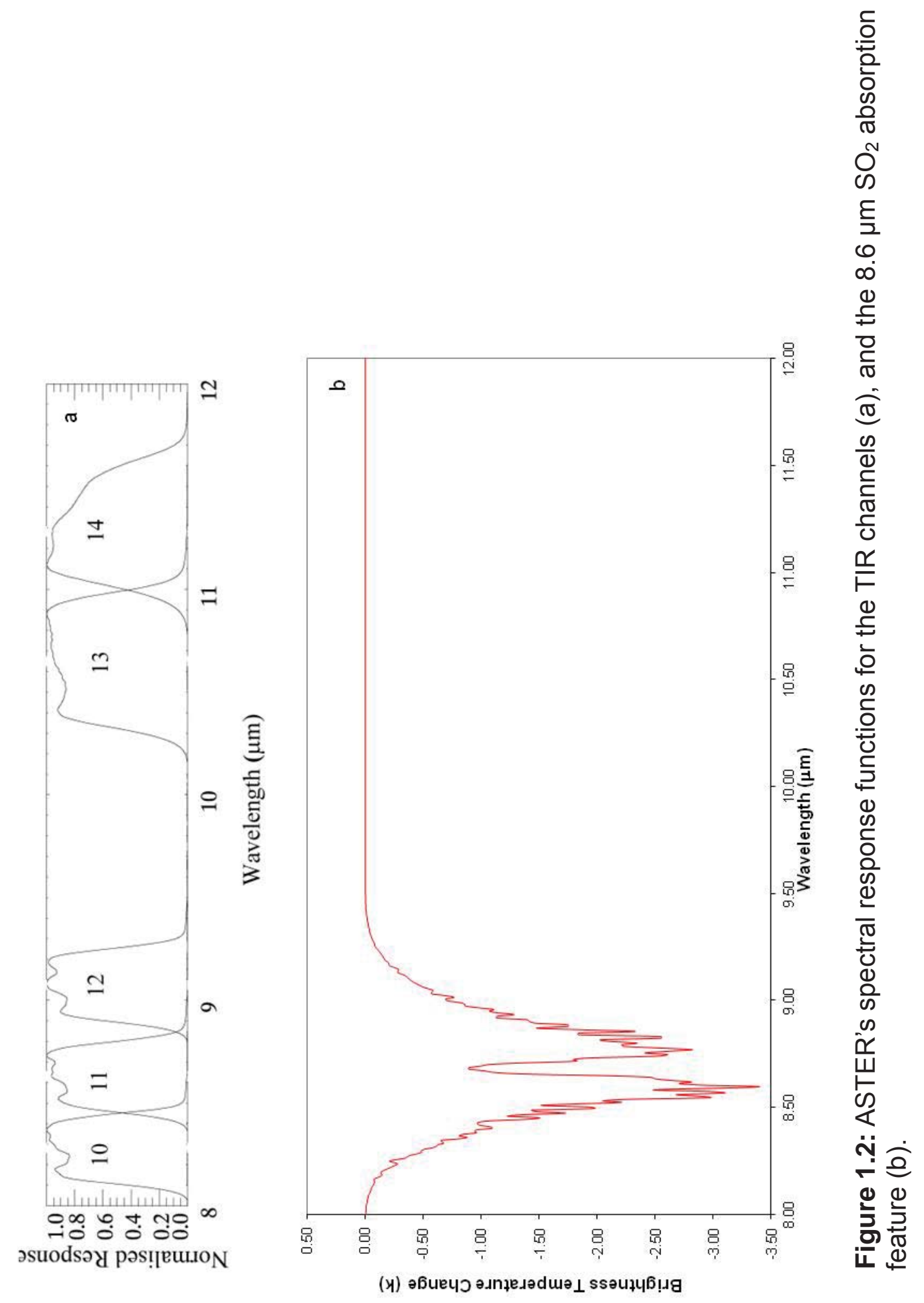




\subsubsection{ASTER Errors}

The accuracy of the MAP_SO $\mathrm{SO}_{2}$ procedure is dependent on the precision of radiance measurements and any assumptions made in describing the transfer of radiation from the ground to the sensor (Realmuto et al. 1997). Errors in the retrieval are associated with the instrument calibration and sensitivity, and the accuracy of the MAP_SO $\mathrm{SO}_{2}$ procedure. The instrument sensitivity must be taken in to consideration when looking at the errors as it relates to the accuracy and precision of the instrument. The sensitivity of the instrument is known as the Noise equivalent change in temperature $(\mathrm{NE} \Delta \mathrm{T})$. The $\mathrm{NE} \Delta \mathrm{T}$ is the smallest change in temperature that would be needed to equal the noise of the sensor in question. The NE $\Delta$ T for the ASTER TIR bands is $0.12 \mathrm{~K}$.

Other important parameters that are taken into account in the retrieval process are the plume altitude and thickness and the local atmospheric conditions. Any uncertainties in our knowledge of these factors will generate additional errors that are propagated throughout the algorithm. The plume altitude is a significant factor in that it defines the temperature contrast between the ground and the plume (Realmuto 1994; 2000; Campion et al. 2010). Campion et al. (2010) carried out a sensitivity analysis investigating the effects of ground temperature, plume temperature, and altitude. It was found that $\mathrm{SO}_{2}$ is undetectable when the ground and plume temperature are identical. The algorithm is based on the thermal contrast between the ground and the plume. If there is zero thermal contrast the plume cannot be distinguished. As the temperature contrast increased so did the sensitivity to $\mathrm{SO}_{2}$. The MAP_SO2 model assumes that volcanic plumes are laminar with a uniform thickness and that they are in equilibrium with the local atmosphere (Realmuto et al. 1994; 1997; Realmuto 2000). Many plumes contain discrete puffs emitted from the volcano. Often these differences in thickness (puffs) are mapped as differences in $\mathrm{SO}_{2}$ column abundance (Reamuto et al. 1997). Other issues related to plume geometry relate to the fact that often there is no information on the height or thickness of 
the plume in question due to no visual observations. Often these parameters have to be estimated. An error of $\pm 10 \%$ is related to inaccurate plume geometry. To minimize this error Realmuto (2000), suggests that if no information or visual observations are available about the height of the plume it should be assumed to be at the vent altitude.

Other means of determining the altitude and thickness of the plume include shadows produced by the plume along with the time of image acquisition and solar azimuth (Glaze et al. 1989; Holasek and Self 1995; Realmuto et al. 1994; Realmuto 2000). Urai (2000) used a stereoscopic pair of nadir and backward pointing images along with their viewing angles and the time difference between them to calculate the plume altitude. Stereoscopic pairs as previously mentioned (section 1.3.1) can be used to generate DEMs that can, with some manipulation, be used to model volcanic plumes and infer their altitude.

The atmospheric conditions are determined using atmospheric radiosonde profiles from the closest weather station to the volcano in question. However, these stations are often quite a distance away from the volcano and are not accurate or a true reflection of the local climate. The wind speed will not be accurate and the percentage of water in the atmosphere may be quite different. Both these parameters are significant in calculating the $\mathrm{SO}_{2}$ load and determining detection limits. Many sensitivity analyses have been carried out to assess how much of an error there is as a result of inaccurate climate data. Results from Henney (2006) suggest the retrieval is more robust than expected with MAP_SO2 providing consistent results with varying radiosonde profiles.

Realmuto and Worden (2000) carried out work, in order to compare the $\mathrm{SO}_{2}$ flux calculated at the Pu' 'O'O vent of Kilauea, Hawaii using local atmospheric data and distant atmospheric data. The results indicated that there was a $\sim 25 \%$ difference between the $\mathrm{SO}_{2}$ column abundances calculated for local and distant 
atmospheric data. Such a study indicated the importance of accurate atmospheric data and how sensitive the $\mathrm{SO}_{2}$ retrieval is, especially to water vapor. The study pointed out that this level of sensitivity to atmospheric conditions is analogous to $\mathrm{SO}_{2}$ retrievals from COSPEC (Millan 1980; Stoiber et al. 1983) and TOMS (Krueger et al. 1995).

Studies that have been conducted using thermal infrared airborne sensors such as TIMS (and simulated ASTER and MODIS data) to retrieve the $\mathrm{SO}_{2}$ column abundance have had a minimum error budget that range between 15\% and 25\% (Realmuto et al. 1994; 1997; Realmuto 2000; Realmuto and Worden 2000; Campion et al. 2010). Campion et al. (2010) estimated a minimum error of $25 \%$ but only when the thermal contrast between the plume and the ground was sufficient $\left(>10^{\circ} \mathrm{C}\right)$. A maximum error of $31 \%$ was calculated by Henney (2006) for ASTER detecting $\mathrm{SO}_{2}$ at Feugo and Pacaya volcanoes in Guatemala.

\subsubsection{OMI Instrument Description}

The Ozone Monitoring Instrument (OMI) was launched in July 2004 on board NASA's Aura platform. OMI is a hyper-spectral instrument that operates in the Visible and Ultraviolet (UV) part of the spectrum. With a $2600 \mathrm{~km}$ swath width and spatial resolution of 13 by $24 \mathrm{~km}$, OMl is capable of daily global coverage. $\mathrm{OMI}$ is used to detect many trace gases that are pertinent to air quality and climate control. Gases detected include $\mathrm{O}_{3}, \mathrm{HCHO}, \mathrm{BrO}$ and $\mathrm{SO}_{2} . \mathrm{O}_{3}$ and $\mathrm{SO}_{2}$ are detected using the UV-2 channel which has a spectral resolution of $0.4 \mathrm{~nm}$. This channel covers a large portion of the $\mathrm{SO}_{2}$ absorption feature (Carn et al. 2008). OMI is considered a replacement for the Total Ozone Mapping Spectrometer (TOMS). TOMS operated from 1979 and provided an extensive record of many large volcanic eruptions (Carn 2004). OMI has a higher sensitivity to $\mathrm{SO}_{2}$ in comparison to TOMS. OMI can detect $\sim 19 \mathrm{t} \mathrm{d}^{-1}$ for a plume travelling at $1 \mathrm{~m} \mathrm{~s}^{-1}$, whereas TOMS can only detect as little as $\sim 1010 \mathrm{t} \mathrm{d}^{-1}$ (Carn 2008). These values are general guidelines. 


\subsubsection{OMI UV retrieval Algorithm}

For each $\mathrm{OMI}$ scene four estimates of the column density of $\mathrm{SO}_{2}$ are calculated by making assumptions about the vertical distribution of $\mathrm{SO}_{2}$. The four estimations are made for the planetary boundary layer $\mathrm{SO}_{2}$ column (PBL) at $0.9 \mathrm{~km}$, lower tropospheric $\mathrm{SO}_{2}$ column $(\mathrm{TRL})$ at $2.5 \mathrm{~km}$, middle tropospheric $\mathrm{SO}_{2}$ column (TRM) at $7.5 \mathrm{~km}$ and the upper stratospheric $\mathrm{SO}_{2}(\mathrm{STL})$ column at $17.5 \mathrm{~km}$. Two algorithms are used to calculated $\mathrm{SO}_{2}$ column data, the Band Residual Difference (BRD) algorithm (Krotkov et al. 2006) and the Linear Fit (LF) algorithm (Yang et al. 2007). The BRD algorithm is used to calculate the $\mathrm{PBL} \mathrm{SO}_{2}$ column while the LF algorithm is used to calculate $\mathrm{SO}_{2}$ columns for the LRL, TRM, and STL (http://so2.gsfc.nasa.gov).

The BRD algorithm uses four wavelength bands in the UV-2 between 310-365 $\mu \mathrm{m}$. In order to maximize the detection sensitivity to $\mathrm{SO}_{2}$ to small $\mathrm{SO}_{2}$ column quantities, the bands are centered over the maxima and minima of the $\mathrm{SO}_{2}$ absorption feature. This takes advantage of the differential absorption between the three pairs of bands maximizing the detection efficiency (Yang et al. 2007). However, the downside of maximizing the detection sensitivity to lower $\mathrm{SO}_{2}$ column amounts is that the BRD algorithm becomes inadequate for detecting large quantities of $\mathrm{SO}_{2}$ injected into the atmosphere when the band residual differences of the three pairs show a nonlinear response to $\mathrm{SO}_{2}$ increments (Yang et al. 2007). For more detail about the BRD algorithm refer to Krotkov et al. (2006).

The LF algorithm is an improvement on the BRD algorithm specifically when retrieving data for high $\mathrm{SO}_{2}$ column amounts (Yang et al. 2007). The LF algorithm is based on a modified version of the TOMS total ozone algorithm, OMTO3, and utilizes the differential absorptions of ozone and $\mathrm{SO}_{2}$. Backscattered UV radiances (BUV) from ten wavelength bands, including the four bands used in the BRD algorithm, are used in a forward model. The 
forward model calculates radiance for the top of the atmosphere (TOA) for each band and compares them to the actual measured TOA radiances.

The BUV radiance for each band can be expressed by the following relationship:

$$
\log _{10} I_{m}=\log _{10} I(\Omega, \equiv, R)+\varepsilon
$$

where modeled radiance is $I$, ozone is $\Omega, S_{2}$ is $\Xi$, and surface reflectivity is $R$. For the OMI retrieval equation (3) is expressed as:

$$
N_{m}=N\left((\Omega, \equiv, R)+\varepsilon_{T}\right.
$$

$\mathrm{N}$ is a dimensionless quantity and combined with $\varepsilon_{T}$, is a combination of measurement and model errors for each band. $\Omega, \Xi$, and R, are adjusted until the radiances calculated from the forward model are equivalent to the measured radiances at the 10 bands, and a $\mathrm{SO}_{2}$ column abundance can be determined.

\subsubsection{OMI Errors}

UV sensors such as OMI and TOMS have errors that typically increase with solar zenith angle, ozone column, $\mathrm{SO}_{2}$ column amounts and in the presence of cloud and large quantities of aerosols (Krotkov et al. 2006). Cloud cover can impact the retrieval of $\mathrm{SO}_{2}$ by shielding $\mathrm{SO}_{2}$ at low altitudes or enhance the $\mathrm{SO}_{2}$ signal resulting in an overestimation of the column $\mathrm{SO}_{2}$ amount. The effects of aerosols on UV measurements usually results in an overestimation of $\mathrm{SO}_{2}$. Ash, haze and older emissions left in the atmosphere can also cause errors. However, the retrieval algorithm can account for such problems. The TOMRAD forward model accounts for the existence of aerosols. The error associated with TOMS retrieving $\mathrm{SO}_{2}$ from ash laden plumes was 30\% (Krueger et al. 1995; 
Krotkov et al. 2006; Carn et al. 2007; Carn et al. 2008). It is expected that the total error associated with OMI will be less than that of TOMS (Carn et al. 2008). One of the major problems with the $\mathrm{OMI} \mathrm{SO}_{2}$ retrieval is the non-linear effect. The Linear Fit algorithm can calculate $\mathrm{SO}_{2}$ efficiently up to $\sim 100 \mathrm{DU}$. However, when concentrations reach above 400 DU the algorithm starts to seriously underestimate the $\mathrm{SO}_{2}$ due to the non-linear effect (Krotkov et al. 2006; Yang et al. 2007; Thomas et al. 2009). This may cause an error of up to $70 \%$. 


\subsection{Summary of chapters}

This dissertation primarily investigates the use of remote sensing techniques to quantify $\mathrm{SO}_{2}$ from passively degassing volcanoes.

The first research chapter (Chapter 2) is a sensor validation and focuses on the ability of the Advanced Thermal Emission and Reflection Radiometer (ASTER) to detect $\mathrm{SO}_{2}$ in comparison to the Mini UV Spectrometer (MUSe). Satellite validation is of key importance especially since many volcanoes are in remote places where satellite based sensors may be the only observations made. Each sensor needs to be comparable to other sensors, and their ability and associated errors known. As previously mentioned, few studies have been carried out validating ASTER with ground based techniques. Studies have investigated errors and detection limits have been established using forward models and testing simulated data (Realmuto 1994; 1997; 2000; Henney 2006; Campion et al. 2010).

Chapter 3 investigates the use of image enhancing techniques to identify volcanic plumes from ASTER imagery. Often a volcanic plume is not visible in the raw data by the naked eye and so image enhancement techniques are used to highlight the plume. The two image enhancements used in this dissertation are a principal components analysis and decorrelation stretch. Little work has been previously carried out using these techniques to see to what extent they are capable of highlighting plumes from images and indicating what their compositions are. A variety of plumes are investigated under various conditions including those from explosive eruptions, passive emissions, at different altitudes, of varying $\mathrm{SO}_{2}$ concentrations and cloud cover.

In chapter 4 ASTER and OMI are utilized at Lascar volcano to quantify $\mathrm{SO}_{2}$ emissions. Lascar volcano, Chile, is remote and measurements are few and far between. Activity here has been well monitored by satellite based remote 
sensing in the past (1984-1996); however, recent activity over the last 5-10 years is not well understood. Cyclic behavior has been evident at Lascar; however, it is unknown if this pattern of activity is continuing. Using ASTER and OMI, Lascar's $\mathrm{SO}_{2}$ emissions will be quantified and temperatures of the crater determined and related to eruptive behavior.

Chapter 5 involves the application of ASTER and OMI to make an estimation of the contribution of $\mathrm{SO}_{2}$ into the troposphere from volcanoes in the Central and South American volcanic arcs. Both these sensors have a high precision and are more sensitive to detecting $\mathrm{SO}_{2}$ compared to TOMS or MODIS, allowing for a more accurate estimate of volcanic $\mathrm{SO}_{2}$ than previous attempts. This is important not only to determine baseline fluxes at volcanoes, for monitoring and prediction purposes, but also for climatic models. With the large quantity of satellite imagery processed for this research, some assumptions about ASTER and $\mathrm{OMI}$, and the detection limits of both instruments explored.

Chapter 6 summarizes and concludes the work carried out in this dissertation. It also offers recommendations for future work. 


\section{Chapter 2}

\section{A Comparison of $\mathrm{SO}_{2}$ Retrieval Techniques using Mini UV Spectrometers and ASTER Imagery at Lascar Volcano, Chile. ${ }^{1}$}

\footnotetext{
${ }^{1}$ Published in Bulletin of Volcanology (2012) 74 (2): 589-594 Copyright permission for Chapter 2
} 


\subsection{Introduction}

Measuring sulfur dioxide is an important part of the suite of geophysical techniques used to monitor activity at volcanoes, as changes in $\mathrm{SO}_{2}$ provide insight into what may be happening in the shallow conduit system (e.g., Edmonds et al. 2003). More specifically, monitoring $\mathrm{SO}_{2}$ at passively degassing volcanoes over long periods of time can provide baseline values, with deviations from the norm useful for predicting and mitigating against impending changes in eruptive activity. Passive production of $\mathrm{SO}_{2}$ can be detected using several ground-based instruments (Williams-Jones et al. 2008), such as miniUV spectrometers employing either differential optical absorption spectroscopy (DOAS) (e.g., Galle et al. 2002), correlation spectroscopy (e.g., Stoiber et al. 1983), or UV camera imagery (e.g., Mori and Burton 2006; Bluth et al. 2007; Dalton et al. 2009). Satellite imagery, such as the Ozone Monitoring Instrument (OMI) (e.g., Carn et al. 2008), the Moderate Resolution Imaging Spectroradiometer (MODIS) (e.g., Watson et al. 2004) and the Advanced Thermal Emission and Reflection Radiometer (ASTER) (e.g., Urai. 2004) can also be used to map larger non-explosive emissions of $\mathrm{SO}_{2}$. The ASTER sensor has 14 bands across the visible-near infrared (VNIR), shortwave infrared (SWIR) and thermal infrared (TIR). Five of these bands cover the TIR region of the spectrum and can be used to exploit the $8.6 \mu \mathrm{m} \mathrm{SO} \mathrm{SO}_{2}$ absorption feature (Realmuto. 2000; Watson et al. 2004). With a spatial resolution of $90 \mathrm{~m}$ in the TIR, ASTER is an especially useful instrument for detecting low $\mathrm{SO}_{2}$ fluxes at most passively degassing volcanoes. How well the ASTER retrieval compares to other techniques, such as the MUSe, is important in assessing its ability to quantify $\mathrm{SO}_{2}$, especially where an ASTER image may provide the only available emission-rate data at a volcano because of, for example, political instability or remoteness.

Lascar volcano, Chile, is a prodigious emitter of gas and typically has a visible plume above its crater (Gardeweg et al. 1994; Matthews et al. 1997). It is a 
good target for validation due to its high altitude (ca. $5500 \mathrm{~m}$ asl) and its being a consistently strong emitter of $\mathrm{SO}_{2}$. On $7^{\text {th }}$ December 2004 MUSe measurements were performed at Lascar volcano to investigate the volcano's plume. On the same day an ASTER overpass was scheduled and an image of Lascar and its plume was acquired at 11:47:18 local time. The MUSe measurements were taken continuously before, during, and after the ASTER pass. Here we will compare the fluxes calculated based on the ASTER image and based on the MUSe data. Comparison of ASTER with MUSe results reveals how well correlated the methods are, while also highlighting significant errors that need to be addressed. 


\subsection{Methodology}

\subsubsection{UV spectroscopy}

$\mathrm{SO}_{2}$ fluxes were calculated using mini-UV spectrometers (Edmonds et al. 2003), based on the principle of differential optical absorption spectroscopy (Platt. 1994; Galle et al. 2002). These methods have been recently detailed for this specific mini-UV spectrometer (MUSe) system (Rodríguez et al. 2008) and are only briefly described here. Measurements were acquired using standard techniques identical to the standard correlation spectrometer (COSPEC) rationale (Stoiber et al. 1983; Williams-Jones et al. 2008). For this particular experiment, two spectrometer systems were used simultaneously in stationary scanning mode (Edmonds et al. 2003). Errors associated with this technique are significant (McGonigle et al. 2003; Salerno et al. 2009), but our knowledge of them has improved. Each scan of the plume took about 120 seconds, and provided 15 scans, from each MUSe instrument, over a 30 minute period straddling the ASTER overpass at 11:47:18 local time. Each scan yielded approximately 60 spectra acquired at an integration (exposure) time of 1 second.

The raw data were then processed to remove instrument noise (dark current) and divided by the normalized clear sky spectrum (acquired outside of the volcanic plume) to isolate atmospheric scattering effects due to non-volcanic sources. The negative logarithm is calculated for each spectrum to produce a smooth spectrum that is a result of absorption due to volcanic sources. A laboratory spectrum was fitted to the absorbance spectra to retrieve the line of sight burden of $\mathrm{SO}_{2}$. Each spectrum provides a point on the scanned cross section. The cross section area is integrated, multiplied by the wind speed and converted to a mass flux $\left(\mathrm{kg} \mathrm{s}^{-1}, \mathrm{t} \mathrm{d}^{-1}\right)$. 


\subsubsection{ASTER}

The retrieval of $\mathrm{SO}_{2}$ from ASTER data is based on the general at-sensor radiance expression developed by Realmuto et al. (1994):

$$
L_{s}=\left\{\varepsilon_{g} B\left(T_{g}\right)+\left(1-\varepsilon_{g}\right) L_{d}\right\} t_{a}+L_{u}
$$

where $\varepsilon_{g}$ is the ground emissivity, $\mathrm{B}$, the Planck function, $T_{g}$, the temperature of the ground, $t_{a}$, the transmission of the atmosphere, $L_{d}$, the downwelling radiance and $L_{u}$ the upwelling radiance. The radiative transfer code that utilizes the at-sensor radiance expression is called Map_SO2 and is based on MODTRAN (Berk et al. 1989), Map_SO2 contains spectral band models for twelve atmospheric gas molecules, including $\mathrm{SO}_{2}$. The band models are used in conjunction with a user defined atmosphere and the spectral emissivity of the ground to calculate the at-sensor radiance. The upwelling and downwelling radiance and transmissions of the atmosphere are derived from the user defined atmosphere. However, additional information is required, such as plume altitude (top and bottom) and thickness, to help constrain this inverse problem. With the atmospheric radiosonde profile and cloud geometry defined this leaves only the ground temperature, emissivity and $\mathrm{SO}_{2}$ burden unresolved. To calculate the $\mathrm{SO}_{2}$ concentration the 'true' ground temperature and emissivity must be calculated using the TIR ASTER bands that lie out of the $8.6 \mu \mathrm{m} \mathrm{SO}$ absorption feature (bands 13 and 14). An area of interest is selected outside of the plume and the emissivity and true ground temperature are calculated from the spectra of the corresponding pixels in the area of interest. The 'apparent' ground temperature is calculated using the TIR ASTER bands that lie within the $\mathrm{SO}_{2}$ absorption feature (bands 10, 11 and 12). It is the difference between the apparent and true ground temperatures that allows the $\mathrm{SO}_{2}$ load to be determined, as the temperature difference is a linear function of the $\mathrm{SO}_{2}$ burden (Realmuto. 2000; Watson et al. 2004). The result is an $\mathrm{SO}_{2}$ map of the original image with the units of $\mathrm{SO}_{2} \mathrm{~g} \mathrm{~m}^{-2}$. 


\subsection{Results}

Figure 1a illustrates the location of Lascar volcano in relation to the direction of the plume and the UV instrument locations. The raw TIR ASTER image did not indicate a plume being emitted from Lascar volcano (Figure 2.1b, bands 10, 11, and 12). With the use of the IR retrieval technique an $\mathrm{SO}_{2}$ map was produced from the raw ASTER data that showed a weak $\mathrm{SO}_{2}$ plume from Lascar (Figure 2.2).

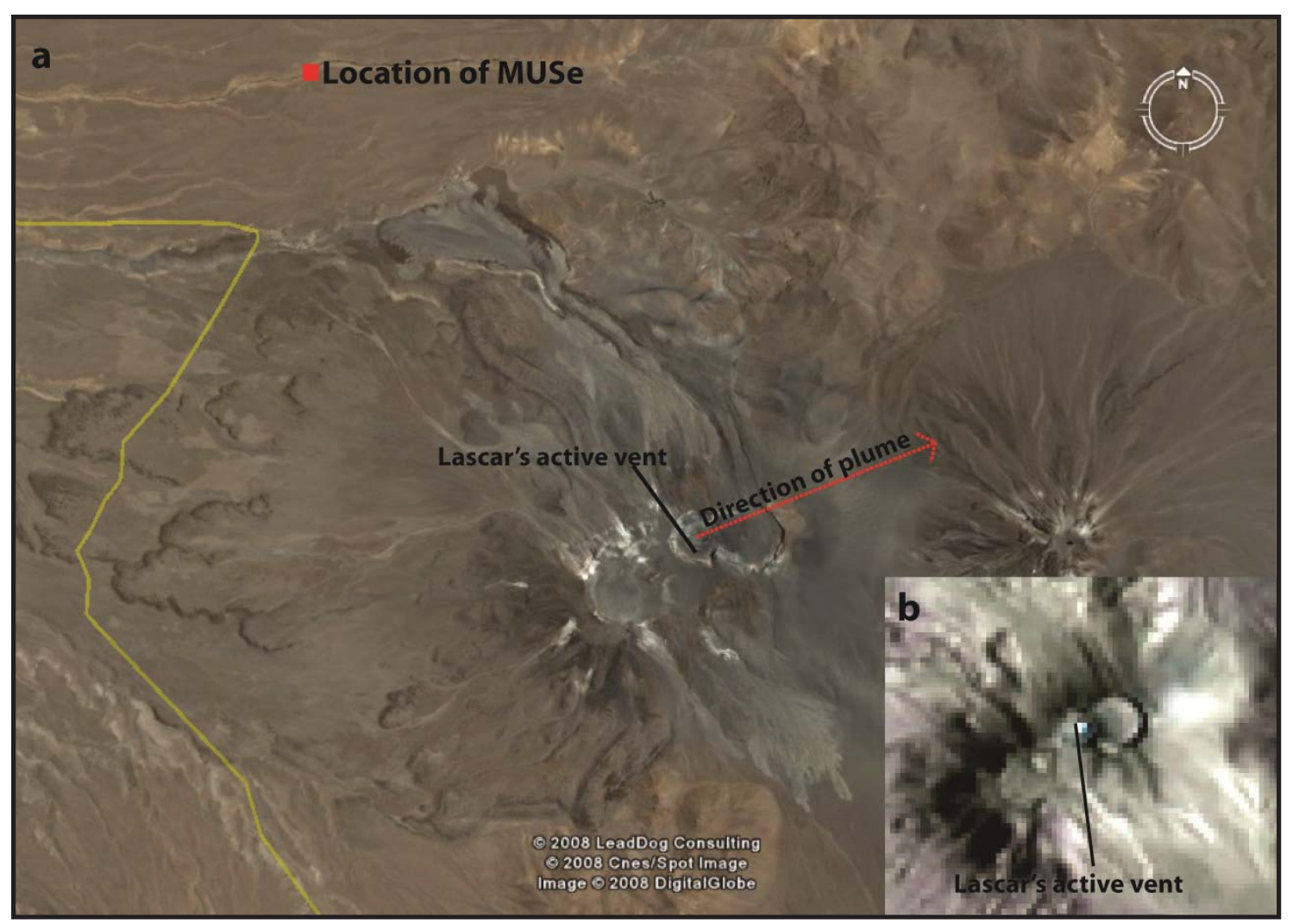

Figure 2.1: 1a illustrates Lascar volcano with the location of where the MUSe measurements were taken, and the direction of the plume, labeled. $1 \mathrm{~b}$ is the TIR ASTER image (bands 10,11 and 12) of Lascar volcano for December $7^{\text {th }}$, 2004. There is no visible plume in the image. 


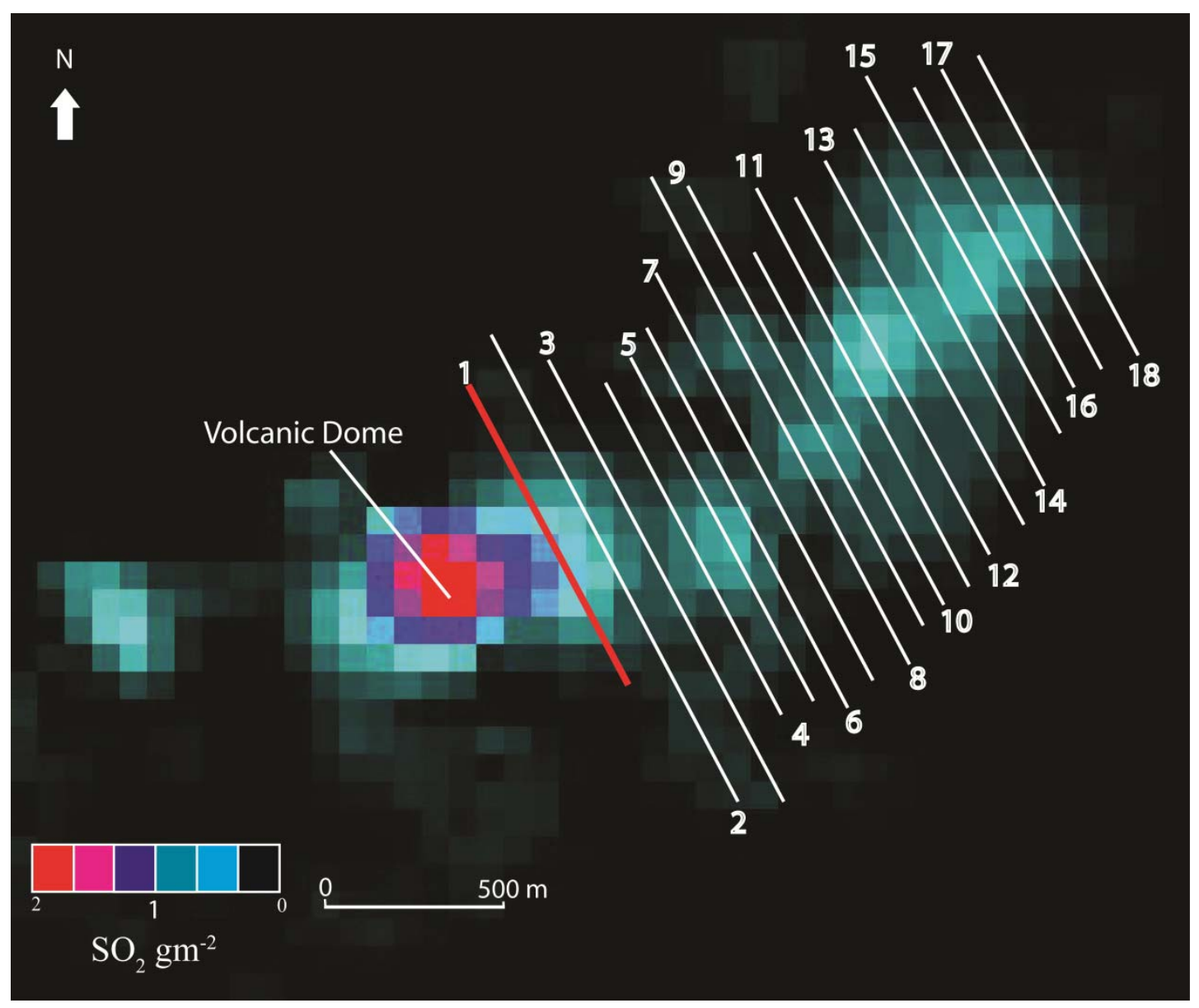

Figure 2.2: The Map_SO2 image produced from the ASTER data. The location of the eighteen transects taken across the plume is illustrated. Transect 1 (in red) also indicates the location of the MUSe transects.

Eighteen parallel transects were taken across the ASTER image, roughly one every pixel (Figure 2.2) and $\mathrm{SO}_{2}$ fluxes were calculated for these transects. Transect 1 was taken at the location where the MUSe observations were made using the azimuth of the observations to direct the transect. This particular transect was acquired at 11:47:18. The subsequent ASTER transects were taken downwind, which represents a negative time step (i.e. earlier in time). Using the wind speed $\left(9 \mathrm{~m} \mathrm{~s}^{-1}\right)$, the pixel size and the time the image was acquired, the time at which the transects would have been obtained by the MUSe (before moving downwind) has been calculated using transect 1 as a starting point. The dashed line indicates when the ASTER image was acquired 
(Figure 2.3). The wind speed was calculated by tracking the plume downwind. Two points were identified from a topographic map that could be easily identified in the field, from the location where the MUSe measurements were conducted. The time it took for a portion of the plume (a puff) to move from point 1 to point 2 was calculated (Williams-Jones et al. 2006). Knowing the distance between the two points and the time it took for the plume to travel between them, the wind speed was calculated. This method helps improve the errors in the flux calculation, making the wind speed more accurate.

Only one measurement from each instrument occurs at the same time (1 second apart) and intersect one another (Figure 2.3b, circled point), ASTER transect 6 and MUSe instrument 1, transect 12. These two transects are presented in Figure 4 and show some similarities in the peaks and troughs of $\mathrm{SO}_{2}$, although due to the different viewing geometries similarity is not necessarily expected. The $\mathrm{SO}_{2}$ fluxes derived from the MUSe and ASTER transects are estimated at $203 \mathrm{t} \mathrm{d}^{-1}$ and $163 \mathrm{t} \mathrm{d}^{-1}$ respectively. 


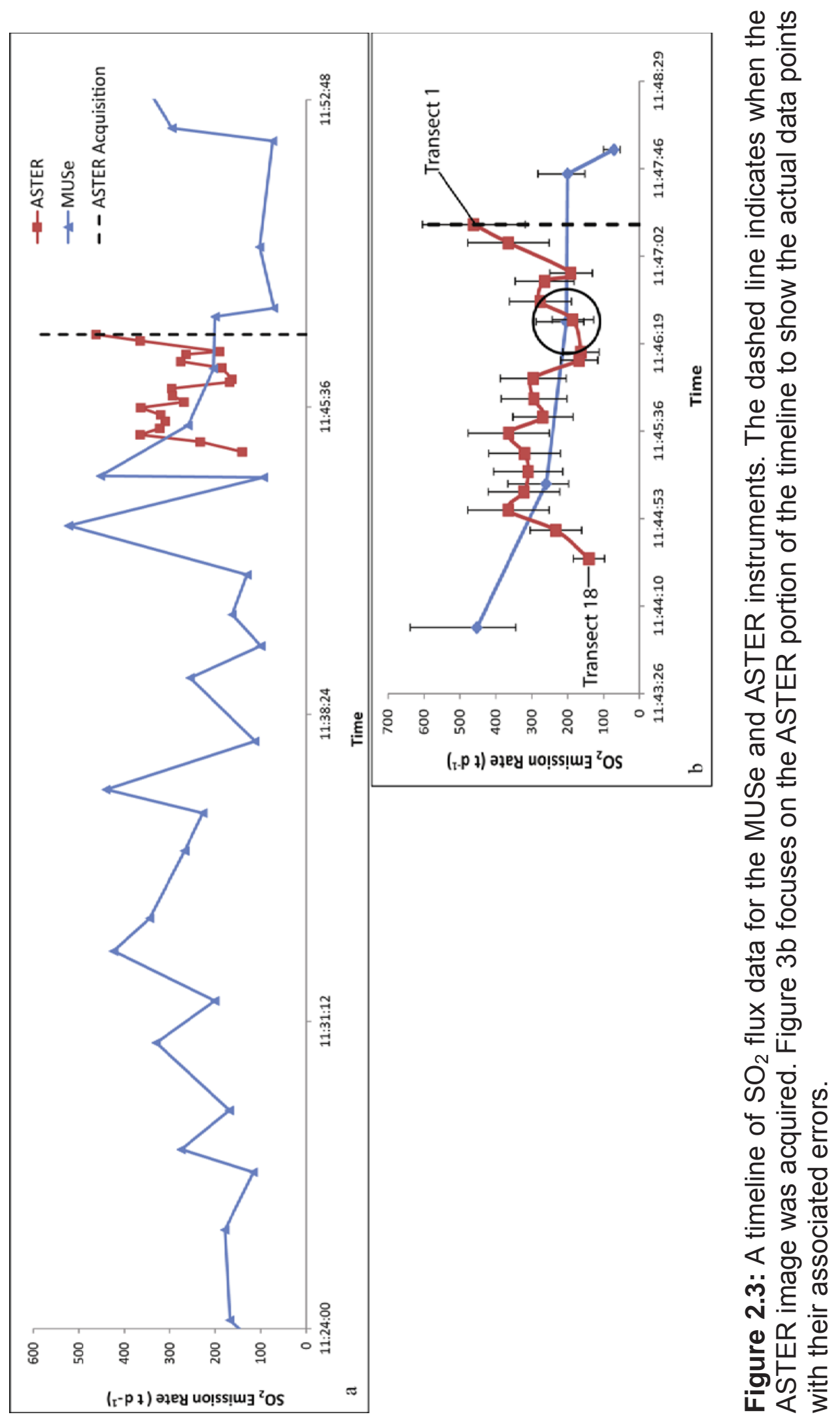




\subsection{Discussion}

The results for the comparison between the MUSe and ASTER instruments are promising, indicating that the two techniques are capable of producing comparable measurements of $\mathrm{SO}_{2}$ fluxes. The ASTER timeline has eighteen data points versus two MUSe data points (Figure 2.3b) acquired during the three minute period of time. Both MUSe data points agree within error with the ASTER flux calculations. They have fluxes of 260 and $203 \mathrm{t} \mathrm{d}^{-1}$ (MUSe) as compared to closest ASTER data point $\left(310\right.$ and $\left.167 \mathrm{t} \mathrm{d}^{-1}\right)$. An error of $+/-31 \%$ was applied to the ASTER data (Henney 2006). An error of $+41 \%$ and $-24 \%$ was applied to the MUSe data (Rodriguez et al. 2007).

The important variables in the ASTER retrieval process are the plume altitude and thickness, and the local atmospheric conditions. Any uncertainties regarding these factors will generate errors that propagate throughout the data produced. The plume altitude is a significant factor in that it defines the temperature contrast between the ground and the plume (Realmuto. 2000). The plume altitude is often assumed to be the altitude of the volcano. However in this case there is visual evidence of the plume altitude and thickness making these parameters more accurately defined than they would be if making a rough estimation. Realmuto et al. (1997) estimated that uncertainties in the plume dimension gave rise to a $+/-10 \%$ error. A radiosonde profile is utilized in the model to provide details of the atmospheric conditions. An error of $\pm 25 \%$ is attached to the use of radiosonde data (Realmuto and Worden. 2000). Such a large error is partly due to the fact that radiosonde data are often collected far from the location of the target volcano where atmospheric conditions may differ. Other errors in the retrieval are associated with instrument calibration and sensitivity, and the accuracy of the Map_SO $\mathrm{SO}_{2}$ procedure. The accuracy of the mapping procedure is dependent on the precision of radiance measurements and any estimation made in describing the transfer of radiation from the ground to the sensor (Realmuto et al. 1997). Studies that have been conducted using 
thermal infrared airborne sensors, such as TIMS (and simulated ASTER and MODIS data), to retrieve the $\mathrm{SO}_{2}$ column abundance have had a minimum error budget that range between $15 \%$ and $25 \%$ (Realmuto et al. 1994; 1997; Realmuto. 2000; Realmuto \& Worden. 2000). An overall root mean square (RMS) error of $31 \%$ was calculated and applied to the results here. The error calculated for the MUSe is based on the calibration errors, processing errors such as smoothing and filtering, error in the wind speed calculation, scattering effects, and the estimation of plume width. The MUSe errors are described in more detail by McGonigle et al. (2003) and Rodriguez et al. (2008).

The MUSe captured data by scanning the plume in a stationary position from the side, top to bottom, whereas ASTER looks at the plume from above. Data obtained from two different slices of the plume may not allow for a good comparison of such techniques through the use of transect comparison. This is evident in Figure 3.4 with the comparison of the ASTER and the MUSe transects. The two transects differ in width with the ASTER transect being 1300 $\mathrm{m}$ and the MUSe $638 \mathrm{~m}$. This reflects the different orientations in which the plumeis viewed and data is collected by each instrument. The plume cannot be viewed from above by the MUSe due to the lack of UV light available from this orientation for the instrument to work efficiently. However, the plume could be traversed from below, and transects could be taken perpendicular to the plume/wind direction (by vehicular and airplane/helicopter techniques, for example). This would allow for a better comparison between the MUSe and ASTER data. It should also be noted that the spatial resolution of the two instruments differs, with ASTER TIR pixels being $90 \mathrm{~m}$ and the MUSe field of view is $176 \mathrm{~m}$. 


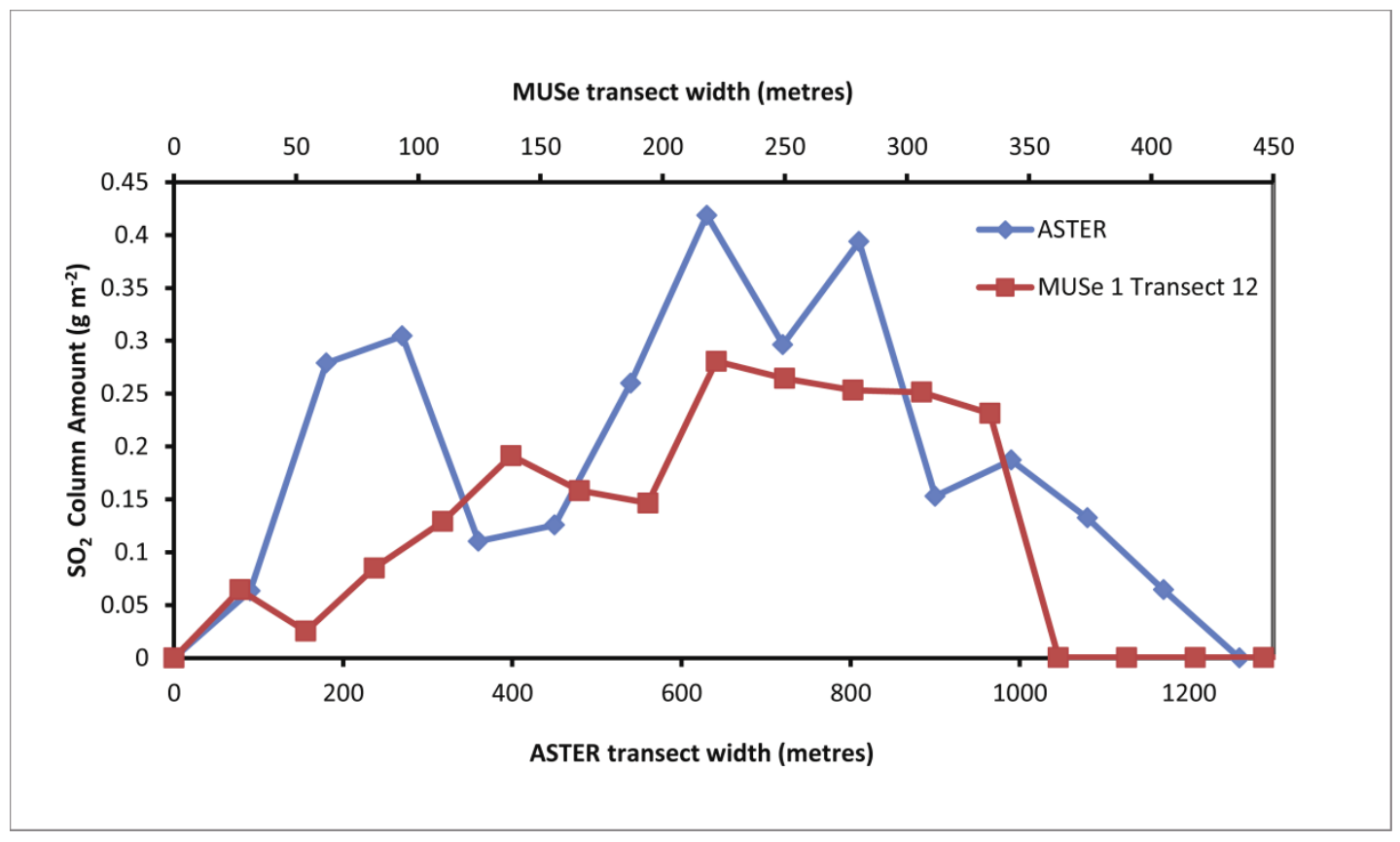

Figure 2.4: $\mathrm{SO}_{2}$ levels measured in ASTER transect 6 , and by MUSe instrument 1 , transect 12 are compared.

Generally, as a plume moves downwind from the vent any magmatic signal (e.g. from convective overturn) is reduced by atmospheric dynamics (Boichu et al. 2010). This is evident from the timeline with ASTER transect 1, which was taken close to the vent, being higher in $\mathrm{SO}_{2}$ concentration than transect 18 , which is taken at the furthest point downwind from the vent visible in the ASTER image. Transect 18 was in the position of transect 1, 4 minutes and 15 seconds before it reached its location in the ASTER image. The daily emission rate of the volcano is taken from the transect that is taken closest to the vent. In this case it is transect 1 , which is also the location of where the MUSe intersects the plume. The ASTER $\mathrm{SO}_{2}$ fluxes in Figure $2.3(\mathrm{a}$ and $\mathrm{b}$ ) will therefore be underestimated the further downwind you go from transect 1. Other sources of loss in the downwind plume observations, relative to the at-vent measurements include dispersion (and subsequent loss of detectability), dry deposition and chemical loss through conversion to sulphate aerosol. However due to Lascar's high altitude and dry atmosphere loss is minimal, making it ideal for this study (Mather et al. 2004; Rodriguez. 2007). 
Comparing the averages of the two instruments provides another approach at looking at the data. Figure 2.5 illustrates the average MUSe flux $\left(229 \mathrm{t} \mathrm{d}^{-1}\right)$, ASTER flux $\left(277 \mathrm{t} \mathrm{d}^{-1}\right)$ and the flux from ASTER's transect $1\left(462 \mathrm{t} \mathrm{d}^{-1}\right)$. The MUSe and ASTER average fluxes are in close agreement. Comparing the average ASTER flux to the average MUSe flux is problematic because the average ASTER flux is calculated from the entire plume, including the transects where $\mathrm{SO}_{2}$ is underestimated and the magmatic signal is lost. The MUSe average, however, is calculated from constant measurements at one particular transect of the plume close to the vent. Instead of the average, ASTER's transect 1 can be compared to the MUSe average because it represents where the MUSe transects were taken. The flux at ASTER's transect 1 is much higher than the MUSe average but they lie within each other's error margins.

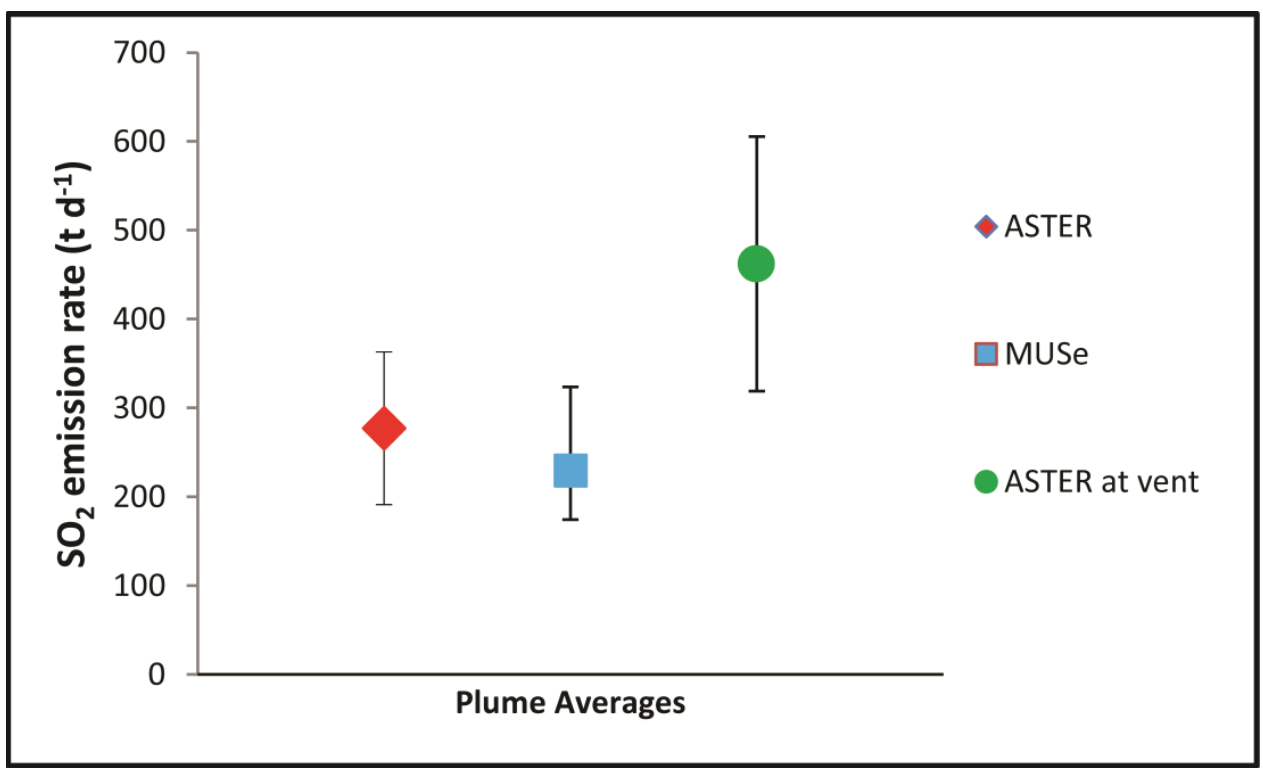

Figure 2.5: Average $\mathrm{SO}_{2}$ fluxes determined using the MUSe and ASTER instruments. The $\mathrm{SO}_{2}$ flux for ASTER transect 1 is also displayed. 


\subsection{Conclusions}

When compared to the MUSe instrument, ASTER has proved to be a reliable detector of $\mathrm{SO}_{2}$ at passively degassing volcanoes (Urai. 2004 and this study). Under the ideal circumstances presented at Lascar, mini-UV DOAS instruments acquire measurements that agree within error with ASTER observations. The advantage of acquiring imagery (as opposed to slicing the plume) is shown by the better temporal resolution of the ASTER timeline, although high temporal resolution ground based data has been obtained without satellite imagery (Boichu et al. 2010; McGonigle et al. 2009). Further attempts at validation, for both ASTER and OMI are required to fully assess the reliability of spaceborne observations of passive degassing from volcanoes. 


\section{Chapter 3}

\section{Visual observations of volcanic plumes using Principal Components Analysis (PCA) and Decorrelation Stretch (DCS) Enhancements on ASTER Thermal Infrared Data}




\subsection{Introduction}

Remote sensing is an important tool for detecting and monitoring volcanic plumes for hazard mitigation. Volcanoes emit a variety of products such as sulfur dioxide, silicate ash and water vapor, all of which can be detected by remote sensing techniques. However, these plumes are not always visually discernible with raw thermal infrared (TIR) data, which is problematic when attempting to select Advanced Spacebourne Thermal and Reflection Radiometer (ASTER) images for $\mathrm{SO}_{2}$ plume mapping (Chapters 4 and 5 ). Hence, other image processing techniques must be investigated to determine if the plume truly exists. Not only do image enhancements locate the cloud and its boundaries, but they also determine the plume's composition. Knowing the composition is also important for the MAP_SO2 analysis. If the plume is ash rich it will mask $\mathrm{SO}_{2}$, making it difficult for the algorithm to quantify the $\mathrm{SO}_{2}$. The end result is then less accurate as ash is mapped as an excess of $\mathrm{SO}_{2}$. Typically images with ash rich plumes are not good candidates to be processed with the MAP_SO2 algorithm (McCarthy et al. 2008).

The primary result of an image enhancement technique is to increase the visual interpretability of the data. Two enhancements that have successfully been used in volcanic remote sensing studies are the decorrelation stretch (DCS) and principal components analysis (PCA). These techniques have previously been used for identifying the composition of a plume or distinguishing ash from meteorological clouds.

Rose and Ramsey (2010) used a decorrelation stretch analysis of ASTER bands 14,13 and 11 to view a plume from Kliuchevskoi volcano, Russia. When viewing the bands in $R(14,) \mathrm{G}(13)$, and $\mathrm{B}(11)$ it was noted that $\mathrm{SO}_{2}$ appears yellow, silicate ash appears red to orange and $\mathrm{H}_{2} \mathrm{O}$ appears blue to cyan in color. Dean et al. (1994) used principal component analysis techniques with data from the Advanced Very High Resolution Radiometer (AVHRR) to look at a 
plume emitted from Redoubt volcano, Alaska, and distinguished it from the background and meteorological clouds. Using a PCA and a composite of 3 of the AVHRR bands, the maximum extent of the plume was visible along with its shadow and meteorological clouds. They also determined which portions of the plume were opaque or transparent.

Several studies have been carried out to assess ash clouds using the Geostationary Operational Environmental Satellite (GOES) and the Moderate Resolution Imaging Spectrometer (MODIS) (Hillger et al. 2002; Ellrod et al. 2003; Ellrod 2004). Ellrod (2004) used a PCA to analyze GOES imagery for 6 weak eruptions using images with and without the $12 \mu \mathrm{m}$ band. This comparison was carried out due to the replacement of the $12 \mu \mathrm{m}$ band with a band sensing the $13 \mu \mathrm{m}$ region of the electromagnetic spectrum on GOES satellites launched after GOES 11. This replacement sensor was not used to detect ash. Ellrod (2004) found that the PCA transformation results were excellent when using the $12 \mu \mathrm{m}$ band, but they were not always capable of distinguishing volcanic plumes from meteorological clouds without it. MODIS has proved beneficial using the PCA to look at ash clouds from volcanoes especially since more bands that detect ash are available when compared to GOES. However, the PCA has not specifically been used to identify $\mathrm{SO}_{2}$ plumes from passively degassing volcanoes.

The purpose of this portion of the study is to carry out PCA transformations and decorrelation stretch enhancements on a variety of images that contain volcanic plumes. Results will be compared to determine which method is more applicable for identifying volcanic $\mathrm{SO}_{2}$ plumes in ASTER imagery. The images selected for this analysis will contain plumes ranging in size, composition, $\mathrm{SO}_{2}$ concentration and plume heights, from a variety of volcanoes. 


\subsection{Methodology}

\subsubsection{Principal Component Analysis}

PCA is a technique that transforms a dataset into fewer dimensions (components replace the bands) which are uncorrelated yet maintain most of the spectral information contained in the original dataset (Jensen 2005). The first principal component (PC1) contains the greatest amount of information from the original dataset. Successive components contain decreasing amounts of information. The number of output components equals the number of input bands.

PCs are calculated for the 5 ASTER TIR bands to produce an uncorrelated image composed of 5 PCs. To rotate the original axes to the PCA axes a linear transformation using calculated coefficients must occur. The linear transformation is derived from the covariance matrix of the original ASTER TIR dataset. Eigenvalues and eigenvectors are calculated from the covariance matrix. The eigenvalues contain important information and can be used to compute the total variance within each PC. It is important to understand how the information from the 5 TIR bands contributes to the makeup of each new component. This information is calculated using the eigenvalues, eigenvectors and variance to produce loading factors. Table 3.1 illustrates a selection of the loading factors from some of the images in this study. Appendix 1 contains loading factors for all of the images utilized in this study. 
Table 3.1

Examples of loading factors from the key images discussed in the results section.

\begin{tabular}{|c|c|c|c|c|c|c|}
\hline \multirow{2}{*}{$\begin{array}{l}\text { Hawaii } \\
\text { 03-28-2008 }\end{array}$} & \multicolumn{6}{|c|}{ Component } \\
\hline \multirow{4}{*}{ Band } & & 1 & 2 & 3 & 4 & 5 \\
\cline { 2 - 7 } & 1 & 0.447252 & 0.447153 & 0.447233 & 0.447369 & 0.44706 \\
\cline { 2 - 7 } & 2 & -0.09948 & 0.572515 & 0.42691 & -0.25627 & -0.64374 \\
\cline { 2 - 7 } & 3 & -0.82617 & 0.039856 & 0.377458 & -0.00725 & 0.416319 \\
\cline { 2 - 7 } & 4 & 0.131301 & -0.68268 & 0.650093 & 0.16168 & -0.26067 \\
\cline { 2 - 7 } & 5 & -0.30044 & 0.068148 & -0.22942 & 0.841423 & -0.38008 \\
\hline
\end{tabular}

\begin{tabular}{|c|c|c|c|c|c|c|}
\hline $\begin{array}{l}\text { Lascar } \\
12-07-2004\end{array}$ & \multicolumn{6}{|c|}{ Component } \\
\hline \multirow{4}{*}{ Band } & & 1 & 2 & 3 & 4 & 5 \\
\cline { 2 - 7 } & 1 & 0.44702 & 0.447398 & 0.447198 & 0.447365 & 0.447086 \\
\cline { 2 - 7 } & 2 & 0.695028 & 0.205808 & -0.03205 & -0.21497 & -0.65371 \\
\cline { 2 - 7 } & 3 & 0.404855 & -0.21723 & -0.78222 & 0.289687 & 0.30514 \\
\cline { 2 - 7 } & 4 & -0.3151 & 0.207657 & -0.13961 & 0.758719 & -0.5123 \\
\cline { 2 - 7 } & 5 & -0.2322 & 0.816802 & -0.40942 & -0.30672 & 0.131221 \\
\hline
\end{tabular}

\begin{tabular}{|c|c|c|c|c|c|c|}
\hline $\begin{array}{l}\text { Masaya } \\
\text { 02-09-2005 }\end{array}$ & \multicolumn{6}{|c|}{ Component } \\
\hline \multirow{4}{*}{ Band } & & 1 & 2 & 3 & 4 & 5 \\
\cline { 2 - 7 } & 1 & 0.447151 & 0.447283 & 0.447209 & 0.447388 & 0.447037 \\
\cline { 2 - 7 } & 2 & 0.045338 & 0.48563 & 0.424947 & -0.2287 & -0.72748 \\
\cline { 2 - 7 } & 3 & -0.79578 & 0.085448 & 0.49034 & -0.10769 & 0.327727 \\
\cline { 2 - 7 } & 4 & 0.405726 & -0.39698 & 0.433393 & -0.6638 & 0.222126 \\
\cline { 2 - 7 } & 5 & -0.01114 & -0.63183 & 0.43722 & 0.54343 & -0.33792 \\
\hline
\end{tabular}


Table 3.1 Continued

\begin{tabular}{|c|c|c|c|c|c|c|}
\hline $\begin{array}{l}\text { Popocatepetl } \\
12-15-2007\end{array}$ & \multicolumn{7}{|c|}{ Component } \\
\hline \multirow{5}{*}{ Band } & & 1 & 2 & 3 & 4 & 5 \\
\cline { 2 - 7 } & 1 & 0.447211 & 0.447208 & 0.447215 & 0.447292 & 0.447142 \\
\cline { 2 - 7 } & 2 & -0.02674 & 0.519992 & 0.481362 & -0.38191 & -0.59273 \\
\cline { 2 - 7 } & 3 & -0.86041 & 0.139147 & 0.302479 & 0.034584 & 0.384249 \\
\cline { 2 - 7 } & 4 & -0.00928 & -0.64906 & 0.609444 & 0.345341 & -0.29657 \\
\cline { 2 - 7 } & 5 & -0.24269 & 0.298279 & -0.32461 & 0.730494 & -0.46167 \\
\hline
\end{tabular}

\begin{tabular}{|c|c|c|c|c|c|c|}
\hline $\begin{array}{l}\text { Ubinas } \\
\text { 05-08-2006 }\end{array}$ & \multicolumn{7}{|c|}{ Component } \\
\hline \multirow{4}{*}{ Band } & & 1 & 2 & 3 & 4 & 5 \\
\cline { 2 - 7 } & 1 & 0.446831 & 0.447414 & 0.447379 & 0.447374 & 0.447069 \\
\cline { 2 - 7 } & 2 & 0.752846 & 0.133225 & -0.03248 & -0.267 & -0.585513 \\
\cline { 2 - 7 } & 3 & 0.450807 & -0.47484 & -0.59951 & 0.220174 & 0.221878 \\
\cline { 2 - 7 } & 4 & -0.15475 & 0.183048 & -0.30997 & 0.775761 & -0.494633 \\
\cline { 2 - 7 } & 5 & -0.07996 & 0.723249 & -0.58592 & -0.279250 & 0.221878 \\
\hline
\end{tabular}




\subsubsection{Decorrelation Stretch}

The decorrelation stretch (DCS) is similar to a principal component analysis (Gillespie 1992; Alley 1996) and is used to highlight as much discrimination of scene variation as possible. The DCS enhancement is expected to keep the colors and brightness consistent between images that are acquired at different locations and/or times. A DCS image is calculated using 3 of the 5 TIR bands. Bands 14, 13 and 11 are chosen for this study. These bands are chosen due to the volcanic species they detect: band 11 covers the strongest portion of the $\mathrm{SO}_{2}$ absorption feature located at $8.6 \mu \mathrm{m}$ and bands 13 and 14 are used to detect ash and water. The first two steps convert the 3 TIR bands to 3 PCs. The third step normalizes the variances of the eigenvectors, which is key to keeping the color and brightness consistent between images. The final step rotates the data back to the original display axes. The last three steps are described by using the following equation:

$$
T=R^{t} s R
$$

Where $\mathrm{R}$ is the matrix of eigenvectors, $\mathrm{t}$ represents the transpose of the matrix of eigenvectors, $s$ is the normalization vector and $T$ is the final transformation matrix (the final DCS enhanced image). The output is an 8 bit image. 


\subsection{Results}

Images were selected from 13 volcanoes and transformed using PCA and DCS. The twenty five images chosen represent a wide variety of plume sizes, compositions and altitudes. The images also vary in terms of percentage meteorological cloud cover and the ease with which the plume can be identified by the naked eye in the visible or TIR. Table 3.2 lists the volcanoes and the acquisition date of the images. It also provides a description of the plume and information on the resulting PCA and DCS enhanced scenes. The results vary depending on the image. For the PCA this is expected as the transformation is scene dependent. However, for the DCS all the images should be consistent with the plume being easily identified along with the composition (ash, $\mathrm{H}_{2} \mathrm{O}$ vapor or $\mathrm{SO}_{2}$ ). 


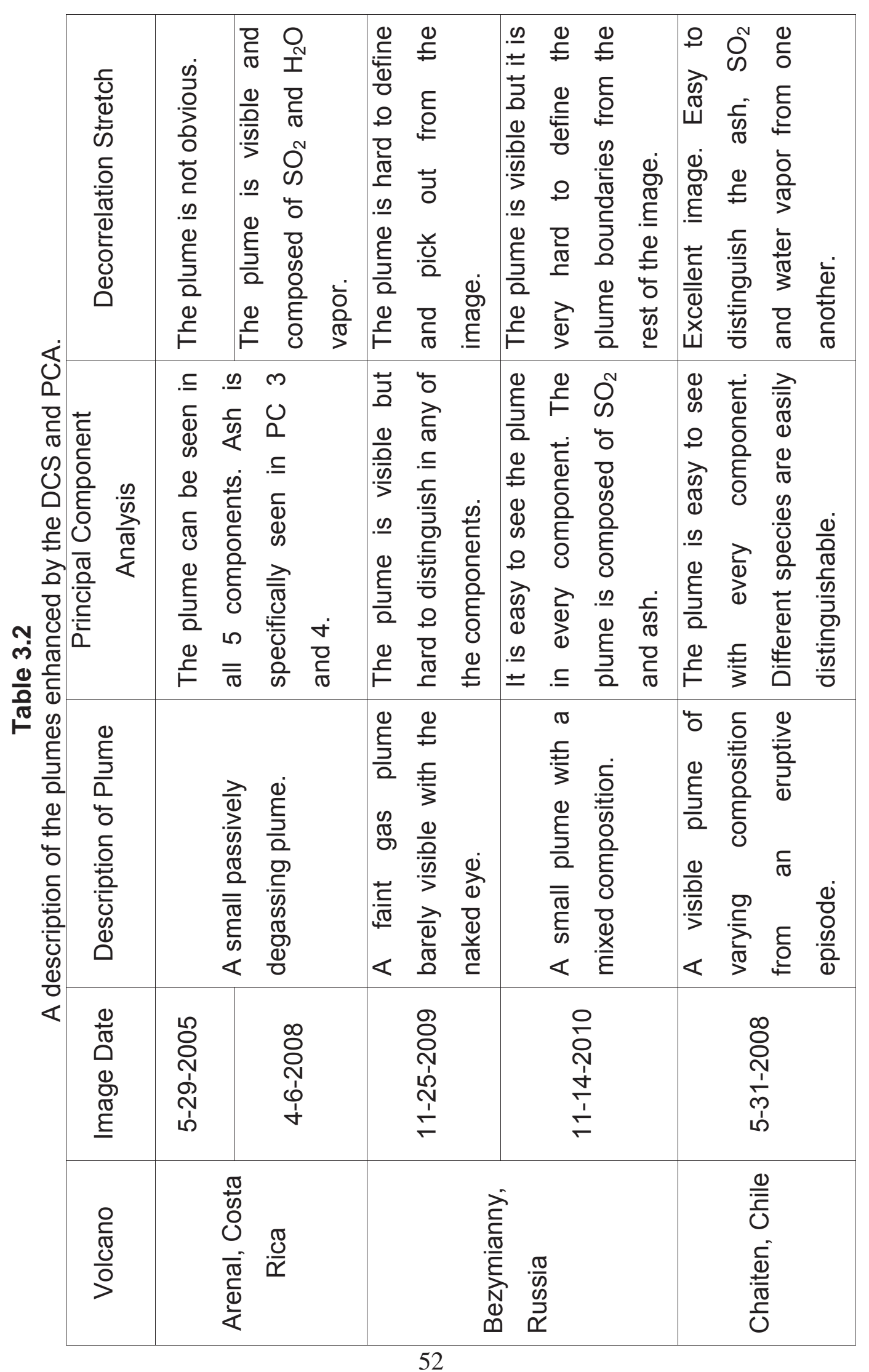




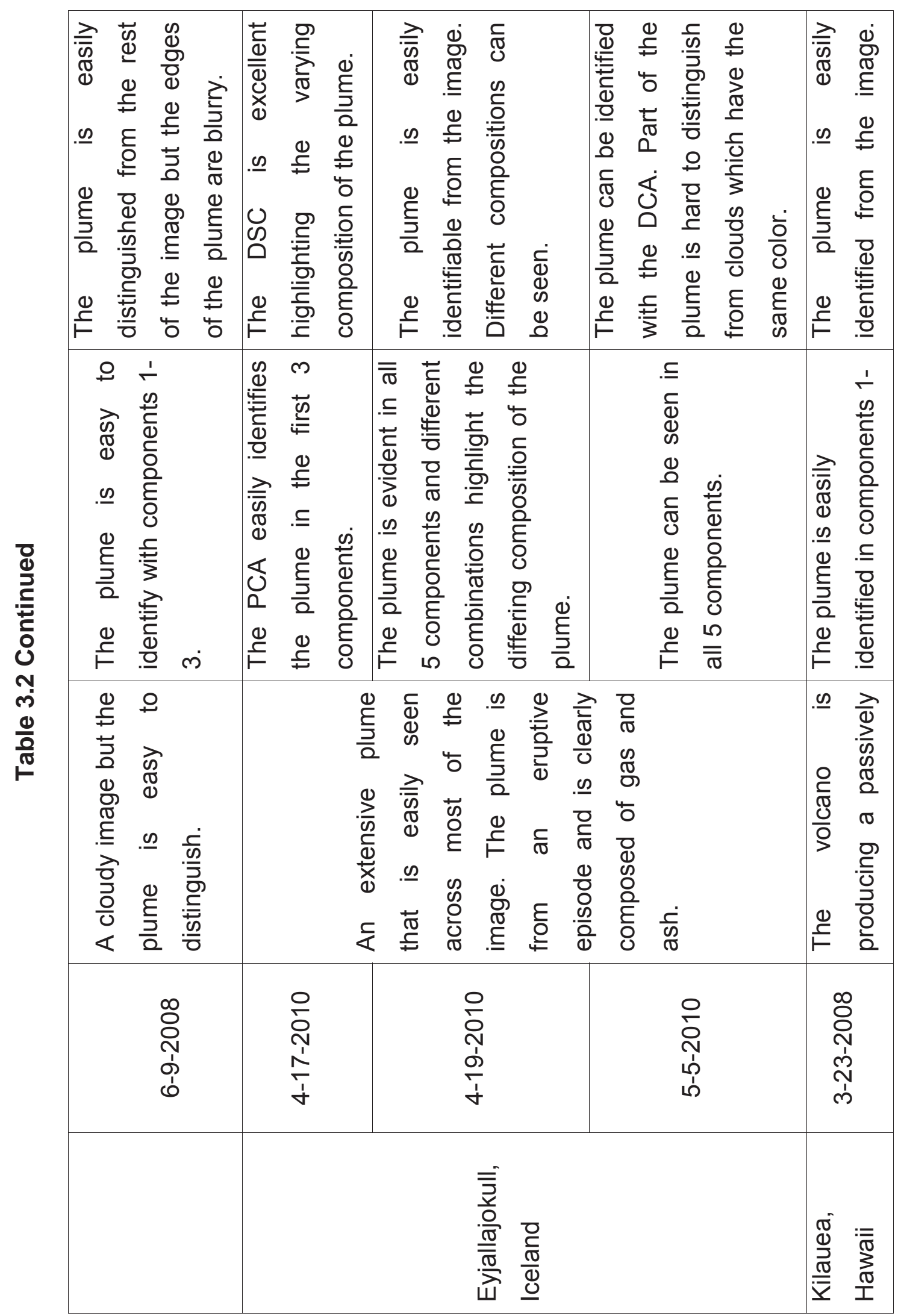




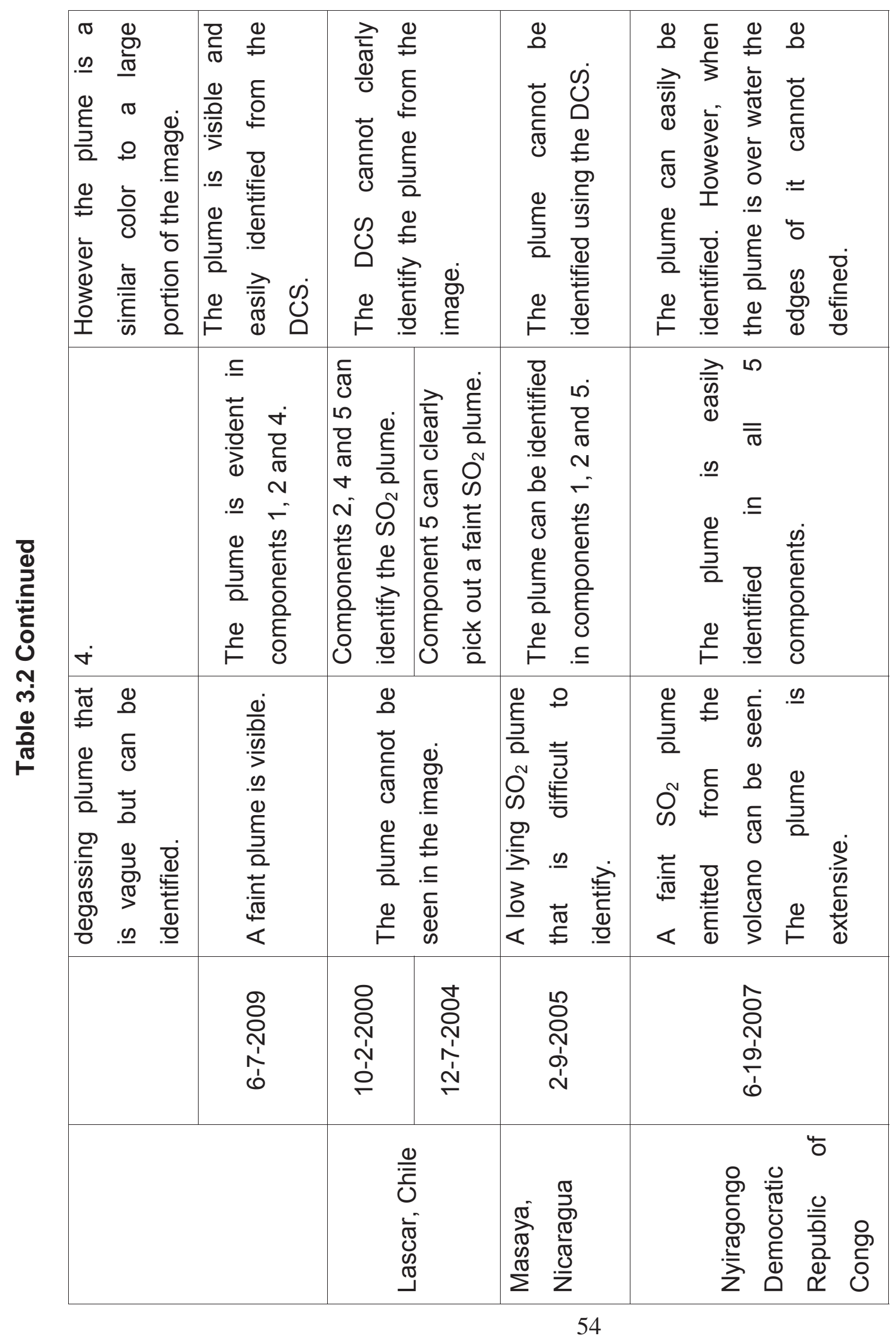




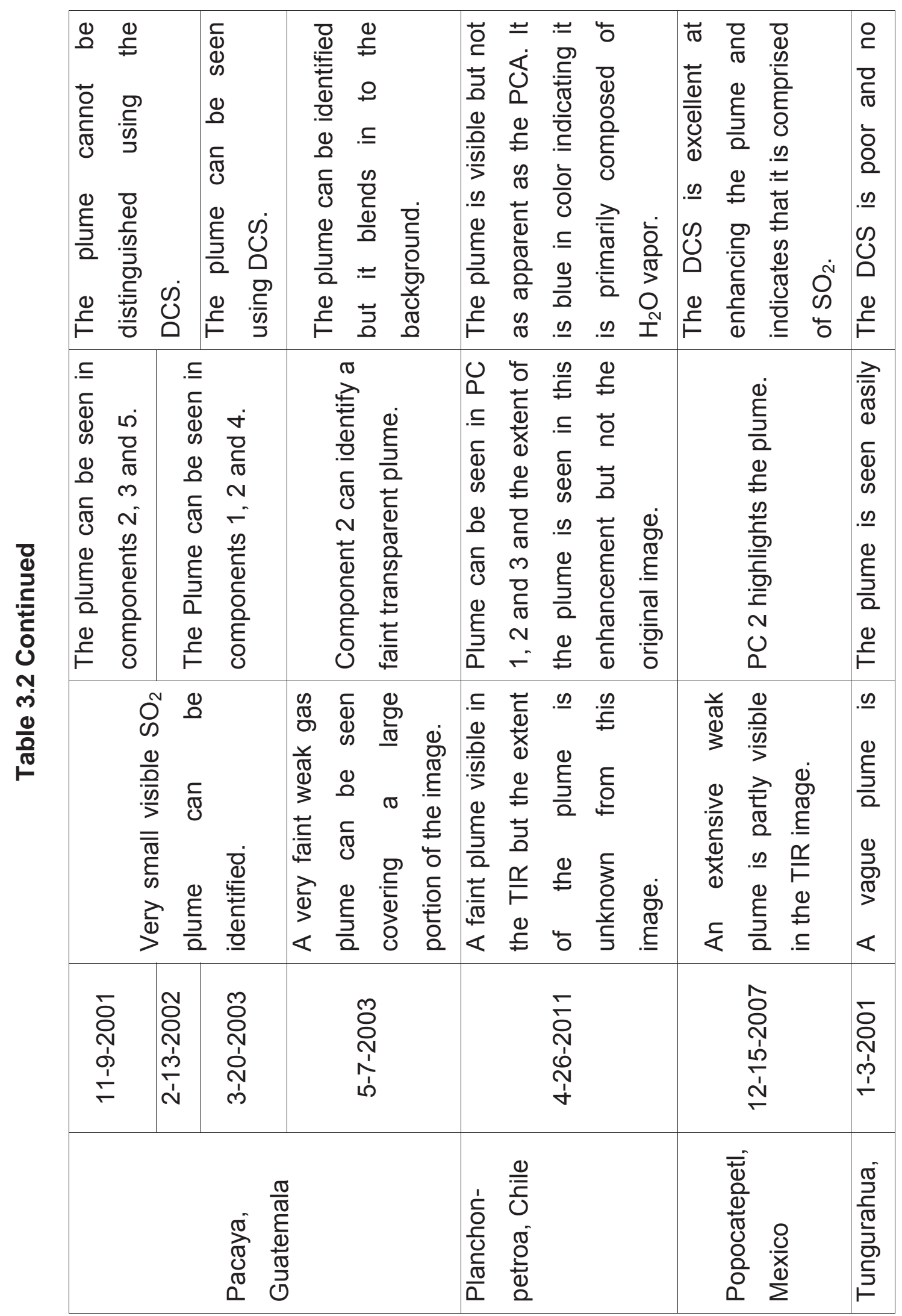




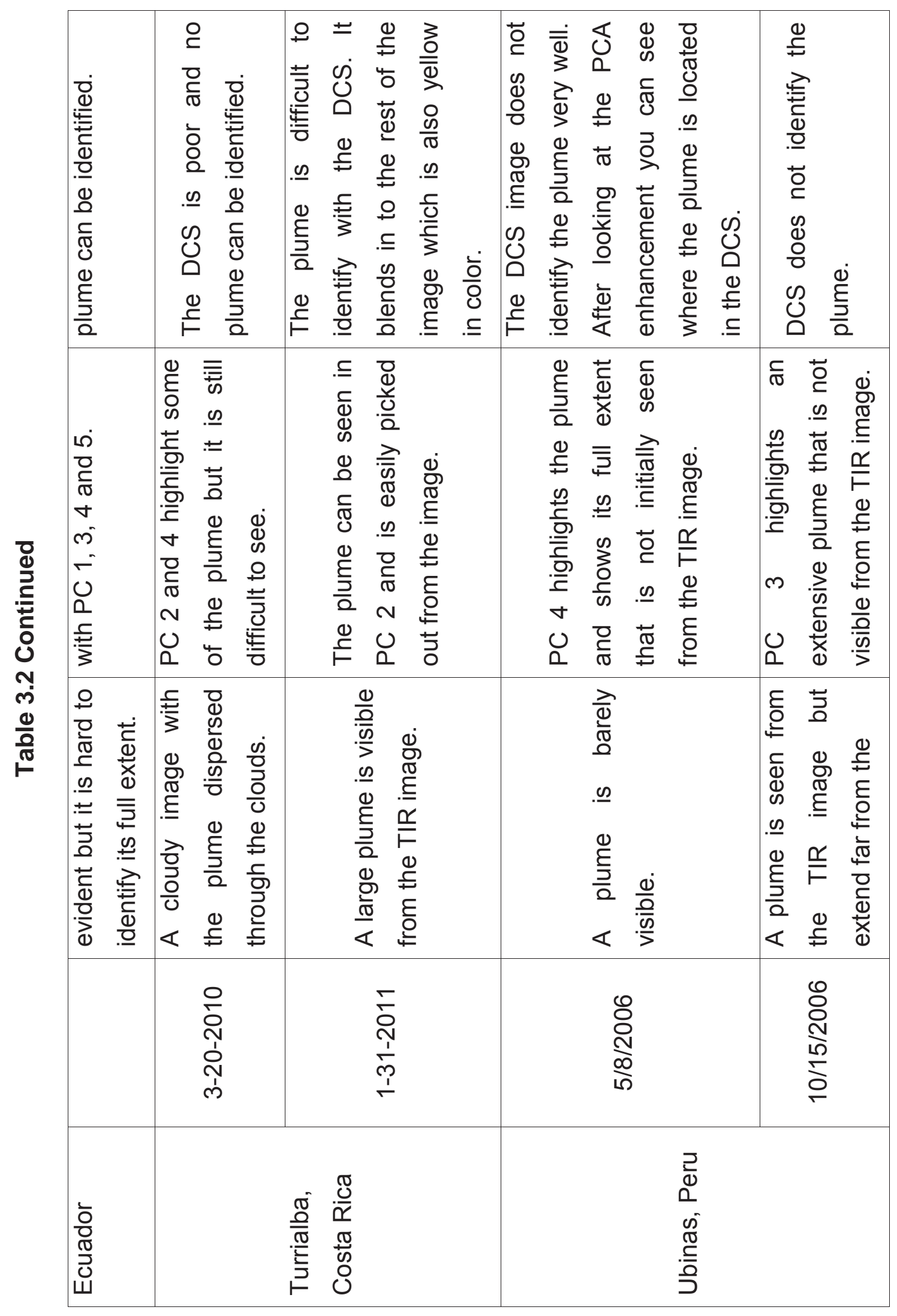


The DSC and PCA transformations produced a wide range of results. When looking at explosive eruptions with large plumes the results for both transformations were excellent and the plumes from these volcanoes (Chaiten, Chile and Eyjafjallokull, Iceland) are easily identified and the composition of the plumes is distinguishable. Figures 3.1 and 3.2 illustrate examples of the plumes from each of these volcanoes viewed in the TIR and both transformations. The plumes are easily seen in the original image and so the enhancements are not necessary. In the Chaiten image, part of the plume is not easily seen in the TIR image at first glance. The TIR image shows a plume dispersing to the east, but in both enhancements it is evident that the plume is larger and flows not only to the east but extends to the south east (Figure 3.2). Both enhancements clearly show that the plume is made up of gas and ash. In the DCS (Figure 3.2c) the $\mathrm{SO}_{2}$ is yellow in color while the ash is pink in color. The PCA (Figure 3.2b) illustrates the differing components of the plume but due to the enhancement being scene dependent no set color represents $\mathrm{SO}_{2}$ or ash. Using the DCS stretch it is known that in the PCA ash is red in color and $\mathrm{SO}_{2}$ is blue for this particular image. 


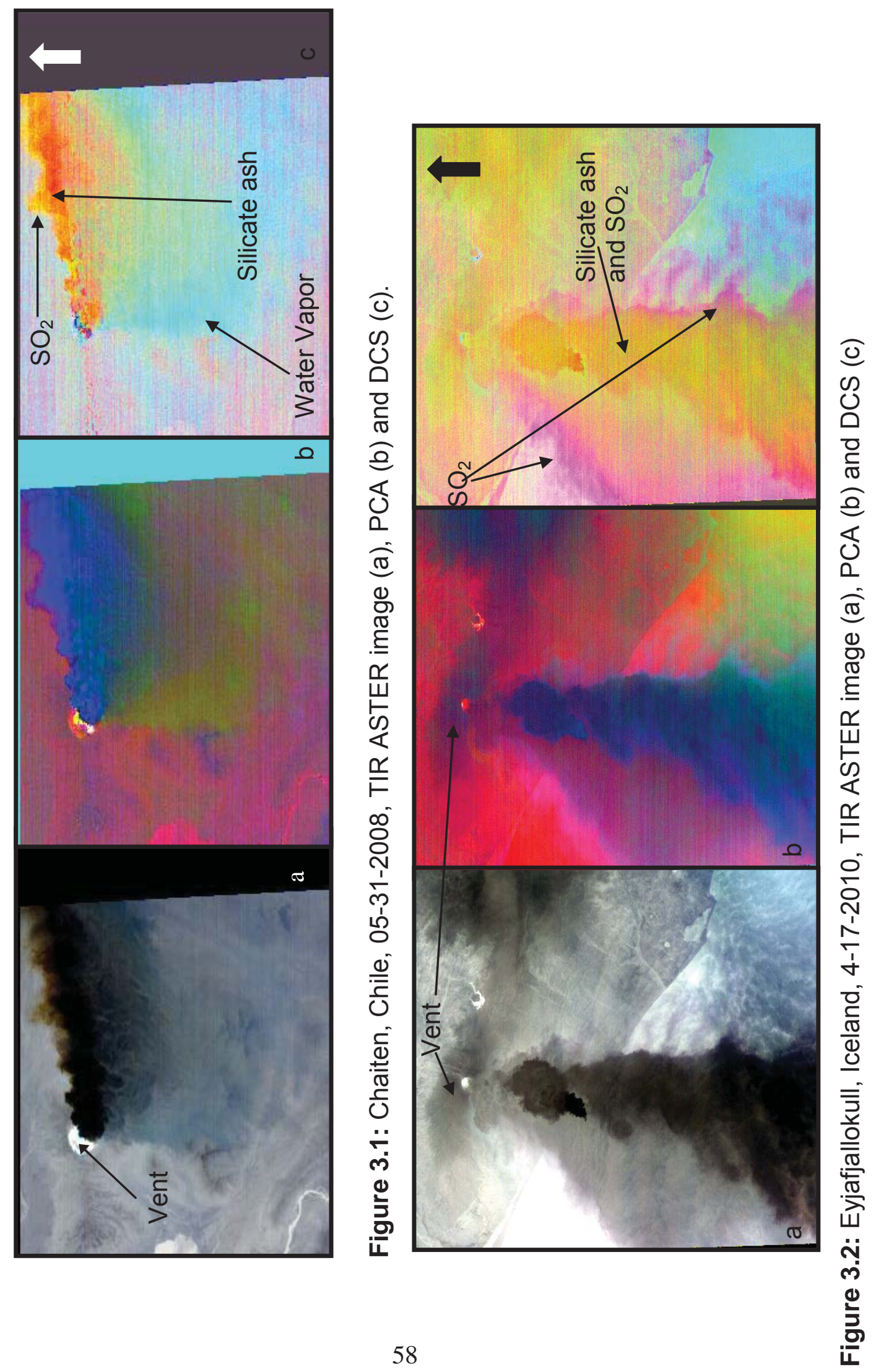


For plumes from volcanoes that are passively degassing and composed primarily of $\mathrm{SO}_{2}$, the PCA and DCS give mixed results, with the PCA generally producing a more interpretable image than the DCS. Figures 3.3 and 3.4 illustrate large passively degassing plumes at a high altitude from Ubinas, Peru and Popocatepetl, Mexico respectively. At Ubinas, the plume is not identified from the TIR image. After a DCS enhancement, the plume is still not evident. Principal Component (PC 3) easily identifies the plume and is well defined. The loading factors indicate that PC 3 is primarily composed of information from band 10 which lies in the $\mathrm{SO}_{2}$ absorption feature (Table 3.1). The PCA and DCS images of Popocatepetl are both excellent in highlighting the plume. The DCS highlights the plume in yellow indicating that it is comprised of $\mathrm{SO}_{2}$. The plume is evident in components 2 and 4 of the PCA enhancement with the factor loadings indicating that the first 3 bands make up PC 2 and bands 10 and 12 make up PC 4 (Table 3.1). All these bands lie across the $\mathrm{SO}_{2}$ absorption feature. Turrialba is interesting (Figure 3.5) in that its plume is barely visible in the PCA and DCS enhancements. The PCA provides a better enhancement because the plume does not blend with the rest of the ASTER image as it does in the DCS output. The plume in the PCA is dark red in color. The background of the image is also red, but a lighter shade of red making it possible to distinguish the plume from the background (Figure 3.5b). With the DCS the plume boundaries are uncertain as the $\mathrm{SO}_{2}$ plume is yellow in color as is the bulk of the image (Figure3.5c). 

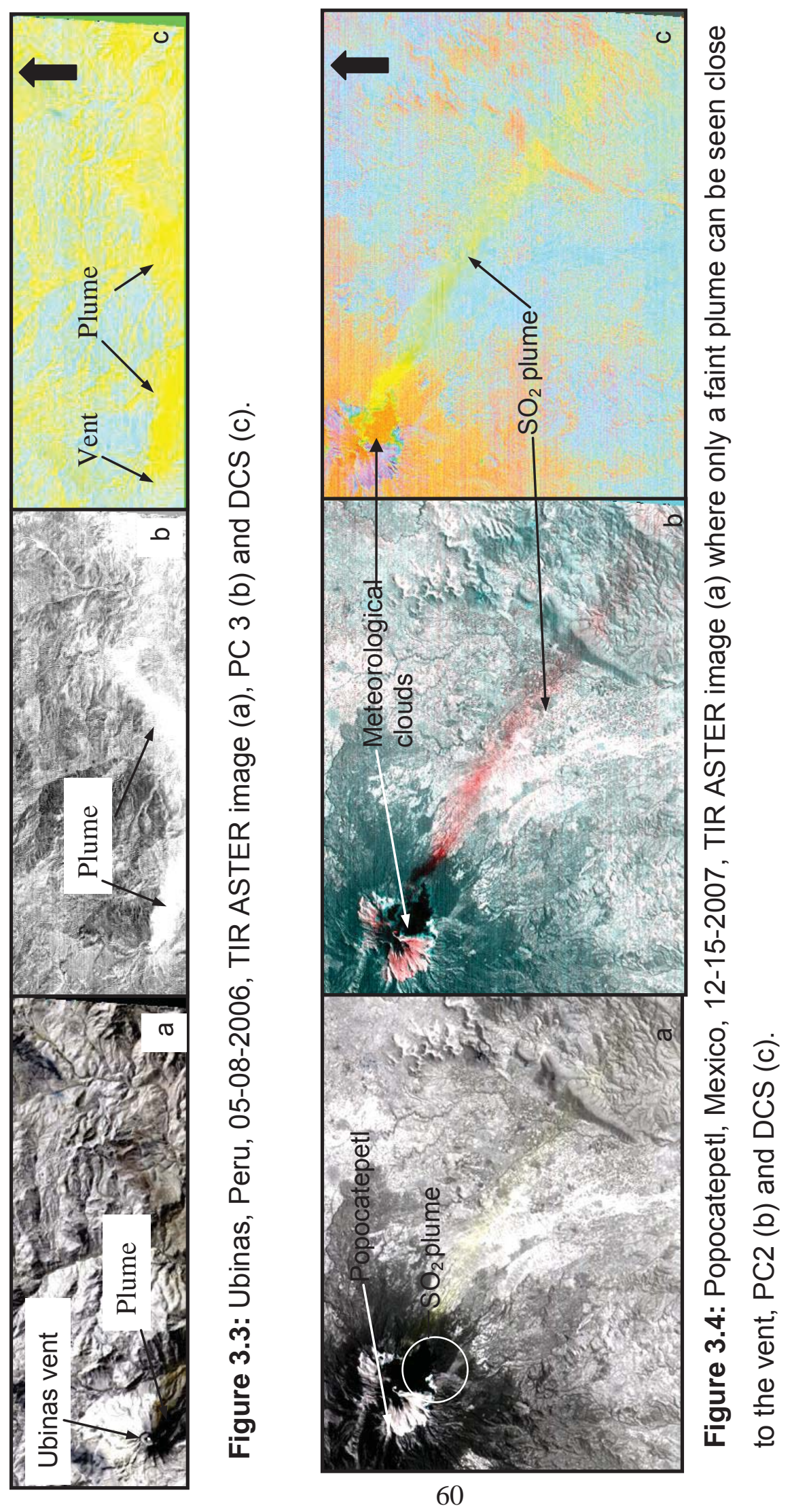


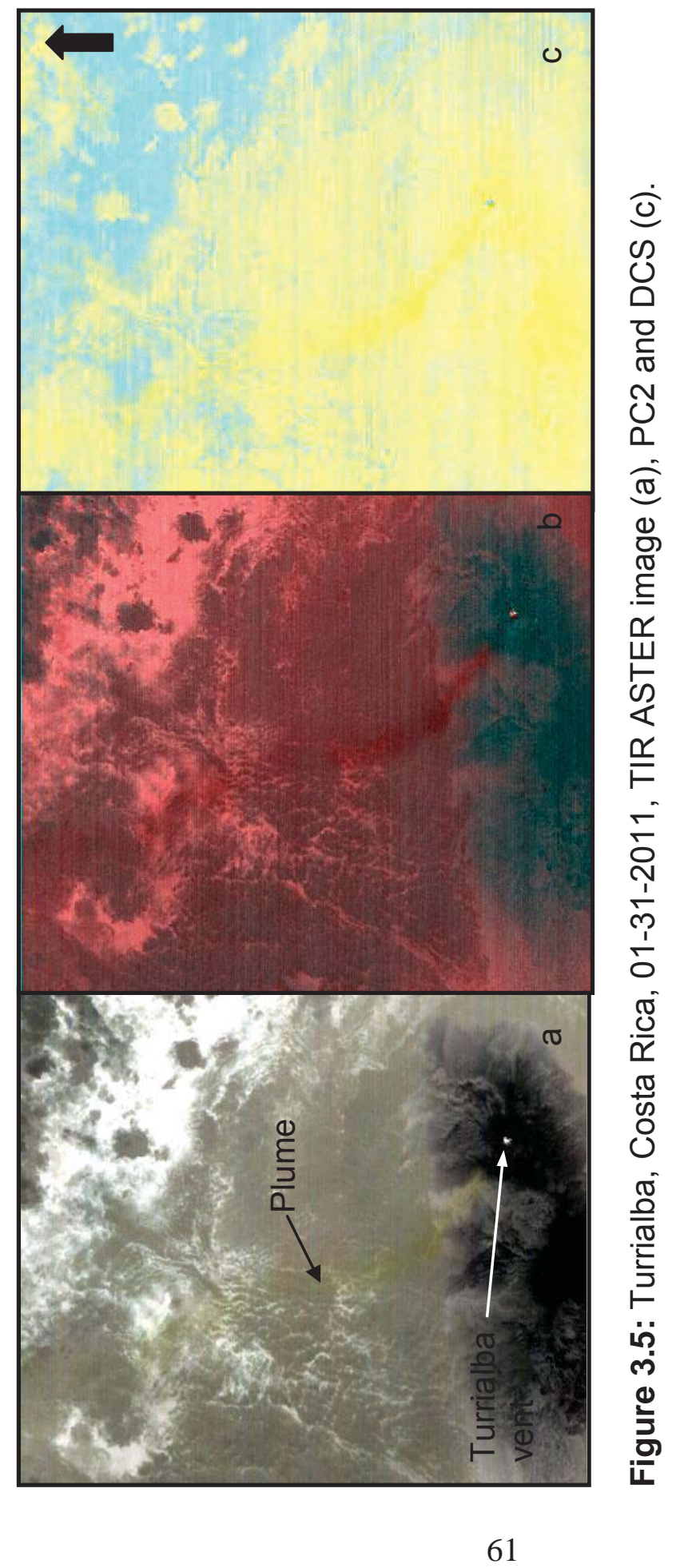


Weak plumes and low lying plumes from volcanoes such as Lascar, Chile (Figure 3.6) and Masaya, Nicaragua (Figure 3.7) did not show visible plumes using the DCS, but a faint plume from both these volcanoes was highlighted in the PCA. At Lascar, no plume can be seen in the TIR image acquired on 12 December 2004, and the DCS does not show a plume is present. The majority of the DCS image is yellow in color and does not show any variation between features in the image such as rock types or vegetation. The PCA enhancement highlights a small plume in PC 5. The factor loadings show that this component is made up of bands 10 and 12 (Table 3.1). The plume appears thin and fairly transparent with the ground being viewed through it. PC 5 typically contains noise and so it might be that the plume is classified as noise. The plume at Masaya is characteristically low lying and often difficult to view (Delmelle et al. 2002). Using both the enhancement techniques does not assist in plume identification. As with the Lascar image, described above, the DCS is mostly a yellow hue with no distinction between different features. The PCA image does distinguish the plume better than the DCS with PCs 2 and 5 mapping the plume. Again, the plume is thin and the ground can be seen through it. The loading factors show that bands 10 and 11 make up component 2 and bands 10 and 12 make up component 5 (Table 3.1). 


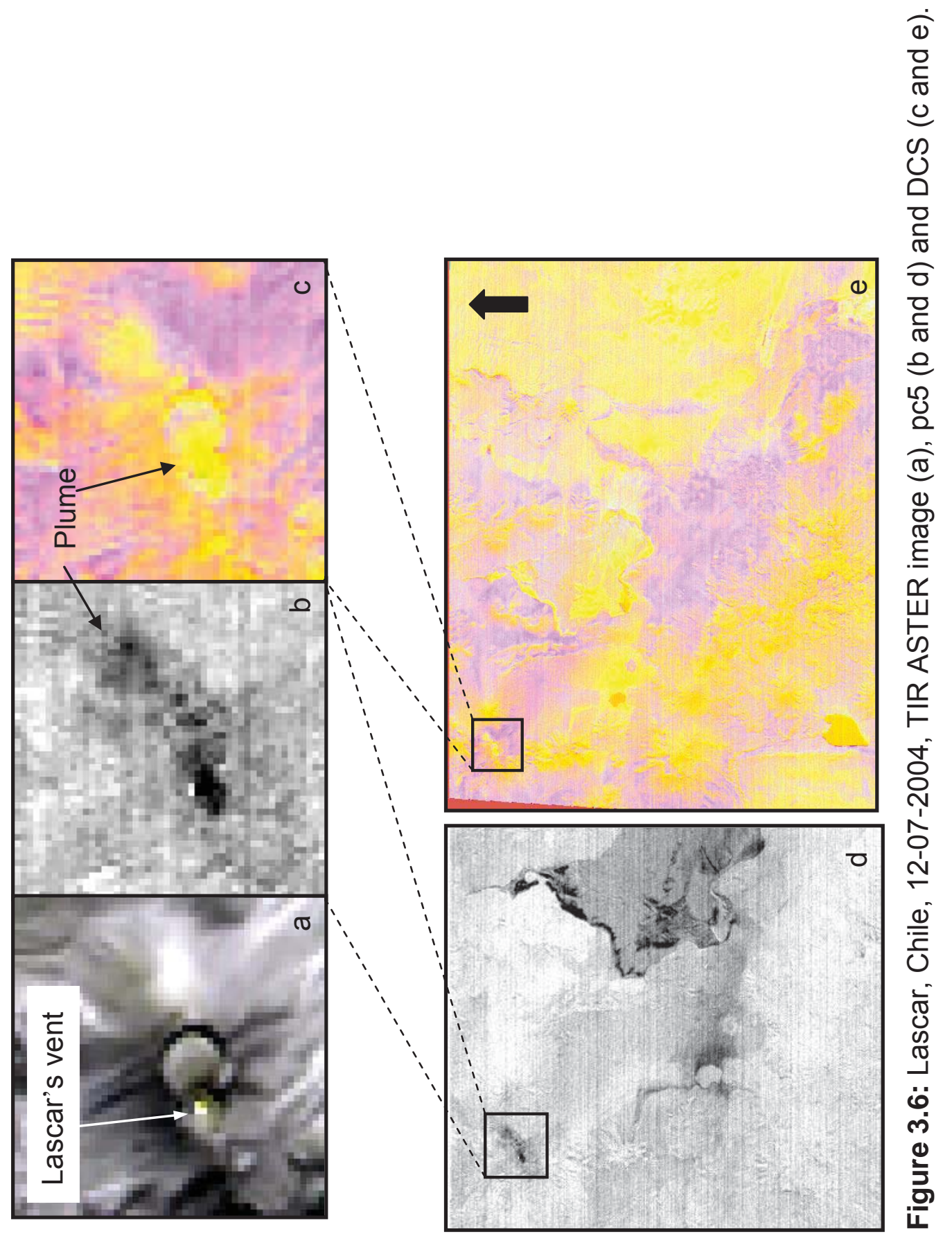




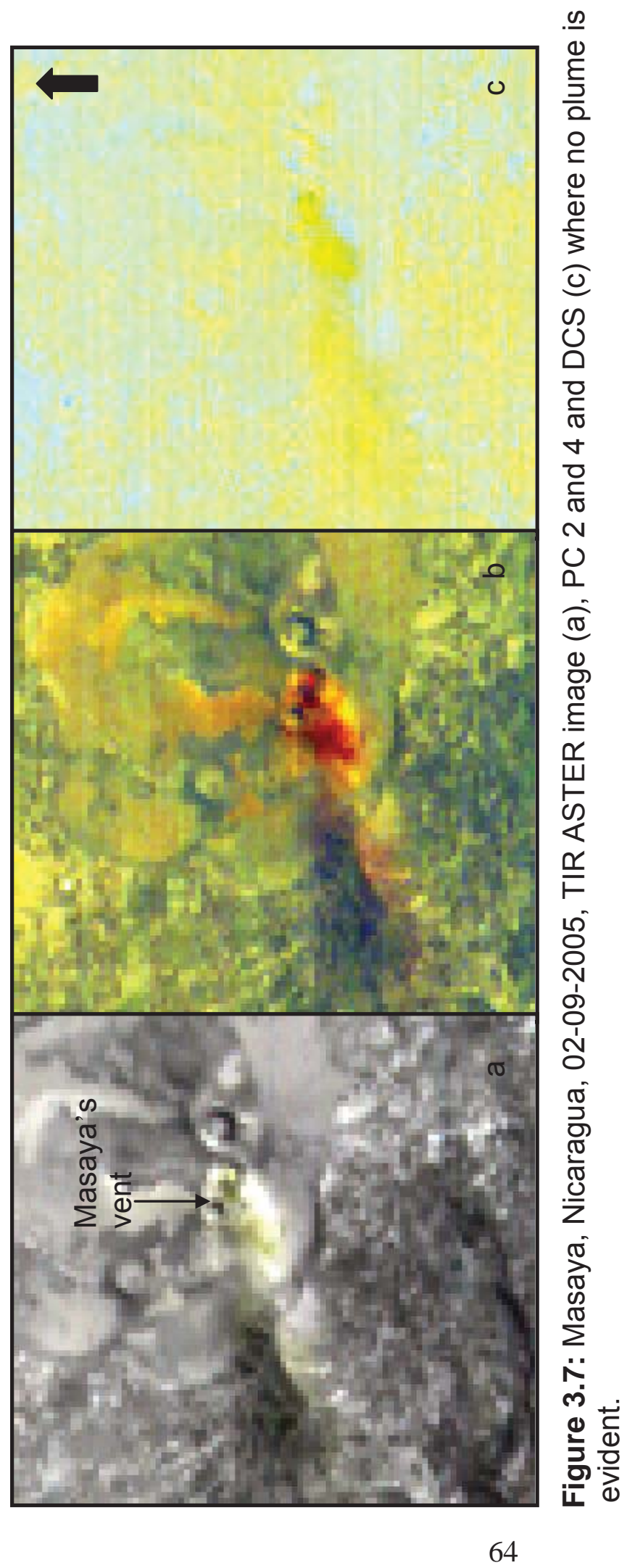




\subsection{Discussion}

The PCA and DCS analyses provided interesting results with the PCA providing slightly better interpretability than the DCS depending on the type of plume being viewed and enhanced, the composition of the plume, altitude, $\mathrm{SO}_{2}$ concentration and meteorological clouds in the image.

Plumes from explosive eruptions are easier to see in both enhancements. These plumes are typically composed of ash and gases such as $\mathrm{SO}_{2}$ and water vapor. These plumes are also typically larger in size and found at higher altitudes in the atmosphere than those from passively degassing sources. Chaiten and Eyjallajokull produced several images where the PCA and DCS enhancements consistently highlight the plume as well as indicate the different constituents of the plumes such as ash. The plume from each volcano during their eruptive episodes is visible in the TIR image. However, the enhancements of the plume from Chaiten on 31 May 2008 allow the extent of the plume to be seen more clearly (Figure 3.1). From the TIR image, the plume appears to be dominantly moving in an eastwards direction, while the enhancements show the plume also extends in a south east direction. The portion of the plume moving southeast appears to be mostly gas in composition whereas the ash is moving to the east. The DCS enhancement suggests that this gas is water vapor (blue in color). The DCS also illustrates that the portion of the plume moving eastward is silicate ash (orange/red in color) but $\mathrm{SO}_{2}$ (yellow in color) is seen around the edge of the plume. The PCA shows the varying constituents of the plume. However, unlike the DCS, there is no consistency with the colors produced for these components. The color may be different for each principal component analysis for different images of different areas due to the transformation being scene dependent. The colors in the DCS are consistent 
because the image is being normalized back to the original viewing axes (refer to methodology 3.2) so the color is standardized to an 8 bit image (Alley 1996).

It is with passively degassing plumes, composed of $\mathrm{SO}_{2}$ and found at lower altitudes, where the transformation had difficulty identifying the plumes from the images, especially the DCS. Large plumes from volcanoes such as Ubinas, Popocatepetl and Turrialba (Figures 3.3, 3.4 and 3.5 respectively) have a mixed result with the PCA being excellent at highlighting the plumes from the ASTER images. The DCS picks out the plumes, but the plume boundaries are not well defined at Ubinas and Turrialba. The plumes are yellow in color indicating they are primarily $\mathrm{SO}_{2}$ in composition, but the rest of the image is also this color making it difficult to outline the plume immediately. This problem with being unable to identify the plume from the DCS enhancement is also evident with low lying plumes, or weaker plumes such as those at Masaya and Lascar (Figures 3.6 and 3.7). The DCS of these plumes does not highlight the plumes from the background and the entire image is the same color (yellow).

The DCS algorithm works most efficiently if the data being enhanced is Gaussian in distribution. When the image is bimodal or multimodal the DCS is weakened and the resulting image has less diversity in color (Alley et al. 1996). The distribution of data for the Eyjallajokull plume on 5 May 2010 is Gaussian in nature and the resulting DCS is excellent (Figure 3.8a). The distribution for Lascar on 7 December 2004 is bimodal (Figure 3.8b) and may explain why the image is primarily of a pale yellow color making it impossible to identify a plume. The distribution of the image of Ubinas is also Gaussian (Figure 3.8c), yet the plume is not easily identified and the majority of the image is the same shade of yellow. 


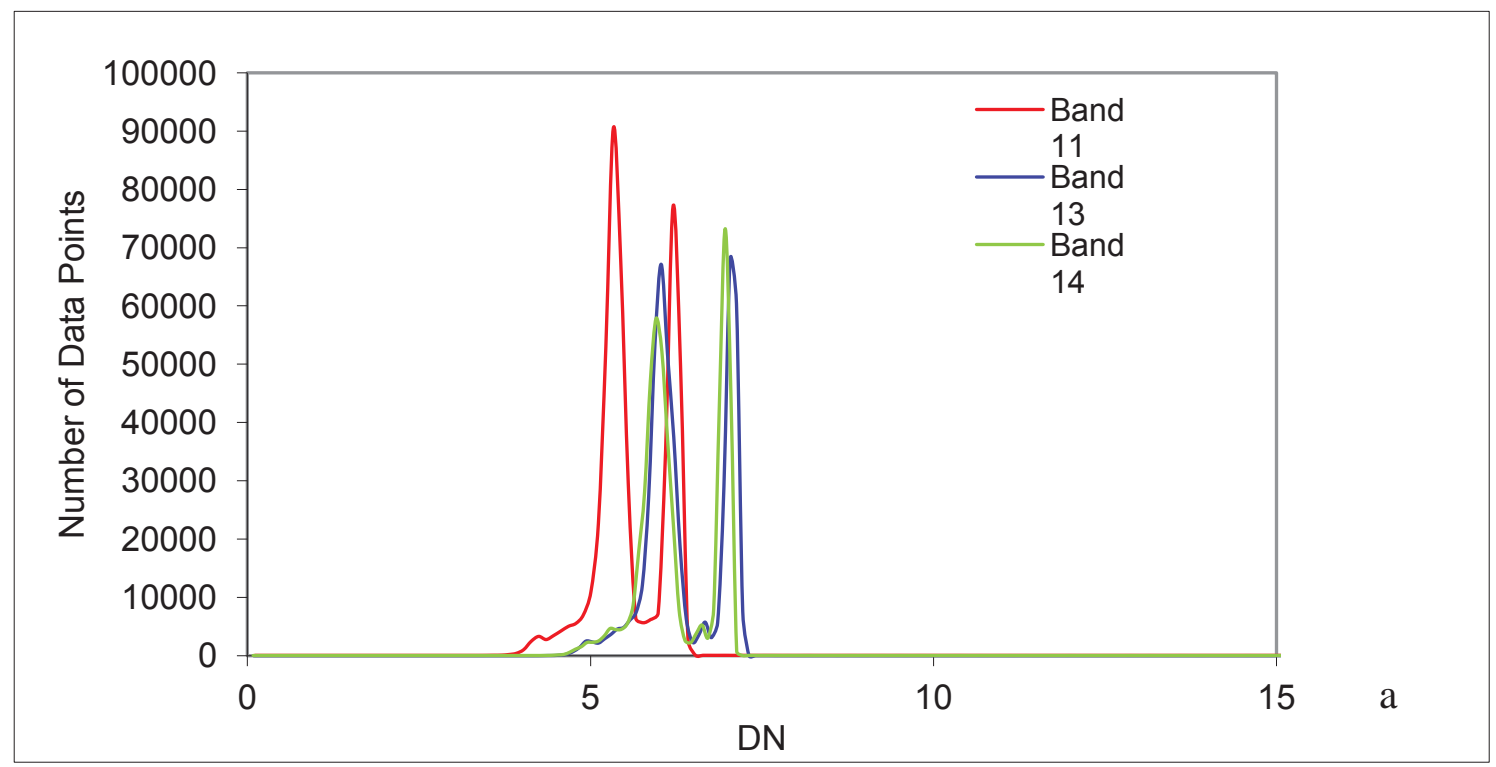

Figure 3.8 a: Data distribution for the ASTER TIR image of Chaiten, 5-31-2008.

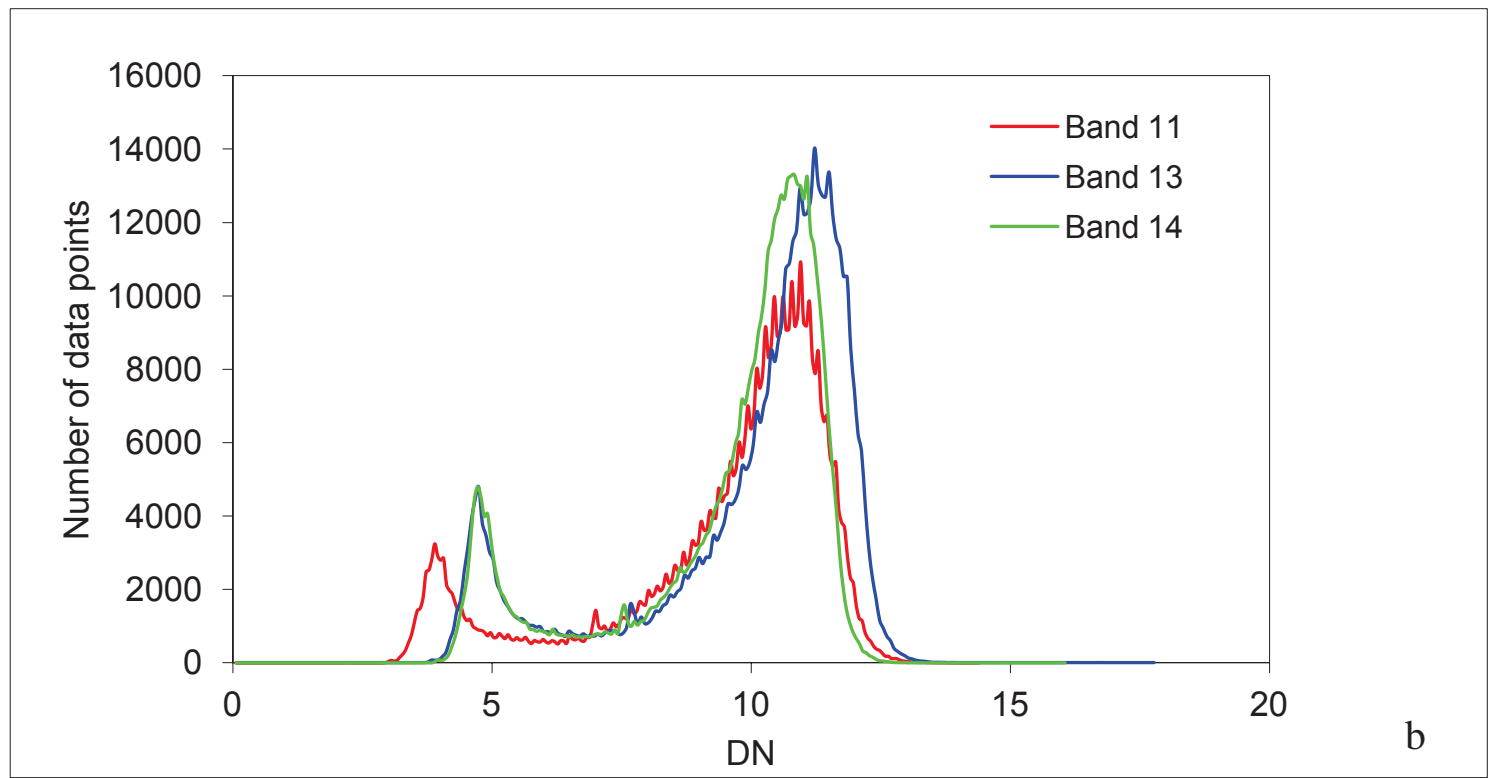

Figure 3.8 b: Data distribution for the ASTER TIR image of Lascar, 12-072004. 


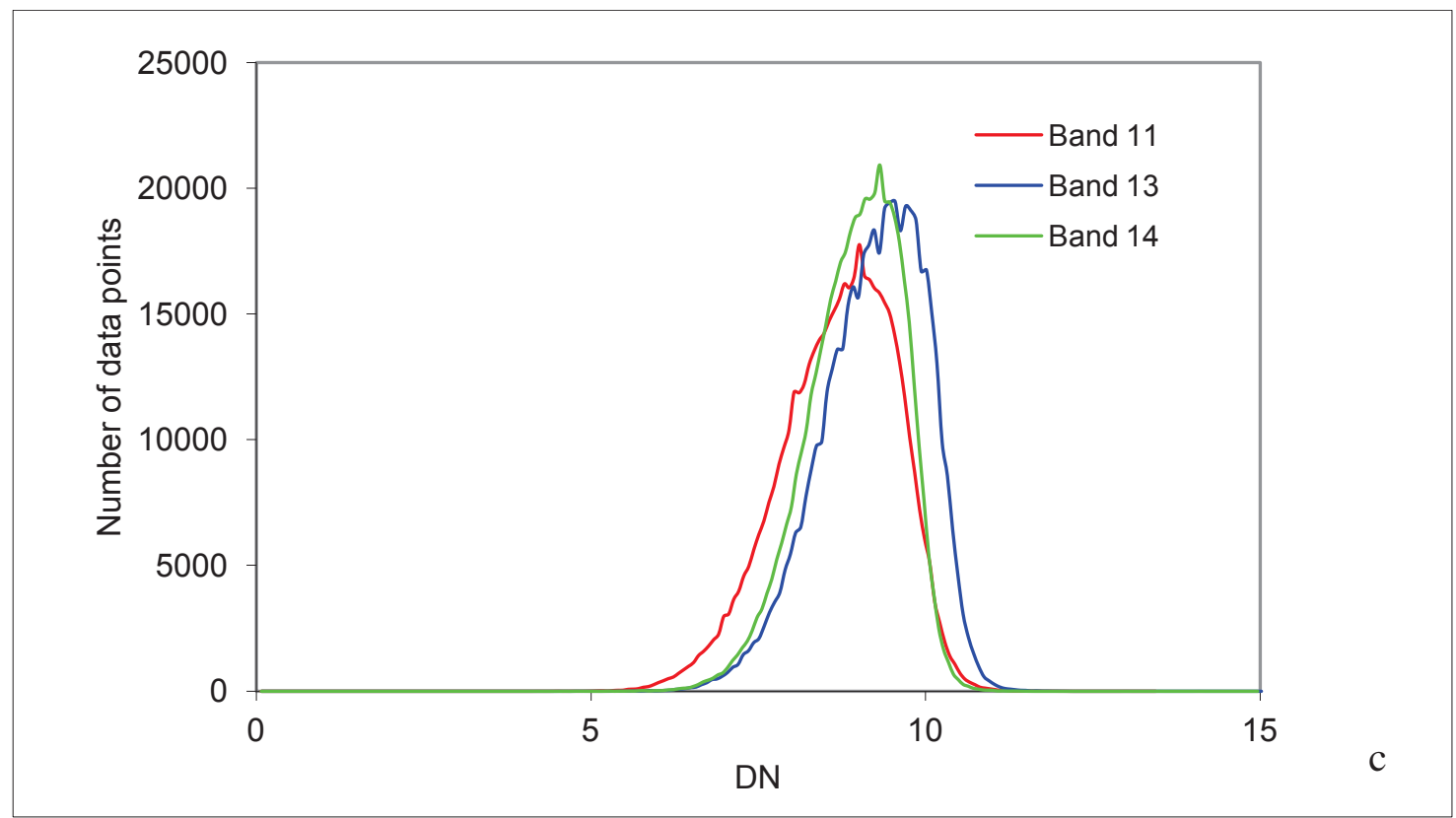

Figure 3.8 c: Data distribution for the ASTER TIR image of Ubinas, 05-082006.

The PCA transformed images for low lying plumes or weaker plumes often are better than the DCS enhancement at discriminating plumes in ASTER scenes. At Lascar the plume was weak (low $\mathrm{SO}_{2}$ concentration) and this is apparent by the fact that the ground is visible through the plume in the TIR image. Using the PCA, the plume is seen in PC 5 , which typically contains noise. The analysis may have misidentified the weak plume as noise and classified it as such. Thus, plumes with low $\mathrm{SO}_{2}$ concentrations are difficult for the DCS enhancement to pick out. At higher altitudes or with larger sized plumes such as that at Popocatepetl, where the plume is also weak and transparent, it is not impossible to identify the plume using the DCS. At Masaya, the plume is low and close to the ground. Using the PCA, a plume can be picked out from the image whereas the DCS fails. The plume is visible in 2 components, PC 2 and 
5. While the plume is not immediately obvious, it is identifiable and more evident than in the DCS.

Previously the use of the PCA has been for distinguishing ash clouds from meteorological clouds using MODIS (Hillger et al. 2002), GOES (Ellrod et al. 2003 and Ellrod 2004) and AVHRR (Dean 1994). Using the PCA and DCS analysis at Chaiten and Eyjafjallokull, ash is differentiated from other components such as $\mathrm{SO}_{2}$ and $\mathrm{H}_{2} \mathrm{O}$ (Figures 3.1 and 3.2). At Popocatepetl, the meteorological clouds over the vent and plume are easily distinguished from the plume itself in the PCA (Figure 3.4). In the DCS, the meteorological clouds are clearly not part of the plume but are not easily identified as clouds from the background as they are both an orange hue. The image of Turrialba (Figure 3.5 ) is extremely cloudy and the plume is not easily identified in the PCA or DCS from the background. The plume is only detectable because it is at a higher altitude than the clouds. If it were below the clouds it would have never been visible. As illustrated in the 28 March 2008 Hawaii scene (Figure 3.9), the PCA excels at distinguishing $\mathrm{SO}_{2}$ plumes from meteorological clouds. The color difference in the composite of components 2,3 , and 4 indicate the factor loadings contain data from bands 10,11 , and 12 . The meteorological clouds and the plume are also easily identified from one another in the DCS, even though they are the same color. This is possible because the two features do not overlap one another and the plume is a distinct plume like shape. 


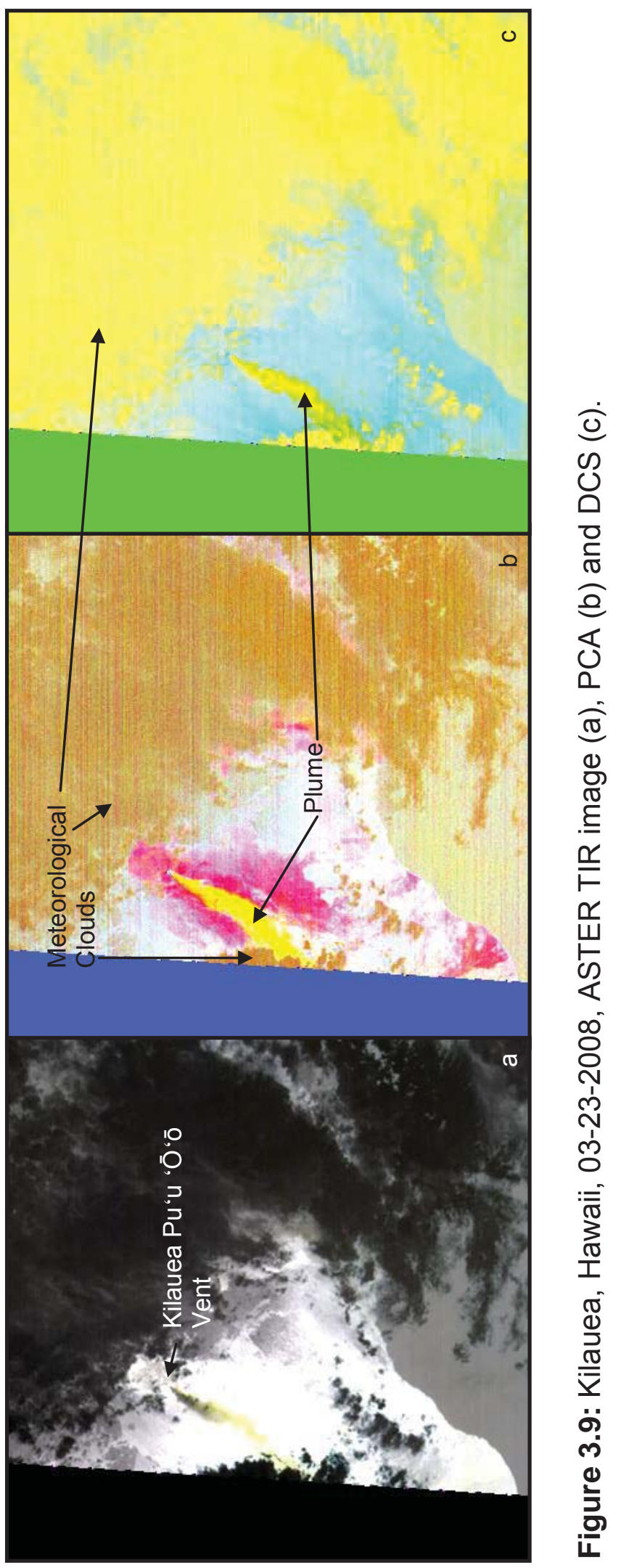




\subsection{Conclusions}

Both the PCA and DCS enhancements have advantages and disadvantages when identifying volcanic plumes. Large plumes produced from explosive eruptions can be identified from ASTER images using both techniques. However, the DCS allows identification of the varying plume components straightforwardly due to a consistent output: $\mathrm{SO}_{2}$ represented in yellow; ash in orange to pink and $\mathrm{H}_{2} \mathrm{O}$ vapor in blue. Comparing large plumes from passively degassing volcanoes, the PCA generally produced more easily identified plumes than the DCS. The PCA was not always better than the DCS when analyzing large $\mathrm{SO}_{2}$ plumes. Such an example was Turrialba where both enhancements had difficulty identifying the plume from the image. Plumes from small and low lying emitting volcanoes tested the capability of both enhancements. The PCA was able to identify the plume in every case investigated while the DCS could not identify a plume from any of the images. This is significant for this research as a large number of the volcanoes investigated are small or low lying emitters of $\mathrm{SO}_{2}$. It is important that these plumes be identified from the ASTER images using a reliable enhancement technique. Where meteorological clouds are concerned, the PCA can distinguish the plume whereas the DCS has some difficulty, depending on the scene in question.

Overall, the PCA performs better than the DCS when identifying plumes from an ASTER image. The DCS is most useful for identifying the composition of a plume, a critical step to quantifying $\mathrm{SO}_{2}$ using algorithms such as ash-sensitive MAP_SO2. 


\section{Chapter 4}

\section{An investigation into the activity at Lascar volcano during 2000-2011, using the Advanced Spaceborne Thermal Emission and Reflection Radiometer (ASTER)}




\subsection{Introduction}

Activity at Lascar has displayed cyclic behavior consisting of the growth and subsidence of a lava dome and extensive degassing from fumaroles, which is punctuated by explosive eruptions. Activity between 1984 and the late 90's has been intermittently studied through field and satellite observations (Francis and Rothery 1987; Oppenheimer et al. 1993; Matthews et al. 1997; Wooster 2001). Lascar's high altitude, arid desert environment, lack of meteorological clouds, vegetation, and snow cover make it ideal for satellite based remote sensing studies (Murphy et al. 2011). Persistent degassing from Lascar has continued up to 2007 with intermittent explosions (BGVN 2000-2007). Previous measurements of $\mathrm{SO}_{2}$ were made with a COSPEC in November 1989 and produced an average flux of $2300 \pm 1120 \mathrm{t} \mathrm{d}^{-1}$ (Andres et al. 1991). Fluxes during this time period ranged from 540 to $6970 \mathrm{t} \mathrm{d}^{-1}$. More recent measurements were made by Mather et al. (2004), with a flux of $2300 \mathrm{t} \mathrm{d}^{-1}$ in January of 2003 and Rodriguez et al. (in prep.), with a flux of around $500 \mathrm{t} \mathrm{d}^{-1}$ in December of 2004. Both these measurements were obtained using Differential Optical Absorption Spectroscopy (DOAS).

The majority of previous work carried out at Lascar has focused on the thermal anomalies and seismic activity and includes that by Francis and Rothery (1987), where a volcanic hotspot was first identified and assumed to be a lava lake before visual observations at the summit were made. Activity during the late 80's and early 90's was documented by Oppenheimer et al. (1993) and Wooster and Rothery (1997) through thermal monitoring of the volcanic dome in relation to eruptions and cyclic behavior.

With Lascar's remote location it is difficult to obtain continuous ground based gas measurements. Previous observations at the volcano have relied heavily on 
satellite images to make conclusions about activity. Tracking changes in gas emissions regularly using satellite sensors has become easier due to advancing technology and the launch of sensors such as the Advanced Spaceborne Thermal Emission and Reflection Radiometer (ASTER). ASTER has an increased spatial and spectral resolution, compared to older sensors such as the Total Ozone Monitoring Spectrometer (TOMS), resulting in a greater sensitivity to $\mathrm{SO}_{2}$. TOMS was only capable of detecting large emissions of $\mathrm{SO}_{2}$ typically during eruptive episodes such as the April 1993 eruption which released $0.4 \mathrm{Tg}$ (Bluth et al. 1997). More recently Murphy et al. (2011) investigated Lascar's thermal anomaly using ASTER's TIR bands from 2000 to 2009. As with previous studies, gas emissions were not quantified and only inferred through other observations, such as visual and seismic data. Gas data are the missing link in the observations made at Lascar and is the key to gaining a clear understanding of Lascar's volcanic system. Degassing is a fundamental part of volcanism. Quantifying emissions is important in terms of understanding the magma chamber evolution and eruption dynamics of the volcano (Sparks 1997).

This study focuses on activity at Lascar volcano from 2000-2011 utilizing ASTER data. The major goal of this study is to quantify $\mathrm{SO}_{2}$ emissions at Lascar. The resulting $\mathrm{SO}_{2}$ time series is used to identify any patterns of activity at Lascar in relation to eruptions or cyclic behavior and provide insight into the processes occurring at Lascar. In addition to the $\mathrm{SO}_{2}$ data, ASTER imagery is also used to calculate the maximum temperature of the crater hotspot at Lascar and how it changes over time in relation to the gas emissions and eruptions. Both these data sets will support monitoring efforts at the volcano and allow us to draw conclusions about future activity. 


\subsubsection{Lascar Volcano and Geological History}

Lascar Volcano, a calc-alkaline andesitic stratovolcano $\left(23.37^{\circ} \mathrm{S}, 67.73^{\circ} \mathrm{N}\right)$, is located in the central Andes of Northern Chile at an altitude of $5592 \mathrm{~m}$ (Figure 4.1a and b) (Matthews et al. 1997). Historical records date activity back to 1848 (Gardeweg et al. 1994) and the age of the volcano is thought to be less than 50 ka (Matthews et al. 1997). The history of Lascar will be only briefly described here, but for more detail, refer to Gardeweg et al. (1998).

Past activity at Lascar has been broken into 4 evolutionary stages which have resulted in the present edifice that is east-west trending (Matthews et al. 1997; Gardeweg et al. 1998) (Figure 4.1b). The first stage started with the building of Lascar's edifice, which erupted lavas of a pyroxene-andesite composition. This initial activity was then followed by 2 pyroclastic flows that produced the Saltar and Chaile andesitic flow deposits (Gardeweg et al. 1998). Stage 2 activity started with activity moving westward. Deposits during this stage indicate that a sub-glacial eruption occurred. The deposits also indicated the presence of a large dome complex. The largest eruption recorded at Lascar was during this second stage and is known as the Soncor eruption. This eruption produced 10$15 \mathrm{~km}^{3}$ of pyroclastic flow and plinian fallout deposits and was dated at $26.45 \mathrm{ka}$ (Matthews et al. 1997; Gardeweg et al. 1998). A stratocone was built during stage 3 over the site of the Soncor eruption and ended in an andesitic explosive eruption with deposits dated at 9.2 ka (Tumbres deposit) (Matthews et al. 1997). The fourth stage produced thin pyroclastic flow layers and the TumbresTalabre lava that is dated at $7.1 \mathrm{ka}$ (Matthews et al. 1997). Activity during this final stage resulted in an eastward shift to what is presently the active area of the volcano. Multiple crater collapses occurred truncating the lava flow and indicating a westward movement of activity. It is the deepest of these craters that is the presently active crater (Figure 4.1). It is believed that these craters 
were a result of degassing within the shallow magma reservoir. Figure 4.2 is a geological map of the volcanic products produced from each of the 4 stages of activity discussed here.

Matthews et al. (1994b) have closely examined the petrology and geochemistry of Lascar's volcanic rocks to determine what has previously occurred within the magma chamber. They proposed that the magma chamber at Lascar was continuously fractionating and periodically receiving new batches of magma with a basaltic andesite composition. This basaltic andesite mixed with the older more evolved magma in the chamber to produce the deposits seen today that are primarily of an andesitic composition. Matthews et al. (1994b) also indicate that the $\mathrm{SO}_{2}$ emissions are accounted for by the oxidation and quenching of sulfide rich mafic magma when it mixes with older more evolved magma in the chamber. 


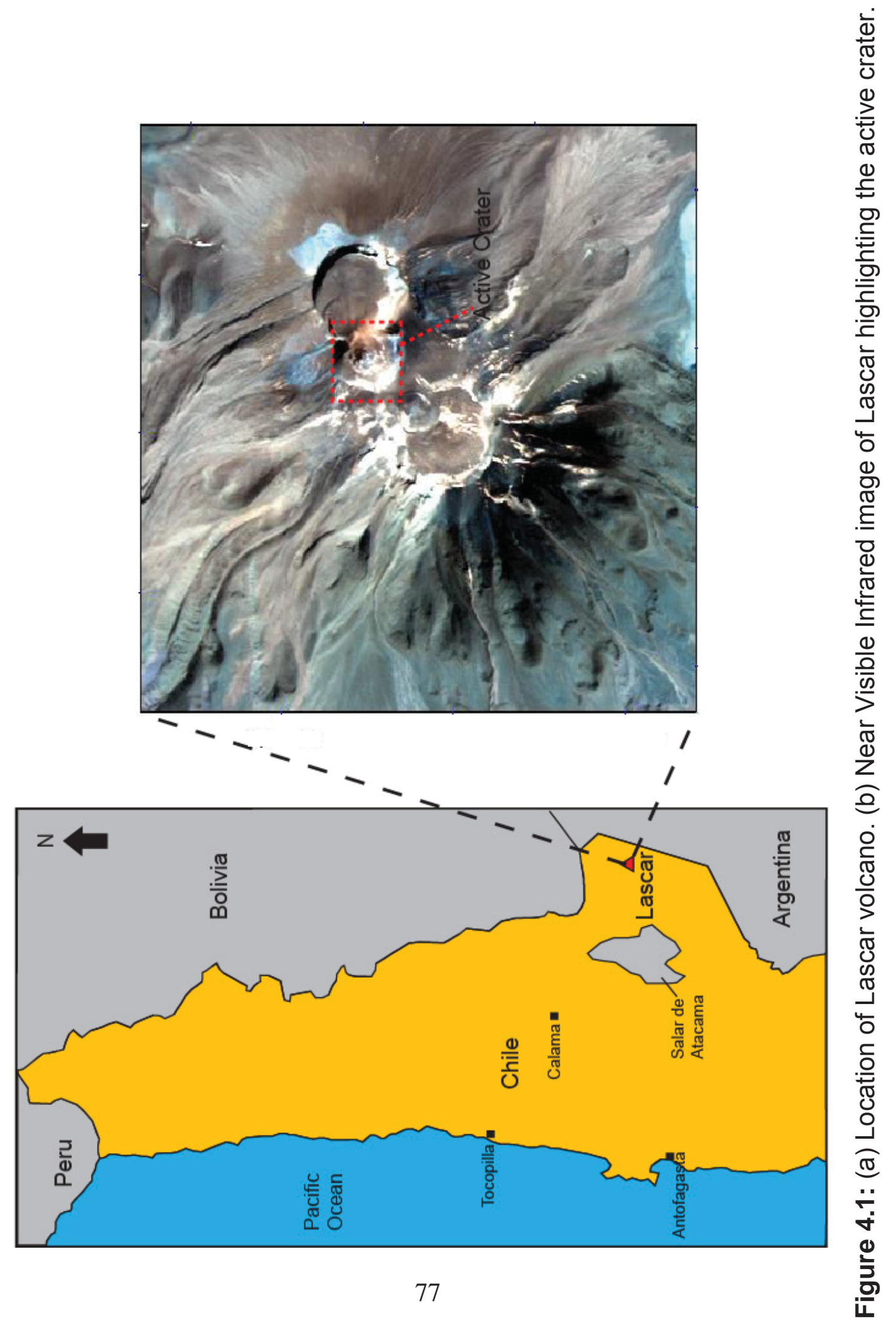




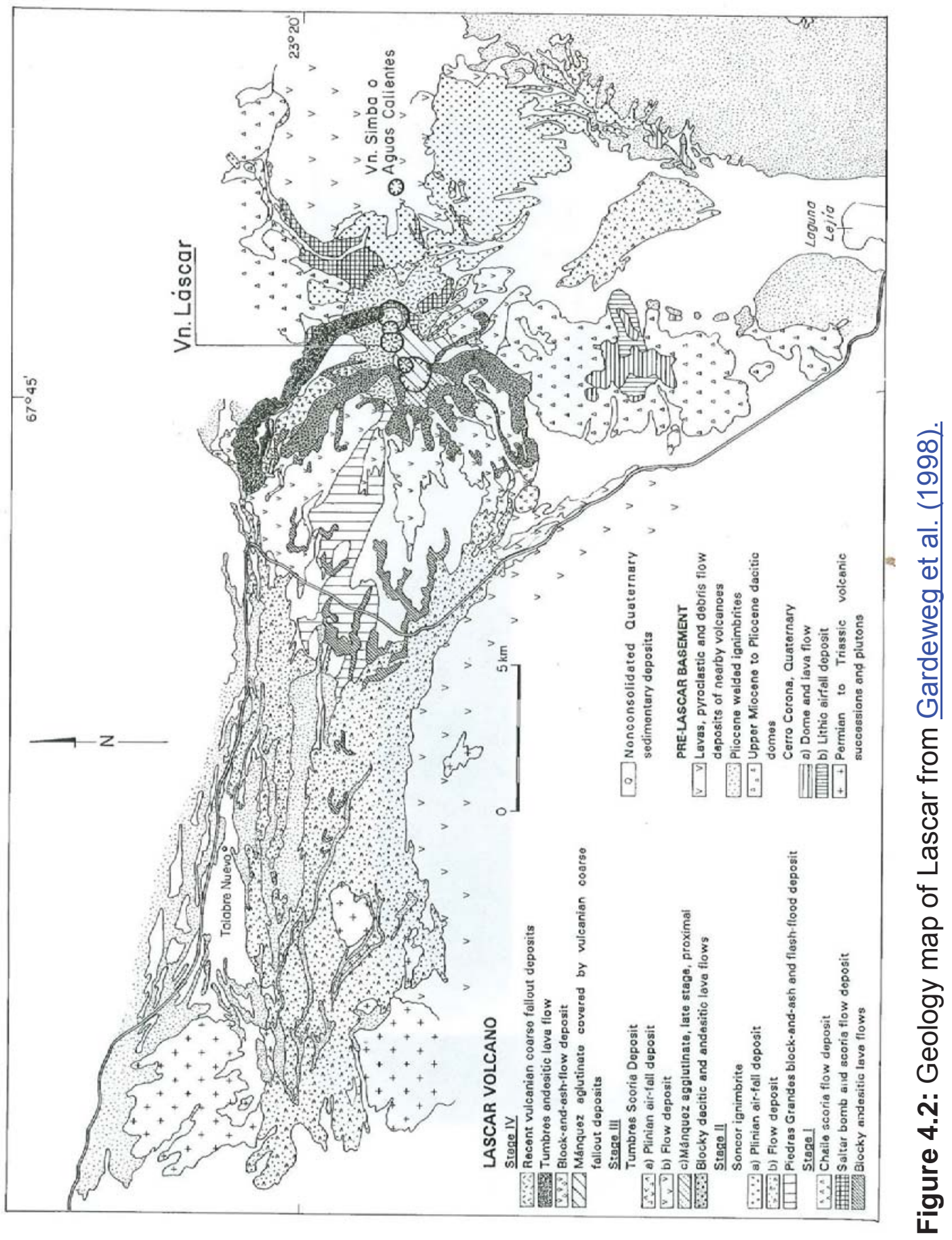




\subsubsection{4-1993 Cyclic Activity}

Recent activity displayed cyclic behavior with 4 distinctive cycles occurring between 1984 and 1993 (Matthews et al. 1997). Each cycle typically consisted of a lava dome being extruded from the crater. Vigorous degassing from high temperature fumaroles occurred on and around the dome and produced a constant plume above the volcano. The dome then subsided back into the crater and conduit. During this period of subsidence, fumarolic gas emissions declined. The end of the cycle concluded with a violent eruption. Throughout the cycle many minor eruptions also occur. Matthews et al. (1997) extensively described and discussed each cycle through visual and satellite observations.

\section{Cycle 1: 1984-September 1986}

Activity at Lascar was first noticed in 1984 after a hot spot was detected on a Landsat TM image (Rothery et al. 1988; Glaze et al. 1989 a; b; Oppenheimer et al. 1993). This thermal anomaly was due to the presence of an active lava dome in the crater and the emission of hot gases from fumaroles dispersed on and around the dome. From 1984 to 1986 the thermal anomaly diminished in size until a powerful eruption occurred in September 1986. The eruption reached an altitude of $10 \mathrm{~km}$ above the crater (Matthews et al. 1997). It is believed that the decrease in the thermal anomaly was because the dome was subsiding into the conduit, preceding the eruption. After the eruption very little activity occurred at Lascar up to the end of 1988.

\section{Cycle 2: February 1989-February 1990}

In February of 1989 a new lava dome was seen growing in the active crater at Lascar (BGVN 1989). Observations indicated that by October the dome had already started to subside. $\mathrm{SO}_{2}$ recorded during November was detected by COSPEC with fluxes of $2300 \pm 1120 \mathrm{t} \mathrm{d}^{-1}$ (Andres et al. 1991). Steam eruptions 
occurred throughout December and thermal activity detected through satellite imagery had dropped to a very low level (Oppenheimer et al. 1993). This cycle was concluded with 3 strong explosions on 20 February 1990 producing eruption columns 8-14 km above the crater (Matthews et al. 1997). Surveillance of the crater in March 1990 revealed that the dome had almost disappeared.

\section{Cycle 3: February 1992-April 1993}

A new dome was evident in February/March 1992 and coincided with a peak in thermal radiance (Oppenheimer et al. 1993). High temperature fumaroles were also apparent. Dome subsidence was not observed until November 1992. Wooster and Rothery (1997) suggested that subsidence occurred well before November, based on a decreasing thermal anomaly detected by satellite imagery. The dome had completely disappeared by March 1993. Many minor eruptions were recorded during the subsidence phase of the cycle, which culminated in a strong eruption on 19 April. The eruption column was sustained for 2 days (19-20 April 1993), and ranged in height between 5 and $25 \mathrm{~km}$ before collapsing to produce pyroclastic flows. An estimated $0.1 \mathrm{~km}^{3}$ of magma was erupted and 400 kilotons of $\mathrm{SO}_{2}$ were produced (BGVN 1993).

\section{Cycle 4: April 1993-December 1993}

After the end of Cycle 3 and the 19 April 1993 eruption, a new lava dome was observed by the end of the month. During August, a small explosion occurred and the dome had started to subside (BGVN 1993). On the dome itself, a depression formed, reaching a depth of $100 \mathrm{~m}$ by November. Thermal radiance dropped to $0^{\circ} \mathrm{C}$ by December, indicating that dome activity and degassing were low. This cycle culminated with in eruption in December 1993 producing an eruption column $10 \mathrm{~km}$ high (BGVN 1994). 
Matthews et al. (1997) developed a model to explain the cycles of activity at Lascar. The eruptions are associated with the subsidence of the dome after it grows. The dome subsides as a result of partial degassing of the magma. During the formation of the dome, the magma in the conduit and interior of the dome becomes vesicular and bubbly. As this process continues deformation occurs and the bubbles become sheared and start to coalesce (Cashman and Mangan 1994). The shearing of these bubbles increases the permeability of the magma making it easier for gases to escape from the magma through the dome. Eventually, the weight of the dome exceeds the strength of the bubbly foam-like magma layer and the bubbles collapse resulting in dome subsidence. Permeability of the magma decreases and pressure builds up as trapped gases exsolve in a closed system. When the overpressure reaches a threshold an explosive eruption takes place (Cashman and Mangan 1994).

Post - 1993 activity

Activity since the end of 1993 has been less coherent and cyclic behavior has not been identified. The dome from the December 1993 eruption had completely disappeared by February 1994 and was replaced by a deep hole (Mathews et al. 1997). Throughout 1994, 1995, and 1996 there was persistent degassing punctuated by small explosions reaching 2-3 km above the crater (BVGN 1994-1996). These explosions are thought to be a result of the crater floor becoming blocked, allowing pressure to build up.

On 20 July 2000, an eruption took place at Lascar producing a plume that reached altitudes of 10.7-13. $7 \mathrm{~km}$ (BGVN 2000). Minor eruptions occurred in October 2002 (BVGN 2003). In January 2003, $\mathrm{SO}_{2}$ was measured using the mini UV DOAS and produced a flux of $2300 \mathrm{t} \mathrm{d}^{-1}$ (Mather et al. 2004). On 4 May 2005 , an eruption occurred sending a plume $10-11 \mathrm{~km}$ in to the atmosphere 
(BGVN 2005). After this eruption, 3 new fumaroles were evident around the crater. $\mathrm{SO}_{2}$ was measured by Rodriguez et al. (In prep) in December 2004 and emissions of $\sim 500 \mathrm{t} \mathrm{d}^{-1}$ were recorded using the mini UV DOAS.

On the 18 April 2004, 4 eruptions occurred with the largest reaching an altitude of $10-15 \mathrm{~km}$ above the vent (BVGN 2006). Explosions continued for another 5 days. On 4 May 2005 a large eruption occurred at Lascar producing an ash cloud $10 \mathrm{~km}$ above the crater (BGVN 2005). Ash fell up to $1000 \mathrm{~km}$ away from the volcano and there were no visual reports of the eruption which was observed by satellite imagery (BGVN 2005). In late 2006 and early 2007, Lascar continued to emit ash with the last reported eruption from the BGVN indicating that an ash plume rose $6.7 \mathrm{~km}$ high above the crater on the 22 January 2007(BGVN 2007).

\subsubsection{ASTER Instrument}

ASTER is a high spatial resolution, multi spectral instrument with 14 bands that cover the visible near infrared (VNIR), shortwave infrared (SWIR) and thermal infrared (TIR). The 5 TIR bands are utilized in this study to quantify $\mathrm{SO}_{2}$ and determine hot spot temperatures from Lascar volcano since 2000 until present. With a spatial resolution of $90 \mathrm{~m}$ in the TIR, ASTER is capable of detecting emissions from passively degassing volcanoes with emissions of less than $1000 \mathrm{t} \mathrm{d}^{-1}$, such as Lascar. Using a forward radiative transfer model, Henney (2006) found that ASTER is able to detect $\sim 400 \mathrm{t} \mathrm{d}^{-1} \mathrm{SO}_{2}$ in a $4 \mathrm{~km}$ altitude plume in a tropical atmosphere. Other sensors with coarser spatial resolution, for example, MODIS (1 $\mathrm{km}$ spatial resolution) might not be proficient at detecting emissions from Lascar. The TIR bands of ASTER are capable of looking at volcanoes during the night providing nighttime and daytime images.

The temporal resolution of ASTER is such that an image is obtained roughly 
every 16 days. This is not useful for real time monitoring purposes. But this resolution is beneficial when looking at a collection of images at a volcano, over an extensive period of time, to investigate behavior and identify patterns of activity that can be used as a future reference as to when a volcano may erupt. The cycles at Lascar occur over several months to over a year (Matthews et al. 1997). It is unknown if these cycles have continued since 1993. ASTER is the ideal instrument to monitor the changes in activity at Lascar volcano to determine if cyclic behavior is continuing. For a more in depth description of the ASTER instrument please refer to the Introduction (Chapter 1). 


\subsection{Methodology}

\subsubsection{ASTER MAP SO2 Algorithm}

$\mathrm{SO}_{2}$ fluxes can be calculated from Level $1 \mathrm{~b}$ ASTER TIR imagery. The $\mathrm{SO}_{2}$ absorption feature at $8.6 \mu \mathrm{m}$ is detected by ASTER bands 10,11 and 12 . The retrieval of $\mathrm{SO}_{2}$ is accomplished using an IDL code, MAP_SO2, which is built on the MODTRAN radiative transfer code (Berk et al. 1989). The retrieval of $\mathrm{SO}_{2}$ using the MAP_SO2 code is based on the at-sensor radiance expression developed by Realmuto et al. (1994):

$$
L_{s}=\left\{\varepsilon_{g} B\left(T_{g}\right)+\left(1-\varepsilon_{g}\right) L_{d}\right\} t_{a}+L_{u}
$$

where $\varepsilon_{\mathrm{g}}$ is the ground emissivity; $\mathrm{B}$, the Planck function; $\mathrm{T}_{\mathrm{g}}$, the temperature of the ground; $t_{a}$, the transmission of the atmosphere; $L_{d}$, the downwelling radiance; and $\mathrm{L}_{\mathrm{u}}$ the upwelling radiance. The MAP_SO2 code contains spectral band models of 12 atmospheric gas molecules, including $\mathrm{SO}_{2}$. These band models are used along with a user-defined atmosphere, from which upwelling $\left(L_{u}\right)$ and downwelling $\left(L_{d}\right)$ radiances are derived, and the spectral emissivity of the ground $\left(\varepsilon_{\mathrm{g}}\right)$ to calculate the radiance received at the sensor $\left(L_{s}\right)$. As none of the parameters mentioned above can be easily determined by the at-sensor radiance spectra, the retrieval of $\mathrm{SO}_{2}$ is a complex inverse problem (Realmuto et al. 1997; Realmuto 2000). Additional information is loaded into MAP_SO2, such as plume altitude and thickness to make the inverse problem less illconstrained. With this additional information only the $\mathrm{SO}_{2}$ column abundance, emissivity and the ground temperature $\left(T_{g}\right)$ need to be considered. To determine the $\mathrm{SO}_{2}$ column abundance, the 'true' ground temperature and emissivity have to be calculated using the at-sensor radiance expression (Eq. (1)) in ASTER channels 13 and 14, as they lie outside the $\mathrm{SO}_{2}$ absorption 
feature. The 'apparent' ground temperature is determined by solving the at sensor radiance expression (Eq. (1)) using channels lying within the $\mathrm{SO}_{2}$ absorption feature (channels 10,11 and 12). It is the difference between the true and apparent ground temperatures that allow the $\mathrm{SO}_{2}$ load to be calculated, as the temperature difference is a linear function of the $\mathrm{SO}_{2}$ burden (Realmuto 1994; Watson et al. 2004). The result is an $\mathrm{SO}_{2}$ map of the dimensions of the original image with the units of $\mathrm{g} \mathrm{SO}_{2}$ per $\mathrm{m}^{2}$ (i.e. column integrated $\mathrm{SO}_{2}$ burden).

Errors associated with this technique equate to a maximum of $31 \%$ (Henney 2006). Errors are primarily related to sensor calibration, sensor sensitivity and the accuracy of the MAP_SO2 retrieval such as plume height and atmospheric data. For a full description of the errors related to this method please refer to the Methodology in the introductory chapter (Chapter 1 Section 1.2.3.).

\subsubsection{ASTER Ground Temperature.}

Ground temperature was calculated using ASTER's on demand surface kinetic temperature product (AST08), using the 5 TIR channels (10-14) at $90 \mathrm{~m}$ spatial resolution, for areas of land only (Land Process Distributed Active Archive Center (LP DAAC)). Daytime and night-time images can both be processed by the kinetic temperature product to investigate crater temperature at Lascar. The kinetic temperature product is produced by applying the Temperature Emissivity Separation (TES) algorithm to atmospherically corrected ASTER surface radiance (TIR) data (AST_09T) (Gillespie et al. 1999; Ramsey and Dehn 2004). Planck's Law is then applied to the emissivity values produced from the TES algorithm (Gillespie et al. 1999; LP DAAC). The TES algorithm estimates emissivities in the TIR ASTER data by utilizing the Normalized Emissivity Method (NEM) (Realmuto 1990; Gillespie et al. 1999). The emissivity estimates 
produced are utilized to establish the land leaving radiance that is a result of sky irradiance. The figure produced is then subtracted from the TIR radiance to estimate the emitted radiance from which the temperature of the ground is calculated using the NEM module (LP DAAC). The final product is in degrees Kelvin. For more details on the TES algorithm and ASTER's surface kinetic temperature product please refer to the NASA's homepage (http:/asterweb.jpl.nasa.gov) or Gillespie et al. (1999).

Crater temperatures were determined using the simple approach of selecting the maximum temperature of the pixels within Lascar's active crater. Several other studies have used this method of taking the single maximum pixel temperature to represent the volcanoes hotspot/thermal feature such as Ramsey and Dehn (2004) and Vaughan and Hook (2006). Ramsey and Dehn (2004) used the maximum single pixel temperature to look at the dome at Bezymianny volcano, Kamchatka during an eruptive episode in 2000 with ASTER. The authors assumed that where only 1 ASTER pixel illustrated a high thermal anomaly that a sub-pixel heat source was responsible, such as an active fumarole. Vaughan and Hook (2006) also used the maximum single pixel method with ASTER data to measure changes inside the crater at Mt St Helens. They relate the changes in temperature to different phases of dome growth. This study was also important in terms of deriving what baseline thermal behavior is typical before an eruption at Mt St Helens. Both these studies use the ASTER surface kinetic temperature product and used a similar methodology to this study. 


\subsubsection{Errors}

ASTER data are advantageous because they are sensitive to volcanic features that are warm $\left(<100^{\circ} \mathrm{C}\right)$, as well as very hot volcanic features $\left(>100^{\circ} \mathrm{C}\right)$ (Vaughan et al. 2010). The temperature of a pixel is a combination of all the features contained in that pixel. The hot volcanic feature only accounts for a portion of that pixel. Hence, the temperature of the pixel is not an accurate reflection of the thermal feature. Much work has been carried out using satellite based sensors to identify temperatures of sub pixel features (Dozier 1981; Crisp and Baloga 1990; Oppenheimer et al. 1991; Wright et al. 2003). Vaughan et al. (2010) specifically investigated the use of ASTER at detecting sub-pixel thermal features. They wanted to determine how large a hot sub-pixel feature of a given temperature needs to be to resolve a thermally mixed pixel. Also, how large a hot sub-pixel feature needs to be to saturate that pixel?

Vaughan et al. (2010) found that a thermally mixed pixel, with a background temperature of $0{ }^{\circ} \mathrm{C}$, became saturated if the hot volcanic portion, with a temperature of $200^{\circ} \mathrm{C}$, occupied more than $30 \%$ of the pixel. If either the temperature difference or percentage of the pixel occupied by the hot feature is decreased then saturation will not occur. For very hot volcanic features $\left(800^{\circ} \mathrm{C}\right.$ above the background temperature) only $2-3.5 \%$ of the pixel need be occupied for saturation to occur (Vaughan et al. 2010). With the Lascar crater pixel certainly being thermally mixed, not knowing the temperature of the volcanic features, and what percentage of the pixel they occupy adds a significant error to this study (Vaughan et al. 2010). It is not known specifically what this error is; however, our interpretation of the events and activity at Lascar will be affected by this uncertainty. Previous temperatures of the dome at Lascar (prior to 1995) were estimated between $90-200^{\circ} \mathrm{C}$ (Oppenheimer et al. 1993; Wooster and Rothery 1997). 
The maximum pixel temperature of the crater will provide a guide to changes in activity at Lascar. It will not be known if we are looking at several fumaroles occupying a small portion of the pixel or a dome occupying most of the pixel. One way to tackle this problem would be to use high resolution visible imagery to view the crater at Lascar. The actual calculation of the pixel temperature used to produce the ASTER kinetic temperature product has an accuracy of 1.5 $\mathrm{K}$

(http://asterweb.jpl.nasa.gov/content/03 data/01 Data Products/release surfac e kinetic temperature.htm).

The MAP_SO2 algorithm has an error of $\sim 31 \%$ (Henney 2006; Campion et al. 2010). This error comes from a variety of sources including system calibration, and the sensitivity and accuracy of the $\mathrm{SO}_{2}$ retrieval (refer to Chapter 1 section 1.2.3.). The errors with ASTER and the MAP_SO2 retrieval are well understood. 


\subsection{Results}

\subsection{1. $\mathrm{SO}_{2}$ at Lascar Volcano}

Out of the 200 ASTER images obtained for this study 26 were useable for quantification of $\mathrm{SO}_{2}$ emissions from Lascar. The other 174 ASTER images were either too cloudy, no $\mathrm{SO}_{2}$ was apparent, or the temperature contrast between the ground and plume was zero meaning it was unable to be quantified. Using a TIR channel combination of $14(\mathrm{R}), 13(\mathrm{G})$ and $11(\mathrm{~B})$ to view the images in RSI ENVI software, any $\mathrm{SO}_{2}$ present in the images is seen as a yellow color, typically in the active crater at Lascar. Images where this yellow color could be seen in and around the crater were processed further with the MAP_SO2 algorithm. Principal Components Analysis (PCA) and Decorrelation Stretch (DCS) enhancements were carried out on the TIR images to highlight the plumes and their extent in the images (refer to Chapter 3 for specific details on the PCA and DCS enhancements). The DCS did not highlight any of the $\mathrm{SO}_{2}$ plumes at Lascar. Of the 26 images where $\mathrm{SO}_{2}$ could be identified only 15 produced $\mathrm{SO}_{2}$ maps where a plume was evident and a flux could be quantified. These images along with the PCA enhancement and $\mathrm{SO}_{2}$ maps are illustrated in Table 4.1. The location of the transects is shown on the $\mathrm{SO}_{2}$ maps and information on the wind speed and direction is provided.

It can be seen that the plumes all typically flow from the vent in a westerly or south westerly direction. In all the images, the strongest concentration of $\mathrm{SO}_{2}$ is found in the crater which is where the fumaroles are expected to be located. In many of the $\mathrm{SO}_{2}$ maps the plumes are extensive and puffing is evident (e.g. 2 October 2000). Yet, in several of the images plumes are not very obvious. Such an example is the 9 June 2000 image. In this image the crater is inundated with $\mathrm{SO}_{2}$ and the plume is barely leaving the crater. The flux for this image (2372 $\mathrm{t} \mathrm{d}^{-}$ 
${ }^{1}$ ) is much higher than other plumes where the plume is extensive, such as the plumes imaged on 2 October 2000 and 9 October 2000.

The other 11 images produced $\mathrm{SO}_{2}$ maps where no plume was evident, but the crater was inundated with $\mathrm{SO}_{2}$. Table 4.2 shows the TIR images and MAP_SO2 maps of the 11 dates where no $\mathrm{SO}_{2}$ flux was determined. To determine the $\mathrm{SO}_{2}$ flux of a plume the wind speed is needed. With no $\mathrm{SO}_{2}$ flowing out of the crater forming a plume, it is assumed that the wind speed is zero. With no wind speed, the gas flux cannot be established. We cannot be certain that a plume was present at Lascar when these images were acquired. If any $\mathrm{SO}_{2}$ has escaped from the crater forming a plume its column abundance may be too low to be detected by ASTER. Other possible reasons why no plume was imaged include a low thermal contrast between the plume and the ground, making the retrieval unable to detect $\mathrm{SO}_{2}$, or a high wind speed. It is also quite possible that the $\mathrm{SO}_{2}$ in these images might be an artifact or that the $\mathrm{SO}_{2}$ has falsely been detected. Other images show areas where $\mathrm{SO}_{2}$ has been falsely detected such as the 14 March 2000 and 24 April 2011 images.

Radiosonde data acquired in Antofagasta, Chile, were used to determine wind speed in this study. Antofagasta is over 100 miles away from Lascar; therefore the wind speed is not accurate. The wind direction recorded from the radiosonde profiles is not consistent with the plume direction seen in the MAP_SO2 images. This again emphasizes the inaccuracy of using the radiosonde data. The gas must dissipate from the crater somehow. If it is not forming a plume it is either lost to the atmosphere through conversion to sulfate aerosols or dry deposition. Sulfate aerosols can affect the MAP_SO2 retrieval making it difficult for ASTER to detect $\mathrm{SO}_{2}$ (Campion et al. 2010). 


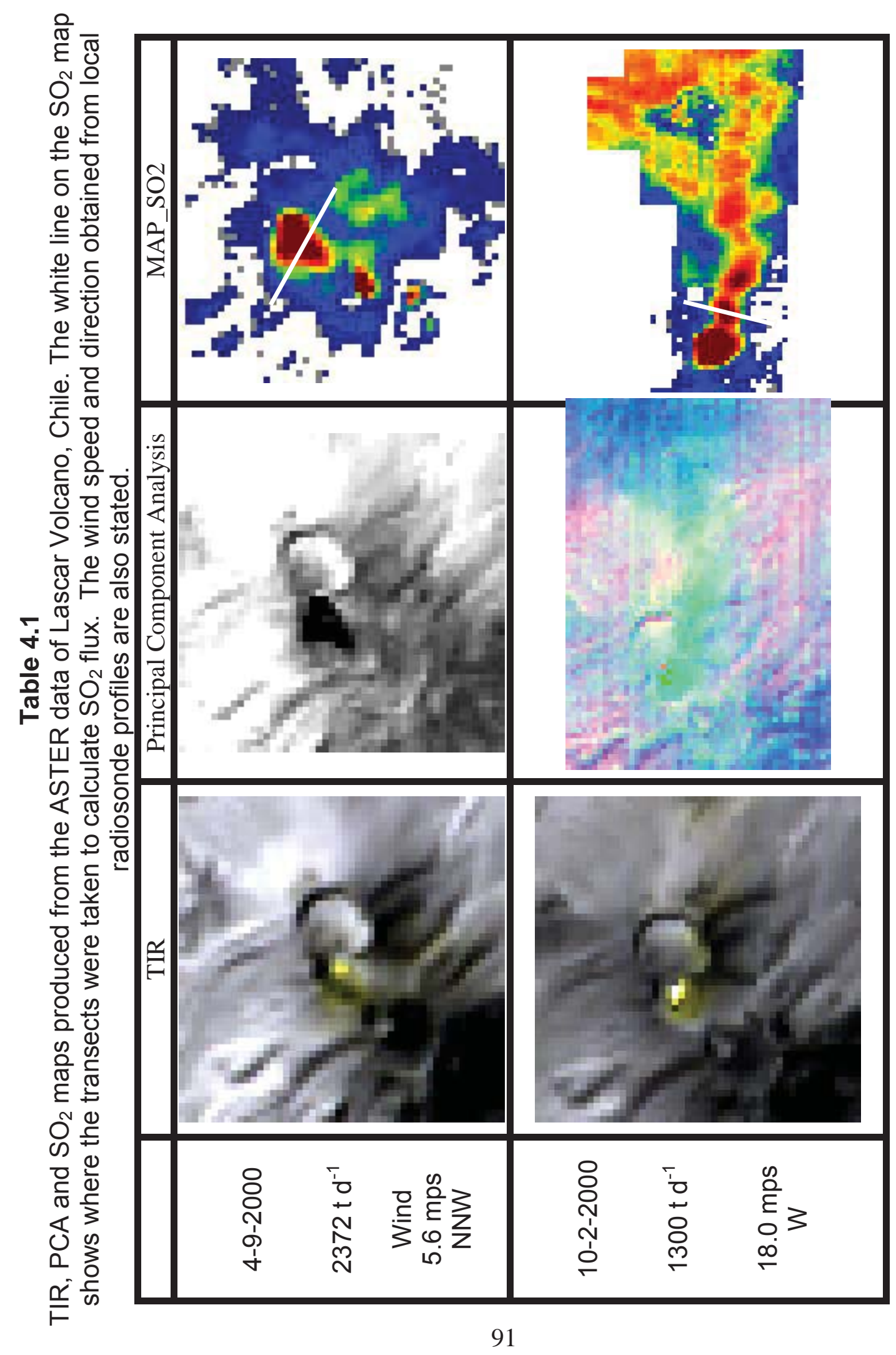




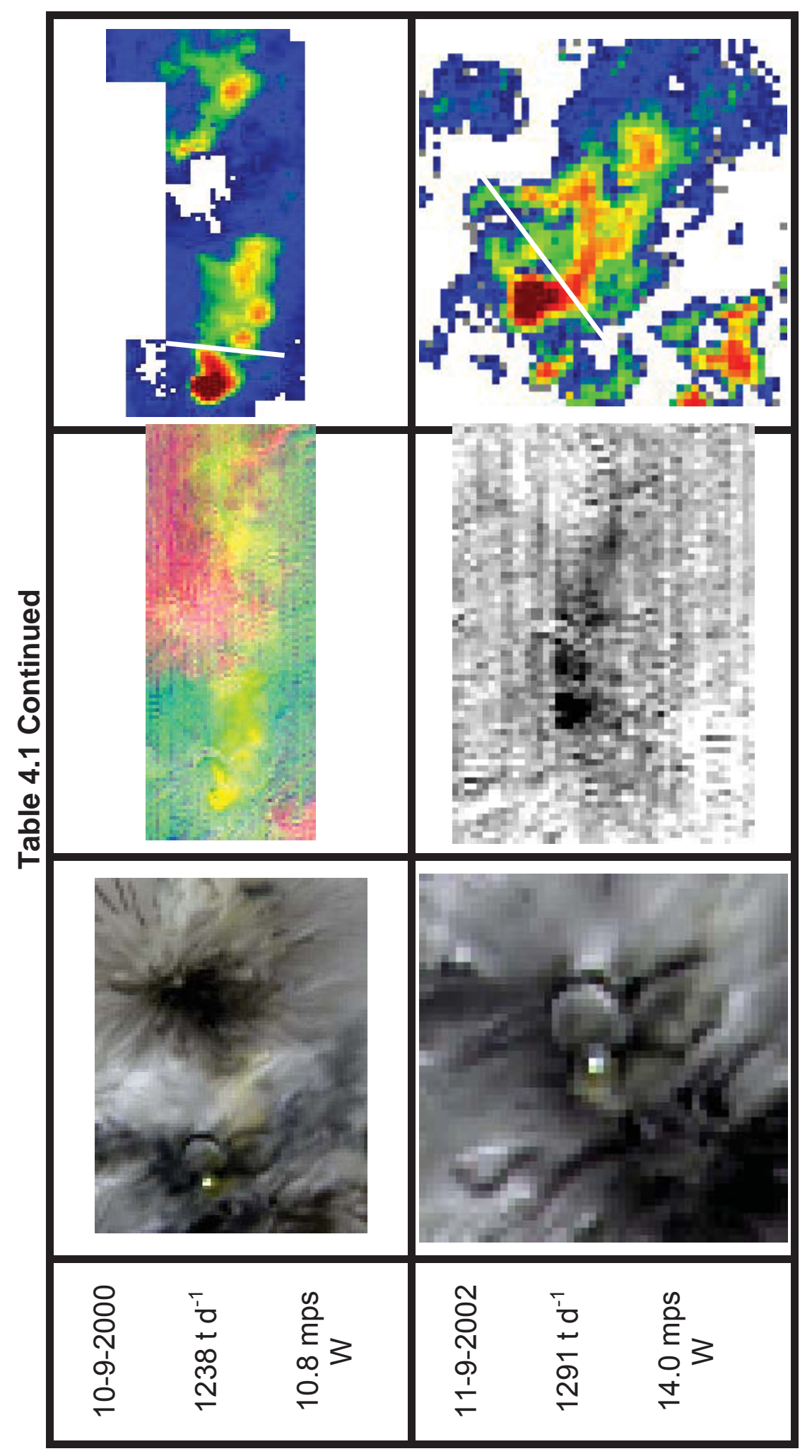




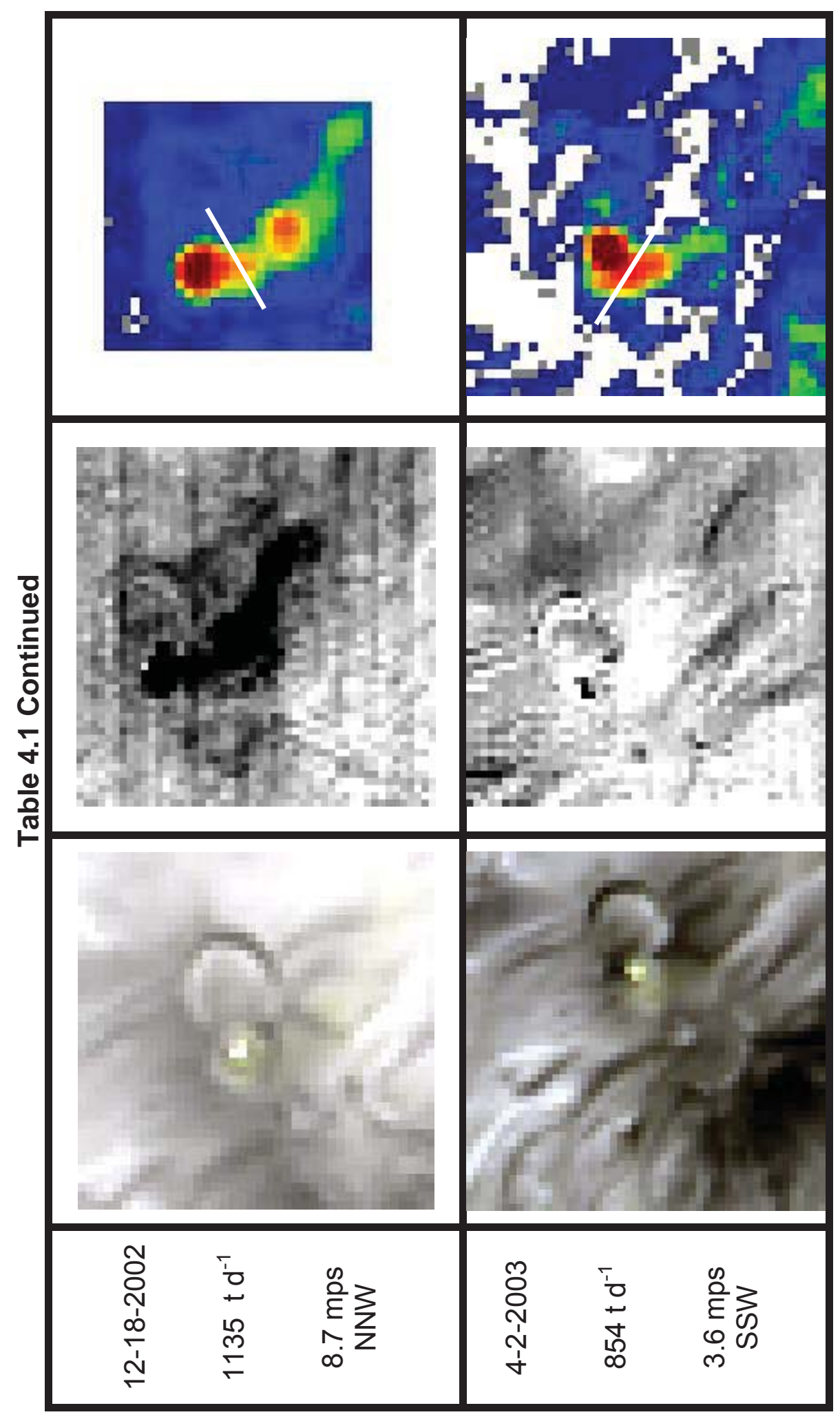




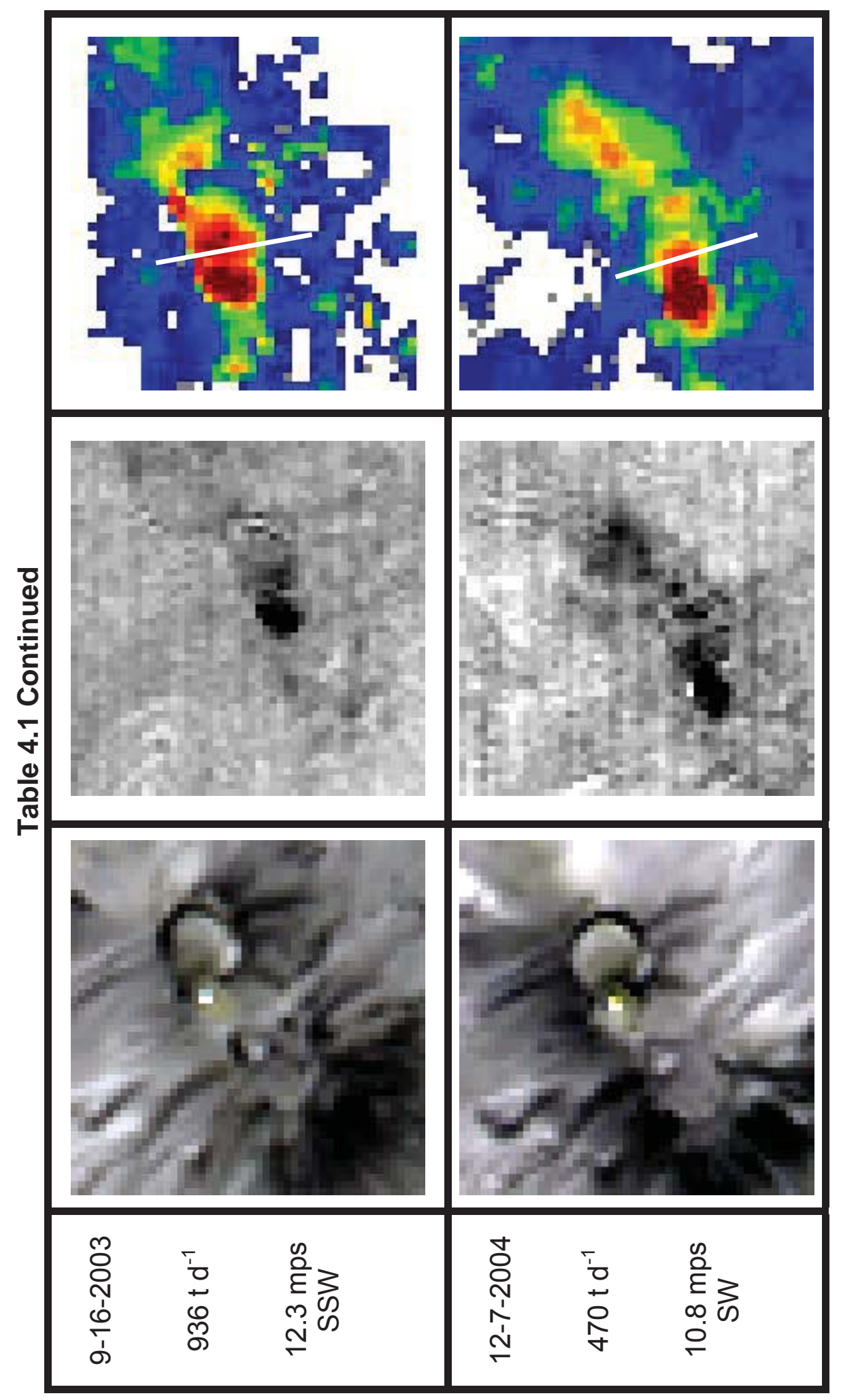




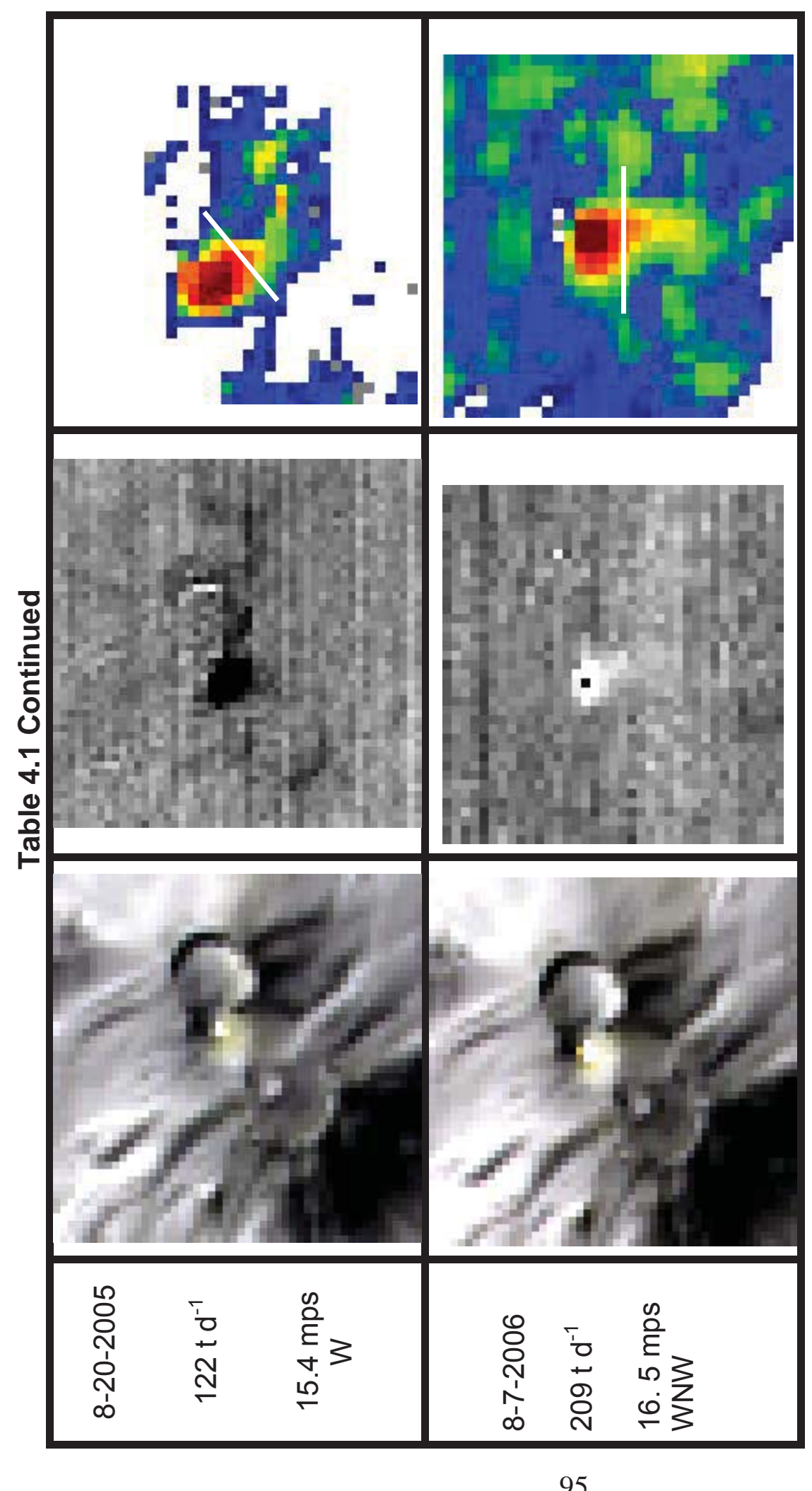




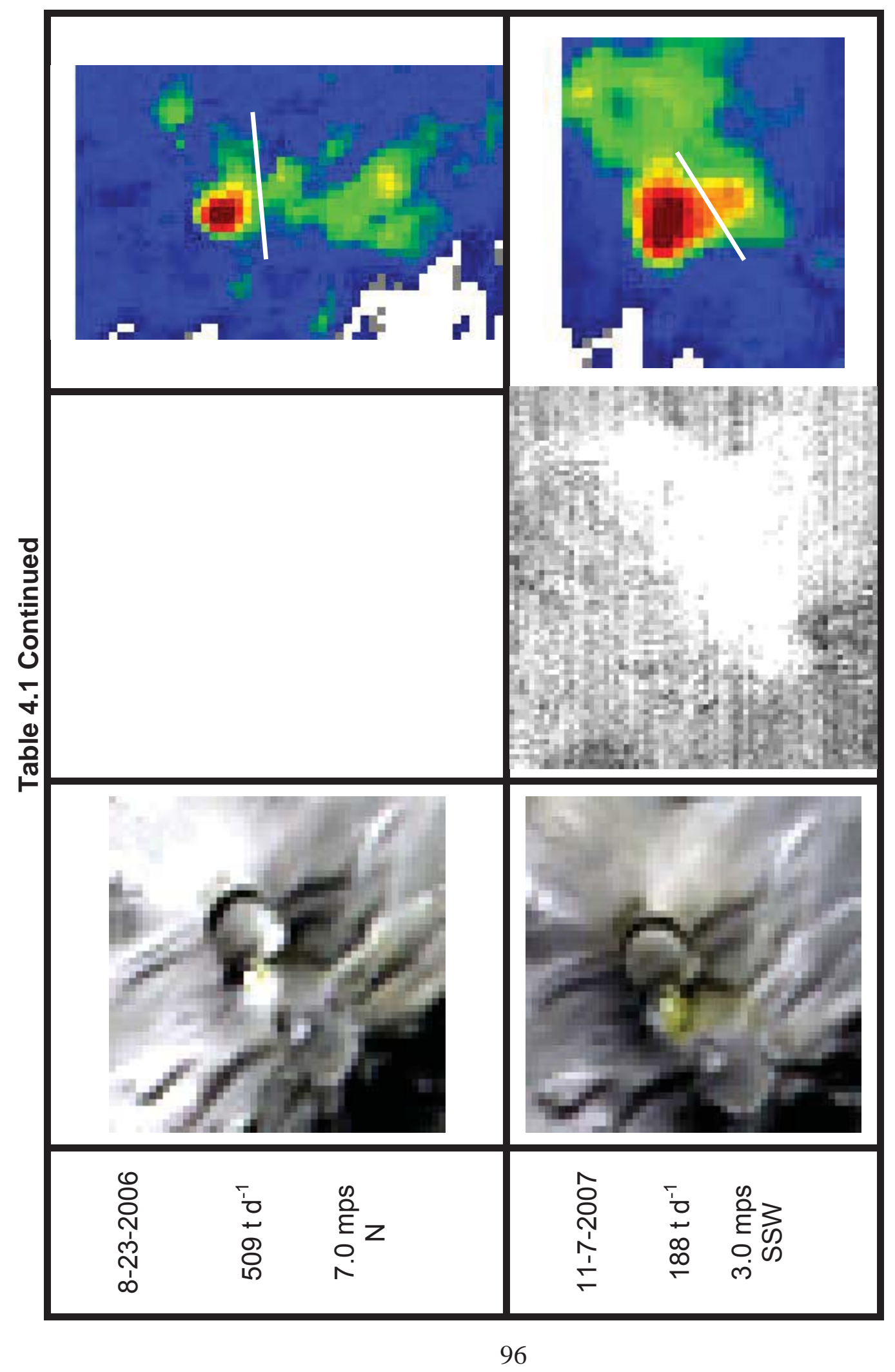




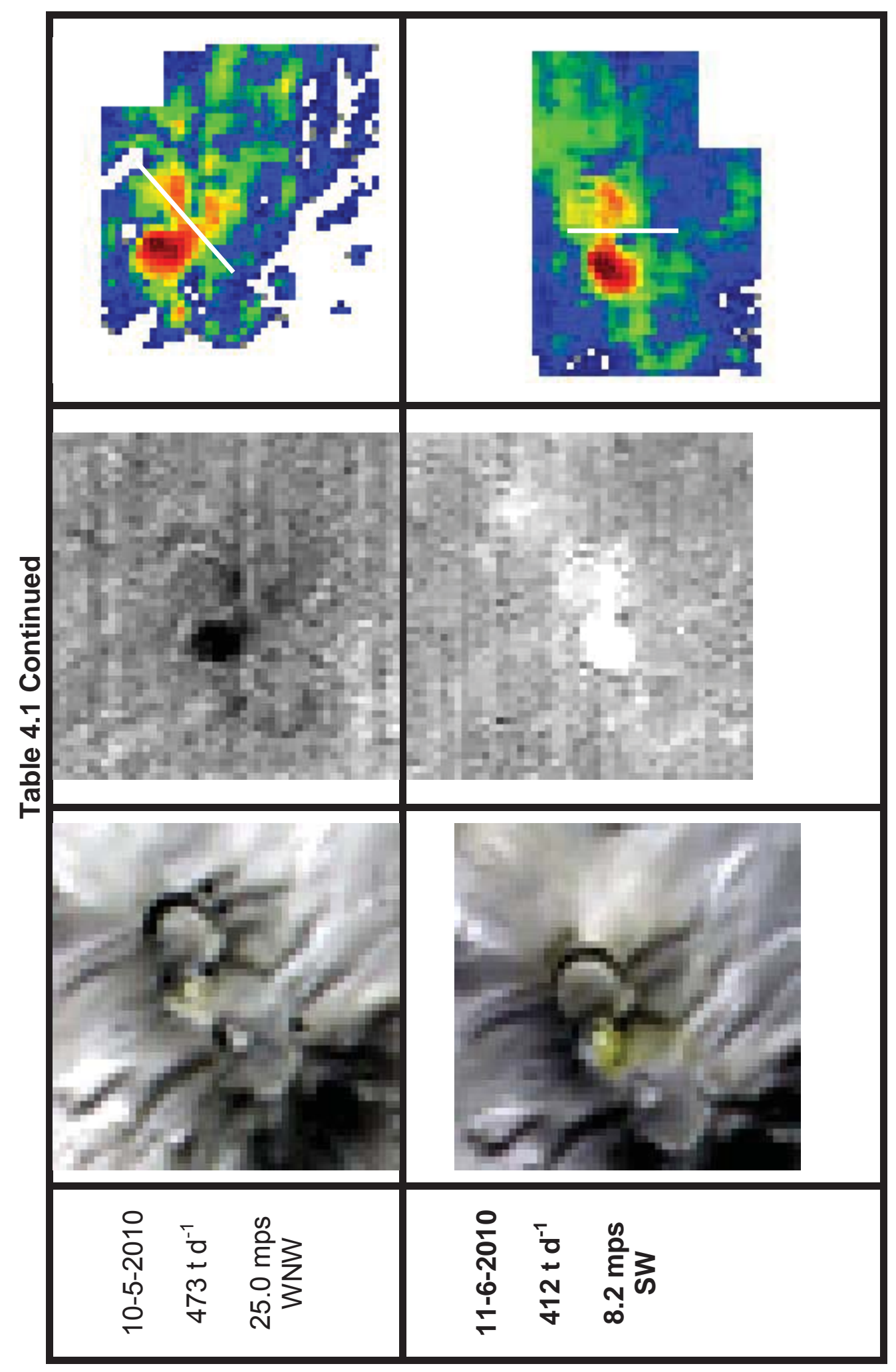




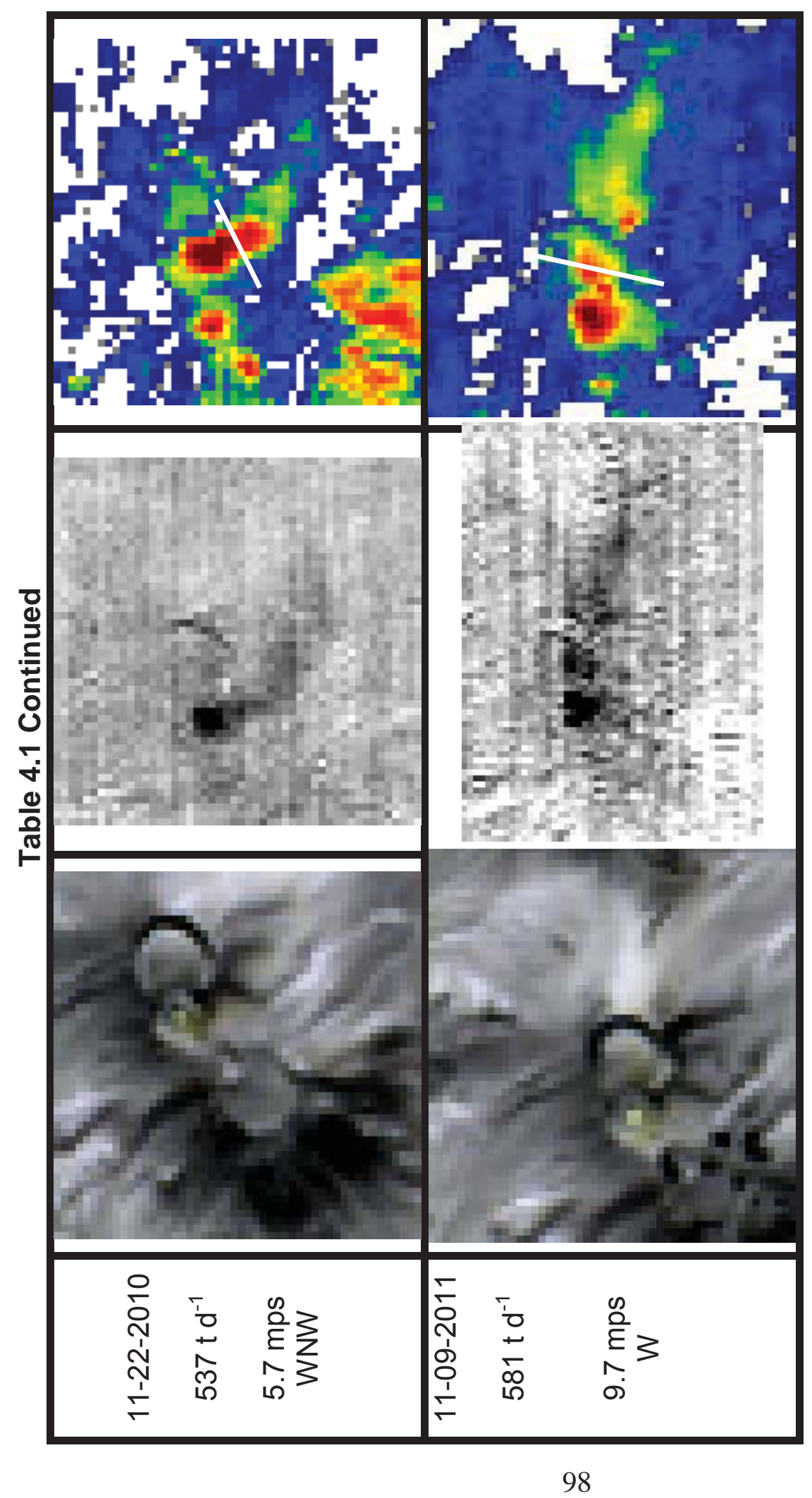




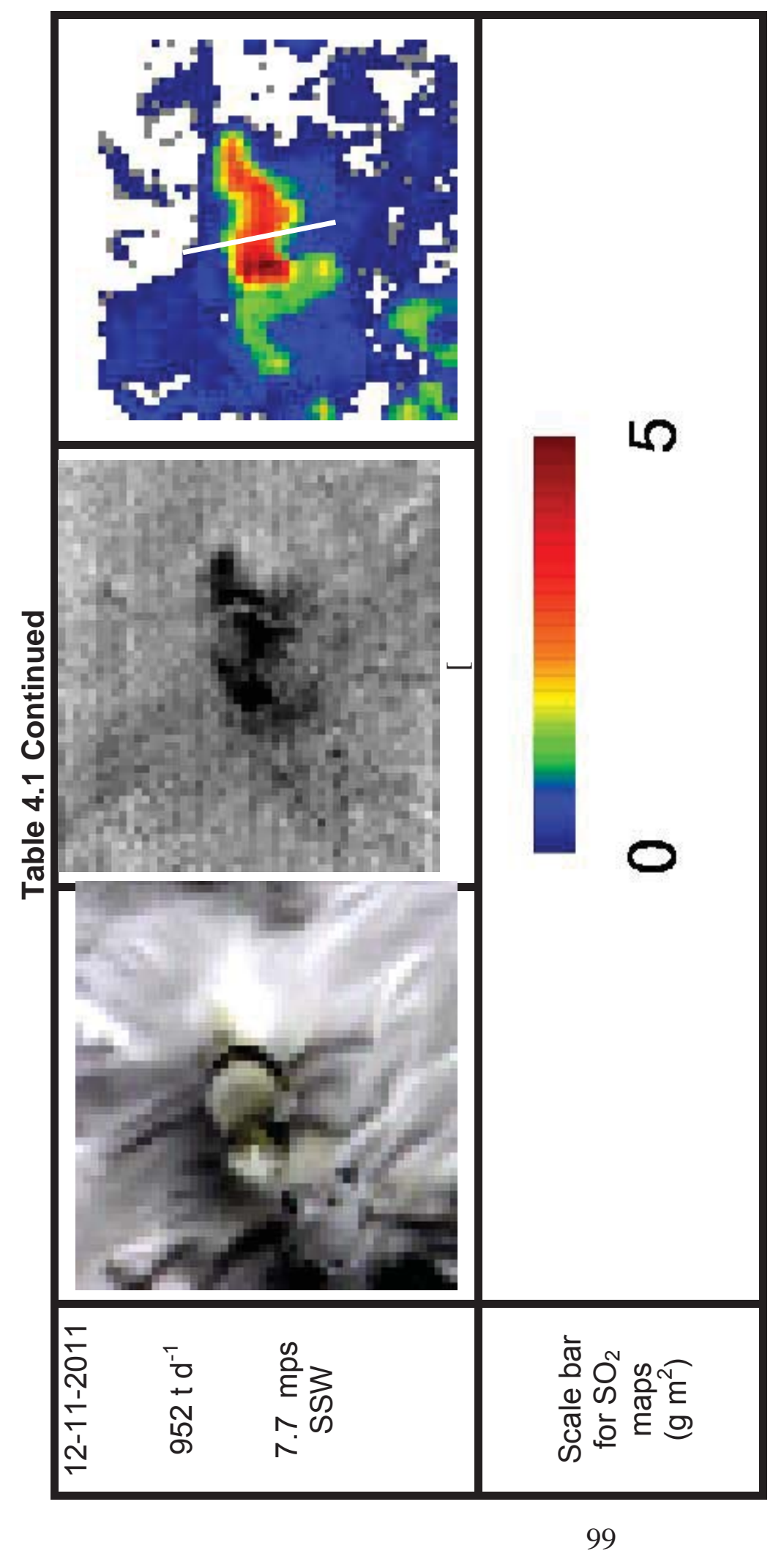


Table 4.2

$\mathrm{SO}_{2}$ maps of Lascar where only $\mathrm{SO}_{2}$ gathered in the crater and no detectable plume was produced.

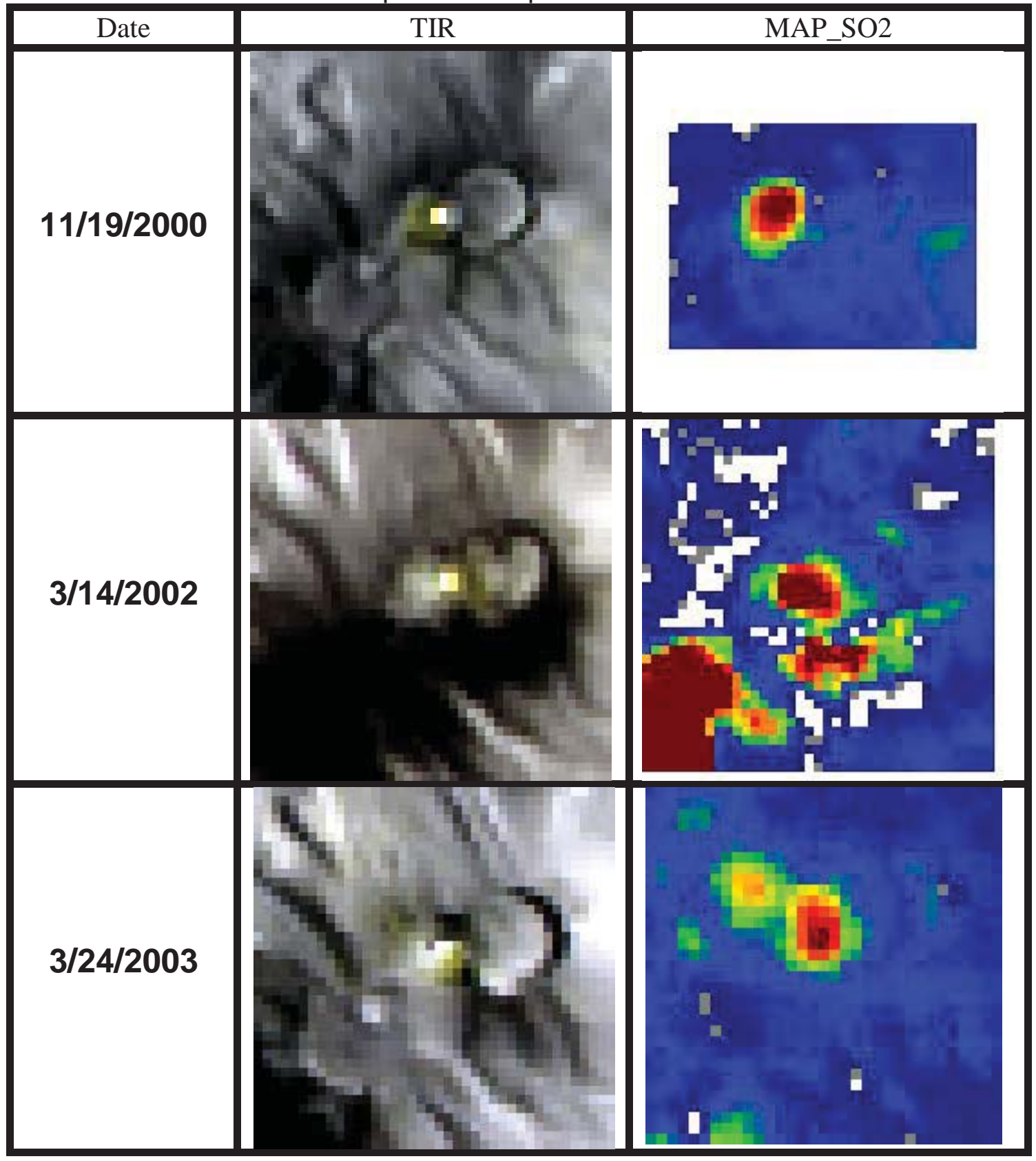


Table 4.2 Continued

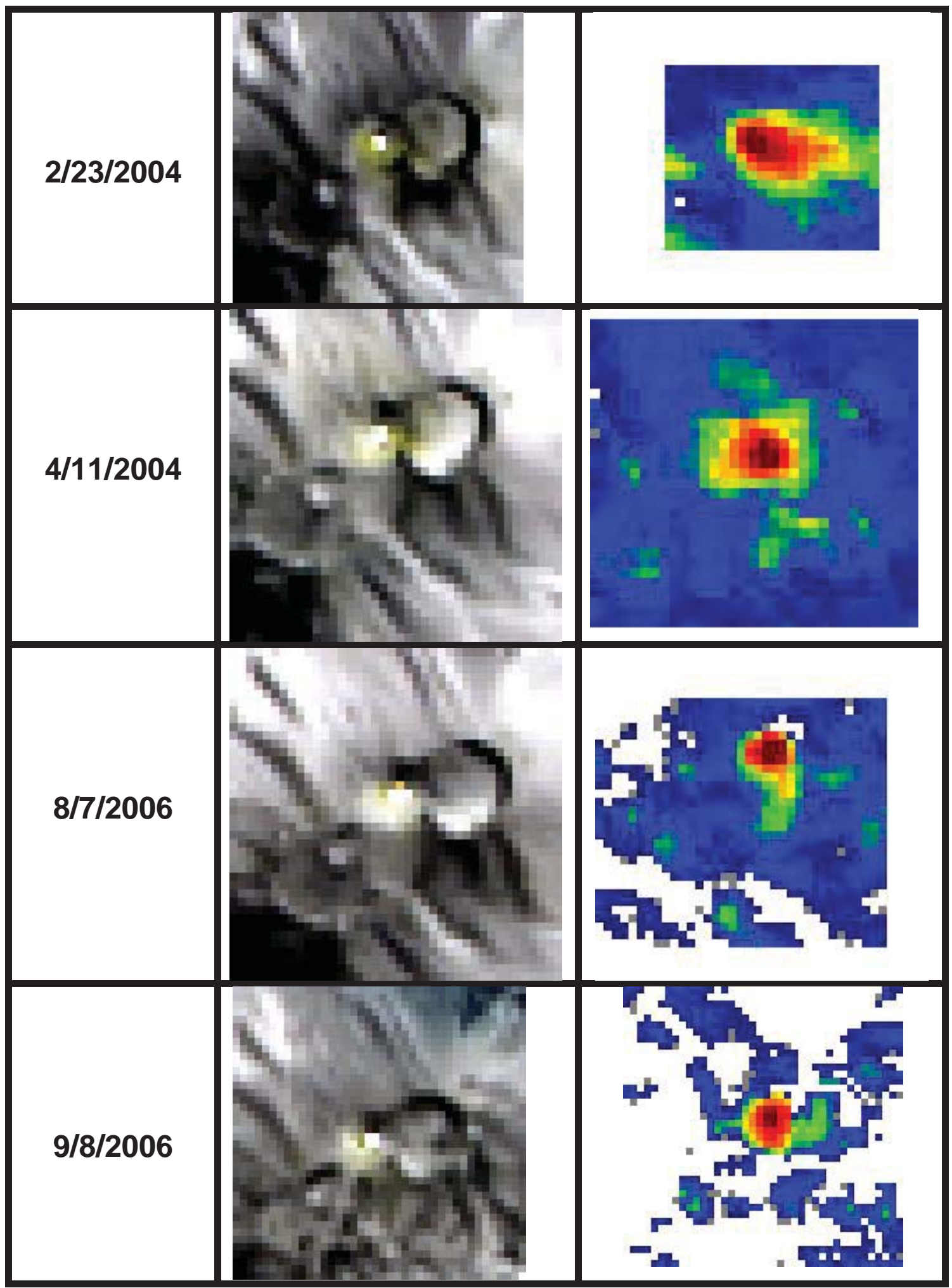


Table 4.2 Continued

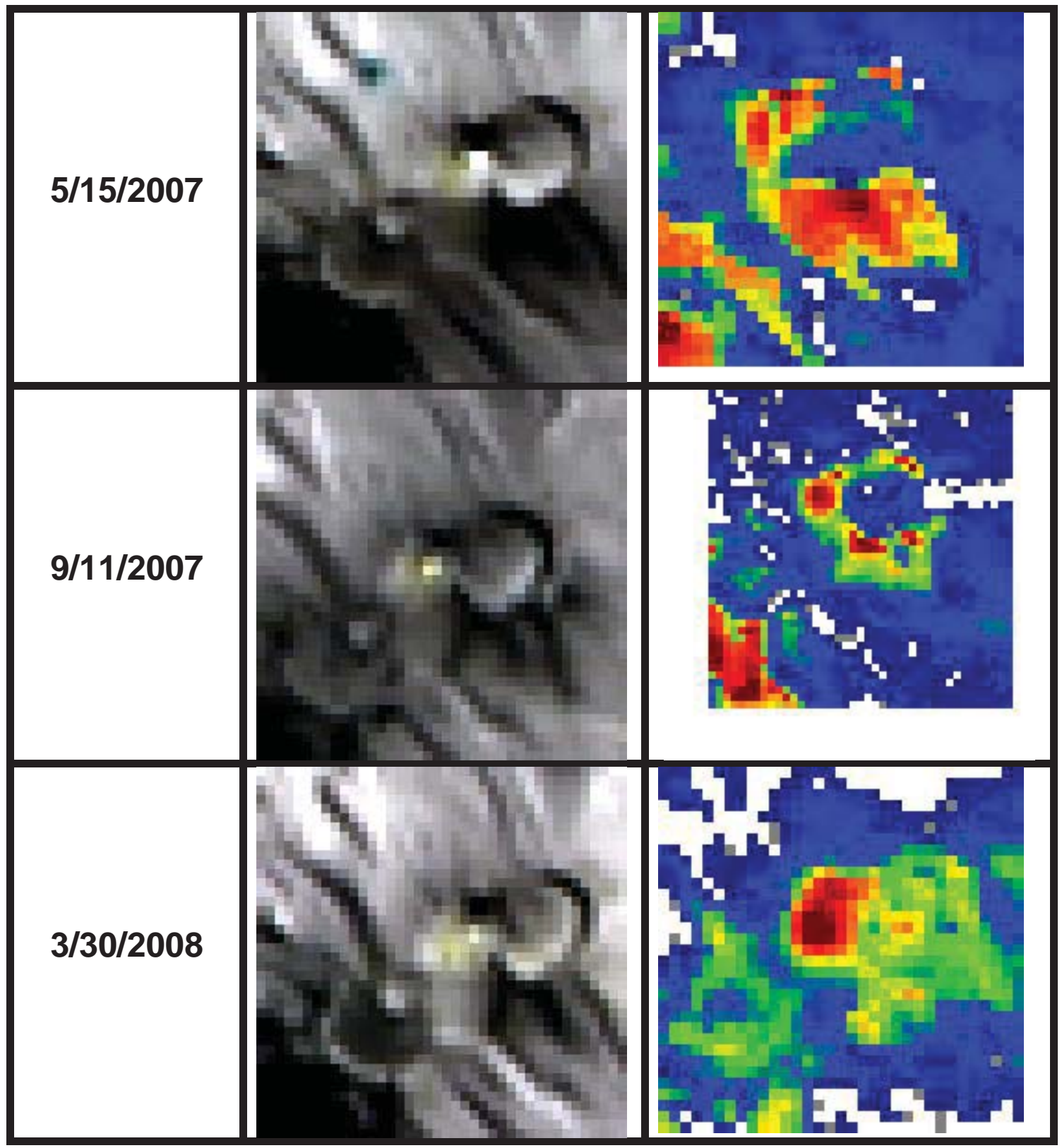


Table 4.2 Continued

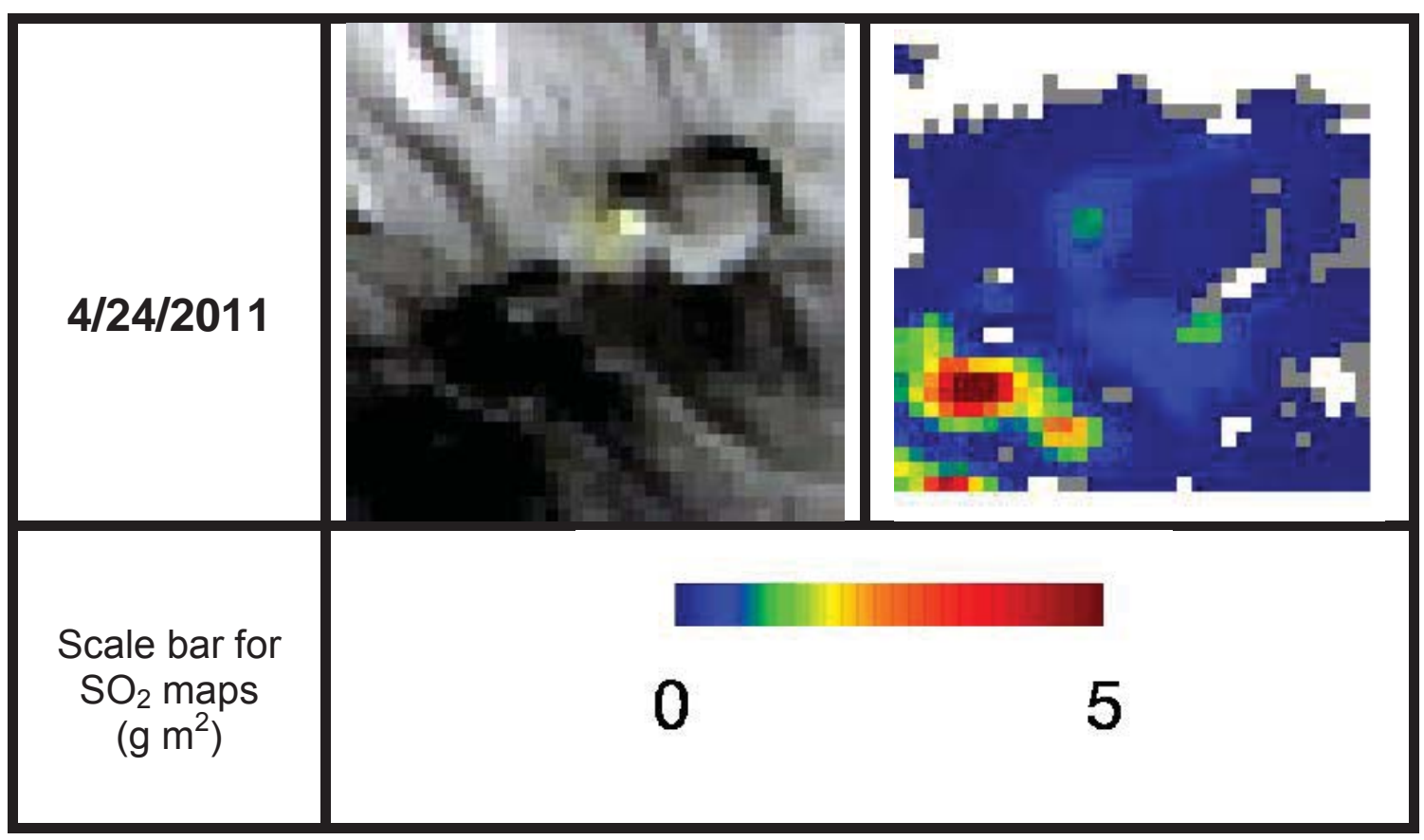

Figure 4.3 illustrates a timeline of activity from Lascar with our $\mathrm{SO}_{2}$ and crater temperature results plotted along with eruptions that have occurred. The timeline also shows ASTER images where $\mathrm{SO}_{2}$ has been identified but cannot be quantified by the MAP_SO2 code. The $\mathrm{SO}_{2}$ fluxes range from $122 \mathrm{t} \mathrm{d}^{-1} \pm 38 \mathrm{t}$ $\mathrm{d}^{-1}$ to $2373 \mathrm{t} \mathrm{d}^{-1} \pm 735 \mathrm{t} \mathrm{d}^{-1}$ with an average of $818 \mathrm{t} \mathrm{d}^{-1} \pm 253 \mathrm{t} \mathrm{d}^{-1}$. It should be noted that the $\mathrm{SO}_{2}$ measurement from 23 January $2003\left(2300 \mathrm{t} \mathrm{d}^{-1}\right)$ was obtained by Mather et al. (2004) using DOAS and is not derived from ASTER. A DOAS measurement was obtained on 7 December 2004 at the same time as an ASTER overpass (Chapter 2). For the purpose of this study the ASTER $\mathrm{SO}_{2}$ flux is used. Daily OMI images were analyzed from 2004 onwards to fill in the gaps where no ASTER images were available or did not detect any $\mathrm{SO}_{2}$. 
Plumes were detected in 6 OMI images from May 2005 and 2009-2011. The images were processed using the OMI PLOT algorithm (refer to Chapter 1 for details). The OMI-derived $\mathrm{SO}_{2}$ fluxes ranged from 277 to $830 \mathrm{t} \mathrm{d}^{-1}$ and were calculated by taking a transect through the plume close to the vent. An example of a plume seen at Lascar by OMI is illustrated in Figure 4.4. Eruptions are included on Figure 4.3 and are plotted according to eruption column height. Table 4.3 provides the dates of the eruptions and their height above the crater.

From Figure 4.3 general trends can be seen. There has been a gradual decline in emissions of $\mathrm{SO}_{2}$ from Lascar with emissions well in excess of $1000 \mathrm{t} \mathrm{d}^{-1}$ from early 2000 to 2003. Emissions from 2003 slowly decreased to $122 \mathrm{t} \mathrm{d}^{-1}$ in August 2005. From 2008 onwards there has been an increase in $\mathrm{SO}_{2}$ emissions with fluxes fluctuating between 400 and $950 \mathrm{t} \mathrm{d}^{-1}$ until the end of 2011. 


\section{Table 4.3}

Dates of the eruptions at Lascar and eruption column heights above the vent. Sources of data: BGVN (2000-2007); Mather et al. (2004)

\begin{tabular}{|c|l|}
\hline Eruption Date & \multicolumn{1}{|c|}{ Height above crater } \\
\hline $7 / 20 / 2000$ & $4-5 \mathrm{~km}$ \\
\hline $10 / 26 / 2002$ & $300 \mathrm{~m}$ \\
\hline $10 / 27 / 2002$ & $1.5 \mathrm{~km}$ \\
\hline $1 / 17 / 2003$ & Unknown, but eruption was reported as minor. \\
\hline $12 / 10 / 2003$ & $400 \mathrm{~m}$ \\
\hline $5 / 4 / 2005$ & $10 \mathrm{~km}$ \\
\hline $4 / 18 / 2006$ & $10-15 \mathrm{~km}$ \\
\hline $4 / 20 / 2006$ & $2.5 \mathrm{~km}, 7 \mathrm{~km}$ \\
\hline $4 / 21 / 2006$ & $3 \mathrm{~km}$ \\
\hline $8 / 14 / 2006$ & Eruption reported as a minor eruption. \\
\hline $9 / 21 / 2006$ & $200 \mathrm{~m}$ \\
\hline $10 / 20 / 2006$ & $200-800 \mathrm{~m}$ \\
\hline $3 / 11 / 2007$ & $5.5-6.5 \mathrm{~km}$ \\
\hline $1 / 1 / 2007$ & above $10 \mathrm{~km}$ \\
\hline $5 / 23 / 2007$ & $9 \mathrm{~km}$ \\
\hline $7 / 18 / 2007$ & $7.6-9 \mathrm{~km}$ \\
\hline
\end{tabular}




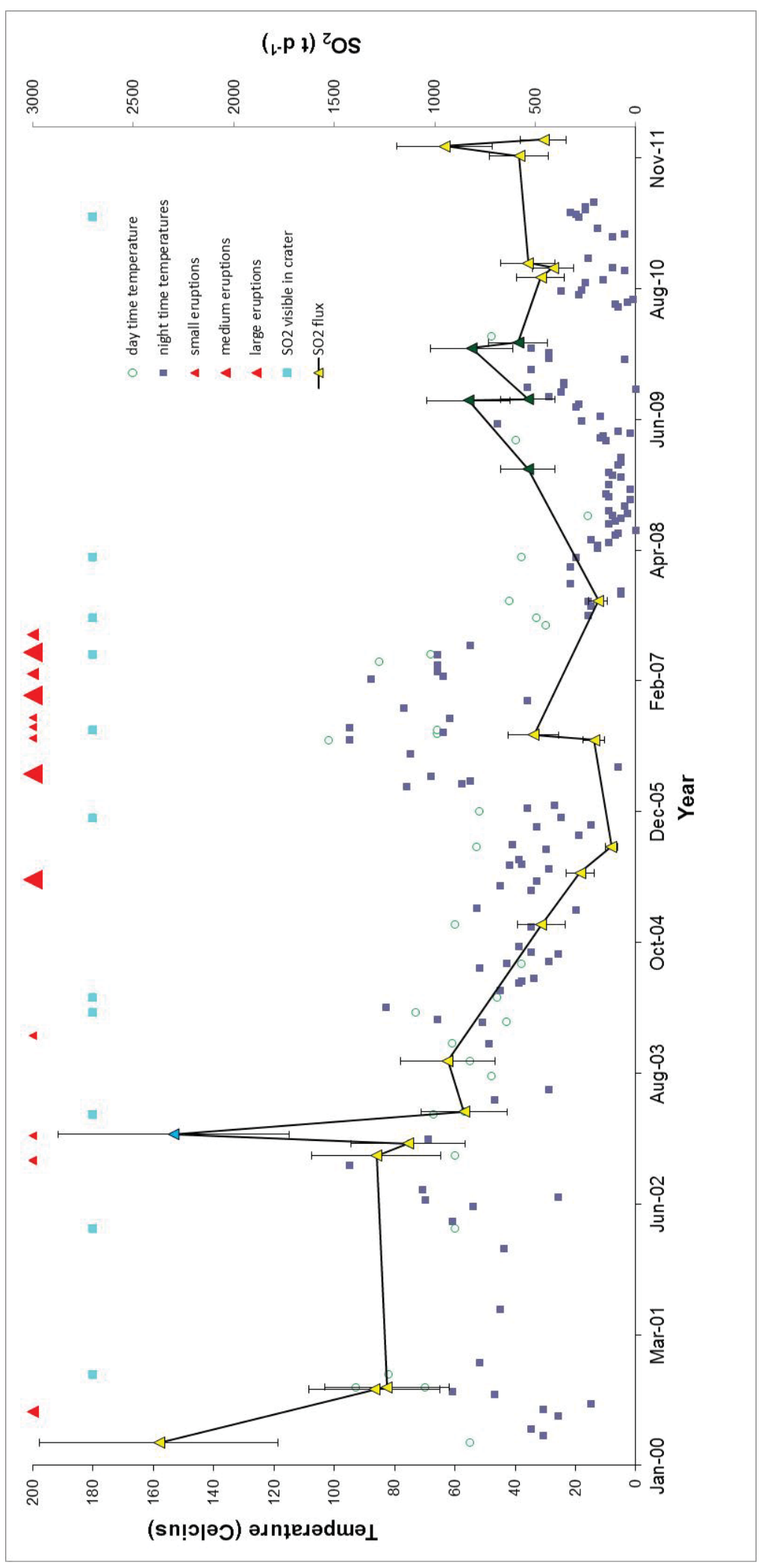

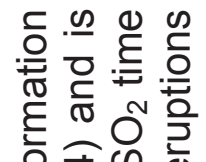

응

드유워 임

으듬

응 㲾

는 $\frac{0}{0} \frac{0}{\frac{C}{\sigma}}$

का ब ह

듣다

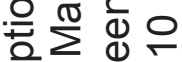

를현

बำ 인 잉

은 는

宁. 또

(ब) $\mathrm{m}$

퓽ㅇㅇㅇ 웡으

O 즁

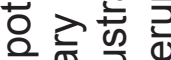

क 즐

으 즘

ल $\frac{\pi}{2} \cdot \frac{\pi}{0}$

क

으 $N$ 응

क

हE

(1) 104

क 華

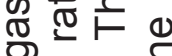

두 i

이음 원

言为

근

苋

ల్

तै

\lrcorner $\mathcal{F}$

후잉

소워

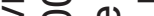

엉 응

ธิ।

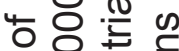

(1) 워 응

느으응

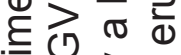

लं 0 ठ ठ

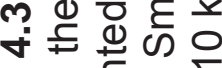

๑ ع ठ

흑 든 을

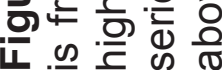




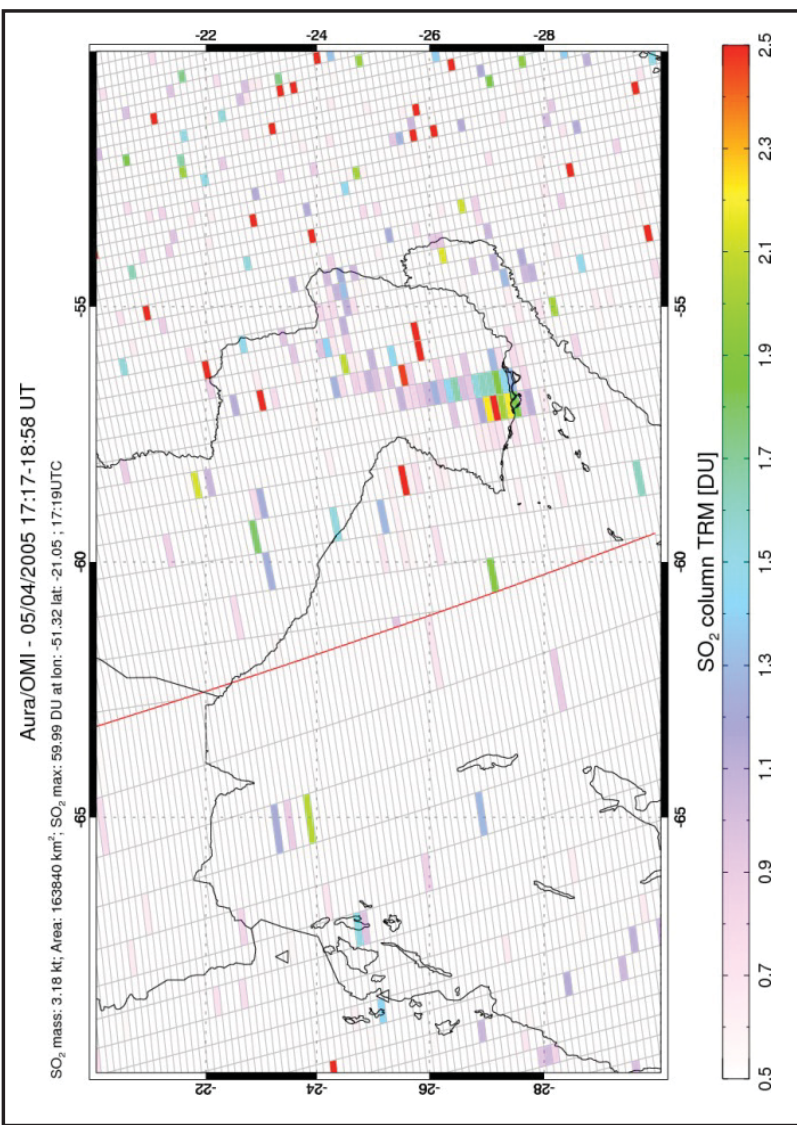

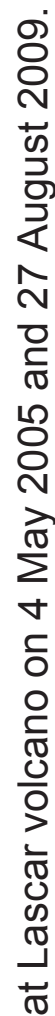

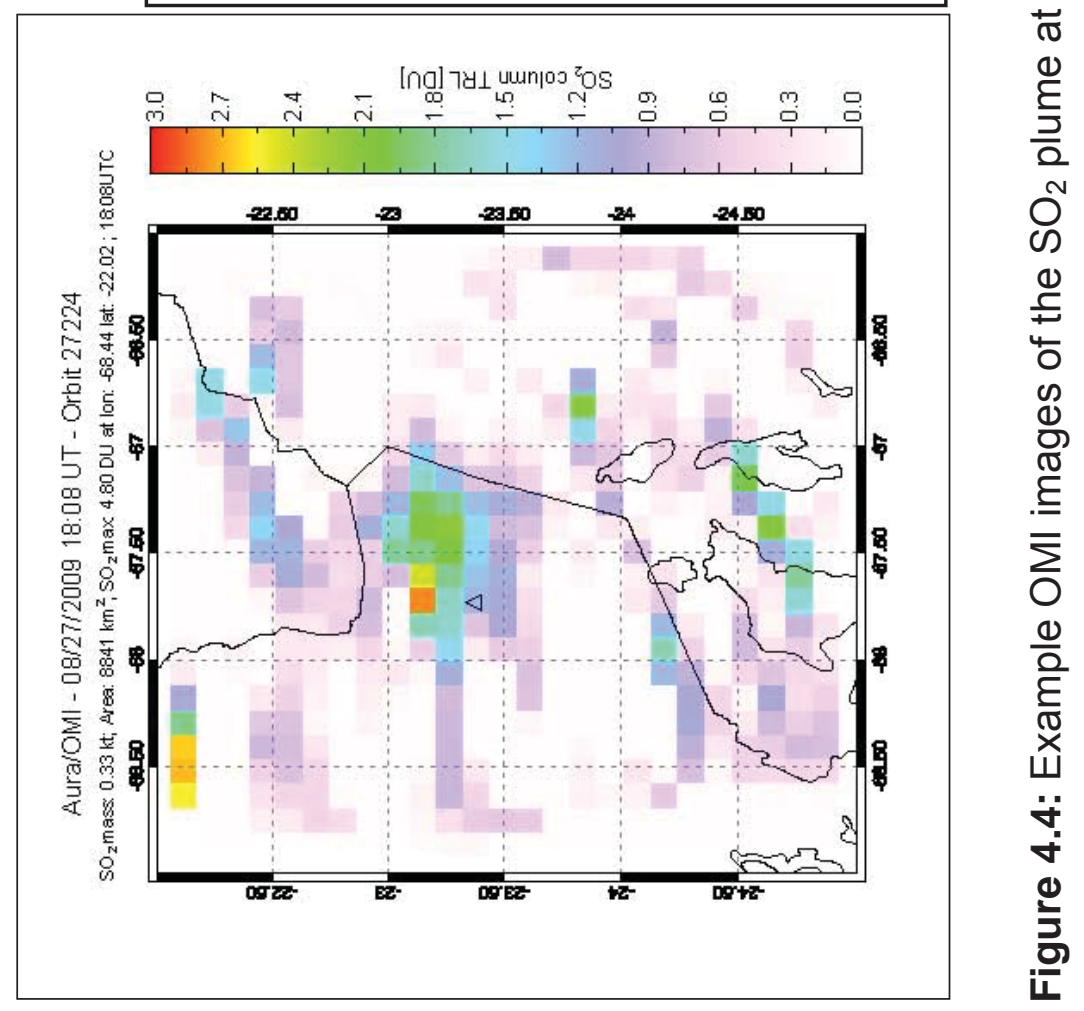




\subsubsection{Maximum crater temperature at Lascar}

The temperature of the thermal anomaly in Lascar's active crater was determined using ASTER's Surface Kinetic Temperature product. Our results were in degrees Celsius, and the daytime and nighttime temperatures were plotted separately on Figure 4.3 due to diurnal factors affecting the data (Murphy et al. 2011). This is easily observed in the data with daytime temperatures being higher than nighttime temperatures.

The temperatures range from $0^{\circ} \mathrm{C}$ to $102^{\circ} \mathrm{C}$. Like the $\mathrm{SO}_{2}$ emissions, the temperatures generally decreased over time from mid-2007 until the end of our time series in 2011. Figure 4.3 does indicate that there are distinct trends in the data and not just a random spread. The most apparent trend is the increase of temperature over the course of a year from late 2005 until 7 August 2006, when a maximum temperature of $102^{\circ} \mathrm{C}$ is recorded prior to a series of eruptions. This increase in temperature could represent an increase in pixel coverage by hot material, elevating the pixel temperature. After these eruptions occurred, the temperature declined. The temperature also increases before the eruption recorded on the 26 October 2002. Two weeks prior to this eruption, a temperature of $95{ }^{\circ} \mathrm{C}$ was recorded. The temperature of the crater declined after this event.

It is not clear what the hotspots represent in the ASTER images. The hotspots potentially represent a volcanic dome or hot fumaroles the crater floor. The size of the thermal feature creating the hotspot is poorly constrained. Is the thermal feature in question occupying the whole pixel or is it much smaller? If the volcanic feature is smaller than a pixel, then the pixel in question is thermally mixed. Hence, temperatures recorded for the hot pixel reflect a combination of the hot volcanic feature and the cooler background temperature. The temperature of the pixel is dependent on how hot and large the volcanic feature 
is. The hot material also might be obscured by volcanic gas or clouds. Thermally mixed pixels are discussed further below. 


\subsection{Discussion}

\subsubsection{Changes in $\mathrm{SO}_{2}$ emissions and Hotspot Temperatures (Figure 4.3)}

It is unknown if Lascar has started a new cycle, with dome growth and subsidence, at some point over the past 11 years. Visual observations have been infrequent throughout this time. No dome was observed at Lascar in November 2002 (BGVN 2002), May 2005 (BVGN 2005), or December 2009 (Murphy et al. 2011). Without continuous observations we cannot be certain that no dome has been emplaced at some point over the past 11 years. Additional information such as $\mathrm{SO}_{2}$ flux must be relied upon along with changes in the thermal radiance.

The most obvious trend in the $\mathrm{SO}_{2}$ time series (Figure 4.3) is a decline in flux, with a maximum of $2373 \mathrm{t} \mathrm{d}^{-1}$ emitted on 9 April 2000, and a minimum of $122 \mathrm{t}$ $\mathrm{d}^{-1}$ emitted on 20 August 2005. After the 2006/2007 eruption sequence, the emissions began to rise and become more stable, fluctuating between $450-$ $950 \mathrm{t} \mathrm{d}^{-1}$. The hotspot temperatures generally decreased with time with the exception of the 2006-2007 eruption series (Figure 4.3). Notable trends in the hotspot data include an increase in temperature prior to the 26 October 2002 eruption and the 2006/2007 eruption series. A decrease in temperature is seen during and after the 2006/2007 eruption series. An increase in temperature is seen after the minor eruption in December 2003. Another study by Murphy et al. (2011) carried out similar work using the ASTER kinetic temperature data product to analyze changes in the crater temperature at Lascar. The results found in this study are mostly in agreement with Murphy et al. (2011), illustrating similar trends. The major difference between our data set and that produced by Murphy et al. (2011) is the number of data points. In this study all the data points are considered however in the study by Murphy et al. (2011) the hot pixel in question must be significantly warmer than the background temperature to be considered. Figure 4.5 illustrates the results from Murphy et al. (2011) in comparison to ours. 


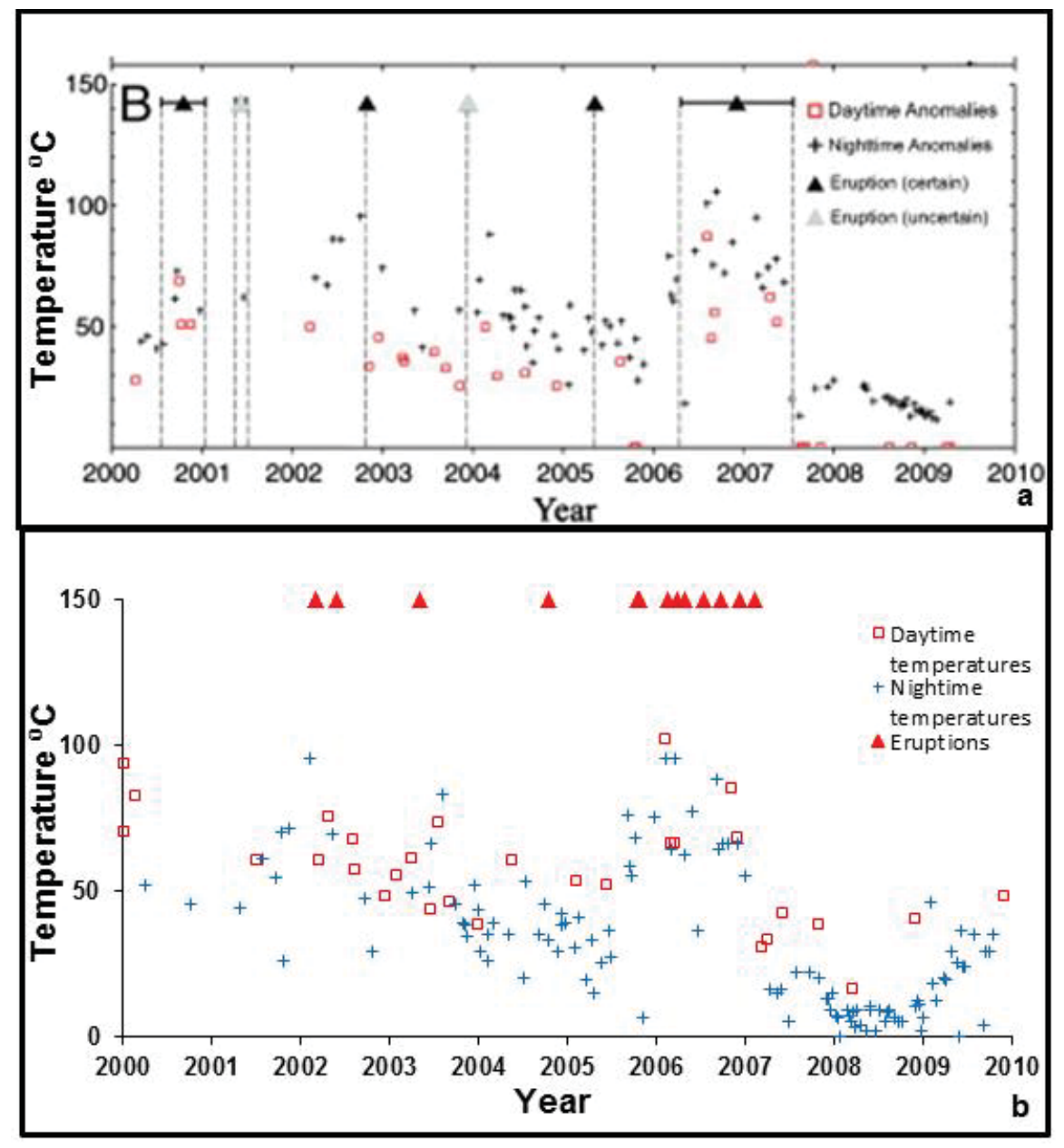

Figure 4.5: The ASTER temperatures produced by Murphy et al. (2011) (a) in comparison to our temperatures produced from the ASTER data (b). 
Based on the $\mathrm{SO}_{2}$ data points between April 2000 and January 2003 it is assumed that emissions were high $\left(>1000 \mathrm{t} \mathrm{d}^{-1}\right)$ during this period of time. Throughout this time 3 relatively small-medium sized eruptions occurred. Prior to the 20 July 2000 eruption, a SO 2 flux of $2373 \mathrm{t} \mathrm{d}^{-1}$ was quantified from ASTER on 9 April 2000. It is clear that the conduit during April 2000 was not obstructed, making it easy for gas to escape from the magma. After this eruption the $\mathrm{SO}_{2}$ flux dropped to $\sim 1300 \mathrm{t} \mathrm{d}^{-1}$ in early October 2000 (ASTER measurements acquired on 1 and 9 October 2000). Due to the lack of data prior to this eruption trends in the temperature, and $\mathrm{SO}_{2}$ data to a certain extent, are not obvious. However, a high crater temperature of $93^{\circ} \mathrm{C}$ recorded on 9 October 2000 can be attributed to an increase on $\mathrm{SO}_{2}$ from the high temperature fumaroles (Murphy et al. 2011). Visual observations made in November 2002 indicated that after the July 2000 eruption, the remnants of the 1993 dome had subsided even further in to the crater. There was no evidence of a new dome being emplaced after the July 2000 eruption (BGVN 2003). The BGVN report (2003) suggested that Lascar, during this period of time, was at or near the climax of its dome subsidence phase.

Andres et al. (1991) stated that Lascar emitted an average of $2300 \mathrm{t} \mathrm{d}^{-1} \mathrm{SO}_{2}$, and the ASTER measurement from April 2000 is consistent with this estimate. Measurements by Andres et al. (1991) ranged from 540-6970 $\mathrm{t} \mathrm{d}^{-1}$ over a 2 day period in November 1989, with over 90 COSPEC measurements having been taken. This suggests that high emission rates can occur during periods of passive degassing and fluxes can vary greatly over short periods of time. This suggests that the fluxes obtained by ASTER and OMI may vary greatly before and after the time when the data was acquired. TOMS data suggest that 400000 tons of $\mathrm{SO}_{2}$ was emitted during the December 1993 eruptive event. In comparison, the passive degassing emissions quantified for April 2000 and November 1989 are relatively small. 
Temperatures increased prior to the 26 October 2002 eruption, with a maximum temperature of $95^{\circ} \mathrm{C}$ recorded on 5 October 2002. Murphy et al. (2011) suggest that this increase in thermal output represents an increase in the flux and/or temperature of the gases emitted from the fumaroles at Lascar. No $\mathrm{SO}_{2}$ flux data is available prior to the eruption when the thermal flux is high. $\mathrm{SO}_{2}$ fluxes after the eruption were $1300 \mathrm{t} \mathrm{d}^{-1}$ (9 November 2002 ASTER measurement). It was reported that the crater collapsed following this eruption (BGVN 2002).

A decrease followed by a significant increase in $\mathrm{SO}_{2}$ flux is evident with a minor eruption that occurred on 17 January 2003 (Mather et al. 2004). An ASTER measurement acquired roughly 1 month before the eruption had a flux of $1135 \mathrm{t}$ $\mathrm{d}^{-1}$. A DOAS measurement the day after the eruption showed an increase in $\mathrm{SO}_{2}$, with a flux of $2300 \mathrm{t} \mathrm{d}^{-1}$ (Mather et al. 2004) (Figure 4.3). It is speculated the eruption cleared the conduit temporarily allowing gas to escape more easily. After this eruption, gas emissions steadily dropped reaching a low of $122 \mathrm{t} \mathrm{d}^{-1}$ on 20 August 2005.

A small eruption occurred on 10 December 2003. After this eruption the temperature of the crater gradually increased until March 2004 (Figure 4.3). Murphy et al. (2011) suggested that the increase in thermal flux was either a direct result of a post-eruption increase in fumarolic activity or the presence of an active lava dome. They suggest that the decompression of magma in the upper conduit, following the expulsion of magma from the December 2003 eruption, assisted the exsolution and transport of volatiles to the surface. This may have been the case after the January 2003 eruption where $\mathrm{SO}_{2}$ emissions briefly increased. Due to ASTER's temporal resolution, data was not available for the days to weeks preceding the December 2003 eruption to determine if emissions did increase. From Figure 4.3 it can be seen that the general trend is a decrease in $\mathrm{SO}_{2}$ emissions. 
The eruption on 4 May 2005 did not exhibit any thermal anomalies prior to the eruption or after. It was revealed from a visit to the crater, on 25 May 2005, that there had been a crater collapse and 3 new fumaroles were evident around the crater (BGVN 2005; Murphy et al. 2011). With the thermal anomalies decreasing after this eruption, it has been suggested that the collapse covered the crater floor, blocking any IR radiance from hot material (Murphy et al. 2011). Other possible mechanisms for a decrease in crater temperature are reduced conduit permeability due to the precipitation of hydrothermal minerals or an increase in the viscosity of the lava due to cooling and welding and fusion to the conduit walls (Matthews et al. 1997; Sparks 1997; Murphy et al. 2011). Gas emissions dropped to $122 \mathrm{t} \mathrm{d}^{-1}$ several months after the eruption (ASTER measurement 20 August 2005) which is consistent with a reduction in permeability within the conduit, making it difficult for gases to escape. It is unknown what changes occurred to the gas emissions before and during the eruption since no ASTER imagery is available. Two OMI images detected $\mathrm{SO}_{2}$ emissions from Lascar, one from the same day of the eruption and the other weeks after on the 26 May 2005 (Figure 4.4). The plume imaged on the 4 May 2005 had drifted and is located over SE Paraguay. The 26 May $2005 \mathrm{OMI}$ image provided a flux of $277 \mathrm{t} \mathrm{d}^{-1}$. This flux is not as high as expected after a large eruption, but does emphasize the declining trend in $\mathrm{SO}_{2}$ (Figure 4.3) and indicates that the permeability of the system is reduced and the conduit is sealing. The 2005 eruption itself may have been a direct result of the pressure in the conduit building up due to the system sealing and trapping volatiles.

One of the most apparent trends seen at Lascar is the increase in temperatures building up to the 2006-2007 eruptive episode, followed by a decrease in temperature. Temperatures rose from $53^{\circ} \mathrm{C}$ on 16 December 2005 to $102^{\circ} \mathrm{C}$ on 8 July 2006. The temperatures then decreased to $16^{\circ} \mathrm{C}$ over the next 2 years. $\mathrm{SO}_{2}$ emissions also mimic this trend increasing from $122 \mathrm{t} \mathrm{d}^{-1}$ in August 2005 to $509 \mathrm{t} \mathrm{d}^{-1}$ August 2006. The $\mathrm{SO}_{2}$ flux then decreased to $188 \mathrm{t} \mathrm{d}^{-1}$ in November 
2007. Such observations could indicate an increase in permeability of the conduit or the emplacement of a lava dome. If a lava dome was emplaced it was likely destroyed by the eruptive episode in 2006. An alternative to dome emplacement is the introduction of new magma in to the system, which may have been exposed in the conduit, resulting in an increase in temperature and gas flux. A large increase in $\mathrm{SO}_{2}$ emissions $\left(>1000 \mathrm{t} \mathrm{d}^{-1}\right)$ would be expected if new magma was introduced into the system and this is not evident (Matthews et al. 1997).

The eruptions during 2006/2007 could be a response to the sealing of the conduit. The increased pressure building up in the conduit could have triggered the eruptions. The eruptions themselves would have temporarily relieved the pressure and increased the permeability of the conduit, allowing gases to escape more easily. After the eruption series the gas flux is consistently above a flux $450 \mathrm{t} \mathrm{d}^{-1}$ (Figure 4.3). The increase in crater temperatures may be a consequence of the eruptions.

A chemical and isotopic analysis of gases emitted from Lascar's high temperature fumaroles during November 2002, May 2005, and October 2006 was conducted by Tassi et al. (2009). The isotopic and chemical signatures indicated a mixed magmatic, hydrothermal and meteoric source. Before the 2005 eruption, the chemical signatures point towards a magmatic source. After this eruption, the signatures shifted to more of a hydrothermal source than a magmatic one. This suggests that after the May 2005 eruption there was no new magma in the shallow areas below the crater (Tassi et al. 2009). It is unknown if this is the case after 2006, but due to the diminishing $\mathrm{SO}_{2}$ emissions (until 2008) and lower crater temperatures we infer that no dome was present and fresh magma was not being fed in to the shallow plumbing system of the volcano. 
From 2008 onwards measurements indicate that $\mathrm{SO}_{2}$ emissions have increased, and fluctuate between $450-930 \mathrm{t} \mathrm{d}^{-1}$ until the end of 2011. No dome was apparent in December 2009 (Murphy et al. 2011). New magma might have potentially entered the system, resulting in increased $\mathrm{SO}_{2}$ emissions. Another possibility is a second boiling of the magma in the chamber with the crystallization of minerals triggering gas release (Sparks 1997; 2003).

No complete cycles appear to be occurring at Lascar. Visual observations suggest that Lascar was in the final stages of dome subsidence after the July 2000 eruption (BGVN 2003; Mather et al. 2004). If this is indeed the end of the cycle that began in 1993, it would indicate that the cycles are getting longer in duration. The previous 3 cycles had approximate durations of 1-2 years. The $\mathrm{SO}_{2}$ time series data, changes in thermal radiance and visual observations suggest that a dome has been present at any point over the past 11 years. With the general trend of decreasing $\mathrm{SO}_{2}$ emissions and thermal radiance, it is suspected that Lascar's conduit is sealing. With the sealing of the conduit, porosity decreases, preventing gases from escaping. The eruptions are a response due to the increase in pressure as gases are unable to escape. The chemical and isotopic analysis by Tassi et al. (2011) agrees with the suggestion that no new magma has entered the conduit at Lascar. The hot crater temperatures are a direct result of the fumaroles, not an exposed patch of magma or the presence of a volcanic dome. 


\subsection{Conclusions and Further Work}

Gas emissions and temperatures at Lascar are in decline. Gas emissions have dropped from over $2000 \mathrm{t} \mathrm{d}^{-1}$ in 2000 to less than $1000 \mathrm{t} \mathrm{d}^{-1}$ in 2011. Combining the gas data with thermal data has provided a clearer picture of the activity at Lascar, especially in comparison to other studies where gas emissions were neglected. It is apparent that Lascar is at the end of the cycle that started in April 1993. The data indicates that magma may have been injected into the system during or prior to 2000 , providing a source for the extensive degassing. The combination of dropping gas emissions and temperatures of the thermal anomaly in the crater suggests that this magma is becoming depleted in gas and cooling as it evolves. Due to our limited data set it cannot be assumed that no dome has been present over the past 11 years, or that no new magma has entered the conduit. From the gas and temperature data it appears that the system at Lascar is sealing. The eruptions are a response to the gasses becoming trapped, increasing the pressure. The explosions temporally relieve the pressure allowing gasses to escape. If a new batch of magma were to enter the chamber, a new cycle may begin but there is no sign of this occurring from the data presented here.

With ASTER's temporal resolution, and problems with the temperature contrast between the plume and the ground, many plumes might not have been viewed at Lascar. There are several months between measurements at Lascar. OMI did provide images of $6 \mathrm{SO}_{2}$ plumes at Lascar adding to the dataset. $\mathrm{OMI}$ is capable of computing daily $\mathrm{SO}_{2}$ tonnages or time-averages over longer periods. This would be beneficial at Lascar to determine if any $\mathrm{SO}_{2}$ is being emitted during periods of time where ASTER provided no measurements or a single daily image did not detect anything.

It may be beneficial to this work to investigate the maximum single pixel temperatures further. We are uncertain whether the pixels are thermally mixed, 
representing a volcanic feature smaller than the $90 \mathrm{~m}$ pixel size. Work is needed to determine what portion of the pixel is volcanic and what portion is background material. Also the temperature of the volcanic feature needs to be deduced. This can be done by means of the dual band technique used by Dozier (1981), Francis and Rothery (1987), Crisp and Baloga (1990), Oppenheimer et al. (1991), and Wright and Flynn (2003). The at-sensor radiance for a pixel is a weighted average of all the radiators present within the pixel, and is dependent on temperature and wavelength (Wright and Flynn, 2003). The dual band technique is based on the postulation that surfaces such as a lava flow or dome can be described by two end members (radiators): hot cracks radiating at one temperature and homogenous crust radiating at a lower temperature (Rothery 1988; Francis and Rothery 1987; Oppenheimer et al. 1991; Wright and Flynn 2003). Using the spectral radiances in two bands the temperature of the cracks can be determined if the temperature of the homogenous crust is assumed (Rothery et al. 1988).

Monitoring must continue at Lascar. Our data suggest that Lascar is most likely at the end of an eruptive cycle. The dataset gives no indication of when, or if, a new cycle is going to begin at Lascar. Continued monitoring of the gas emissions and thermal flux is important in terms of predicting future activity at Lascar and for hazard mitigation. 


\section{Chapter 5}

An Estimation of Volcanic $\mathrm{SO}_{2}$ Emissions into the Troposphere from the Central and South American Volcanic Arcs 


\subsection{Introduction}

Passively degassing volcanoes are described as being in a non-eruptive state but persistently producing emissions of gasses such as sulfur dioxide $\left(\mathrm{SO}_{2}\right)$, water vapor $\left(\mathrm{H}_{2} \mathrm{O}\right)$ and carbon dioxide $\left(\mathrm{CO}_{2}\right)$ (McGonigle and Oppenheimer 2003). The quantification of gas emissions is a crucial part of interpreting volcanic activity. Gases control eruption style and are closely related to seismicity and ground deformation (Oppenheimer et al. 2003). Evaluating gas emissions is not only important for hazard mitigation but also from a climatological standpoint.

$\mathrm{SO}_{2}$ emitted from passively degassing volcanoes into the troposphere is climatologically significant (Graf et al. 1997; Horrocks et al. 2003). The extent of the impact on the climate from such $\mathrm{SO}_{2}$ emissions is poorly known especially in comparison to the effects of large injections of $\mathrm{SO}_{2}$ in to the stratosphere from explosive eruptions (Graf et al. 1997). Large quantities of $\mathrm{SO}_{2}$ introduced in to the stratosphere from immense eruptions such as El Chichon 1982, and Pinatubo, 1991, have been extensively studied and their impact on climate is better understood. $\mathrm{SO}_{2}$ emissions have an influence on the atmosphere by causing changes in its chemistry and radiation balance, both of which play a role in climate change (Robock 2000). $\mathrm{SO}_{2}$ in the atmosphere is converted into sulfate aerosols within several minutes to weeks (Oppenheimer 1998a; Carn et al. 2003; Rodriguez et al. 2008). The sulfate aerosols impact radiative transfer in the atmosphere, particularly in the stratosphere (Andres \& Kasgnoc 1998; Stenchikov et al. 1998). Aerosols scatter and absorb incoming shortwave radiation, as well as absorbing outgoing terrestrial longwave radiation. This warms the upper region of the atmosphere and cools the troposphere (Stenchikov et al. 1998). Additionally, sulfate aerosols promote heterogeneous chemical reactions that deplete the ozone layer (Prather 1992). 
Many volcanoes are notorious for their extensive periods of degassing not associated with an eruptive event, such as Mt Etna, Sicily and Kilauea, Hawaii. Such emissions form a substantial proportion of the contribution of volcanic gas to the atmosphere (Horrocks et al. 2003). In fact the yearly time-averaged magnitude of continuous emissions from passively degassing volcanoes exceeds that of those from sporadic eruptions such as Mt Pinatubo (Mather et al. 2003). In the troposphere, $\mathrm{SO}_{2}$ emissions and sulfate aerosol impact atmospheric radiation by absorbing and backscattering short wave radiation and affecting cloud cover (Mather et al. 2003). These emissions also have an impact on human health and the local environment such as killing crops and contaminating water supplies (Baxter et al. 1982; Allen et al. 2000; Delmelle et al. 2001).

Several studies have attempted to estimate the global contribution of volcanic $\mathrm{SO}_{2}$ to the atmosphere (Table 5.1). The estimates range from 1.5-50 million tons of $\mathrm{SO}_{2}$ per year and have been calculated using a variety of techniques such as ground based and satellite based remote sensing, melt inclusion data, estimations based on the volcanic explosivity index (VEI) (Schnetzler et al. 1997), lava effusion rates and plume size. 
Table 5.1

Previous estimates of global $\mathrm{SO}_{2}$ emissions from volcanoes and the technique used. The estimates include passive and eruptive emissions.

\begin{tabular}{|c|c|c|}
\hline Author & $\mathrm{Tg} / \mathbf{a}$ & Technique \\
\hline Kellogg et al. (1972) & 1.5 & $\begin{array}{l}\text { Calculated using the density of lava } \\
\text { and the volume erupted. It was } \\
\text { assumed that gas made up } 0.5 \% \text { wt } \\
\% \text { of the lava. }\end{array}$ \\
\hline Friend (1973) & 4.0 & $\begin{array}{l}\text { Friend used a similar technique to } \\
\text { Kellogg et al. (1972), by utilizing lava } \\
\text { volume, mass and gas content to } \\
\text { calculate a flux. }\end{array}$ \\
\hline Stoiber and Jepsen (1973) & $\overline{10.0}$ & $\begin{array}{l}\text { COSPEC measurements were taken } \\
\text { to calculate the volcanic flux from } \\
\text { volcanoes in Central America. This } \\
\text { result was then extrapolated to a } \\
\text { global estimation of } \mathrm{SO}_{2} \text {. }\end{array}$ \\
\hline Cadle (1975) & 7.5 & $\begin{array}{l}\text { Based on the calculation made by } \\
\text { Kellogg et al. (1972) but using a gas } \\
\text { content of } 2.5 \% \text { wt } \% \text {. }\end{array}$ \\
\hline LeGuern (1982) & $\overline{10.0}$ & $\begin{array}{l}\text { An estimate was based on lava } \\
\text { density, mass, and gas content. } \\
\text { Unlike previous studies viscosity was } \\
\text { taken in to consideration. }\end{array}$ \\
\hline $\begin{array}{l}\text { Berresheim and Jaeschke } \\
\text { (1983) }\end{array}$ & 15.0 & $\begin{array}{l}\text { Developed a classification scheme } \\
\text { based on volcanic activity and data in } \\
\text { the literature to determine a } \mathrm{SO}_{2} \\
\text { output. }\end{array}$ \\
\hline Stoiber et al. (1987) & 18.0 & $\begin{array}{l}\text { A questionnaire was used and sent to } \\
\text { other volcanologists. Questions were } \\
\text { related to plumes and their size. }\end{array}$ \\
\hline Lambert et al. (1988) & 50.0 & $\begin{array}{l}\text { Lead isotopes were used to calculate } \\
\mathrm{SO}_{2} \text { emissions. }\end{array}$ \\
\hline Andres and Kasgnoc (1997) & 13.0 & $\begin{array}{l}\text { Data collected by TOMS and } \\
\text { COSPEC. Also data from published } \\
\text { journals, personal communication and } \\
\text { conferences. }\end{array}$ \\
\hline Graf et al. (1997) & $28 \pm 12$ & Overview of previous work. \\
\hline Halmer et al. (2002) & $15-21$ & $\begin{array}{l}\text { TOMS, COSPEC and the } \\
\text { extrapolation of } \mathrm{SO}_{2} \text { data using the } \\
\text { Volcanic Sulfur Index (VSI) and the } \\
\text { Volcanic Explosivity Index (VEI). }\end{array}$ \\
\hline
\end{tabular}


This study will focus on determining an estimate of $\mathrm{SO}_{2}$ from passively degassing volcanoes (non-eruptive) in the Central and South American arc using satellite based remote sensing data from the Advanced Spaceborne Thermal Emission and Reflection Radiometer (ASTER) and the Ozone Monitoring Instrument (OMI). Central and South America are home to approximately 230 active volcanoes. Figure 5.1 is a location of the volcanoes in the Central and South American volcanic arcs. The volcanoes throughout the two arcs exhibit a range of styles of activity and magma compositions. Table 5.2 provides a brief description of many of the volcanoes in Central and South America that are investigated. 


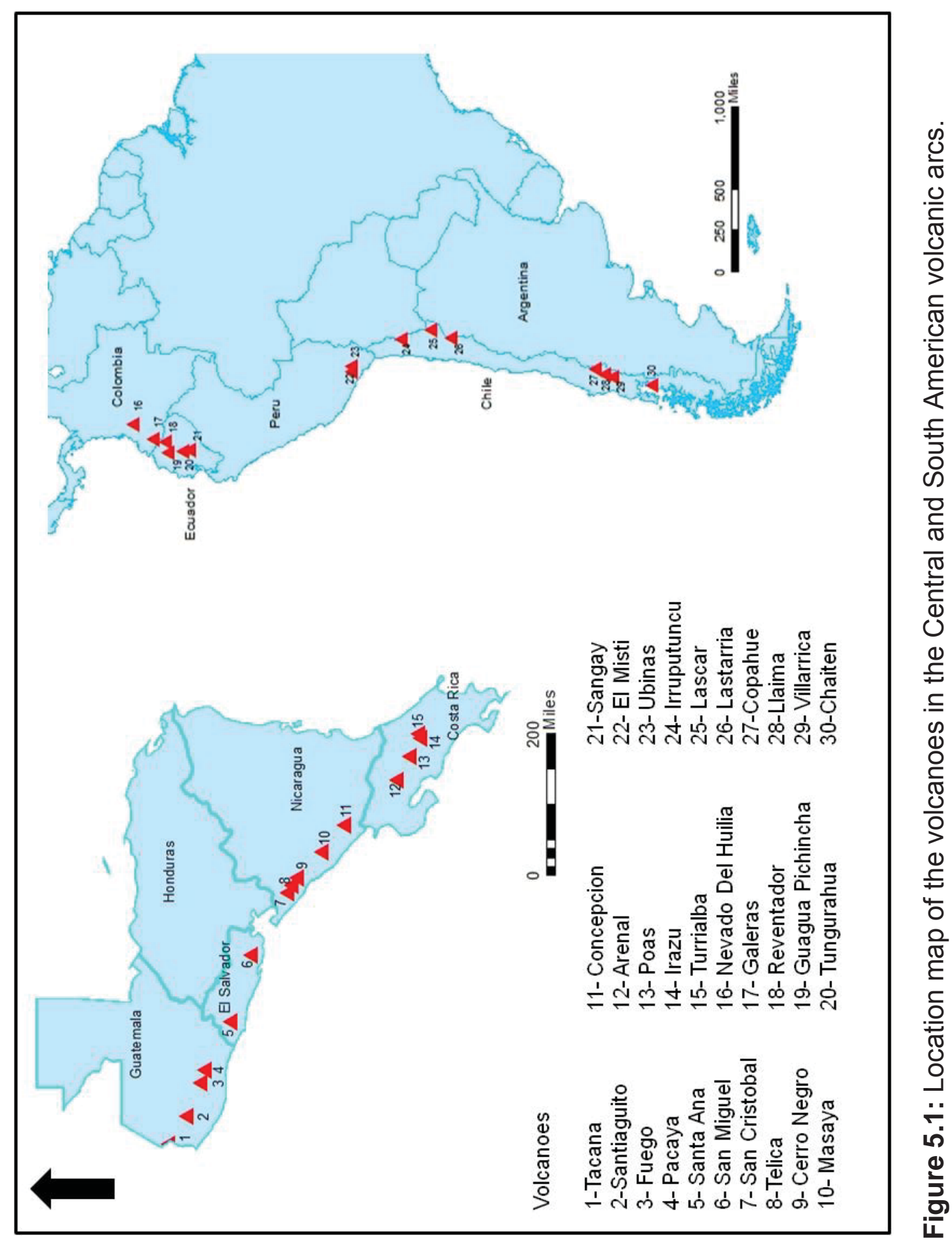




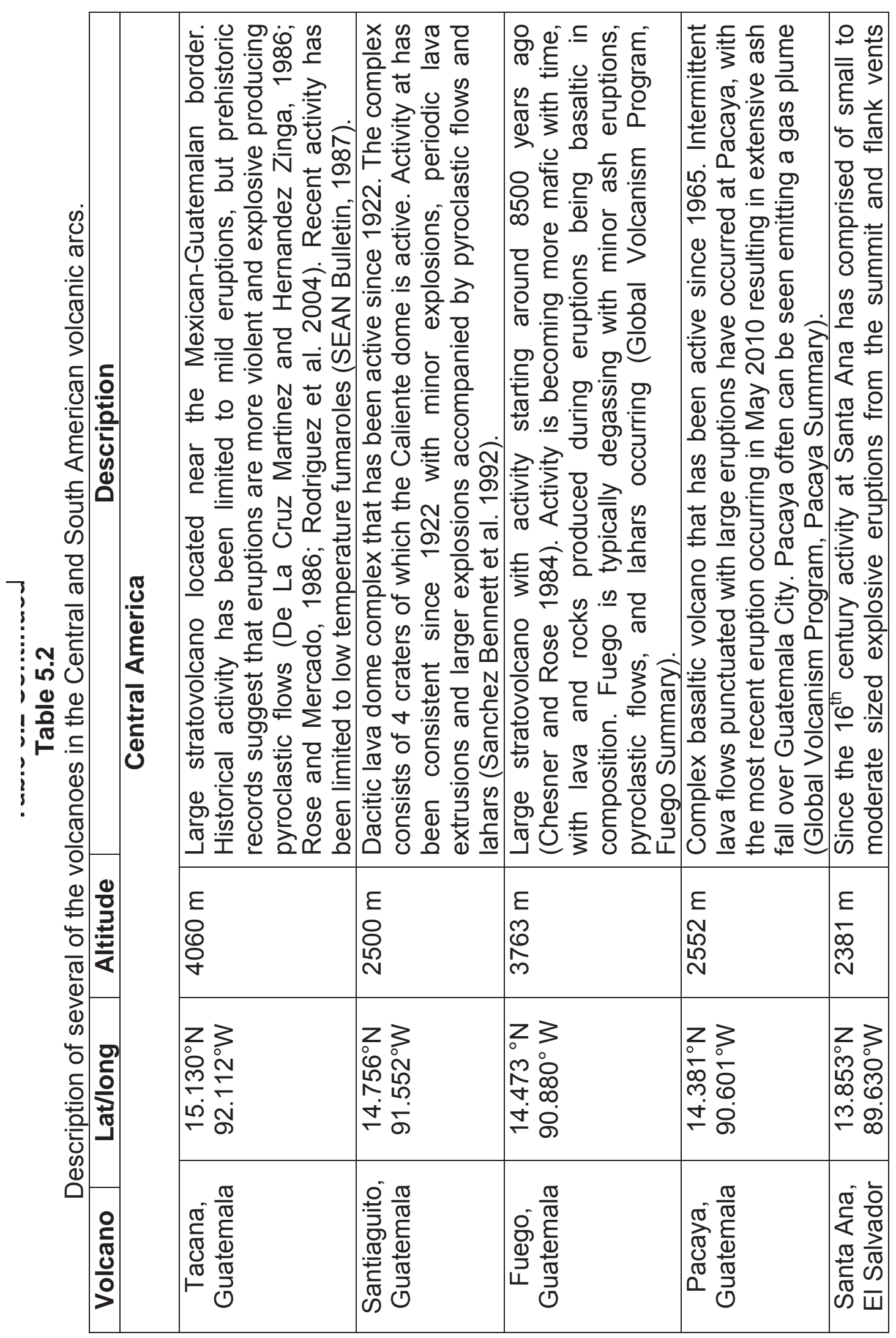




\begin{tabular}{|c|c|c|c|c|c|c|}
\hline 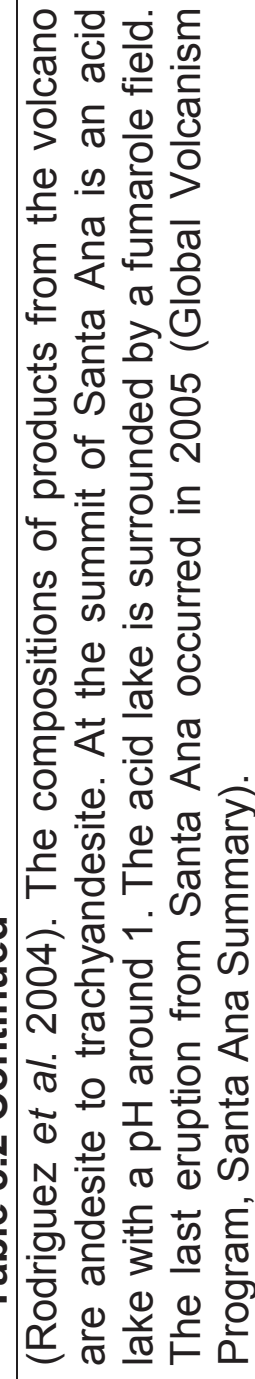 & 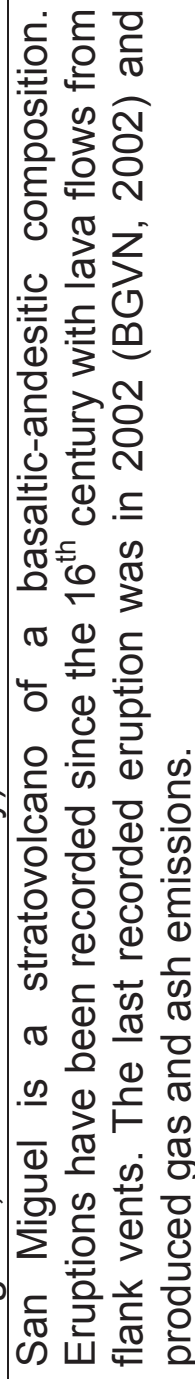 & 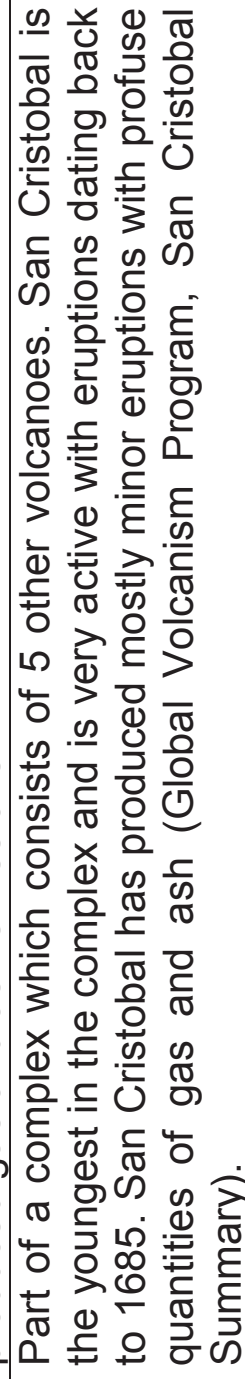 & 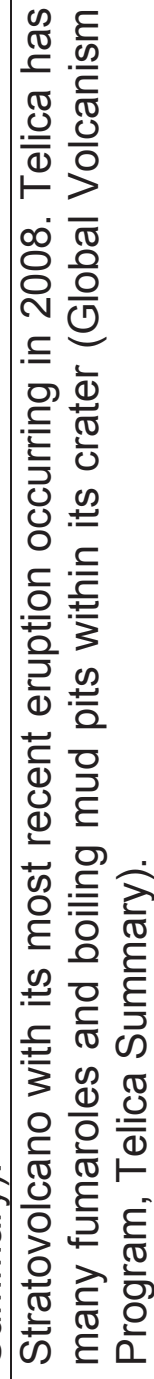 & 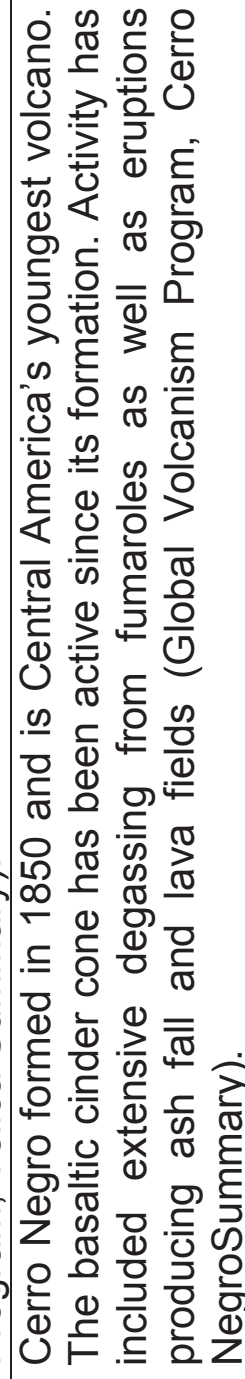 & 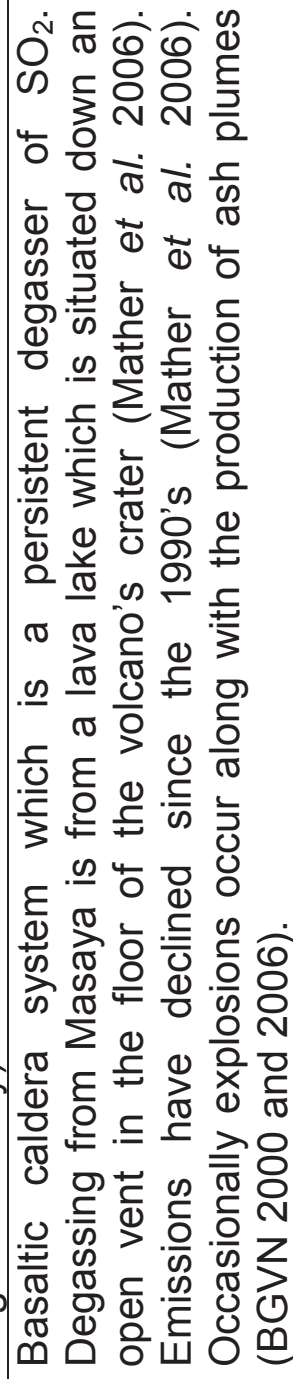 & 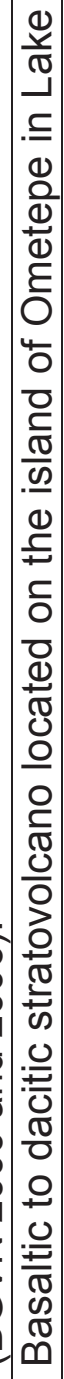 \\
\hline & $\begin{array}{l}\varepsilon \\
0 \\
m \\
\stackrel{N}{N}\end{array}$ & 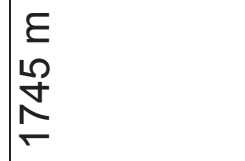 & 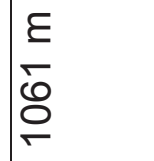 & $\begin{array}{l}\varepsilon \\
\infty \\
\stackrel{\Sigma}{N}\end{array}$ & $\begin{array}{l}\varepsilon \\
\text { E } \\
\text { లె }\end{array}$ & \\
\hline & 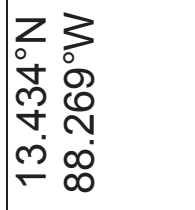 & 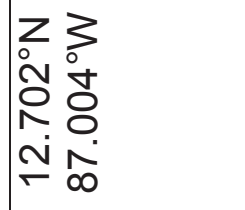 & 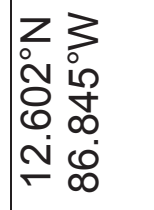 & 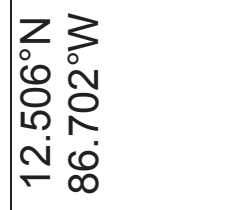 & $\begin{array}{ll}Z & 3 \\
0 & 0 \\
\dot{0} & 0 \\
\infty & 0 \\
0 & 0 \\
\check{\Gamma} & 0 \\
\leftarrow & \infty\end{array}$ & \\
\hline & 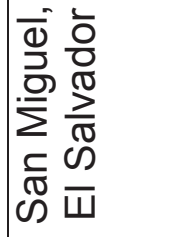 & 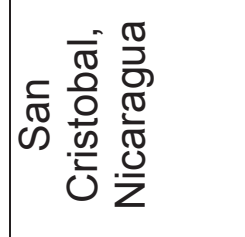 & 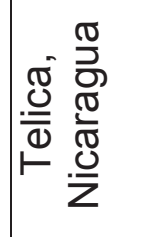 & 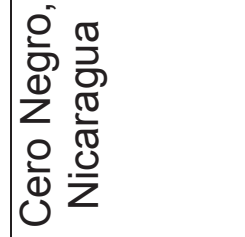 & 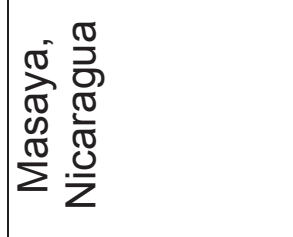 & \\
\hline
\end{tabular}




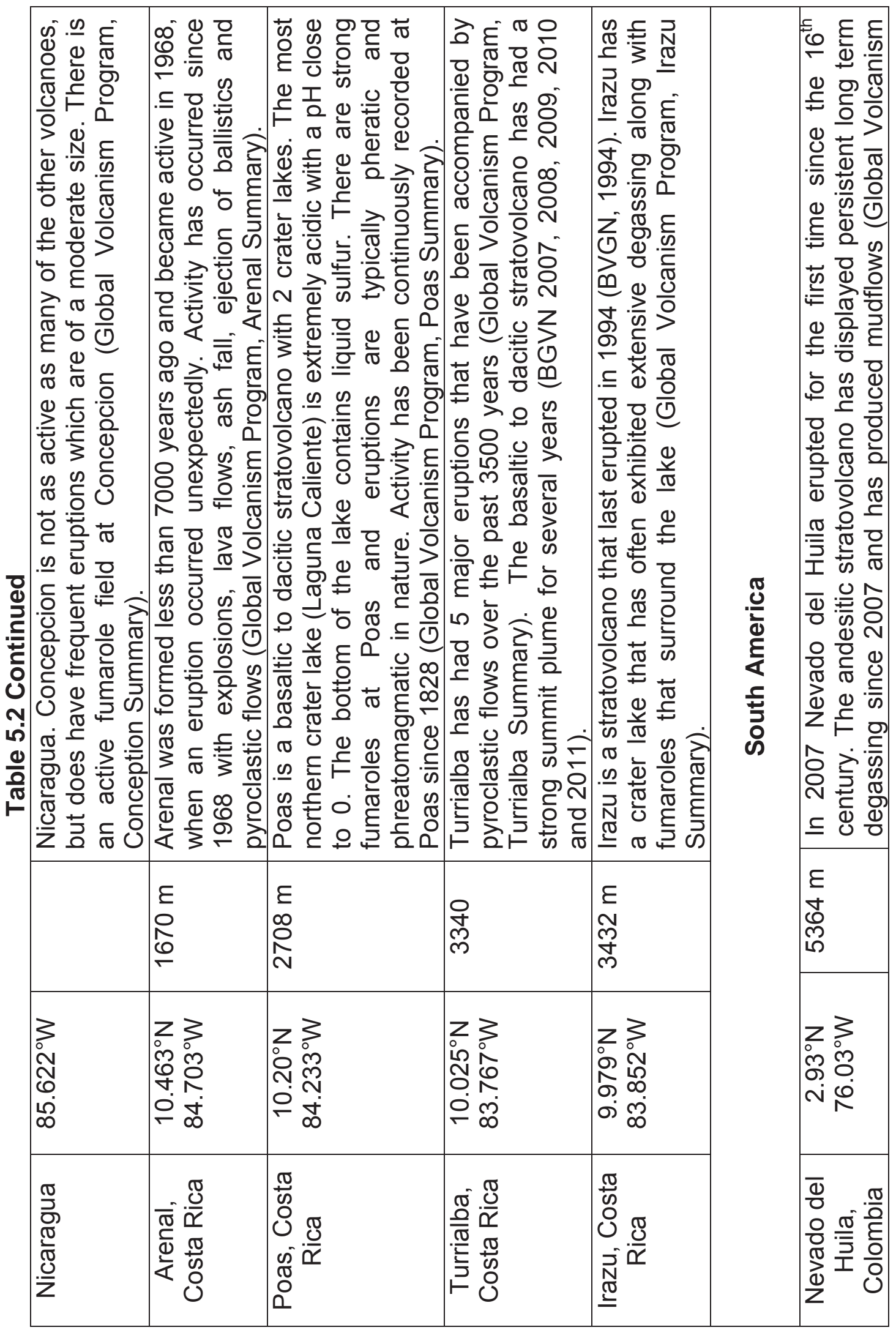




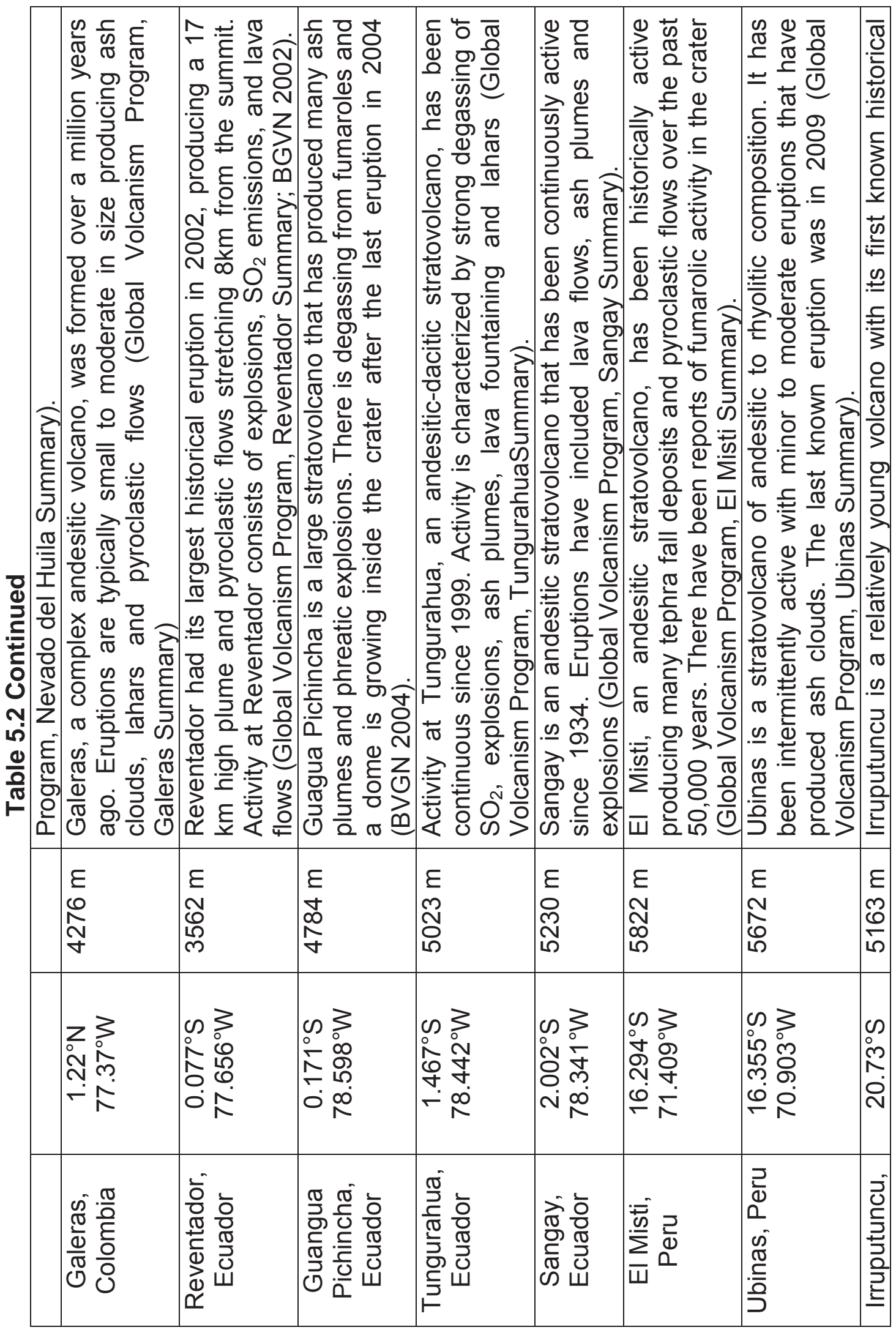




\begin{tabular}{|c|c|c|c|c|c|c|}
\hline 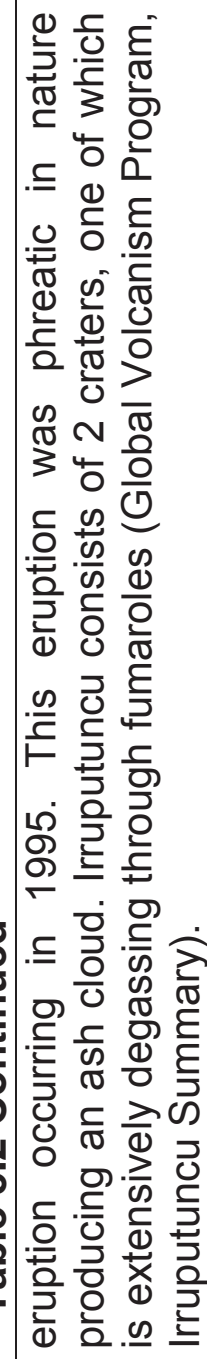 & 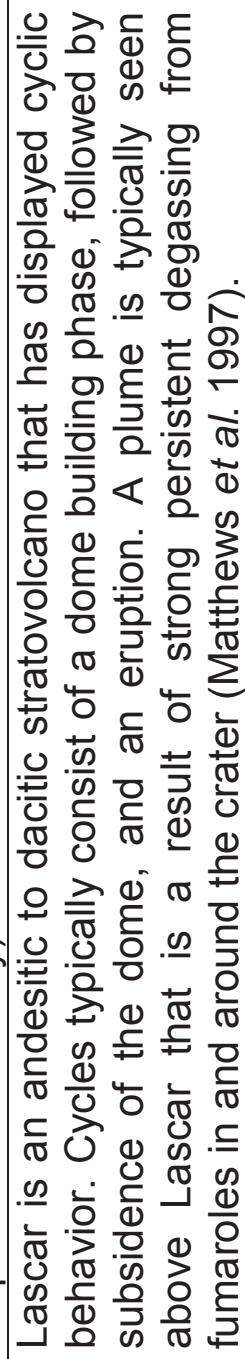 & 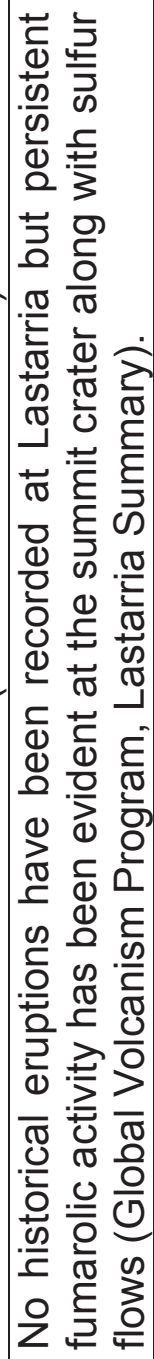 & 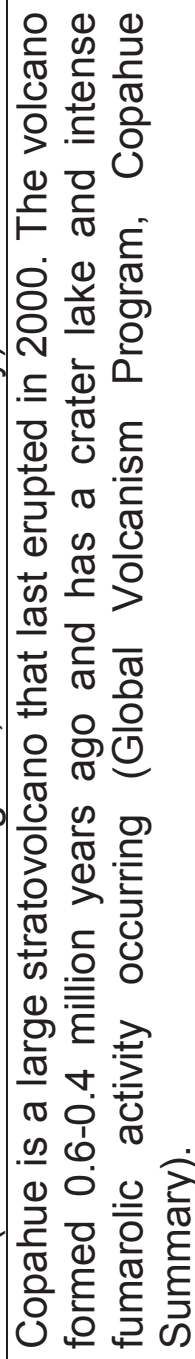 & 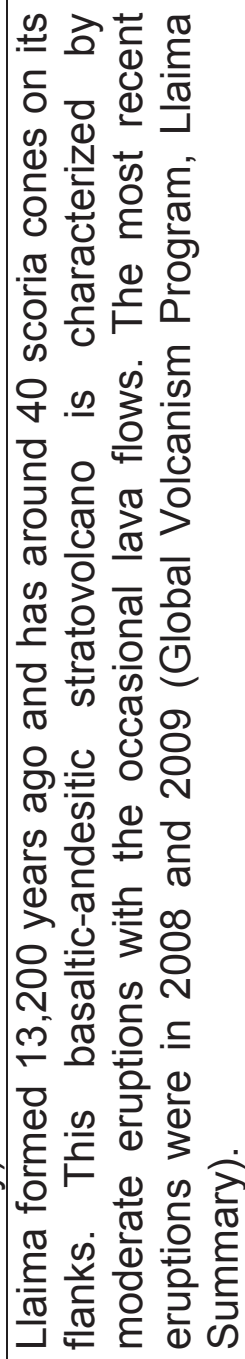 & 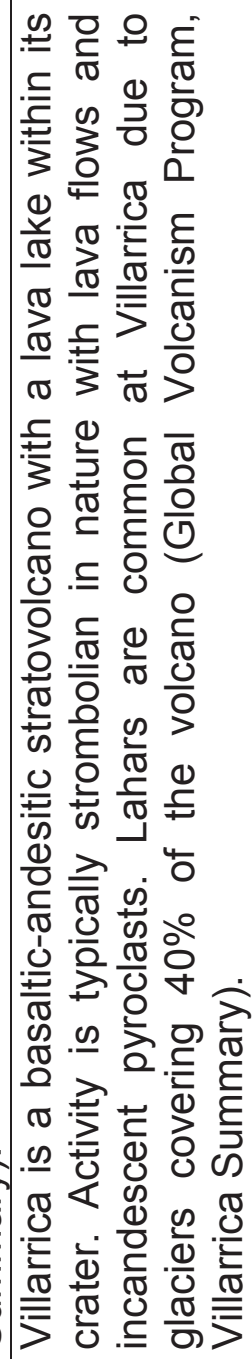 & 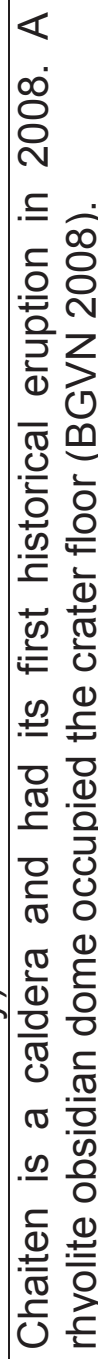 \\
\hline & 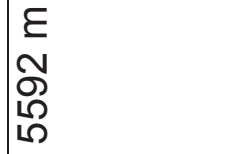 & $\begin{array}{l}\varepsilon \\
\hat{N} \\
\delta \\
6 \\
1\end{array}$ & $\begin{array}{l}\varepsilon \\
\hat{\sigma} \\
\text { م }\end{array}$ & $\begin{array}{l}E \\
\frac{E}{n} \\
\frac{n}{m}\end{array}$ & $\begin{array}{l}\varepsilon \\
\stackrel{E}{q} \\
\stackrel{N}{N}\end{array}$ & 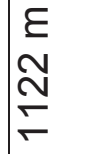 \\
\hline $\begin{array}{l}3 \\
0 \\
i n \\
\infty \\
\infty \\
0 \\
0\end{array}$ & 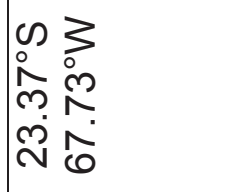 & 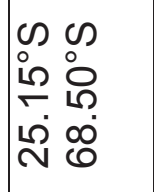 & 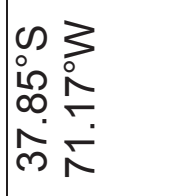 & 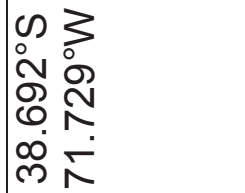 & 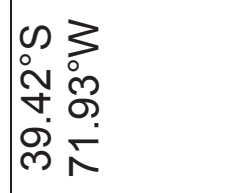 & 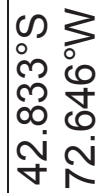 \\
\hline$\frac{0}{\frac{\mathbb{V}}{U}}$ & 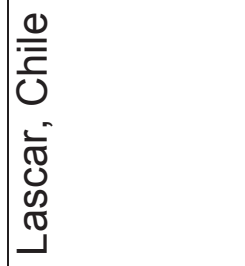 & 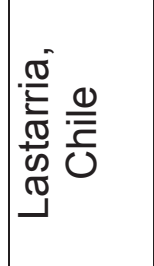 & 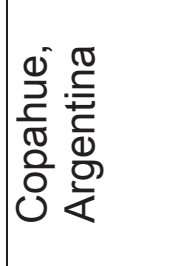 & 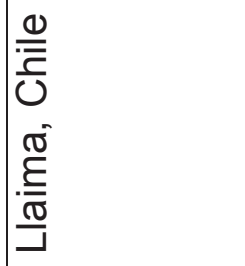 & 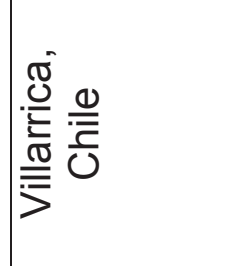 & 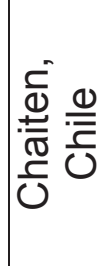 \\
\hline
\end{tabular}


Previous estimates of volcanic $\mathrm{SO}_{2}$ emissions have been made for both the Central and South American arcs as well as several of the individual countries that make up Central America. Halmer et al. (2002) estimated a flux of 0.6-0.8 Tg/a for the Central American arc and 2.1-3.6 Tg/a from the South American arc. These estimates are based on measurements from the Total Ozone Mapping Spectrometer (TOMS) and the Correlation Spectrometer (COSPEC). Where no COSPEC and TOMS data are available (prior to 1979), the volcanic sulfur dioxide index (VSI) was used to infer an $\mathrm{SO}_{2}$ flux from historical eruptions back to 1900. The VSI was developed using the average sulfur emissions from a volcano and the VEI of their eruptions (Schnetzler et al. 1997). Halmer et al. (2002) modified the VSI by a factor of two. This is because the original values being produced by the VSI were underestimating the average quantity of $\mathrm{SO}_{2}$ degassed from volcanoes. The new VSI was multiplied by the number of eruptions in a year to produce the annual global volcanic $\mathrm{SO}_{2}$ emissions from explosive eruptions for a given year. The calculated flux represents only a rough estimate since the majority of the gas measurements were assumed and predicted by the VEI and the VSI. This method only accounts for explosive eruptions not the average emissions during passive quiescent periods of activity.

Other studies have focused primarily on individual countries in Central America such as those carried out by Andres et al. (1993), Rodriguez et al. (2004) and Zimmer et al. (2004). Andres et al. (1993) estimated the output of volcanic $\mathrm{SO}_{2}$ from Guatemala. A flux of $0.18 \mathrm{Tg} / \mathrm{a}$ was estimated based on the three most active volcanoes in the country (Santiaguito, Fuego and Pacaya). The estimate was produced using an extensive COSPEC data set spanning a 20-year period. Rodriguez et al. (2004) expanded on this work estimating a volcanic flux of 0.28-1.45 Tg/a for volcanic emissions from Guatemala and El Salvador (from 1999-2002). This estimation was based on COSPEC measurements taken at six volcanoes throughout the two countries. Mather et al. (2006), added to this 
work by taking measurements at three volcanoes in Nicaragua, using Differential Optical Absorption Spectroscopy (DOAS). The results were combined with those obtained by Rodriguez et al. (2004) and data from Costa Rica (Zimmer et al. 2004) to produce an arc wide flux of $1.6 \mathrm{Tg} / \mathrm{a}$.

In South America several studies exist where individual volcanoes have been investigated. Andres et al. (1991) investigated emissions from Lascar and Lonquimay, Chile, in November 1989. Measurements were taken with a COSPEC and produced average fluxes of $2300 \pm 1120 \mathrm{t} \mathrm{d}^{-1}$ for Lascar and $2380+/-2720 \mathrm{t} \mathrm{d}^{-1}$ for Lonquimay. Witter et al. (2004) quantified emissions from Villarrica, Chile, in 2000 and 2001, using a COSPEC. An average of $260+/-170$ $\mathrm{t} \mathrm{d}^{-1}$ was reported in 2000, and an average of $460 \pm 260 \mathrm{t} \mathrm{d}^{-1}$ was reported in 2001.

$\mathrm{SO}_{2}$ emissions from Tungurahua, Ecuador, measured between August 1999 and October 2006 were presented by Arellano et al. (2008). An average emission rate of $1458 \mathrm{t} \mathrm{d}^{-1}$ was recorded, with emissions ranging between $100 \mathrm{t}$ $\mathrm{d}^{-1}$ and $35,000 \mathrm{t} \mathrm{d}^{-1}$ during the study period. Arellano et al. (2008) also calculated the average emission rate during periods of passive degassing and explosive activity at Tungurahua, with fluxes of $1391 \pm 1697 \mathrm{t} \mathrm{d}^{-1}$ and $2433 \pm$ $4623 \mathrm{t} \mathrm{d}^{-1}$ respectively. The total $\mathrm{SO}_{2}$ emissions to the atmosphere over the study period were $1.91 \mathrm{Tg}$. Carn et al. (2008) investigated the use of OMI to quantify emissions from passively degassing volcanoes in Ecuador, and Galeras in Colombia. The study concluded that the volcanoes in Ecuador along with Galeras, contributed $1.16 \mathrm{Tg}$ of $\mathrm{SO}_{2}$ to the troposphere over a 2-year period.

In this study ASTER was the primary sensor used due to its ability to detect $\mathrm{SO}_{2}$ in the thermal infrared at its $8.6 \mu \mathrm{m}$ absorption feature. With its high spatial resolution (90 $\mathrm{m}$ in the TIR), ASTER is beneficial for looking at plumes of low 
$\mathrm{SO}_{2}$ column abundance in the lower troposphere such as those emitted from Pacaya, Masaya, and Lascar. The detection limits of ASTER have been explored and have shown that the sensor can easily detect emissions from sources in excess of $\sim 1000 \mathrm{t} \mathrm{d}^{-1}$ (Henney 2006). Forward modeling has indicated that as little as $\sim 500 \mathrm{t} \mathrm{d}^{-1}$ can be detected from a plume at $4 \mathrm{~km}$ altitude in a tropical (high humidity) environment (Henney 2006). With ASTER operating in the TIR meteorological cloud cover is problematic. ASTER cannot detect $\mathrm{SO}_{2}$ emissions when a plume is obscured by clouds. A large portion of the volcanoes in this study are located in regions where cloud cover is abundant. It was expected that ASTER would have difficulty viewing and detecting any plumes in areas such as Colombia and Ecuador, where cloud cover is thick and often obscures many of the volcanoes and their plumes. To get around this issue, we use OMI to look at emissions at volcanoes where ASTER was capable.

OMI operates in the ultraviolet (UV) part of the spectrum and is able to detect emissions in the presence of clouds. Even though OMI can detect $\mathrm{SO}_{2}$ in cloudy regions, it should be noted that clouds also affect UV measurements. OMI will under- or over-estimate the column amount of $\mathrm{SO}_{2}$ detected depending on the location of the plume in relation to the cloud cover (Krotkov et al. 2006). OMI, with its greater temporal resolution than ASTER, provides more opportunities to view volcanic plumes. This is important for providing continuous data. It is expected that these instruments will aid in improving previous estimates. With their ability to detect smaller column amounts of $\mathrm{SO}_{2}$ the number of volcanoes that can be investigated remotely will increase. In addition to the remotely sensed data, a thorough literature review of published data was used to supplement our data. This provides a more complete dataset and makes this estimate as precise as possible. This study provides an opportunity to explore and test the capability of the ASTER and OMI sensors at detecting $\mathrm{SO}_{2}$, at a variety of volcanoes. Such information is important in terms of knowing what 
sensors can and cannot detect under various conditions such as plume altitude, cloud cover and $\mathrm{SO}_{2}$ column abundance. 


\subsection{Methodology}

Due to the number of volcanoes in Central and South America it is impractical to collect every ASTER and OMI image for every volcano over a 10 year time span and process the images for $\mathrm{SO}_{2}$. Using the Global Volcanism Program's (http://www.volcano.si.edu) extensive database, and reports on all the volcanoes in South and Central America, the number of volcanoes in the study were narrowed down from 234 to 90 . These 90 volcanoes were narrowed down even further by reading their activity reports to determine if they were a likely source of $\mathrm{SO}_{2}$. ASTER images were processed from 2000 through early 2011. This period was chosen for 2 reasons. First, ASTER imagery is only available from around 2000 onwards due to the sensor being launched in December 1999 onboard the Terra platform. Second, this time scale is short enough to reflect long term degassing trends while being long enough to remove interannual variability (Halmer et al. 2000).

Over 6000 L1B ASTER images were downloaded (from http://reverb.echo.nasa.gov/reverb/). Not all of these images were processed for $\mathrm{SO}_{2}$. The images are prescreened to determine if any $\mathrm{SO}_{2}$ plumes can be quantified using the IDL MAP_SO2 algorithm (refer to Introduction chapter for details on algorithm). Figure 5.2 is a flowchart summarizing the steps involved in pre-screening the ASTER images. To detect $\mathrm{SO}_{2}$ from ASTER the TIR channels are used. However, meteorological clouds are a problem in TIR imagery and obscure $\mathrm{SO}_{2}$. Any images that contain too many clouds, obscuring the plume from the target volcano, are disregarded. The images are screened for clouds (and ash) by viewing them in the VNIR and TIR. Occasionally an $\mathrm{SO}_{2}$ plume cannot be seen in the NVIR or TIR image, especially if the plume is weak or the plume boundaries are uncertain, blending into the remainder of the image. To try and highlight any possible plumes in an image, a principal component analysis (PCA) and de-correlation stretch (DCS) are carried out (refer to Chapter 3 for details on these enhancement techniques). Figure 5.3 
illustrates a plume in the TIR that has been enhanced using a PCA and a DCS. After all the images were prescreened, approximately 2500 were then further processed to quantify any $\mathrm{SO}_{2}$ that might be present.

OMI and ground-based data are used to supplement the ASTER data and fill in the gaps where data are poor or not available. OMI data are available from 2004 onwards and were used to look at emissions primarily from volcanoes in Ecuador and Colombia. OMI was also used to detect $\mathrm{SO}_{2}$ emissions from Fuego, Pacaya, Masaya, Turrialba, Ubinas, El Misti, Lascar, Lastarria, and Villarrica. Daily OMI images are available to browse through the Global Sulfur Dioxide Monitoring home page (http://so2.gsfc.nasa.gov/).

$\mathrm{SO}_{2}$ estimated from $\mathrm{OMI}$ data are reported in mass (kilotons) for the entire plume, not as a flux in $\mathrm{t} \mathrm{d}^{-1}$. In this study, to make the OMI data more comparable to the ASTER and published data it was converted into tons by multiplying the mass by 1000 . This also makes it easier to calculate an average flux for each volcano and the volcanic arcs as a whole. Using the mass of the entire plume will add error to the estimate and has some problems associated with it. This will be discussed further below in the discussion (section 5.4.2). Santa Ana, Turrialba, Lascar, Lastarria, and Ubinas volcanoes were the exception, where the mass was converted to a daily flux in $\mathrm{t} \mathrm{d}^{-1}$ by taking a transect across the plume close to the vent. Not all the volcanoes had a daily flux calculated by taking a transect across the plume due to time constraints.

The ASTER, OMI, and ground-based (published data) were combined to determine an average flux for each volcano and a flux for each of the volcanic arcs. Details of the $\mathrm{OMI}$ and ASTER algorithms used to quantify $\mathrm{SO}_{2}$ are described in the introduction (Chapter 1). 


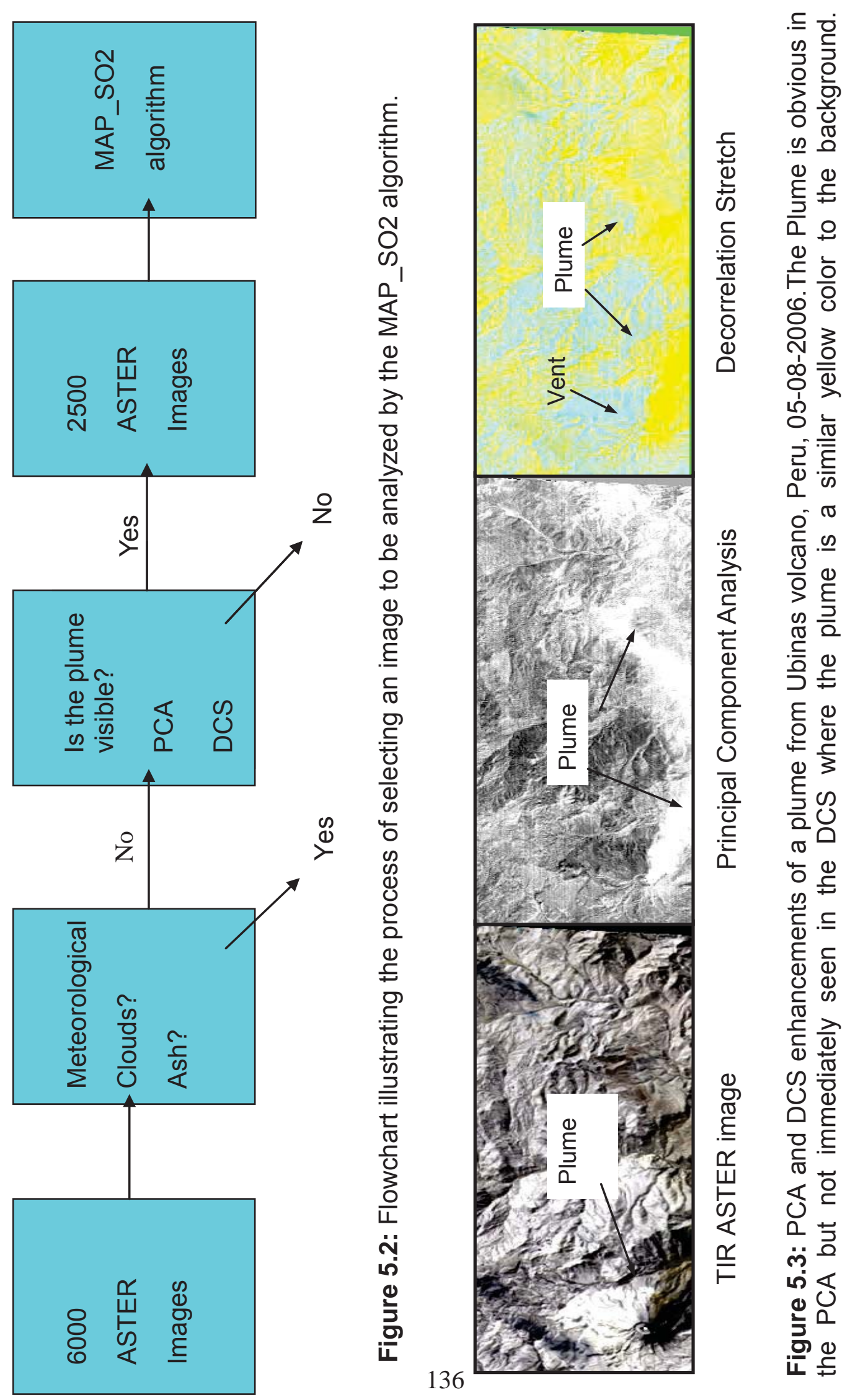




\subsection{Results}

\subsubsection{Central American Arc}

It is estimated that the volcanoes in the Central American arc contribute $3.4 \mathrm{Tg}$ of $\mathrm{SO}_{2}$ to the atmosphere on average each year between 2000 and 2010. Table 5.3 shows the breakdown of the emissions from each volcano and country. This estimation was calculated using ASTER, OMI, and ground based fluxes obtained from a review of published data. In the Central American arc the majority of the estimation is based on ASTER and ground-based data. OMI provided extensive data sets for Turrialba volcano, Costa Rica, and Fuego and Pacaya volcanoes, Guatemala. Figure 5.4 illustrates some of the images of the plume at Turrialba collected by OMI. OMI was not used to investigate other volcanoes in the Central American arc such as Masaya and San Cristobal due to time constraints. Due to the number of $\mathrm{SO}_{2}$ maps produced by ASTER it is impossible to include them all within this work. Therefore, examples from each volcano are located in Figure 5.5 and Table 5.6.

To calculate an average for a volcano, the fluxes were added together and then divided by the number of measurements (days) that were acquired. Where there were multiple measurements for a single day, an average was calculated for that day before being used to calculate an annual average for that particular volcano. Appendix 3 contains a compilation of all the measurements used to calculate the average flux for each volcano in this study. The appendix contains the method and date the flux was obtained, along with the source of the data. 
Table 5.3

Estimate of $\mathrm{SO}_{2}$ emissions from volcanoes in the Central American Volcanic arc (2000-2010). The estimate is broken down in to individual volcanoes and by country.

\begin{tabular}{|c|c|}
\hline Guatemala & Flux $\left(\mathrm{t} \mathrm{d}^{-1}\right)$ (baseline average) \\
\hline Santiaguito & 123.5 \\
\hline Fuego & 354.9 \\
\hline Pacaya & 1382.2 \\
\hline Tacana & 30.0 \\
\hline total & $0.7(\mathrm{Tg} / \mathrm{a})$ \\
\hline \multicolumn{2}{|l|}{ El Salvador } \\
\hline Santa Ana & 1783.6 \\
\hline San Miguel & 215.0 \\
\hline total & 0.8 \\
\hline \multicolumn{2}{|l|}{ Nicaragua } \\
\hline Masaya & 924.6 \\
\hline San Cristobal & 2141.5 \\
\hline Telica & 354.0 \\
\hline total & 1.2 \\
\hline \multicolumn{2}{|l|}{ Costa Rica } \\
\hline Poas & 108.8 \\
\hline Turrialba & 1638.7 \\
\hline Arenal & 215.8 \\
\hline total & 0.7 \\
\hline Arc total & $\begin{array}{l}9067+/-2720(10,986)^{1^{*}} \mathrm{t} \mathrm{d}^{-1} \\
\text { or } 3.4+/-1.02(4.0)^{2^{\star}} \mathrm{Tg} / \mathrm{a}\end{array}$ \\
\hline
\end{tabular}

${ }^{2 *}$ is the total flux corrected for unmeasured $\mathrm{SO}_{2}$ emissions using the power law proposed by Brantley and Koepenick (1995). 

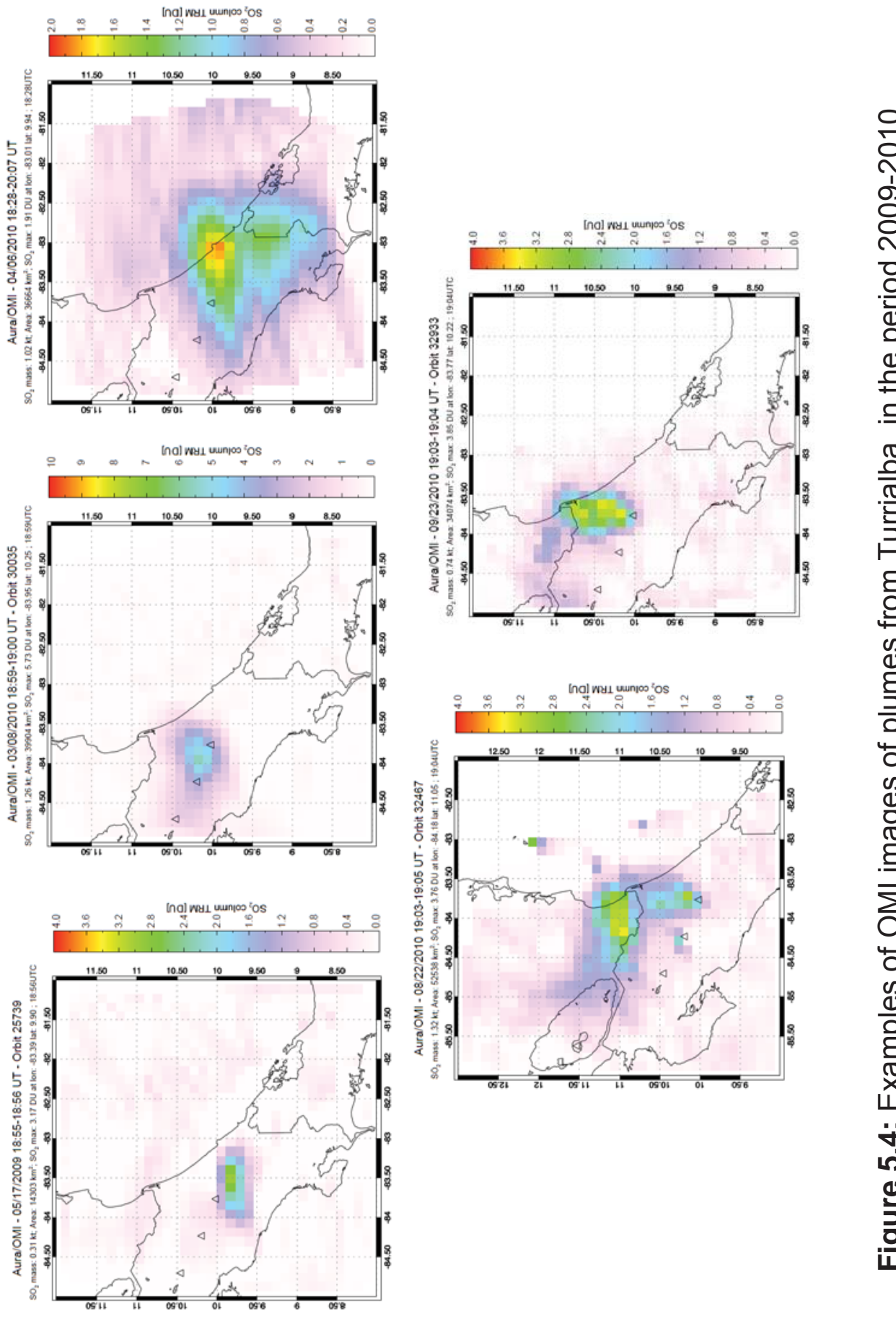

은

क

흥

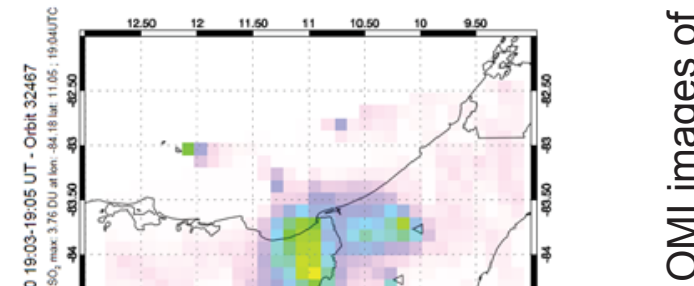




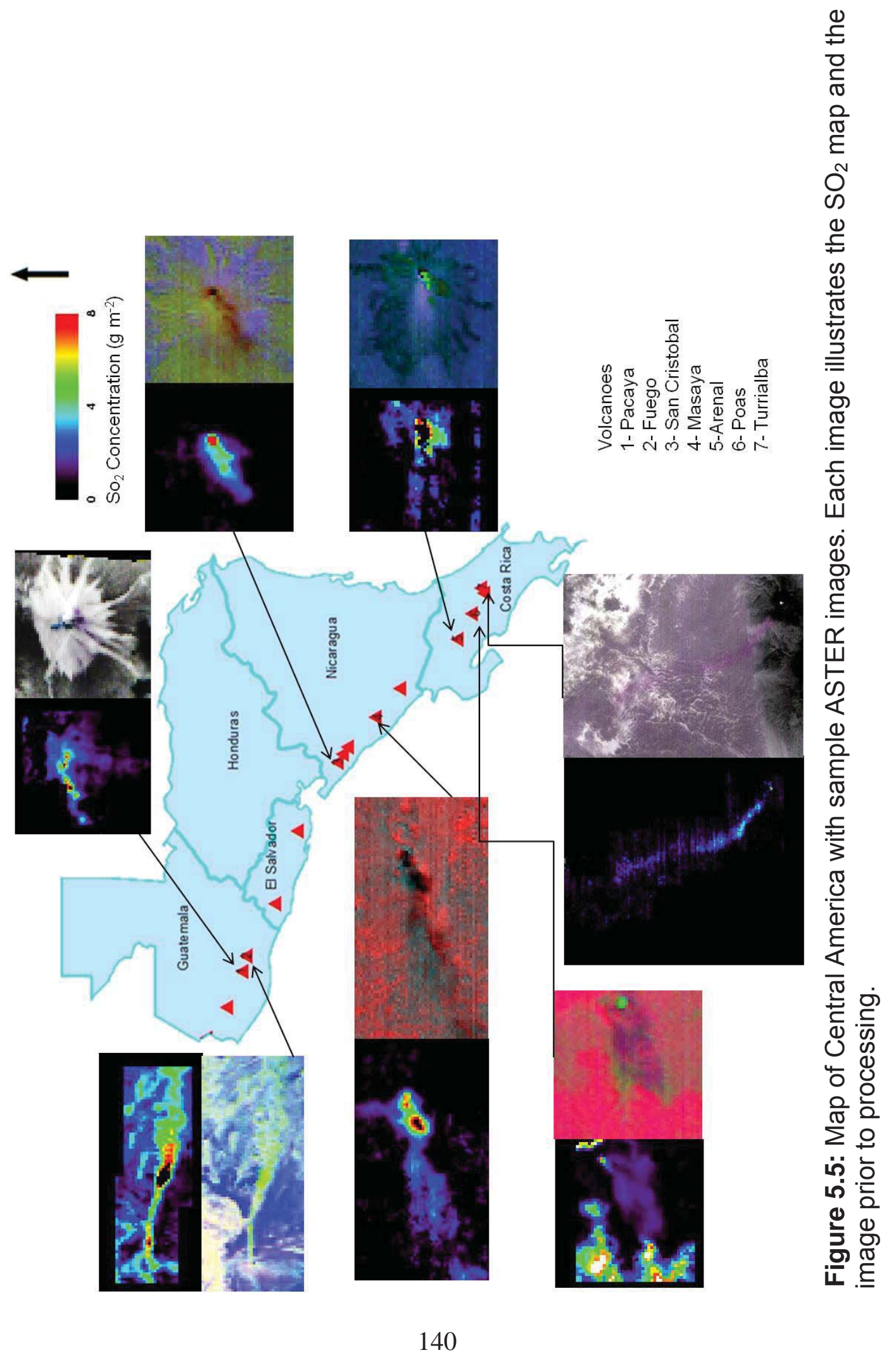




\subsubsection{Guatemala}

An overall flux of $0.7 \mathrm{Tg} / \mathrm{a}$ was estimated for Guatemala. Emissions were detected from 4 volcanoes, Pacaya, Fuego, Santiaguito, and Tacana. ASTER was only able to detect $\mathrm{SO}_{2}$ emissions from Pacaya volcano between 2000 and 2003. The plumes quantified during this time period were typically in excess of $1000 \mathrm{t} \mathrm{d}^{-1}$. Using COSPEC data (Rodriguez et al. 2004), UV Camera data (Dalton et al. 2010) and ASTER data, an average of $1382 \mathrm{t} \mathrm{d}^{-1}$ was calculated for Pacaya between 2000 and 2003.

Previous averages calculated for Pacaya include that by Andres et al. (1993) with a flux of $260 \mathrm{t} \mathrm{d}^{-1}$, and Rodriguez et al. (2004) with a flux of $1350 \mathrm{t} \mathrm{d}^{-1}$. Our average is well in excess of that calculated by Andres et al. (1993), but similar to that produced by Rodriguez et al. (2004). With no $\mathrm{SO}_{2}$ being detected in any of the 60 ASTER images available after 2003, it cannot be assumed that Pacaya is not emitting any $\mathrm{SO}_{2}$ (or other gases). Reports by the BGVN from 2005-2009 describe Pacaya as being continually active after 2003, producing lava flows and gas/steam emissions typically free of ash (BGVN 2005; 2008; and 2009). Work carried out by Dalton et al. (2010), using the UV camera in January 2008, illustrated that the $\mathrm{SO}_{2}$ flux at Pacaya was highly variable. Over a 2-hour time period emissions ranged from a maximum of $200 \mathrm{t} \mathrm{d}^{-1}$ to a minimum of $41 \mathrm{t} \mathrm{d}^{-1}$. Such data suggests that when Pacaya is active emissions are well over $1000 \mathrm{t} \mathrm{d}^{-1}$ and easy to detect by ASTER, but during quiet phases emissions are below $200 \mathrm{t} \mathrm{d}^{-1}$ and undetectable. This would indicate that an average of over $1000 \mathrm{t} \mathrm{d}^{-1}$ is too high for Pacaya and that the average is closer to the baseline average $\left(260 \mathrm{t} \mathrm{d}^{-1}\right)$ calculated by Andres et al. (1993). If Pacaya's daily flux is closer to $260 \mathrm{t} \mathrm{d}^{-1}$, then the average yearly flux emitted from Guatemala and the Central American volcanic arc is reduced by $0.5 \mathrm{Tg} / \mathrm{a}$.

ASTER did not detect plumes at Santiaguito or Tacana and only 1 ASTER image identified a plume at Fuego (1 March 2002). Published ground based 
data were used to calculate averages for these volcanoes. Rodriguez et al. (2004) provided data for Tacana. Sources of data for Santiaguito are BVGN (2000), Rodriguez et al. (2004) and Holland et al. (2011). Sources of data for Fuego are BGVN (2002) and Rodriguez et al. (2004).

Fluxes at Santiaguito are very low, and it was not certain that ASTER would be able to detect any $\mathrm{SO}_{2}$. Work carried out by Holland et al. (2011), in early 2009, utilizing the UV camera, indicated a maximum flux of $285 \mathrm{t} \mathrm{d}^{-1}$ and a minimum of $38 \mathrm{t} \mathrm{d}^{-1}$ at Santiaguito. Typically, emissions from Santiaguito are weak. It is only when there is a minor explosion that the emission rate increases toward the maximum reported by Holland et al. (2011). During the explosions, when the maximum $\mathrm{SO}_{2}$ flux is reported, ash is also emitted. Ash will have an impact and add error to the UV camera measurements. When $\mathrm{SO}_{2}$ emissions are quantified during a period of repose when no ash is present, an error of $\pm 18.7 \%$ is applied to the data. During the explosions, when the maximum $\mathrm{SO}_{2}$ flux is reported an ash correction procedure is needed. Data collected during explosions have an error of $29.6 \%$ due to the ash correction procedure (Holland et al. 2011).

OMI was capable of detecting plumes in the Pacaya/Fuego region. It is unknown what the source of the plume is due to the close proximity of the two volcanoes. An average of $50 \mathrm{t} \mathrm{d}^{-1}$ from the two volcanoes combined was calculated from 2004-2010.

\subsubsection{El Salvador}

An overall $\mathrm{SO}_{2}$ flux of $0.8 \mathrm{Tg} / \mathrm{a}$ was estimated for El Salvador. Santa Ana is in a constant state of degassing and 26 measurements were obtained by groundbased instruments between 2001 and 2006 (BGVN 2001; BGVN 2005; Rodriguez et al. 2004; and Barrancos et al. 2008). The average calculated from these measurements is $1783 \mathrm{t} \mathrm{d}^{-1}$. Due to the average ground based flux being 
high and in excess of $1000 \mathrm{t} \mathrm{d}^{-1}$, it was assumed that many ASTER images would be capable of quantifying plumes from Santa Ana. However, many of the 77 ASTER images acquired were too cloudy to see the vent at Santa Ana. Santa Ana went through an eruptive episode in 2005. Fluxes in the years prior to 2005 are less than $400 \mathrm{t} \mathrm{d}^{-1}$, especially in 2002 when fluxes are barely above $100 \mathrm{t} \mathrm{d}^{-1}$ (BVGN 2001; Rodriguez et al. 2004; Barrancos et al. 2008). During the eruptive episode fluxes were in excess of $3200 \mathrm{t} \mathrm{d}^{-1}$ before slowly returning to less than $400 \mathrm{t} \mathrm{d}^{-1}$ in early 2006 (Barrancos et al. 2008). ASTER is not capable of detecting emissions of less than $100 \mathrm{t} \mathrm{d}^{-1}$ and will be working close to its detection limits to detect emissions around $400 \mathrm{t} \mathrm{d}^{-1}$. During the eruptive episode, only 4 ASTER images were available. These images were all cloudy and no plume could be detected. OMI data were investigated over the eruptive episode (2005) and detected $\mathrm{SO}_{2}$ in images from 25 August 2005 and 1 October 2005, with fluxes of $713 \mathrm{t} \mathrm{d}^{-1}$ and $12,537 \mathrm{t} \mathrm{d}^{-1}$ respectively (Figure 5.6).

No $\mathrm{SO}_{2}$ was detected in ASTER or OMI imagery of San Miguel. COSPEC measurements were obtained in January 2002 (BGVN 2002; Rodriguez et al. 2004) and October 2006 (BGVN 2007) to provide an average flux of $215 \mathrm{t} \mathrm{d}^{-1}$. 


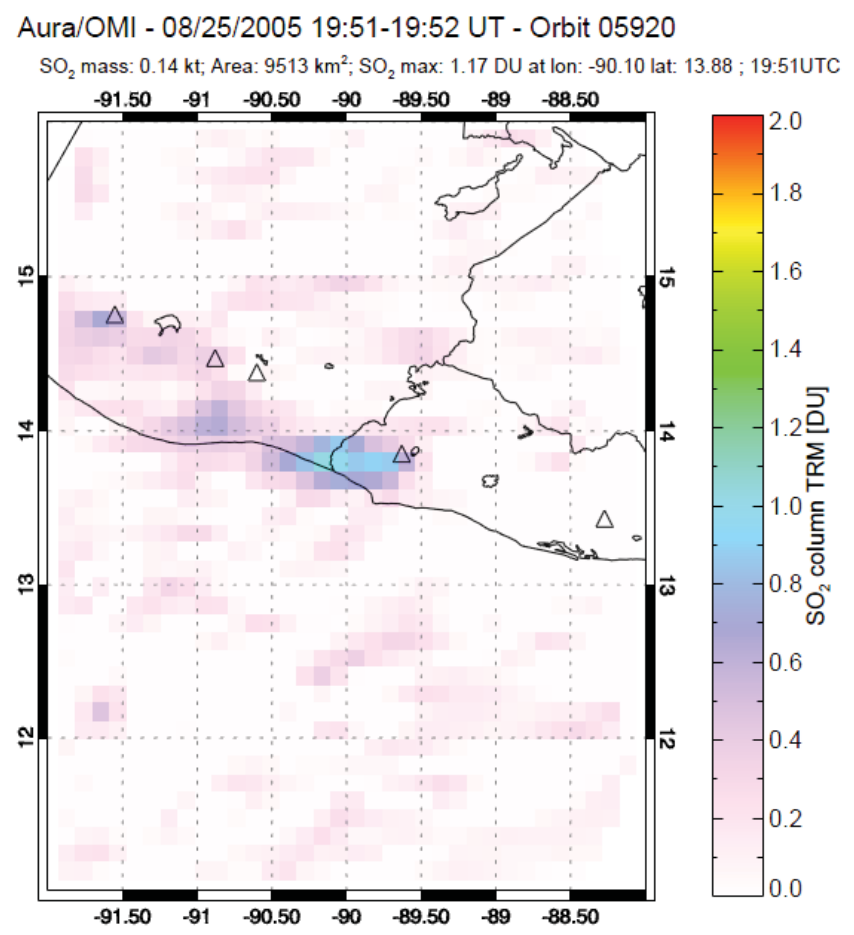

Aura/OMI - 10/01/2005 20:09-20:10 UT - Orbit 06459

$\mathrm{SO}_{2}$ mass: $11.80 \mathrm{kt}$; Area: $71779 \mathrm{~km}^{2} ; \mathrm{SO}_{2}$ max: $23.47 \mathrm{DU}$ at lon: -90.54 lat: $13.75 ; 20: 10 \mathrm{UTC}$

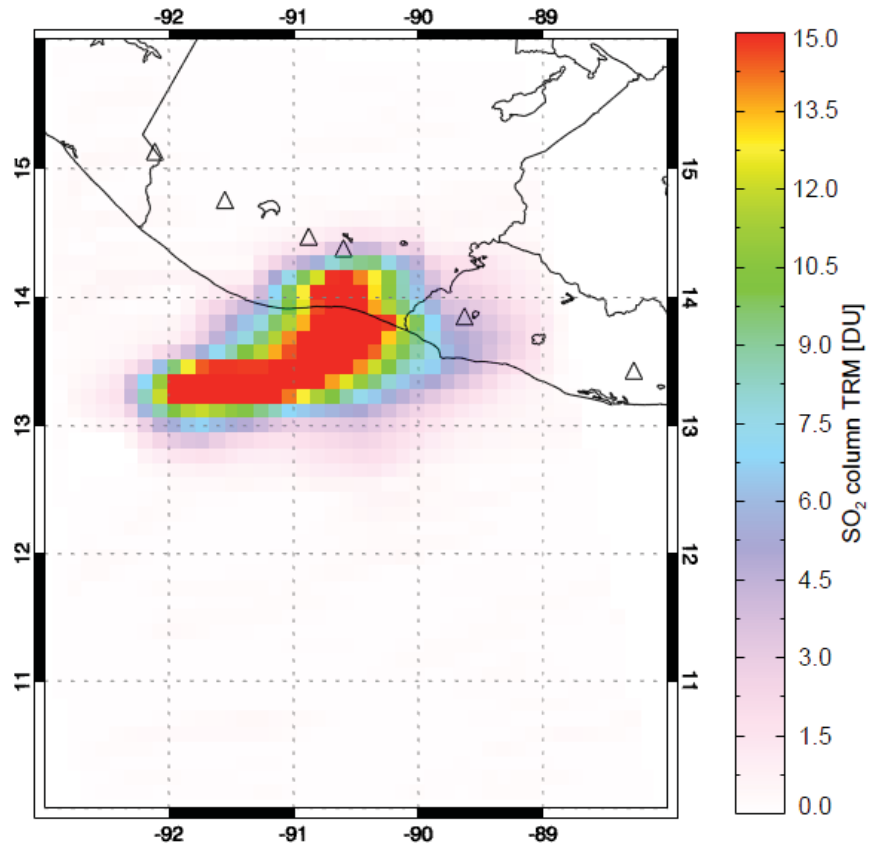

Figure 5.6: OMI images of plumes produced during the 2005 eruptive episode at Santa Ana. Images are from 25 August 2005 and 1 October 2005 with fluxes of $713 \mathrm{t} \mathrm{d}^{-1}$ and $12,537 \mathrm{t} \mathrm{d}^{-1}$ respectively. 


\subsubsection{Nicaragua}

It is estimated that $1.2 \mathrm{Tg} / \mathrm{a}$ of $\mathrm{SO}_{2}$ were emitted from volcanoes in Nicaragua. A total of 5 volcanoes were investigated in Nicaragua with Masaya and San Cristobal both having emissions detected by ASTER. It was uncertain if ASTER (or OMI) would be able to detect and quantify $\mathrm{SO}_{2}$ from Masaya due to its very low altitude $(635 \mathrm{~m})$ and a plume that often flows down the flanks of the volcano hugging the ground (Delmelle et al. 2002). Low altitude plumes are more difficult for ASTER to detect. This is because of the reduced temperature contrast between the plume and the ground, which is an important factor for the MAP_SO $\mathrm{SO}_{2}$ algorithm to be able to detect and quantify $\mathrm{SO}_{2}$ emissions (Campion et al. 2010). This concept is examined further in the discussion (Section 5.4.) ASTER was able to view plumes in 3 images and quantify $\mathrm{SO}_{2}$ emissions from 1 image of Masaya on 9 February 2005, with a flux of $1362 \mathrm{t} \mathrm{d}^{-1}$. An average of $924 \mathrm{t} \mathrm{d}^{-1}$ of $\mathrm{SO}_{2}$ was emitted from Masaya. This average was calculated using the ASTER measurements along with data from Mather et al. (2006), Witt et al. (2008), and Nadeau et al. (2009).

ASTER imagery provided 5 images of $\mathrm{SO}_{2}$ plumes from San Cristobal volcano between 2002 and 2010. A daily average of $2141 \mathrm{t} \mathrm{d}^{-1}$ was calculated from the ASTER images and ground-based data (BGVN, 2000; Barrancos et al. 2008). The fluxes from the ASTER data ranged from 352-3444 $\mathrm{t} \mathrm{d}^{-1}$. NO $\mathrm{SO}_{2}$ was detected in ASTER images of Telica, Cerro Negro or Concepcion. Mather et al. (2006) obtained a flux of $530 \mathrm{t} \mathrm{d}^{-1}$ at Telica in November 2003, and the BGVN (2010) reported fluxes between 106-151 $\mathrm{t} \mathrm{d}^{-1}$ on 20 May 2009, giving an average flux of $354 \mathrm{t} \mathrm{d}^{-1}$ for Telica.

Concepcion has one $\mathrm{SO}_{2}$ flux measurement of $400 \mathrm{t} \mathrm{d}^{-1}$ from 19 August 2005 (BGVN, 2007). This measurement was obtained during an eruptive episode and so this flux is expected to be much higher than what Concepcion typically emits. Concepcion does have active fumaroles so there are definitely gas emissions, 
but how much is unknown. Without a more extensive data set, it is difficult to make assumptions about the activity and average emissions rate of $\mathrm{SO}_{2}$ at Concepcion.

\subsubsection{Costa Rica}

In Costa Rica, four volcanoes, Turrialba, Arenal, Poas and Irazu, were investigated and provided an $\mathrm{SO}_{2}$ emission rate of $0.7 \mathrm{Tg} / \mathrm{a}$. ASTER imaged 8 plumes at Turrialba, indicating an average flux of $2387 \mathrm{t} \mathrm{d}^{-1}$. In comparison to the average calculated by the OMI measurements, $1074 \mathrm{t} \mathrm{d}^{-1}$, the ASTER flux is much higher. Combining the two data sets together provides an average of $1638 \mathrm{t} \mathrm{d}^{-1}$ from 2007-2011.

An ASTER image of Arenal, on 29 August 2005, showed a weak plume with a maximum flux of $550 \mathrm{t} \mathrm{d}^{-1}$. Combined with the available ground-based data from Barrancos et al. (2008) an average flux of $215 \mathrm{t} \mathrm{d}^{-1}$ was calculated. Many cloudfree ASTER images were available of Poas. All the ASTER images mapped the crater area at Poas as $\mathrm{SO}_{2}$, with the crater lake having the highest column abundance, but no plume was evident. Due to this, no $\mathrm{SO}_{2}$ fluxes were derived from the ASTER imagery and published data were relied upon giving an average daily flux of $108 \mathrm{t} \mathrm{d}^{-1}$ (Mather et al. 2006; Barrancos et al. 2008). Such a low daily flux is below ASTER's detection limits therefore it is unable to detect any $\mathrm{SO}_{2}$ that has left the crater. 


\subsubsection{South American Volcanic Arc}

It was estimated that $3.7 \mathrm{Tg}$ of $\mathrm{SO}_{2}$ are emitted into the atmosphere every year from the South American volcanic arc. Table 5.4 displays the fluxes for each volcano, and the total for each country. This approximation was determined using a combination of OMI, ASTER, and data from published sources. ASTER only contributed to average fluxes at Lascar volcano, Chile, Ubinas, Peru, and Tungurahua, Ecuador. OMI and published data contributed heavily to produce an average flux for the South American arc especially in Ecuador and Colombia. Appendix 3 is a compilation of the data used to produce an average emission rate for the South American volcanic arc.

This estimate does not include emissions from eruptive events due to their sporadic nature and duration typically only lasting a few days (Andres and Kasgnoc 1998). An additional $0.4 \mathrm{Tg}$ are added to the estimate for large eruptions, such as Chaiten, Chile (2008) (Carn et al. 2008), Llaima, Chile (2008) (Global Sulfur Dioxide Monitoring Homepage) and Reventador, Ecuador (2002) (Carn et al. 2008). 
Table 5.4

$\mathrm{SO}_{2}$ fluxes for South American volcanoes (2000-2010) organized by country and a breakdown of averages calculated by each method.

\begin{tabular}{|c|c|c|}
\hline $\begin{array}{l}\text { Ecuador and } \\
\text { Colombia }\end{array}$ & \multicolumn{2}{|c|}{ Flux $\left(\mathrm{t} \mathrm{d}^{-1}\right)$ (baseline average) } \\
\hline Tungurahua & 825.0 & \\
\hline Reventador & 1211.5 & \\
\hline Galeras & 2220.0 & \\
\hline Nevado Del Huila & 1195.6 & \\
\hline \multirow{2}{*}{$\begin{array}{l}\text { Unknown plume } \\
\text { source } \mathrm{SO}_{2}(\mathrm{OMI})^{3 *}\end{array}$} & 1033.0 & \\
\hline & Total (Tg/a) & 2.5 \\
\hline \multicolumn{3}{|l|}{ Peru } \\
\hline Ubinas & 723.25 & \\
\hline \multirow[t]{2}{*}{ El Misti } & 674.5 & \\
\hline & Total & 0.3 \\
\hline \multicolumn{3}{|l|}{ Chile } \\
\hline Lascar & 882.6 & \\
\hline Lastarria & 233.0 & \\
\hline Planchon-Peteroa & 490.0 & \\
\hline Villarrica & 84.2 & \\
\hline \multirow[t]{2}{*}{ Llaima } & 630.0 & \\
\hline & Total & 0.9 \\
\hline Arc total & \multicolumn{2}{|c|}{$\begin{array}{l}9489+/-2846 \mathrm{t} \mathrm{d}^{-1}(12,067)^{4^{*}} \text { ol } \\
3.7+/-1.11 \mathrm{~T} \mathrm{~g} / \mathrm{a}(4.4)^{3^{*}}\end{array}$} \\
\hline
\end{tabular}

\footnotetext{
${ }^{3}$ :Represents emissions from OMI images where the source could not be pinpointed from a specific volcano.

4 "the total flux corrected for unmeasured $\mathrm{SO}_{2}$ emissions using the power law proposed by Brantley and Koepenick (1995).
} 


\subsubsection{Colombia and Ecuador}

OMI data were the primary source of information for the volcanoes in Ecuador and Colombia providing a regional flux of $2.5 \mathrm{Tg} / \mathrm{a}$. There were plenty of ground based data from published sources to supplement the OMI data, with an average flux of $3 \mathrm{~T}$ g/a. Nevado del Huila data were obtained from BGVN (2008) and the Instituto Colombiano de Geología y Minería (INGEOMINAS), and data for Galeras was from INGEOMINAS. Data for Tungurahua were from BGVN (2002), BGVN (2006) and Barrancos et al. (2008). The data sets were combined to give an average of $2.5 \mathrm{Tg} / \mathrm{a}$ for Colombia and Ecuador. It was not expected that any ASTER imagery would be useful for Ecuador and Colombia due to excessive cloud cover. This was reinforced by the fact that only one ASTER image was available for the region and quantified $\mathrm{SO}_{2}$ from Tungurahua (3 January 2001). An eruptive episode at Reventador in 2002 was not included in the average calculated for the volcano. The eruption was captured by TOMS and generated $0.3 \mathrm{Tg}$ (Carn et al. 2008).

\subsubsection{Peru and Chile}

An estimated volcanic $\mathrm{SO}_{2}$ flux of $0.3 \mathrm{Tg} / \mathrm{a}$ for Peru and $0.9 \mathrm{Tg} / \mathrm{a}$ for Chile was calculated. In Peru plumes were detected by ASTER and OMI at Ubinas. The 2 ASTER images of Ubinas that detected $\mathrm{SO}_{2}$ were taken during an eruptive episode that lasted from 2006-2009 (BGVN, 2006- 2010). Due to this eruptive episode spanning several years it is not considered a sporadic event and the $\mathrm{SO}_{2}$ fluxes are considered in calculating the baseline average for the volcano. OMI detected several plumes at Ubinas. Figure 5.7 illustrates some of the plumes seen from Ubinas

ASTER imagery was processed for Lascar, Lastarria, Irruputuncu, Villarrica, Llaima, and Planchon-Peteroa in Chile, and Copahue, Argentina. ASTER successfully detected $\mathrm{SO}_{2}$ plumes in 15 images from Lascar and one from Planchon-Peteroa. OMI was able to detect plumes at Lascar (refer to Chapter 4 
for more detail) Lastarria and Villarrica. An average $\mathrm{SO}_{2}$ emission rate of $882 \mathrm{t}$ $\mathrm{d}^{-1}$ was calculated for Lascar. It was expected that emissions would be detected from Villarrica and Llaima using ASTER, due to ground based measurements indicating fluxes from $100 \mathrm{t} \mathrm{d}^{-1}$ to over a $1000 \mathrm{t} \mathrm{d}^{-1}$ for Villarrica (Witter et al. 2004; Mather et al. 2004; Palma et al. 2008) and $600 \mathrm{t} \mathrm{d}^{-1}$ for Llaima (Mather et al. 2004). OMI was able to detect emissions at Villarrica and an average of $62 \mathrm{t}$ $\mathrm{d}^{-1}$ was calculated from $2004-2010$. The OMI flux is small compared to the flux calculated from published data (COSPEC and DOAS). Combining the data produced an average flux of $84 \mathrm{t} \mathrm{d}^{-1}$ for Villarrica.

Emission rates from Irruputuncu are unknown, but reports of a visible plume above the crater (Tassi et al. 2011) indicated that perhaps it was a good target for emissions to be quantified by ASTER and OMI. Neither instrument detected $\mathrm{SO}_{2}$ at Irruputuncu. 

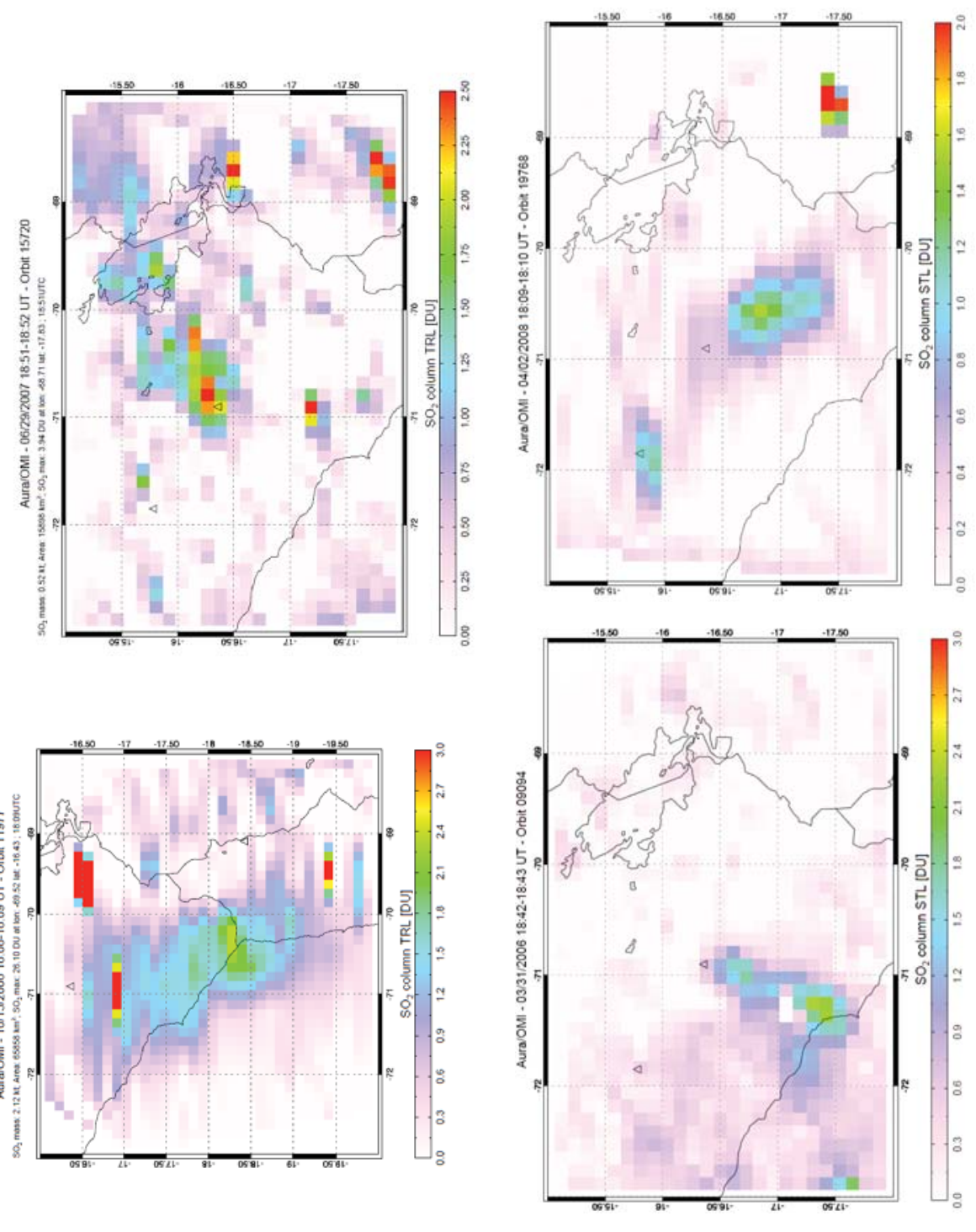

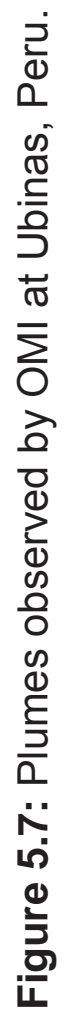




\subsection{Discussion}

\subsubsection{Comparison to Previous Estimates}

This study has estimated that emissions from the Central American and South American arc contributed $3.4 \pm 1$ and $3.7 \pm 1.1 \mathrm{Tg} / \mathrm{a}$, respectively, to the atmosphere from 2000-2010. Previous estimates have included country specific estimations made by Andres et al. (1993) for Guatemala and Rodriguez et al. (2004), for Guatemala and El Salvador. Their estimates were $0.18 \mathrm{~T}$ g/a and 0.28-1.45 Tg/a, respectively. For Guatemala we reported an average of 0.7 $\mathrm{Tg} / \mathrm{a}$ and $0.8 \mathrm{Tg} / \mathrm{a}$ for El Salvador to produce a combined estimate of $1.5 \mathrm{Tg} / \mathrm{a} \pm$ 0.4 Our result is much higher than that produced by Andres et al. (1993) but at the maximum reported by Rodriguez et al. (2004), for Guatemala and El Salvador.

Arc wide estimations have been made by Halmer et al. (2002) and Mather et al. (2006) with values of 0.6-0.8 Tg/a and 1.6 Tg/a \pm 0.5 , respectively, for the Central American arc and 2.1-3.6 Tg/a by Halmer et al. (2002) for the South American arc. Our approximation for Central America, $3.4 \pm 1 \mathrm{Tg} / \mathrm{a}$, is much greater than the estimates produced by Halmer et al. (2002) and Mather et al. (2006). The estimate for the South American arc, $3.9 \pm 1.2 \mathrm{Tg} / \mathrm{a}$, is larger than that estimated by Halmer et al. (2002), but lies within the error margins.

Both estimates are higher than previous fluxes made for the volcanic arcs. This is due to several reasons. Firstly, previous studies have predominantly used ground-based measurements from volcanoes. Ground based measurements are not always taken continuously. For example the measurements by Rodriguez et al. (2004) and Mather et al. (2006) were taken during short field campaigns and may not reflect long term degassing trends. ASTER did not provide a continuous data set for any of the volcanoes such as Pacaya and Fuego, Guatemala. OMI data did provide extensive datasets for several 
volcanoes such as Villarrica, Chile and Turrialba, Costa Rica however, the data was not continuous.

The satellite data incorporated into the study by Halmer et al. (2002) were from TOMS. TOMS had a detection limit of 5- $10 \mathrm{kt}\left(\sim 7000 \mathrm{t} \mathrm{d}^{-1}\right)$ (Krotkov et al. 2006; Carn et al. 2007; 2008) and was only useful for detecting large quantities of $\mathrm{SO}_{2}$ from volcanoes typically in an eruptive state. The majority of the volcanoes in Central and South America are passively degassing, emitting $\mathrm{SO}_{2}$ into the lower troposphere, with fluxes typically less than $\sim 1000 \mathrm{t} \mathrm{d}^{-1}$, which means they cannot be detected by TOMS. This study has demonstrated that ASTER and $\mathrm{OMI}$, with their lower detection limits, can detect emissions from many of the volcanoes in Central and South America.

Without the use of OMI many of the volcanoes in South America would not have an extensive $\mathrm{SO}_{2}$ data set, especially the volcanoes in Ecuador and Colombia. ASTER was only able to detect one plume in this region, from Tungurahua. Extensive cloud cover in this region made it difficult for ASTER to determine if any $\mathrm{SO}_{2}$ plumes were present. OMI, operating in the $\mathrm{UV}$, is able to detect $\mathrm{SO}_{2}$ even under cloudy conditions. However, it should be noted that meteorological clouds can shield $\mathrm{SO}_{2}$ in the atmosphere and artificially enhance the signal of $\mathrm{SO}_{2}$ if the plume is above clouds (Krotkov et al. 2006). A yearly flux of $2.8 \mathrm{Tg} / \mathrm{a}$ was estimated from volcanoes in Ecuador and Colombia. Measurements primarily came from Tungurahua, Reventador, Galeras, and Nevado del Huila. An $\mathrm{SO}_{2}$ mass of $1.16 \mathrm{Tg}$ was calculated for this region over a 2-year period (from September 2004 - September 2006) by Carn et al. (2008). Our yearly average is more than double this value $(2.8 \mathrm{Tg} / \mathrm{a})$. The two estimates cannot be fairly compared due to there being too much inter-annual variability. This enforces the importance of using a longer time scale to remove this variability. 


\subsubsection{OMI}

$\mathrm{SO}_{2}$ estimated from $\mathrm{OMI}$ data are reported in mass (kilotons) for the entire plume, not as a flux in $\mathrm{t} \mathrm{d}^{-1}$. A SO $\mathrm{SO}_{2}$ flux can be calculated by taking a single transect through the plume close to the vent and using the plume speed (wind speed). With the exception of volcanoes in Colombia and Ecuador, daily fluxes were calculated using a transect. Due to time constrains, the mass of the plumes produced from Colombian and Ecuadorian volcanoes were crudely converted to a flux. The $\mathrm{SO}_{2}$ mass in kilotons was converted to tons by multiplying the mass by 1000 . Using the mass of the entire plume will add error to the estimate and has some problems associated with it due to mass and flux not being the same quantity.

An OMI image of the entire plume may range from less than a day, to several days worth of $\mathrm{SO}_{2}$ emissions from a volcano. An OMl image is taken of a volcano once a day at low latitudes. If the $\mathrm{SO}_{2}$ emissions and $\mathrm{SO}_{2}$ lifetime are large enough and constantly producing an extensive plume, the $\mathrm{SO}_{2}$ may not be removed from the atmosphere by the time the next $\mathrm{OMI}$ image is acquired. Thus, the image may contain several days worth of $\mathrm{SO}_{2}$ from a volcano. Care must be taken when looking at consecutive days of OMI imagery at a volcano and the speed of tropospheric $\mathrm{SO}_{2}$ loss must be considered. For Turrialba, Fuego/Pacaya, and Villarrica this was not an issue. Many of the OMI images were not on consecutive days. In Ecuador and Colombia, where OMI was heavily relied upon, for certain volcanoes such as Nevado del Huila, there was a large quantity of OMI data with many measurements over successive days.

It is expected that using the mass of the plume will provide an overestimation of $\mathrm{SO}_{2}$, especially where an average is determined using a data set that contains many images collected over consecutive days. It would be expected that the average emission rates produced for OMI would be higher than what was estimated from other sources of data such as ASTER and published data. This 
is not the case. Table 5.5 provides a breakdown of the average emission rates for some of the volcanoes in this study. Averages calculated by OMI are compared to averages calculated from other sources of data used in this study. It is seen that all the average emission rates calculated for volcanoes from OMI data were lower than those obtained from ASTER and published ground based data combined. Studies by Bani et al. (2012) and McCorimick et al. (2012) illustrated that OMI measurements were much lower than corresponding DOAS measurements in the Vanuatu island arc and from volcanoes in Papua New Guinea. These studies suggest that OMI produces lower estimates of total $\mathrm{SO}_{2}$ emissions (McCormick et al. 2012). Lower $\mathrm{OMI} \mathrm{SO}_{2}$ burdens are thought to be a direct result of the altitude assumption in the $\mathrm{OMI} \mathrm{SO}_{2}$ retrieval, meteorological clouds and rapid $\mathrm{SO}_{2}$ loss rates in a tropical region (McCormick et al. 2012; Bani et al. 2012). McCormick et al. 2012 suggests that the primary reason for lower OMl estimates is due to the dispersion and chemical processing of the plume.

Table 5.5

Average daily $\mathrm{SO}_{2}$ emissions from OMI compared to other sources of data at volcanoes where $\mathrm{OMI}$ detected $\mathrm{SO}_{2}$ plumes ${ }^{5}$.

\begin{tabular}{|c|c|c|}
\hline Volcano & $\begin{array}{c}\text { OMI Average Daily } \\
\text { Emissions }\end{array}$ & $\begin{array}{c}\text { Other sources of } \mathrm{SO}_{2} \text { Flux } \\
\text { data }\left(\mathrm{t} \mathrm{d}^{-1}\right) \\
\text { (ASTER and ground based } \\
\text { data from published sources) }\end{array}$ \\
\hline Nevado del Huila & 1444 & 4260 \\
\hline Tungurahua & 750 & 1253 \\
\hline Galeras & 572 & 2336 \\
\hline Turrialba & 1638 & 2387 \\
\hline Villarrica & 62 & 389 \\
\hline
\end{tabular}

\footnotetext{
${ }^{5}$ OMI emission rate calculated using a transect taken across the plume for Turrialba. The other emissions rates are fluxes calculated from the mass of $\mathrm{SO}_{2}$ in a plume.
} 
With the potential problem of multiple days' worth of $\mathrm{SO}_{2}$ emissions in a single $\mathrm{OMI}$ image, the speed of $\mathrm{SO}_{2}$ loss in to the troposphere is very important. Loss rate is dependent on many factors such as humidity, precipitation, solar radiation, cloud cover, temperature, and the local meteorology (Oppenheimer et al. 1998; Horrocks et al. 2003; Rodriguez et al. 2008). In a dry atmosphere 1$10 \%$ loss every hour is expected (Eatough et al. 1994; McGonigle et al. 2004). McGonigle et al. (2004) calculated loss rates at Masaya where there is a relatively slow loss rate of $10^{-5} \mathrm{~s}^{-1}$ (i.e., an average $\mathrm{SO}_{2}$ lifetime of 28 hours).

In humid environments, loss is faster and estimates have been made by Oppenheimer et al. (1998) and Rodriguez et al. (2008) at Soufriere Hills Volcano, Montserrat. Oppenheimer et al. (1998) deduced a fast $\mathrm{SO}_{2}$ loss rate of $10^{-3} \mathrm{~s}^{-1}$ (i.e., an average $\mathrm{SO}_{2}$ lifetime of $\sim 17$ minutes). Rodriguez et al. (2008) calculated $\mathrm{SO}_{2}$ loss rates with an average of $10^{-4} \mathrm{~s}^{-1}$ which equates to an $\mathrm{SO}_{2}$ lifetime of $\sim 2.78$ hours. The plumes quantified by OMI are typically in a tropical atmosphere and are expected to have dissipated within 24 hours. Arellano et al. (2008) estimated that the average lifetime of a passive plume at Tungurahua is $\sim 1$ day. Thus the problem of OMI images containing several days' worth of $\mathrm{SO}_{2}$ emissions from passively degassing emissions is not an issue.

\subsubsection{Errors}

There are a number of sources of error in this study. The initial errors are related to the ASTER and OMI instruments and the algorithms used to quantify $\mathrm{SO}_{2}$. The errors for each instrument are described in detail in Chapter 1 and only briefly mentioned here. Errors related to ASTER include instrument calibration and sensitivity, the accuracy of the mapping procedure and how the radiation from the ground to the sensor is described, plume geometry, and accuracy of the atmospheric data. OMI errors typically increase with larger quantities of $\mathrm{O}_{3}$ and $\mathrm{SO}_{2}$, the presence of clouds and aerosols, and solar zenith angle (Krotkov et al. 2006). Ash and haze also have an impact on the retrieval 
of $\mathrm{SO}_{2}$ from OMI data (Krotkov et al. 2006). The maximum errors for ASTER are $\sim 31 \%$ (Henney 2006) and OMI are $\sim 30-70 \%$ (Carn et al. 2008). An error range of $\pm 30 \%$ is added to the total arc wide fluxes produced here to account for instrumental errors (see Tables 5.3 and 5.4).

There are errors associated with the data collection and estimation of $\mathrm{SO}_{2}$ for the 2 arcs. The way the volcanoes are sampled differs depending on the volcano. Some volcanoes have data collected at them more frequently than others. Masaya for example has been extensively monitored and over $70 \mathrm{SO}_{2}$ fluxes, over a 10-year period, were used to calculate a baseline average in this study. These 70 measurements are comprised of ASTER and ground based measurements obtained by Mather et al. (2006), Witt et al. (2008) and Nadeau et al. (2009). At the other end of the scale are volcanoes such as Tacana or Llaima, where only one measurement is representative of the average quantity of $\mathrm{SO}_{2}$ emitted into the troposphere every year. Baseline averages are important in terms of monitoring volcanoes and potentially predicting impending eruptions. An extensive data set of gas measurements can be used to look for patterns of activity that relate to eruptive behavior. When few measurements are available for a volcano, such as Tacana or Llaima, it is hard to comment on or describe the characteristics of that volcano and its activity. The averages assumed from these volcanoes where there is a single measurement are misleading and inaccurate. To account for gaps in the data, it has been suggested that the data be interpolated (Halmer et al. 2002; Rodriguez et al. 2004). Again, where few measurements are available at a volcano this task is difficult. To resolve this issue an increase in the temporal resolution of measurements is needed.

Not all the volcanoes within the two volcanic arcs were sampled in this study. Such examples of volcanoes where data was acquired but no $\mathrm{SO}_{2}$ was detected include, but are not limited to Irruputuncu (Chile) and Cerro Negro 
(Nicaragua). To correct for unmeasured $\mathrm{SO}_{2}$ fluxes a method described by Brantley \& Koepenick (1995) is employed. The method is based on an assumed power law of the distribution of volcanic fluxes on arc wide and global scales:

$$
\mathrm{N}=\mathrm{af}^{-\mathrm{c}}
$$

where $\mathrm{N}$ is the number of volcanoes with an $\mathrm{SO}_{2}$ emission rate $\geq f$ and $a$ and $\mathrm{c}$ are constants. If the constant $\mathrm{c}$ is $<1$ then the following equation from Marrett and Almendinger (1991) can be applied to the data to calculate the total regional flux $\left(f_{\text {tot }}\right)$ :

$f_{\text {tot }}=f_{1}+f_{2}+f_{3}+\ldots+f_{N}\left[c /(1-c)(n+1)(n /(n+1))^{\wedge}(1 / c)\right]$

where, $f_{N}$ is the Nth largest flux. A c value of 0.51 and 0.28 were calculated for the Central and South American arcs respectively (Figure 5.8).

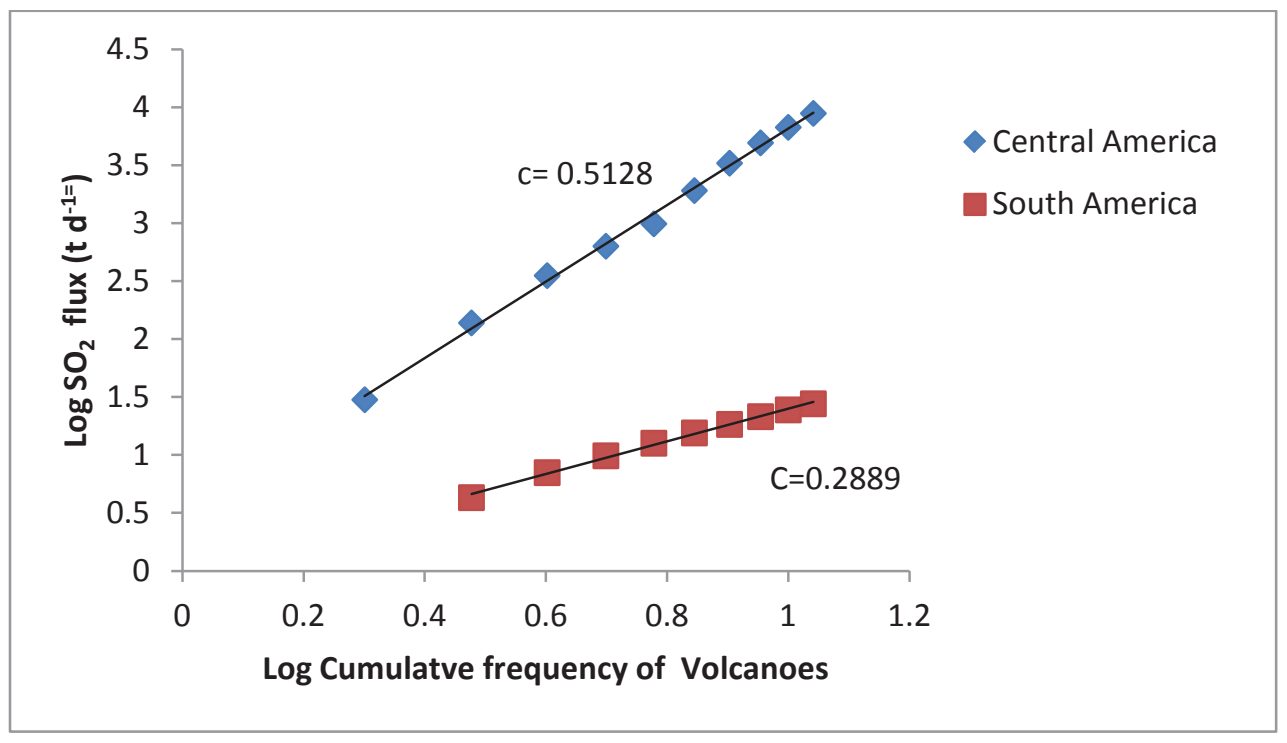

Figure 5.8: Plot of Log of volcano frequency versus $\mathrm{Log}$ of $\mathrm{SO}_{2}$ emissions illustrating the value of constant $\mathrm{c}$. 
Fluxes of $1919 \mathrm{t} \mathrm{d}^{-1}$ and $1904 \mathrm{t} \mathrm{d}^{-1}$ were estimated for volcanoes where no measurements were obtained in Central and South America respectively. The unmeasured fluxes account for $17 \%$ for the Central American arcs and $15 \%$ South American arcs overall flux. The power law is expected to overestimate smaller emitters of $\mathrm{SO}_{2}$ (Andres \& Kasgnoc 1998) thus the values calculated here are maximum estimates of unmeasured $\mathrm{SO}_{2}$.

\subsubsection{Detection Limits}

This study has provided an opportunity to determine how effective ASTER is at detecting $\mathrm{SO}_{2}$ under varying atmospheric conditions, different altitudes, ground conditions, and types of volcanoes. Previous studies have indicated that the algorithm has a limited dependence on the atmospheric humidity, surface altitude, and emissivity of the ground (Henney 2006; Campion et al. 2010). Work carried out by Realmuto (2000) using simulated data, indicated that ASTER would have no problem detecting emissions from Kilauea and Etna. Henney (2006) used a forward model to simulate ASTER data of plumes from Fuego, Guatemala. It was determined that ASTER is capable of detecting emissions as low as $500 \mathrm{t} \mathrm{d}^{-1}$ from Fuego (a $4 \mathrm{~km}$ high plume in a tropical atmosphere). Table 5.6 outlines the altitude and average $\mathrm{SO}_{2}$ flux emitted from specific volcanoes in the two arcs. The table also indicates if ASTER detected any $\mathrm{SO}_{2}$ at the volcano and if yes, the lowest quantity ASTER detected. 
Table 5.6

Volcanoes studied and average $\mathrm{SO}_{2}$ emissions detected from them using ASTER.

\begin{tabular}{|c|c|c|c|c|c|}
\hline Volcano & $\begin{array}{c}\text { Vent } \\
\text { Altitude } \\
\quad(\mathrm{m})\end{array}$ & $\begin{array}{c}\text { Average } \\
\mathrm{SO}_{2} \text { flux } \\
\left(\mathrm{t} \mathrm{d}^{-1}\right)\end{array}$ & $\begin{array}{c}\text { Can } \\
\text { ASTER } \\
\text { detect } \\
\mathrm{SO}_{2}\end{array}$ & $\begin{array}{l}\text { Lowest } \mathrm{SO}_{2} \\
\text { flux } \\
\text { detected by } \\
\text { ASTER ( } \mathrm{d}^{-} \\
\left.{ }^{1}\right)\end{array}$ & $\begin{array}{c}\text { Number of } \\
\text { ASTER } \\
\text { images } \\
\text { that } \\
\text { detected } \\
\mathrm{SO}_{2}\end{array}$ \\
\hline Pacaya & 2252 & 1382 & Yes & 233 & 5 \\
\hline Fuego & 3763 & 354 & Yes & 532 & 1 \\
\hline Santiaguito & 2500 & 123 & No & - & \\
\hline Santa Ana & 2351 & 1783 & No & - & \\
\hline San Miguel & 2130 & 215 & No & - & \\
\hline Telica & 1061 & 354 & No & - & \\
\hline $\begin{array}{l}\text { San } \\
\text { Cristobal }\end{array}$ & 1745 & 2141 & Yes & 352 & 6 \\
\hline Masaya & 635 & 924 & Yes & 1362 & 1 \\
\hline Poas & 2708 & 108 & No & - & \\
\hline Arenal & 1670 & 215 & Yes & 550 & 2 \\
\hline Turrialba & 3340 & 902 & Yes & 219 & 7 \\
\hline Ubinas & 5672 & 723 & Yes & 2397 & 2 \\
\hline Lascar & 5592 & 882 & Yes & 122 & 15 \\
\hline Lastarria & 5697 & 233 & No & & \\
\hline Irruputuncu & 5163 & - & No & - & \\
\hline Llaima & 3125 & 630 & No & - & \\
\hline Villarrica & 2847 & 84 & No & - & \\
\hline
\end{tabular}


No $\mathrm{SO}_{2}$ was detected by ASTER at several of the volcanoes located high in the Andes, where it is expected that $\mathrm{SO}_{2}$ would be easily detected due to the relatively dry climate and high altitude of the volcanoes and their plumes. Villarrica, Llaima, and Irruputuncu are known emitters of $\mathrm{SO}_{2}$ (Mather et al. 2004; Witter et al. 2004; Palma et al. 2008; Sawyer et al. 2011; Tassi et al. 2011). Over 30 ground-based measurements have been taken at Villarrica and fluxes have ranged from $80 \mathrm{t} \mathrm{d}^{-1}$ to $1300 \mathrm{t} \mathrm{d}^{-1}$ (Witter et al. 2004; Mather et al. 2004; Palma et al. 2008; Sawyer et al. 2011). An average of $84 \mathrm{t} \mathrm{d}^{-1}$ was calculated here. Field campaigns by Mather et al. (2004) and Sawyer et al. (2011) determined average $\mathrm{SO}_{2}$ fluxes of $\sim 350 \mathrm{t} \mathrm{d}^{-1}$. Only one measurement has been taken at Llaima by Mather et al. (2004) and a flux of $630 \mathrm{t} \mathrm{d}^{-1}$ was determined. This flux was during a period of elevated activity and is not likely to be representative of Llaima's average flux. OMI did detect a large plume from Llaima in January 2008, with a mass of $30 \mathrm{kt}$, and in April 2009 (Global Sulfur Dioxide Monitoring Homepage, http://so2.gsfc.nasa.gov/). No data have been collected for Irruputuncu, but it has been reported that there is a plume seen above the volcano (Tassi et al. 2011).

Not a single ASTER image was able to detect $\mathrm{SO}_{2}$ at Villarrica, Llaima, and Irruputuncu. This was unexpected given that other volcanoes of lower or similar altitude had plumes detected from them. It is possible that the $\mathrm{SO}_{2}$ fluxes were too low on the days that ASTER imagery was acquired. Lascar volcano produced emissions that were intermittently detected by ASTER. Looking at the data more closely it was noted that the majority of the ASTER images that revealed plumes at Lascar were daytime images. Nighttime images rarely observed a plume. The pixels around the crater at Lascar were found to be significantly colder in the nighttime images when compared to daytime images. Adequate thermal contrast between the plume and the ground is critical for $\mathrm{SO}_{2}$ retrievals in the TIR (Realmuto 1994; 1997; Campion et al. 2010). Typically the $\mathrm{SO}_{2}$ plume is cooler than the ground. When the ground and the plume are the 
same temperature the thermal contrast is lost and no $\mathrm{SO}_{2}$ can be detected by the MAP_SO2 algorithm. The sensitivity of the retrieval increases as the thermal contrast increases (Campion et al. 2010). Since no plumes can be detected at Villarrica, Llaima, and Irruputuncu it is assumed that there is either no thermal contrast between the plume and the ground at these volcanoes or the $\mathrm{SO}_{2}$ emissions were too low to detect. All three of these volcanoes are covered in ice or snow making it a distinct possibility that the ground temperature is as cold as the plumes they are emitting.

Typically higher plumes are easier to detect than lower plumes. Of course this statement is not completely true and has one caveat where thermal contrast is an issue as seen by plumes emitted from Villarrica, Llaima, Irruputuncu, and Lascar to a certain extent. Plumes from Lascar have been detected with emissions as low as $\sim 150 \mathrm{t} \mathrm{d}^{-1}$. On the other end of the scale is Masaya. Masaya is the lowest lying volcano within the 2 arcs and the plume there often flows down the flanks of the volcano hugging the ground. An ASTER image from 2-9-2005 was able to detect a plume with a $\mathrm{SO}_{2}$ emission rate of $1362 \mathrm{t} \mathrm{d}^{-}$ 1 . There were many other ASTER images available of Masaya where it was evident there was $\mathrm{SO}_{2}$ present. Many of these images showed $\mathrm{SO}_{2}$ confined to the crater. The average $\mathrm{SO}_{2}$ emitted from Masaya is close to $1000 \mathrm{t} \mathrm{d}^{-1}$ with fluxes ranging from $105 \mathrm{t} \mathrm{d}^{-1}$ to $3224 \mathrm{t} \mathrm{d}^{-1}$. This average is calculated from ASTER and ground based measurements. For emissions to be detected by ASTER at such a low altitude in a tropical environment it is clear that a flux greater than $1000 \mathrm{t} \mathrm{d}^{-1}$ is required. 


\subsection{Conclusions}

An $\mathrm{SO}_{2}$ flux of $3.4 \mathrm{Tg} / \mathrm{a}$ for the Central American volcanic arc and $3.7 \mathrm{Tg} / \mathrm{a}$ for the South American volcanic arc were estimated using a combination of ASTER, OMl and ground based data from published sources.

These estimations are higher than those previously calculated for a variety of reasons. ASTER and OMI have lower $\mathrm{SO}_{2}$ detection limits than other sensors used in previous work such as TOMS. This has allowed emissions from low emitters of $\mathrm{SO}_{2}$ to be detected. ASTER and OMI also aided in taking routine measurements at several of the volcanoes over an extensive period of time, not just during eruptive episodes or field campaigns. However, estimates may be higher than those previously estimated due to some volcanoes having a higher average emissions rate than expected. Such an example is Pacaya, Guatemala, where the average daily emission rate $\left(1382 \mathrm{t} \mathrm{d}^{-1}\right)$ calculated from our limited data set is higher than previous averages estimated. Additionally, it would be expected that ASTER would capture more plumes at Pacaya if $1382 \mathrm{t}$ $\mathrm{d}^{-1}$ was being emitted during the study period. Another example is Concepcion, Nicaragua, where one data point is available for the volcano and is used to represent the average flux for the volcano.

When averages calculated from OMI data were compared to ASTER and ground based data, it was found they were lower. This may be due to the fact that the data set was more continuous with more data points. Emissions detected by ASTER were often high. For example, the plumes detected at Pacaya were all $>1000 \mathrm{t} \mathrm{d}^{-1}$. With ASTER only detecting high emission rates, not being able to detect smaller plumes (lower emissions), and not providing continuous data the average flux calculated would be much higher than it really is. This is problematic when calculating baseline fluxes. 
Many volcanoes only had 1-2 measurements taken at them and some volcanoes (e.g., Telica and Tacana) emit too little $\mathrm{SO}_{2}$ to be detected by current satellite-based remote sensors. Continuous ground based measurements need to be conducted at these volcanoes to improve on the estimates made here (Galle et al. 2010; Vita et al. 2012). Additionally, it is important to use a variety of techniques to determine an average flux as no single instrument is capable of measuring $\mathrm{SO}_{2}$ at all volcanoes due to temporal and spatial resolution, location, and cost. Many studies exist where direct samples have been taken of the gasses at volcanoes. A next step in this project, to make a more complete record of $\mathrm{SO}_{2}$ emissions, would to be include data obtained from direct sampling. Such an example is the work carried out by Witt et al. (2008) where direct sampling studies were carried out at Masaya, Telica, San Cristobal, and Momotombo volcanoes, Nicaragua.

The detection limits of ASTER were explored and indicated some problems that the sensor and MAP_SO2 algorithm have when quantifying $\mathrm{SO}_{2}$. In general, higher plumes were easier to detect than lower plumes. ASTER is capable of detecting as little as $\sim 150 \mathrm{t} \mathrm{d}^{-1}$ for high altitude volcanoes (plumes) $(>5 \mathrm{~km}$ ) under ideal conditions, such as a dry climate. Lower plumes, such as that from Masaya $(<1 \mathrm{~km})$, need higher column abundances of $\mathrm{SO}_{2}$ to be detected. At Masaya the $\mathrm{SO}_{2}$ flux needs to be in excess of $\sim 1000 \mathrm{t} \mathrm{d}^{-1}$ to be detected by ASTER. These detection limits are a general guide and cannot be applied to all plumes such as those from Villarrica, Llaima, and Irruputuncu where the thermal contrast between the ground and the plume was problematic. Where there is no thermal contrast the MAP_SO2 algorithm cannot effectively detect and quantify $\mathrm{SO}_{2}$. The ground at these volcanoes was cold due to high elevation and snow or ice cover. This resulted in the plume being apparently the same temperature as the ground meaning there was no thermal contrast between the two. 
Further work concerning the contrast between the plume and the ground needs to take place. No $\mathrm{SO}_{2}$ was found in any of the nighttime images of Lascar from 2000-2011, which is odd considering fluxes were often in excess of $500 \mathrm{t} \mathrm{d}^{-1}$ (refer to Chapter 4 for details). The ground temperature in all of these images was $\sim-4^{\circ} \mathrm{C}$. It is assumed that there is a plume in many of these images and it is as cold as the ground. Ground and plume temperatures need to be analyzed at all the volcanoes in this study where the temperature contrast is thought to be an issue. Also, to determine if a plume might be present image enhancement techniques should also be employed. 


\section{Chapter 6}

\section{Summary and Conclusions}




\section{Summary and Conclusions}

The Advanced Spaceborne Thermal Emission and Reflection Radiometer (ASTER) has proven to be a valuable tool for quantifying volcanic $\mathrm{SO}_{2}$. Through extensive data processing of $\mathrm{SO}_{2}$ plumes from a variety of volcanoes and a satellite validation, some important conclusions can be made about ASTER and it capabilities. The plume altitude is critical when processing ASTER data for $\mathrm{SO}_{2}$. The plume altitude is important in terms of the thermal contrast. ASTER can detect plumes at higher altitudes more easily than those at lower altitudes. Thus, as the plume altitude becomes lower the $\mathrm{SO}_{2}$ column abundance must increase for ASTER to be capable of detecting it. At Lascar volcano, Chile, (altitude of $6 \mathrm{~km}$ ) the plume is above $\sim 6.5 \mathrm{~km}$ altitude and ASTER was capable of detecting as little as $\sim 150 \mathrm{t} \mathrm{d}^{-1}$. At the opposite end of the spectrum is Masaya where the plume is low, below $1 \mathrm{~km}$, and often hugs the ground. Emissions must be in excess of $\sim 1300 \mathrm{t} \mathrm{d}^{-1}$ to be detected by ASTER. There is a caveat to these general recommendations. Where there is no temperature contrast between the plume and the ground $\mathrm{SO}_{2}$ cannot be detected. ASTER could not detect plumes from many volcanoes in the Central Andes due to a lack of temperatures contrast between the plume and ground. Campion et al. (2010) have investigated this problem using computer simulations. Further work needs to be carried out to explore this problem. A scheduled ASTER overpass of one of the volcanoes where temperature contrast is a problem might be of benefit. If visual observations and ground-based measurements can be obtained at the same time, then the issue of temperature contrast can be explored further. Plume temperature and $\mathrm{SO}_{2}$ flux could be quantified to prove that temperature contrast is an issue at the volcanoes in this study.

Satellite validation is important to ensure that the general guidelines made above are valid along with the fluxes produced for the ASTER images analyzed in this research. ASTER data were compared to ground data collected by a Mini UV Spectrometer (MUSe) at Lascar volcano, Chile. ASTER were found to be a 
reliable detector of $\mathrm{SO}_{2}$ and advantageous for producing a $2 \mathrm{D}$ map of the whole plume, not just a transect like the MUSe. This allowed the plume to be analyzed through the use of a timeline, increasing the temporal resolution of the ASTER data. Further steps in the validation of ASTER should be to compare the sensor to other satellite based sensors such as the Ozone Monitoring Instrument (OMI). ASTER is capable of producing comparable results to local ground based measurements but how will it compare to instruments with larger spatial resolutions.

One of the major problems with the ASTER data was that the plume was not always visually discernible in the raw thermal infrared (TIR) data. With the quantity of ASTER data assessed it was important to be able to disregard the images where no plumes were identified before they were processed. This was done using the Principal Components Analysis and Decorrelation Stretch image enhancing techniques. These techniques are transferable to other data such as MODIS. These enhancements were quick and simple, reducing the time spent on processing images where no plume exists.

With ASTER's detection limits and temporal resolution, a continuous dataset is not possible at volcanoes throughout Central and South America. ASTER was most successful at detecting plumes at Lascar, Chile, where $\mathrm{SO}_{2}$ was detected in 26 images. Fluxes could be quantified from 16 of the images to produce a $\mathrm{SO}_{2}$ timeline between 2000 and 2011. Using this data alone, no assumptions could be made about the activity at the volcano. However, when visual observations and temperature data were combined with the $\mathrm{SO}_{2}$ timeline it could be deduced that Lascar is at the end of an eruptive cycle that started in 1993. With the $\mathrm{SO}_{2}$ emissions slowly decreasing over time it is concluded that the conduit is sealing.

The emission rates from the volcanoes in the Central and South America volcanic arcs between 2000-2010 were determined with fluxes of $3.4 \mathrm{Tg} / \mathrm{a}$ and 
3.7 Tg/a of $\mathrm{SO}_{2}$ were emitted respectively. Without a combination of ASTER, $\mathrm{OMI}$ and published data the averages calculated here would not have been possible. Improvements on the averages can be made with the use of additional data from direct sampling studies or employing other sensors such as the Moderate Resolution Imaging Spectroradiometer (MODIS). With the OMI data being expressed as a mass a further step would be to provide daily fluxes for all these images to improve on the estimates produced in this work.

Where ASTER data were not continuous and emissions are below the detection limits of the sensor, baseline averages calculated for a volcano were often too high, e.g. Pacaya, Guatemala and Turrialba, Costa Rica. With the use of $\mathrm{OMI}$ a more continuous dataset was produced. OMI produces daily images providing more opportunities to detect $\mathrm{SO}_{2}$ plumes making the data set more complete at volcanoes such as Turrialba. It is also important to use multiple sources of data when viewing volcanic plumes to obtain multiple data points and a more complete dataset to calculate more accurate baseline fluxes. 


\section{Reference List}

Abrams M (2000) ASTER: data products for the high spatial resolution imager on NASA's EOS-AM1 platform. International Journal of Remote Sensing, 21: 847-861.

Allard P, Carbonelle J, Métrich N, Loyer H, Zettwoog P (1994) Sulphur output and magma degassing budget of Stromboli volcano. Nature, 368: 326330.

Allard P (1997) Endogenous magma degassing and storage at Mount Etna. Geophysical Research Letters, 24: 2219-2222.

Allen AG, Baxter PJ, Ottley CJ (2000) Gas and particle emissions from Soufriere Hills Volcano, Montserrat, West Indies: characterization and health hazard assessment. Bulletin of Volcanology 62: 8-19.

Alley RE (1996) Algorithm Basis Document for Decorrelation Stretch. Jet Propulsion Laboratory, Pasadena, California.

Andres RJ, Rose WI, Kyle PR, Silva S de, Francis P, Gardeweg M, Moreno RH (1991) Excessive sulfur dioxide emissions from Chilean Volcanoes. Journal of Geothermal Research 46:323-329.

Andres R J, Rose W I, Stoiber RE, Williams SN, Matias O, Morales R (1993). A summary of sulfur dioxide emission rate measurements from Guatemalan volcanoes Bulletin of Volcanology 55(5): 379-388. 
Andres RJ, Kasgnoc AD (1998) A time-averaged inventory of subaerial volcanic sulfur emissions. Journal of Geophysical Research 103 (D19): 2525125261.

Arellano SR, Hall M, Samaniego P, Le Pennec KL, Ruiz A, Molina I, Yepes H (2008) Degassing patterns of Tungurahua volcano (Ecuador) during the 1999-2006 eruptive period, inferred from remote spectroscopic measurements of $\mathrm{SO}_{2}$ emissions. Journal of Volcanology and Geothermal Research, 176: 151-162.

Bani P, Oppenheimer C, Allard P, Shinohara H, Tsanev V, Carn S, Lardy M, Garaebiti E (2012) First estimate of volcanic $\mathrm{SO}_{2}$ budget for Vanuatu island arc. Journal of Volcanology and Geothermal Research, 211212:36-46.

Barckhausen U, Ranero CR, von Huene R, Cande SC, Roeser HA (2001) Revised tectonic boundaries in the Cocos plate off Costa Rica: Implications for the segmentation of the convergent margin and for plate tectonic models. Journal of Geophysical Research, 106: 19207-19220.

Barrancos J, Rosello J, Calvo D, Padron E, Melian G, Hernandez PA, Perez NM, Millan M, Galle B (2008) $\mathrm{SO}_{2}$ emissions from active volcanoes measured simultaneously by COSPEC and minl-DOAS. Pure and applied geophysiscs 165(1): 115-133.

Bartels OG (1972) An Estimate of Volcanic Contributions to the Atmosphere and Volcanic Gases and Sublimates as the Source of the Radioisotopes 10Be, 35S, 32P and 22Na.Health Physics 22(4): 387-392. 
Baxter P, Stoiber RE, Williams SN (1982). Volcanic gases and health: Masaya volcano, Nicaragua. Lancet 2: 150-151. Measuring volcanic degassing of SO2 in the lower

Berk A, Berstein SL, Robertson DC (1989) MODTRAN: a moderate resolution model for LOWTRAN-7. Air Force Geophys Lab Tech Rept GL-TR-890122, Hanscom AFB, MA.

Berresheim H, Jaeschke W (1983) The contribution of volcanoes to the atmospheric sulfur budget. Journal of Geophysical Research 88(C6): 3732-3740.

Bluth GSJ, Rose WI, Sprod IE , Krueger AJ (1997). Stratospheric Loading of Sulfur from Explosive Volcanic Eruptions. Journal of Geology 105 (6): 671-683.

Bluth GJS, Shannon JM, Watson IM, Prata AJ, Realmuto VJ (2007) Development of an Ultra-violet digital camera for volcanic $\mathrm{SO}_{2}$ imaging. Journal of Volcanology and Geothermal Research 161: 47-56.

Boichu M, Oppenheimer C, Tsanev VI, Kyle PR (2010) High temporal resolution $\mathrm{SO}_{2}$ flux measurements at Erebus volcano, Antarctica. Journal of Volcanology and Geothermal Research 190: 325-336

Brantley S, Koepenick K (1995). Measured carbon dioxide emissions from Oldoinyo Lengai and the skewed distribution of passive volcanic fluxes. Geology 23: 933-936.

Bulletin of the Global Volcanism Network (1989) Lascar, 14:11, Smithsonian Institution, Washington DC. 
Bulletin of the Global Volcanism Network (1993) Lascar, 18:08, Smithsonian Institution, Washington DC.

Bulletin of the Global Volcanism Network (1994) Lascar, 19:01, Smithsonian Institution, Washington DC.

Bulletin of the Global Volcanism Network (2000-2011) Fuego, Smithsonian Institution, Washington DC.

Bulletin of the Global Volcanism Network (2000-2010) Masaya, Smithsonian Institution, Washington DC.

Bulletin of the Global Volcanism Network (2000-2010) San Cristobal, Smithsonain Institution, Washington DC.

Bulletin of the Global Volcanism Network (2000-2007) Santa Ana, Smithsonian Institution, Washington DC.

Bulletin of the Global Volcanism Network (2000-2010) Telica, Smithsonian Institution, Washington DC.

Bulletin of the Global Volcanism Network (2000-2007) San Miguel, Smithsonian Institution, Washington DC.

Bulletin of the Global Volcanism Network (2000) Lascar, 25:06, Smithsonian Institution, Washington DC.

Bulletin of the Global Volcanism Network (2000) San Cristobal, 25:05, Smithsonian Institution, Washington DC. 
Bulletin of the Global Volcanism Network (2000) Santiaguito, 26:04, Smithsonian Institution, Washington DC.

Bulletin of the Global Volcanism Network (2001) Santa Ana, 26:04, Smithsonian Institution, Washington DC.

Bulletin of the Global Volcanism Network (2002) Fuego, 27:08, Smithsonian Institution, Washington DC.

Bulletin of the Global Volcanism Network (2002) Guangua Pichincha, 29:06, Smithsonian Institution, Washington DC.

Bulletin of the Global Volcanism Network (2002) Reventador, 27:11, Smithsonian Institution, Washington DC.

Bulletin of the Global Volcanism Network (2002) San Miguel, 20:03, Smithsonian Institution, Washington DC.

Bulletin of the Global Volcanism Network (2002) Tungurahua, 27:12, Smithsonian Institution, Washington DC.

Bulletin of the Global Volcanism Network (2003) Lascar, 28:03, Smithsonain Institution, Washington DC.

Bulletin of the Global Volcanism Network (2005) Conception, 32:07, Smithsonian Institution, Washington DC.

Bulletin of the Global Volcanism Network (2005) Lascar, 30:04 Smithsonian Institution, Washington DC. 
Bulletin of the Global Volcanism Network (2005) Pacaya, Smithsonian Institution, Washington DC.

Bulletin of the Global Volcanism Network (2005) Santa Ana, 30:09, Smithsonian Institution, Washington DC.

Bulletin of the Global Volcanism Network (2006) Lascar, 31:04, Smithsonian Institution, Washington DC.

Bulletin of the Global Volcanism Network (2006) Lascar, 31:11, Smithsonian Institution, Washington DC.

Bulletin of the Global Volcanism Network (2006) Tungurahua, 31:07, Smithsonian Institution, Washington DC.

Bulletin of the Global Volcanism Network (2006-2010) Ubinas, Smithsonain Institution, Washington DC.

Bulletin of the Global Volcanism Network (2007) Lascar, 32:09, Smithsonian Institution, Washington DC.

Bulletin of the Global Volcanism Network (2007) San Miguel, 32:09, Smithsonian Institution, Washington DC.

Bulletin of the Global Volcanism Network (2008) Conception, 33:06, Smithsonain Institution, Washington DC. 
Bulletin of the Global Volcanism Network (2008) Chaiten, Smithsonian Institution, Washington DC.

Bulletin of the Global Volcanism Network (2008) Nevado del Huila, 33:01, Smithsonian Institution, Washington DC.

Bulletin of the Global Volcanism Network (2008) Pacaya, Smithsonian Institution, Washington DC.

Bulletin of the Global Volcanism Network (2008) Pacaya, Smithsonian Institution, Washington DC.

Bulletin of the Global Volcanism Network (2010) Telica, 35:03, Smithsonain Institution, Washington DC.

Burton M., Salerno G, Caltabiano T, Condarell D, Longo V, Murè F (2003) Volcanic $\mathrm{SO}_{2}$ Emissions During the 2002/2003 Eruption of Stromboli. Eos Transactions. AGU 84 (46), Fall Meet, Abstract V12H-04.

Burton MR, Caltabiano T, Murè F, Salerno G, Randazzo D (2009) SO2 flux from Stromboli during the 2007 eruption: Results from the FLAME network and traverse measurements. Journal of Volcanology and Geothermal Research 182(3-4): 214-220.

Cadle, R. D (1975) Volcanic emissions of halides and sulfur compounds to the troposphere and stratosphere. Journal of Geophysical Research 80: 1650-1652, 1975.

Caltabiano T, Romano R, Budetta G (1994) $\mathrm{SO}_{2}$ flux measurements at Mount Etna (Sicily). Journal of Geophysical. Research, 99 (12): 809-12,819. 
Caltabian T, Burton M, Giammanco S, Allard P, Bruno N, Murè F, Romano R, (2004) Volcanic gas emissions from the summit craters and flanks of Mt. Etna, 1987-2000. In: Mt Etna Volcano Laboratory. (Eds: Bonaccorso A, Calvari S, Coltelli M, Del Negro C, Falsaperla F) GEOPHYSICAL MONOGRAPH SERIES, 143:111-128.

Campion R, Salerno GG, Coheur PF, Hurtmans D, Clarisse L, Kazahaya K, Burton M, Caltabiano T, Clerbaux C, Bernard A (2010) troposphere with ASTER band ratios. Journal of Volcanology and Geothermal Research 194(1-3): 42-54.

Carn SA, Froyd KD, Anderson BE, Wennberg P, Crounse J, Sperncer K, Dibb JE, Krotkov NA, Browell EV, Hair JW, Diskin G, Sachse G (2011) In situ measurements of tropospheric volcanic plumes in Ecuador and Colombia during TC ${ }^{4}$. Journal of Geophysical Research, 116: D00J24.

Carn SA, Krueger AJ, Bluth GJS, Schaefer SJ, Krotkov NA, Watson IM, Datta S (2003) Volcanic eruption detection by the Total Ozone Mapping Spectrometer (TOMS) instruments: a 22-year record of sulphur dioxide and ash emissions. Geological Society, London, Special Publications 213(1): 177-202.

Carn S A., Krueger AJ, Krotkov NA, Yang K (2007) Sulfur dioxide emissions from Peruvian copper smelters detected by the Ozone Monitoring Instrument. Geophysical Research Letters 34(9): L09801.

Carn S A, Krueger AJ, Arellano S, Krotkov NA, Yang K (2008) Daily monitoring of Ecuadorian volcanic degassing from space Journal of Volcanology and Geothermal Research 176(1): 141-150. 
Carn S, Lopez T (2011) Opportunistic validation of sulfur dioxide in the Sarychev Peak volcanic eruption cloud. Atmospheric Measuring Techniques, 4: 1705-1712.

Cashman KV, Mangan MT (1994) Physical aspects of magma degassing II. Constraints on vesiculation processes from textural studies of eruptive products. Reviews in Mineralogy 30:447-478.

Chesner CA, Rose WI (1984) Geochemistry and evolution of the Fuego volcanic complex, Guatemala. Journal of Volcanology and Geothermal Research 21:25-44.

Crisp J, Baloga S (1990) A model for lava flows with two thermal components. Journal of Geophysical Research, Solid Earth, 95, B2: 1255-1270.

Dalton MP, Watson IM, Nadeau PA, Werner C, Morrow W, Shannon JM (2009) Assessment of the UV camera sulfur dioxide retrieval for point source plumes. Journal of Volcanology and Geothermal Research 188: 358-366.

Dalton MP, Waite GP, et al. (2010). Multiparameter quantification of gas release during weak Strombolian eruptions at Pacaya Volcano, Guatemala. Geophysical Research Letters 37(9): L09303.

Dean K, Bowling SA, Shaw G, Tanaka H (1994) Satellite analyses of movement and characteristics of the Redoubt volcano plume, January 8, 1990. Journal of Volcanology and Geothermal Research 62: 339352.

De La Cruz Martınez V, Hernandez Zuniga R (1986). Geologia del Volcan Tacana. Geotermia: Revista Mexicana de Geoenergia 2 (1). 
Delmelle P (2003) Environmental impacts of tropospheric volcanic gas plumes. Geological Society, London, Special Publications 213(1): 381-399.

Delmelle P, Stix J, Bourgue HA, Baxter PJ, Alvarez JG, Barquero J (2001) Dry deposition and heavy acid loading in the vicinity of Masaya volcano, a major sulfur and chlorine source in Nicaragua. Environmental Science and Technology 7: 1289-1293.

Delmelle P, Stix J, Baxter P, Garcia-Alvarez J, Barquero J (2002) Atmospheric dispersion, environmental effects and potential health hazard associated with the low-altitude gas plume of Masaya volcano, Nicaragua. Bulletin of Volcanology 64: 423-434.

Delmelle P, Stix J (2000) Volcanic Gases. In: Encyclopedia of Volcanoes (eds. H. Sigurdsson B. Houghton S. McNutt H. Rymer J. Stix), Academic Press, San Diego: 803-815.

DeMets C (2001) A new estimate for present day Cocos Caribbean Plate motion: Implications for slip along the Central American Volcanic Arc. Geophysical Research Letters 28(21): 4043-4046.

Dozier J (1981) A method for satellite identification of surface temperature fields of subpixel resolution. Remote Sensing of Environment, 11: 221-229.

\section{Eatough D J, Caka FM, Farber RJ (1994) The conversion of SO2} to sulfate in the atmosphere, Israel Journal of Chemistry, 34: 301-314.

Edmonds M, Herd RA, Galle B, Oppenheimer CM (2003) Automated high time resolution measurements of $\mathrm{SO}_{2}$ flux at Soufriere Hills Volcano, Montserrat. Bulletin of Volcanology 65: 578-586. 
Ellrod GP, Connell BH, Hilliger DW (2003) Improved detection of airborne volcanic ash using multispectral infrared satellite data. Journal of Geophysical Research 198, D12: 4356.

Ellrod GP (2004) Impact on volcanic ash detection caused by the loss of the $12.0 \mu \mathrm{m}$ "Split Window" band on GOES Imagers. Journal of Volcanology and Geothermal Research 135: 91-103.

Francis P, Rothery D (1987) Using the Landsat Thematic Mapper to detect and monitor active volcanoes: An example from Lascar volcano, northern Chile. Geology 15: 614-617.

Friend J P, Bandy AR, Moyers JL, Zoller WH, Stoiber RE, Torres AL, Rose WI, McCormick MP, Woods DC (1982) Research on atmospheric volcanic emissions: An overview. Geophysical Research Letters 9(9): 1101-1104.

Galle B, Oppenheimer C, Geyer A, McGonigle A, Edmonds M, Horrocks LA (2002) A miniaturized ultraviolet spectrometer for remote sensing of $\mathrm{SO}_{2}$ fluxes: a new tool for volcano surveillance. Journal of Volcanology and Geothermal Research 119: 241-254.

Galle B, Johansson M, Rivera C, Zhang Y, Kihlman M, Kern C, Lehmann T, Platt U, Arellano S, Hidalgo S (2010) Network for Observation of Volcanic and Atmospheric Change (NOVAC)- A global network for volcanic monitoring: Network layout and instrument description. Journal of Geophysical Research, 115: D05304.

Gardeweg MC, Medina E (1994) La erupcione subpliniana del 19-20 Abril de 1993 del Volcan Lascar, N.de Chil. Acta $7^{\text {th }}$ Congr Geol Chileno 7:299304. 
Gardeweg MC, Sparks RSJ, Matthews SJ (1998) Evolution of Lascar volcano, northern Chile. Journal of the Geological Society London 155: 89-104.

Gillespie AR (1992) Enhancement of Multispectral Thermal Infrared Images: Decorrelation Contrast Stretching. Remote Sensing of the Environment 42: 137-145.

Gillespie A, Rokugawa S, Matsunaga T, Cothern S, Hook S, Kahle (1998) A Temperature and emissioty speration algorithm for the Advanced Spaceborne Thermal Emission and Reflection Radiometer (ASTER) images. IEEE Transactions on Geoscience and Remote Sensing 36 (4):1113-1126.

Glaze LS, Francis PW, Self S, Rothery DA (1989a) The 16 September 1986 eruption of Lascar Volcan, north Chile: satellite investigations. Bulletin of Volcanolgy 51:149-160.

Glaze LS, Francis PW, Rothery DA (1989b) Measuring thermal budgets of active volcanoes by satellite remote sensing. Nature 338: 144-146.

Global Sulfur Dioxide Monitoring Home Page (2010-) http://so2.gsfc.nasa.gov/, National Aeronautics and Space Administration.

Global Volcanism Program, Arenal Summary.

Global Volcanism Program, Cerro Negro Summary.

Global Volcanism Program, Conception, Summary.

Global Volcanism Program, Copahue Summary. 
Global Volcanism Program, El Misti Summary.

Global Volcanism Program, Galeras Summary.

Global Volcanism Program, Irazu Summary.

Global Volcanism Program, Irruputuncu Summary.

Global Volcanism Program, Lastarria Summary.

Global Volcanism Program, Llaima Summary.

Global Volcanism Program, Nevado del Huila.

Global Volcanism Program, Poas Summary.

Global Volcanism Program, Turrialba Summary.

Global Volcanism Program, Sangay Summary.

Global Volcanism Program, Villarrica Summary.

Global Volcanism Program, Ubinas Summary.

Graf HF, Feichter J. Langmann B (1997) Volcanic sulfur emissions: Estimates of source strength and its contribution to the global sulfate distribution. Journal of Geophysical Research 102(D9): 10727-10738. 
Halmer MM, Schmincke HU, Graf HF (2002) The annual volcanic gas input into the atmosphere, in particular into the stratosphere: a global data set for the past 100 years. Journal of Volcanology and Geothermal Research 115(3-4): 511-528.

Henney (2006) The use of the Advanced Spacebourne Thermal Emission and Reflection Radiometer. MSc thesis, University of Bristol.

Hilliger DW, Clark JD (2002) Principal Component Image Analysis for Volcanic Ash. Part I: Most Important Bands and Implications for Future GOES Imagers. Journal of Applied Meteorology 41: 985-1001.

Holasek, RE, Self S (1995) GOES weather satellite observations and measurements of the May 18, 1980, Mount St. Helens eruption, Journal of Geophysical. Research, 100: 8469-8487.

Holland ASP, Watson I M, Phillips JC, Caricchi L, Dalton MP (2011) Degassing processes during lava dome growth: Insights from Santiaguito lava dome, Guatemala. Journal of Volcanology and Geothermal Research 202(1-2): 153-166.

Horrocks L A, Oppenheimer C, Burton M, Duffell H (2003) Compositional variation in tropospheric volcanic gas plumes: evidence from groundbased remote sensing. Geological Society, London, Special Publications 213(1): 349-369.

Instituto Colombiano de Geologia y Mineria (INGEOMINAS) www.INGEOMINAS.gov.co (2010). 
Jensen JR (2005) Introductory Digital Image Processing: A Remote Sensing Perspective. Third Edition: Prentice Hall.

Kellogg WW, Cadle RD, Allen ER, Lazrus AL, Martell A (1972) The Sulfur Cycle. Science 175(4022): 587-596.

Kristiansen NI, Stohl A, Prata AJ, Richter A, Eckhardt S, Seibert P, Hoffmann A, Ritter C, Bitar L, Duck TJ, Stebel K (2010) Remote sensing and inverse transport modeling of the Kasatochi eruption sulfur dioxide cloud. JOURNAL OF Geophysical Research, 115: D00L16

Krueger AJ, Walter LS, Bhartia PK, Schnetzler CC, Krotkov NA, Sprod I, Bluth GJS (1995) Volcanic sulfur dioxide measurements from the total ozone mapping spectrometer instruments. Journal of Geophysical Research, 100 (D7): 14,057-14,076.

Krotkov NA., Carn SA, Krueger AJ, Bhartia PK, Yang K (2006) Band residual difference algorithm for retrieval of $\mathrm{SO}_{2}$ from the aura ozone monitoring instrument (OMI). Geoscience and Remote Sensing, IEEE Transactions 44(5): 1259-1266.

Land Processes Distributed Active Archive Center (LP DAAC) (2012) https://lpdaac.usgs.gov/.

Lambert G, Le Cloarec MF, Pennisi M (1988) Volcanic output of SO2 and trace metals: A new approach. Geochimica et Cosmochimica Acta 52(1): 3942.

Le Guern F (1982) Les debitsd e CO2 et de SO2 volcaniquesd ans l'atmosph•re, Bulletin of Volcanology 45: 197-202. 
Malinconico LL (1987) On the variation of $\mathrm{SO}_{2}$ emission from volcanoes. Journal of Volcanology and Geothermal Research, 33: 231-237.

Marrett R, Allmendinger R (1991) Estimates of strain due to brittle faulting: sampling of fault populations. Journal of Structural Geology, 13: 735-738.

Mather TA, Allen AG, Oppenheimer C, Pyle DM, McGonigle AJS (2003) Sizeresolved characterisation of soluble ions in the particles in the tropospheric plume of Masaya volcano, Nicaragua: origins and plume processing. Journal of Atmospheric Chemistry 46(3): 207.

Mather TA, Tsanev V I, Pyle DM, McGonigle AJS, Oppenheimer C, Allens AG (2004) Characterization and evolution of tropospheric plumes from Lascar and Villarrica volcanoes, Chile. Journal of Geophysical Research 109(D21): D21303.

Mather TA, Pyle, Tsanev VI, McGonigle AJS, Oppenheimer C, Allens AG (2006) A reassessment of current volcanic emissions from the Central American arc with specific examples from Nicaragua. Journal of Volcanology and Geothermal Research 149(3-4): 297-311.

Matthews SJ, Jones AP, Gardeweg MC (1994b) Lascar volcano, Northern Chile: evidence for steady state disequilibrium. Journal of Petrology: 401432.

Mathews SJ, Gardeweg MC, Sparks RSJ (1997) The 1984 to 1996 cyclic activity of Lascar volcano, northern Chile: cycles of dome growth, dome subsidence, degassing and explosive eruptions. Bulletin of Volcanology 59 (1):72-82. 
McCarthy EB, Bluth GJS, Watson IM, Tupper A (2008) Detection and analysis of the volcanic clouds associated with the 18 and 28 August 2000 eruptions of Miyakejima Volcano, Japan. International Journal of Remote Sensing 29: 6597-6620.

McCormick B, Mather T, Carn S (2012) First synoptic analysis of volcanic degassing in Papua New Guinea. Geochemistry Geophysics Geosystems,13:1525-2027.

McGonigle AJS, Oppenheimer C, Hayes AR, Galle B, Edmonds M, Caltabiano T, Salerno G, Burton M, Mather, TA (2003) Sulphur dioxide fluxes from Mount Etna, Vulcano, and Stromboli measured with an automated scanning ultraviolet spectrometer. Journal of Geophysical Research 108(B9): 2455.

McGonigle AJS, Oppenheimer C (2003) Optical sensing of volcanic gas and aerosol emissions. In: Oppenheimer C, Pyle DM, Barclay J, (Eds) Volcanic Degassing. Geological Society Publication 213.

McGonigle AJS, Delmelle P, Oppenheimer C, Tsanev VI, Delfosse T, WilliamsJones G, Horton K, Mather TA (2004) $\mathrm{SO}_{2}$ depletion in tropospheric volcanic plumes. Geophysical Research Letters 31(13): L13201.

Millan M (1980) Remote sensing of air pollutants. A study of some atmospheric scattering effects. Atmospheric Environment, 14(11): 1241-1253.

Mori T, Burton M (2006) The $\mathrm{SO}_{2}$ camera: A simple, fast and cheap method for ground-based imaging of $\mathrm{SO}_{2}$ in volcanic plumes. Geophysical Research Letters 33: 24 L24804. 
Murphy SW, de Souza Filho CR, Oppenheimer C (2011) Monitoring volcanic thermal anomalies from space: Size matters. Journal of Volcanology and Geothermal Research 203:48-61.

Nadeau PA, Williams-Jones G (2009) Apparent downwind depletion of volcanic $\mathrm{SO}_{2}$ : Lessons from Masaya Volcano, Nicaragua, Bulletin of Volcanology, 71(4), 389-400.

NASA Jet Propulsion Labrartory, The Advanced Thermal Emission and Reflection Spectrometer. http://asterweb.jpl.nasa.gov/eos.asp

Newhall CG, Self S (1982) The Volcanic Explosivity Index (VEI) An Estimate of Explosive Magnitude for Historical Volcanism. Journal of Geophysical Research_87(C2): 1231-1238.

Oppenheimer C, Francis P, Rothery D, Carlton R, Glaze L (1993) Infrared image analysis of volcanic features: Lascar volcano, Chile, 1984-1992. Journal of Geophysical Research:4269-4286.

Oppenheimer C, Francis P, Stix J, (1998) Depletion rates of $\mathrm{SO}_{2}$ in tropospheric volcanic plumes. Geophsyical Research Letters, 25: 2671-2674.

Oppenheimer C (1998) Volcanological applications of meteorological satellites. International Journal of Remote Sensing 19(15): 2829-2864.

Palma J L, Calder ES, Basualto D, Blake S, Rothery DA (2008) Correlations between SO2 flux, seismicity, and outgassing activity at the open vent of Villarrica volcano. Journal of Geophysical Research 113(B10): B10201. 
Pieri D, Abrams M (2004) ASTER watches the world's volcanoes: a new paradigmfor volcanological observations from orbit. Journal of Volcanology and Geothermal Research, 135: 13-28.

Platt U (1994) Differential optical absorption spectroscopy (DOAS). In: Sigrist, M. W. (Ed.), Air monitoring by spectroscopic techniques. Chemical Analysis Series, 127, 27-84. John Wiley \& Sons Inc.

Prather M (1992) Catastrophic loss of stratospheric ozone in dense volcanic clouds. Journal of Geophysical Research 97(10) 187-191.

Protti M, Güendel F, McNally K. (1995) Correlation between the age of the subducting Cocos plate and the geometry of the Wadati-Benioff zone under Nicaragua and Costa Rica: in Mann P (ed) Geological Society of America Special Paper, No. 295, Geologic and Tectonic Development of the Caribbean Plate Boundary in Southern Central America :309-326.

Ramsey M, Dehn J (2004) Spaceborne observations of the 2000 Bezymianny, Kamchatka eruption: the integration of high-resolution ASTER data into near real-time monitoring using AVHRR. Journal of Volcanology and Geothermal Research 135(1-2): 127-146.

Realmuto VJ (1990) Sperating the effects of temperature and emissivity: Emissivity spectrum normalization. $2^{\text {nd }}$ TIMS Workshop, Pasadena, CA: Jet Propulsion Lab, JPL Publication 90-55.

Realmuto VJ (2000) The potential use of earth observing system data to monitor the passive emission of sulfur dioxide from volcanoes. In: Mouginis-Mark, PJ, Crisp, JA, Fink, JH (Eds.), Remote Sensing of Active 
Volcanism. American Geophysical Union, Washington, DC, pp. 101115.

Realmuto V J, Abrams M J, Buongiorno MF, Pieri DC (1994) The use of multispectral thermal infrared image data to estimate the sulfur dioxide flux from volcanoes: A case study from Mount Etna, Sicily, July 29, 1986. Journal of Geophysical Research 99(B1): 481-488.

Realmuto VJ, Sutton A J, Elias T (1997) Multispectral thermal infrared mapping of sulfur dioxide plumes: A case study from the East Rift Zone of Kilauea Volcano, Hawaii. Journal of Geophysical Research 102(B7): 1505715072.

Realmuto VJ, Worden HM (2000) Impact of atmospheric water vapor on the thermal infrared remote sensing of volcanic sulfur dioxide emissions: A case study from the Pu'u 'O'o vent of Kilauea Volcano Hawaii. Journal of Geophysical Research 105: 21497-21508.

Reverb ECHO (2012) http://reverb.echo.nasa.gov/reverb/, National Aeronautics and Space Administration.

Robock A (2000) Volcanic eruptions and climate. Reviews of geophysics 38: 191-219.

Rodríguez LA (2007) Advances in gas and aerosol monitoring at active volcanoes, Ph.D. Dissertation, Michigan Technological University, 277 p.

Rodríguez L A, Watson IM, Rose WI, Branan YK, Bluth GJS, Chinga G, Matias O, Escobar D, Carn SA, Fischer TP (2004) SO2 emissions to the atmosphere from active volcanoes in Guatemala and El Salvador, 1999- 
2002. Journal of Volcanology and Geothermal Research 138(3-4): 325344.

Rodríguez LA, Watson IM, Edmonds M, Ryan G, Hards V, Oppenheimer CMM, Bluth GJS (2008). SO2 loss rates in the plume emitted by Soufrière Hills volcano, Montserrat. Journal of Volcanology and Geothermal Research 173(1-2): 135-147.

Rose WI, Mercado R (1986) Report on UNDRO/OFDA mission to Tacana' Volcano, Guatemala/Mexico: INSIVUMEH, Guatemala. 30 pp. +3 maps (in English or Spanish).

Rose WI, Gu Y, Watson IM, Yu T, Bluth GSJ, Prata AJ, Krueger AJ, Krotkov N, Carn S, Fromm MD, Hunton DE, Ernst GGJ, Viggiano AA, Miller TM, Ballenthin OJ, Reeves JM, Wilson JC, Anderson BE, Flittner DE (2003)The February-March 2000 eruption of Hekla, Iceland from a satellite perspective. Volcanism and the Earth's Atmosphere, Geophysical Monograph, 139:107-132

Rose S, Ramsey M (2009) The 2005 eruption of Kliuchevskoi volcano: Chronology and processes derived from ASTER spacebourne and field data. Journal of Volcanology and Geothermal Research 184: 367-380.

Rothery DA, Francis PW, Wood PA (1988) Volcano monitoring using short wavelength infrared data from satellites. Journal of Geophysical Research 93: 7993-8008.

Salerno GG, Burton MR, Oppenheimer C, Caltabino T, Randazzo D, Bruno N, Longo (2009) Three-years of SO2 flux measurements of Mt. Etna using an automated UV scanner array: Comparison with conventional 
traverses and uncertainties in flux retrieval. Journal of Volcanology and Geothermal Research 183: 76-83.

Sanchez Bennett EH, Rose WI, Conway FM (1992) Santa Marıa, Guatemala: a decade volcano. Eos Transactions -American Geophysical Union 73, $521-522$.

Sawyer GM, Salerno GG, Le Blond JS, Martin RS, Spampinato L, Robers TJ, Mather TA, Witt MLI, Tsanev VI, Oppenheimer C (2011) Gas and aerosol emissions from Villarrica volcano, Chile. Journal of Volcanology and Geothermal Research 203(1-2): 62-75.

Schneider DJ, Rose WI, Coke LR, Bluth GJS (1999) Early evolution of a stratospheric volcanic eruption cloud as observed with TOMS and AVHRR. Journal of Geophysical Research, 104 (D4): 4037-4050

Schnetzler, CC, Bluth GJS, Krueger AJ, Walter LS (1997) A proposed volcanic sulfur dioxide index (VSI). Journal of Geophysical Research 102(B9): 20087-20091.

Scientific Event Alert Network Bulletin (1987) Tacana, Smithsonain Institution, Washington DC.

Simkin T, Siebert L (2002-). Global Volcanism, Smithsonian Institution, Global Volcanism Program Digital Information Series, GVP-5 (http://www.volcano.si.edu).

Sparks RSJ (1997) Causes and consequences of pressurization in lava dome eruptions. Earth and Planetary Letters150: 177-189. 
Sparks RSJ (2003) Dynamics of Degassing. In Oppenheimer C, Pyle DM and Barclay,J (Eds.), Volcanic Degassing, Geological Society, London, Special Publication 213: 5-22.

Spinei E, Carn S, Krotkov NA, Mount GH, Yang K, Krueger A (2010) Validation of ozone monitoring instrument SO2 measurements in the Okmok volcanic cloud over Pullman, WA, July 2008. Journal of Geophysical Research,115: D00L08.

Steffke A M, Harris AJL, Burton M, Caltabiano T, Salerno,G.G (2011) Coupled use of COSPEC and satellite measurements to define the volumetric balance during effusive eruptions at Mt. Etna, Italy. Journal of Volcanology and Geothermal Research 205(1-2): 47-53.

Stenchikov GL, Kirchner I, Robock A, Graf HF, Autuna R, Grainger A, Lambert A, Thomason L (1998) Radiative forcing from the 1991 Mount Pinatubo volcanic eruption. Journal of Volcanology and Geothermal Research 103 (13): 837-857.

Stoiber RE, Jepsen A (1973) Sulfur Dioxide Contributions to the Atmosphere by Volcanoes. Science 182(4112): 577-578.

Stoiber RE, Williams SN, Huebert B (1987) Annual contribution of sulfur dioxide to the atmosphere by volcanoes. Journal of Volcanology and Geothermal Research 33(1-3): 1-8.

Stoiber RE, Malinconico LL, Williams SN, (1983) Use of the Correlation spectrometer at volcanoes. In Tazieff H, Sabroux JC (Eds) Forecasting Volcanic Events. Elsevier, Amesterdam: 425-444. 
Symonds RB, Rose WI, Bluth G, Gerlach TM, (1994) Volcanic gas studies: methods, results, and applications. In Carroll, MR, and Holloway JR, (Eds), Volatiles in Magmas: Mineralogical Society of America Reviews in Mineralogy, 30: 1-66.

Tassi F, Aguilera F, Vaselli O, Medina E, Tedesco D, Delgado Huertas R, Kojima S (2009) The magmatic and hydrothermal dominated fumarolic system at the Active Crater of Lascar volcano, northern Chile. Bulletin of Volcanology 71: 171-183./]93s7

Tassi F, Aguilera F, Vaselli O, Darrah T, Medina E (2011) Gas discharges from four remote volcanoes in northern Chile: a geochemical survey. Annals of Geophysics 55: 5173.

Thomas HE, Watson IM (2010) Observations of volcanic emissions from space: current and future perspectives. Natural Hazards 54(2): 323-323-354.

Thomas HE, Watson IM, Kearney C, Carn SA, Murray SJ (2009) A multi-sensor comparison of sulphur dioxide emissions from the 2005 eruption of Sierra Negra volcano, Galápagos Islands. Remote Sensing of Environment 113(6): 1331-1342.

Varekamp JC, Ouimette A, Herman SD, Delpino, Bermudez A (2001)The 1990-2000 eruptions of Copahue,Argentina: a "bee-hive volcano"in Turmoil. Geology,29: 1059-1062.

Varekamp JC, Maarten deMoor J, Merrill MD, Colvin AS, Goss AR, Vroon PS, Hilton DR, (2006) The Geochemistry and Isotopic Characteristics of the Caviahue Copahue Volcanic Complex, Province of Neuquen,Argentina. GSA Special Paper, 407:317-342. 
Vaughan RG, Hook SJ (2006) Using satellite data to characterize the temporal thermal behavior of an active volcano: Mt St Helens, WA. Geophyscical Research Letters 33 L20303.

Vaughan RG, Keszthelyi LP, Davies AG, Schneider DJ, Jaworowski C, Heasler $H$ (2010) Exploring the limits of identifying sub-pixel thermal features using ASTER TIR data. Journal of Volcanology and Geothermal Research 189 (3-9): 225-237.

Vita F, Inguaggiato S, Bobrowski N, Calderone L, Galle B, Parello F (2012) Continuous $\mathrm{SO}_{2}$ flux measurements from Volcano Island, Italy. Annals of Geophysics, 55:301-308.

Watson IM, Realmuto VJ, Rose WI, Prata AJ, Bluth GJS, Gu Y, Bader CE, Yu T (2004) Thermal infrared remote sensing of volcanic emissions using the moderate resolution imaging spectroradiometer, Journal of Volcanology and Geothermal Research 135: 75-89.

Weibring P, Edner H, Svanberg S, Cecchi G, Pantani L, FerraraR, Caltabiano $T$ (1998) Monitoring of volcanic sulphur dioxide emissions using differential absorption lidar (DIAL), differential optical absorption spectroscopy (DOAS), and correlation spectroscopy (COSPEC). Applications in Physics, 67: 419-426.

Weibring P, Swartling J, Edner H, Svanberg S, Caltabiano T, Condarelli D, Cecchi G, Pantani L (2002) Optical monitoring of volcanic sulphur dioxide emissions - comparison between four different remote-sensing spectroscopic techniques. Optical Lasers Engineering, 37: 267-284. 
Williams-Jones G, Horton K, Elias T, Garbeil H, Mouginis-Mark PJ, Sutton AJ, Harris AJL (2006) Accurately measuring volcanic plume velocity with multiple UV spectrometers. Bulletin of Volcanology 68(4): 328-332.

Williams-Jones, Stix J, Hickson C (2008) The COSPEC Cookbook: Making $\mathrm{SO}_{2}$ Gas Measurements at Active Volcanoes IAVCEI Methods in Volcanology 1. IAVCEI.

Witt MLI, Mather TA, Pyle DM, Aiuppa A, Bagnato E, Tsanev (2008) Mercury and halogen emissions from Masaya and Telica volcanoes, Nicaragua. Journal of Geophysical Research, 113: JD005401.

Witter J B, Kress VC, Delmelle P, Stix J (2004) Volatile degassing, petrology, and magma dynamics of the Villarrica Lava Lake, southern Chile. Journal of Volcanology and Geothermal. Research 134: 303-337.

Wright R, Flynn L (2003) On the retrieval of lava flow surface temperatures from infrared satellite data. Geology, 31: 893-896.

Wooster MJ, Rothery DA (1997) Thermal Monitoring of Lascar Volcano, Chile, using infrared along track scanning radiometer: a 1992-1995 time series. Bulletin of Volcanology 58, 7: 566-579.

Wooster MJ (2001) Long term infrared surveillance of Lascar Volcano: contrasting activity cycles and cooling pyroclastics. Geophysical Research Letters 28 (5): 847-850.

Urai M (2004) Sulfur dioxide flux estimation from volcanoes using Advanced Spaceborne Thermal Emission and Reflection Radiometer - a case study 
of Miyakejima volcano, Japan. Journal of Volcanology and Geothermal Research 134: 1-13.

United States Geological Survey (2011) http://www.usgs.gov.

Yamaguchi Y, Kahle AB, Pniel M, Tsu H, Kawakami T (1998) Overview of Advanced Spaceborne Thermal Emission and Reflection Radiometer (ASTER). IEEE Transactions on Geoscience and Remote Sensing, 36(4): 1062-1071.

Yang K, Krotkov NA, Krueger AJ, Carn SA, Bhartia PK, Levelt PF (2007) Retrieval of large volcanic SO2 columns from the Aura Ozone Monitoring Instrument: Comparison and limitations. Journal of Geophysical Research, 112, D24S43, doi:10.1029/2007JD008825.

Zimmer MM, T P Fischer T P, Hilton DR, Alvarado GE, Sharp ZD, Walke JA (2004). Nitrogen systematics and gas fluxes of subduction zones: Insights from Costa Rica arc volatiles. Geochemistry Geophysics Geosystems 5(5): Q05J11.mo 
Appendix A

Factor Loadings from Chapter 2 


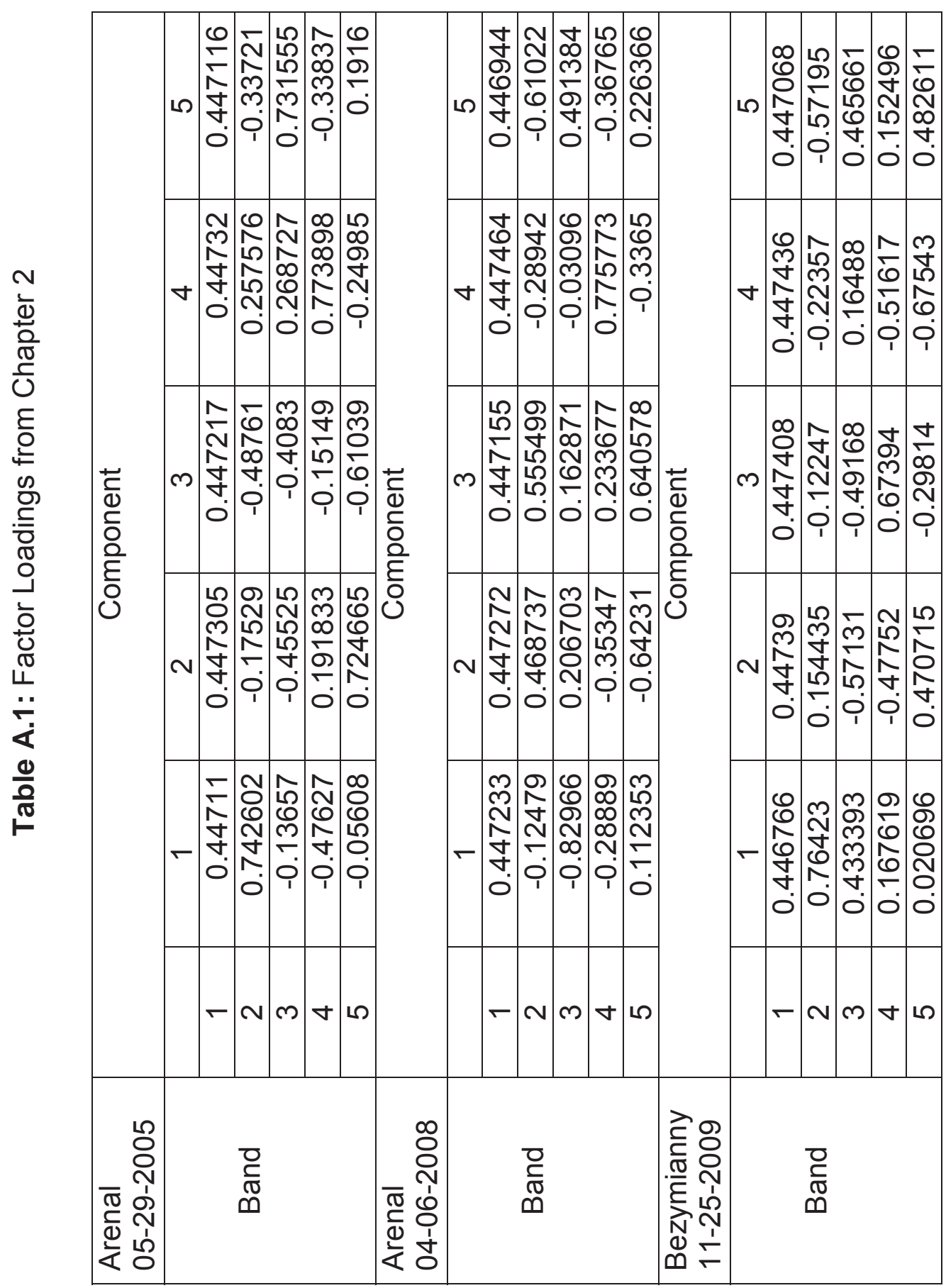




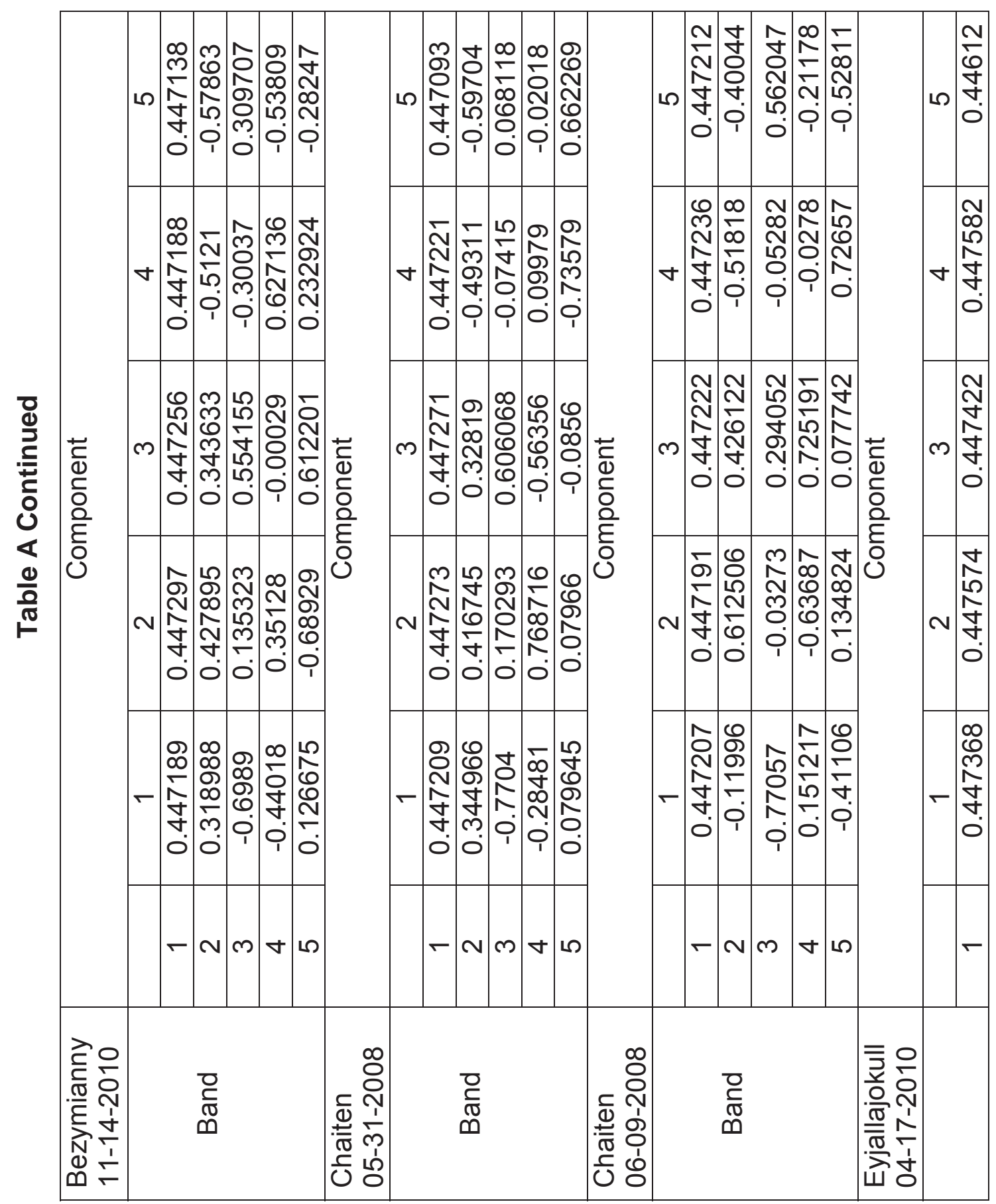




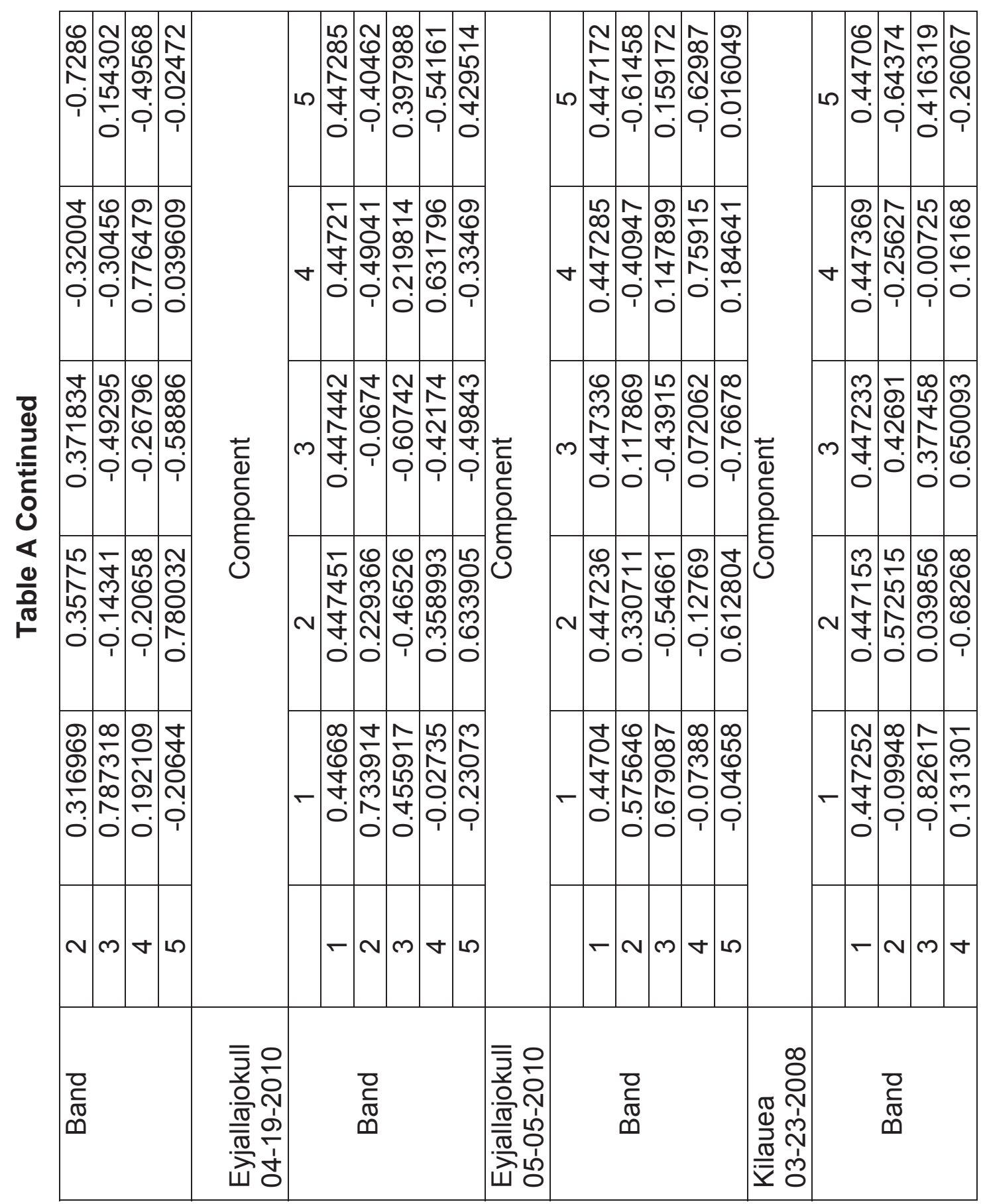




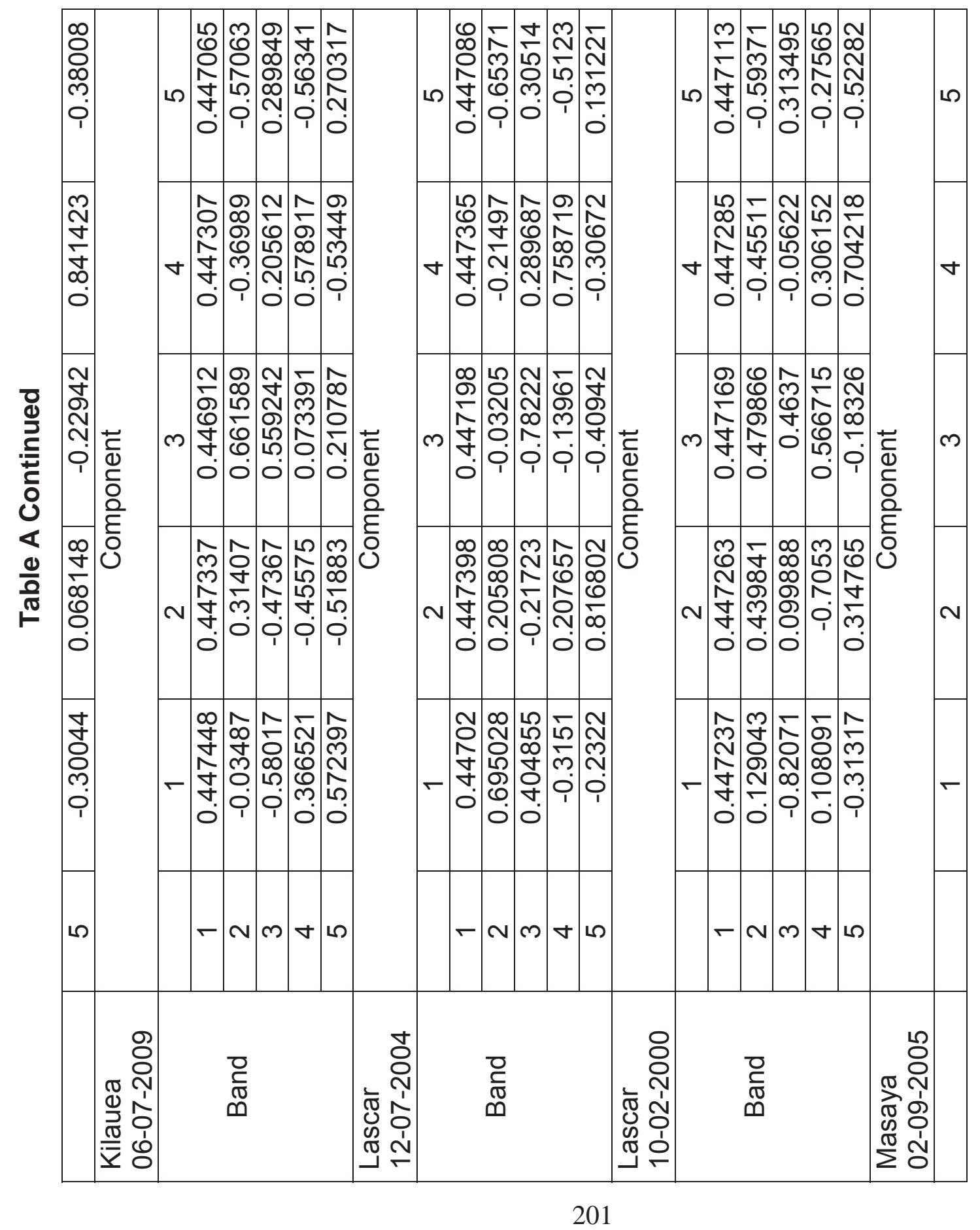




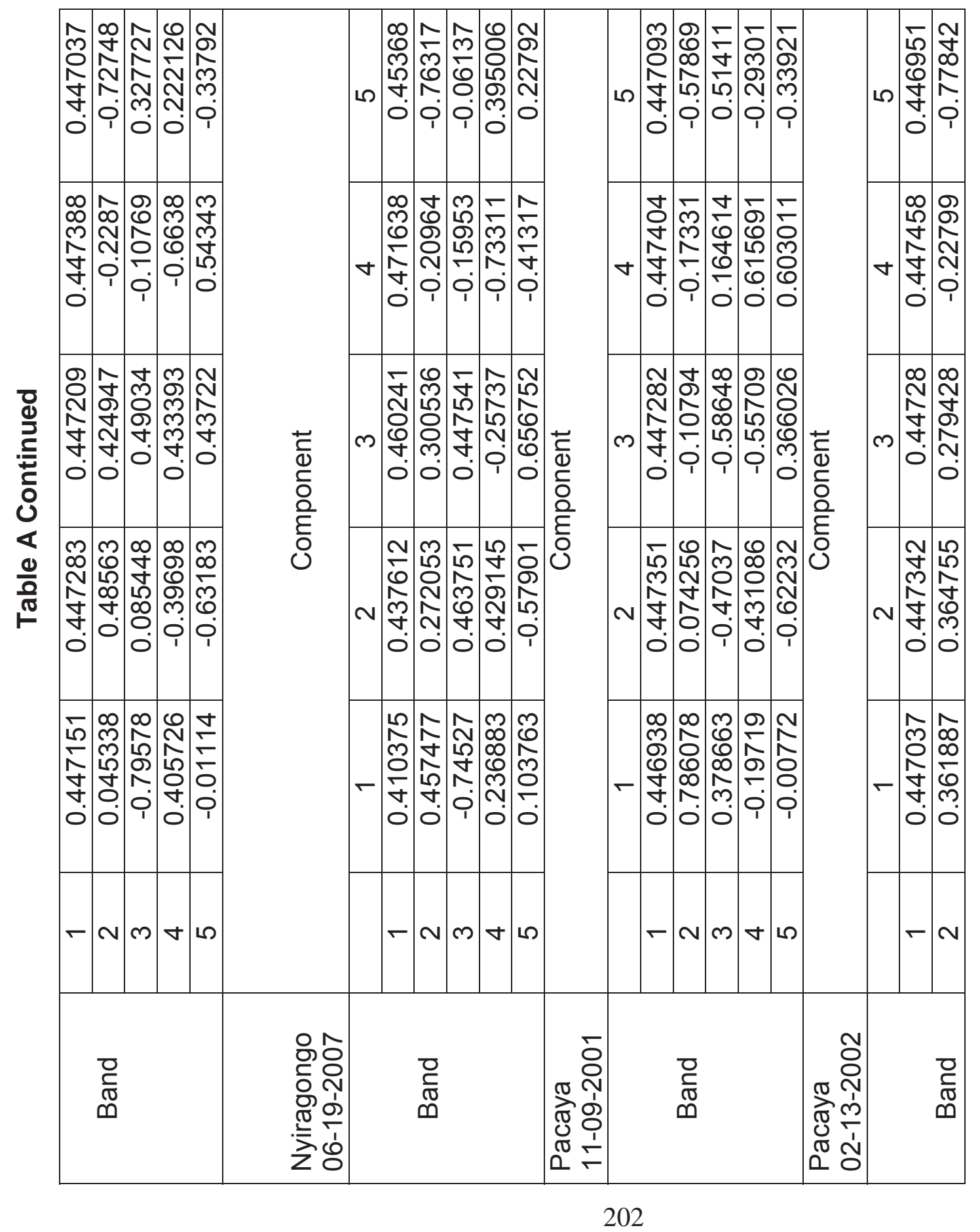




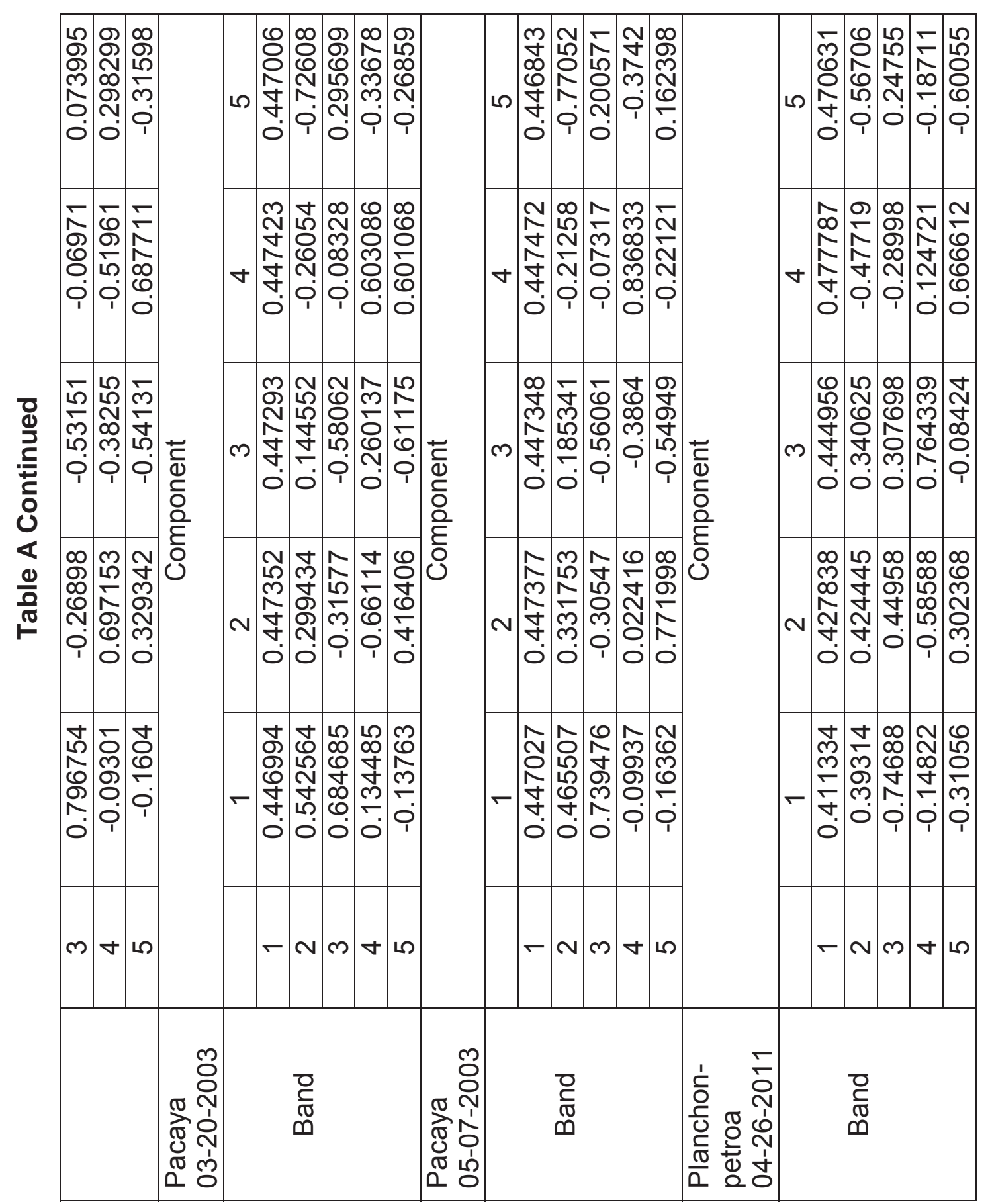




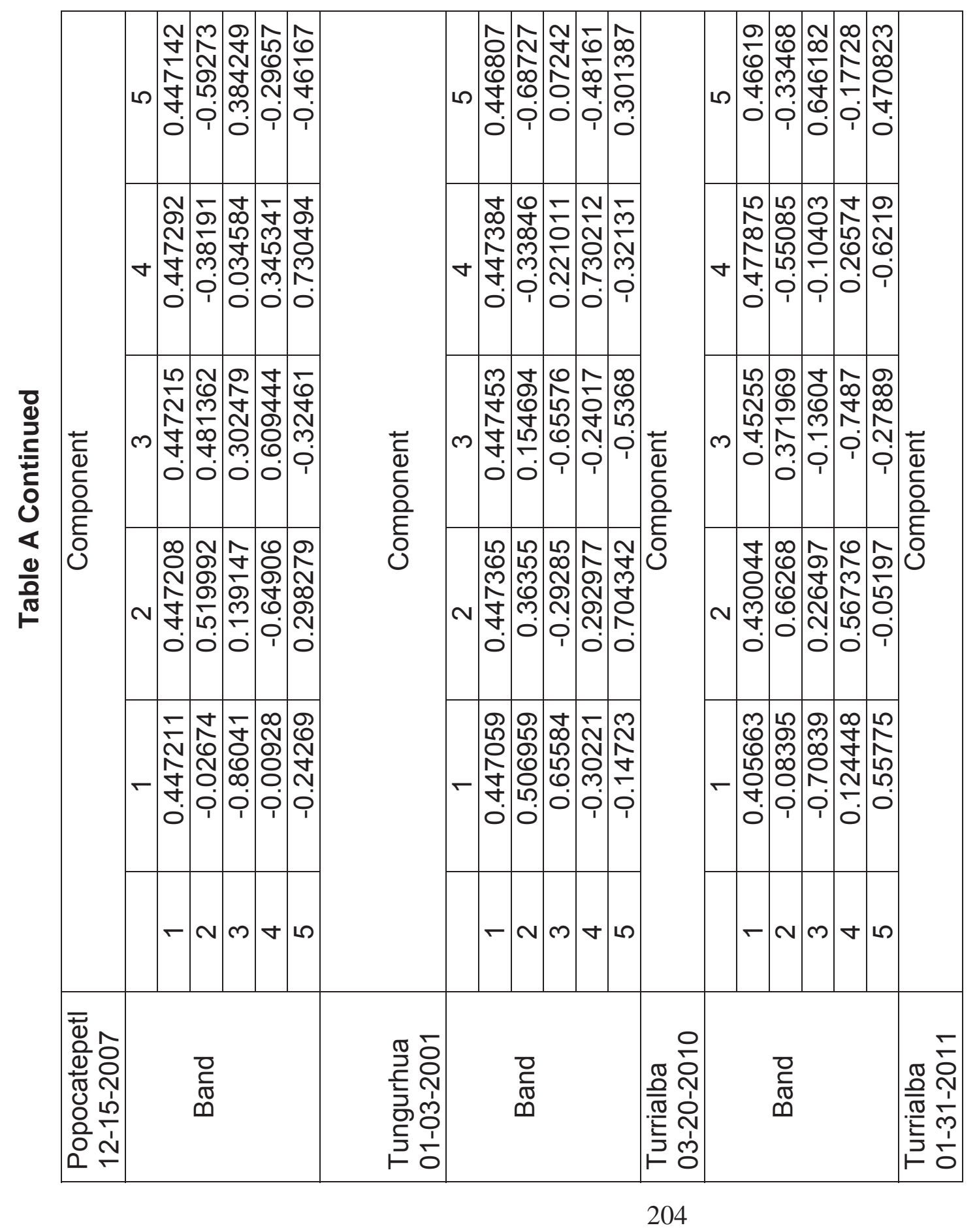




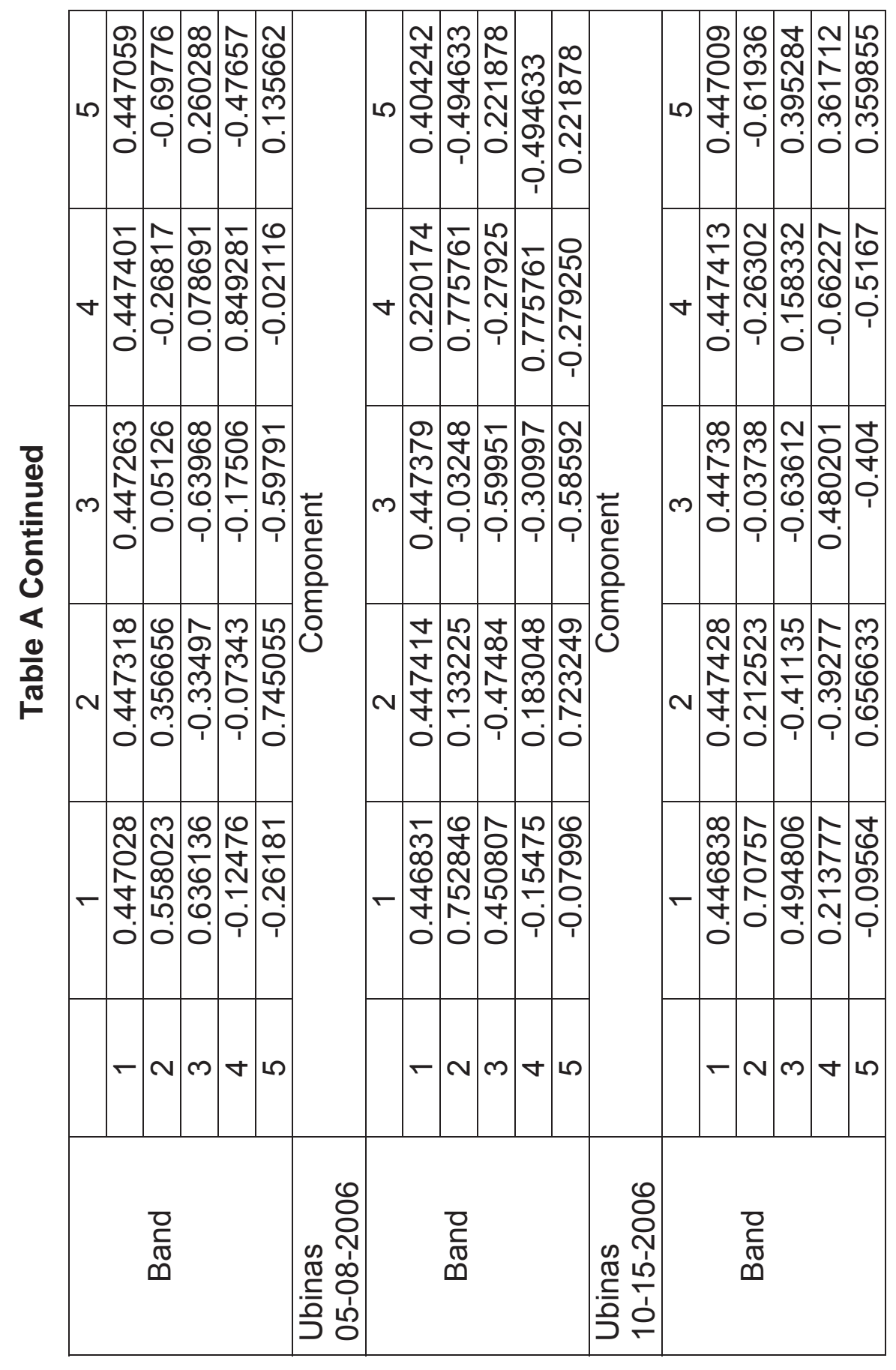




\section{Appendix B}

PCA and DCS images from Chapter 3 

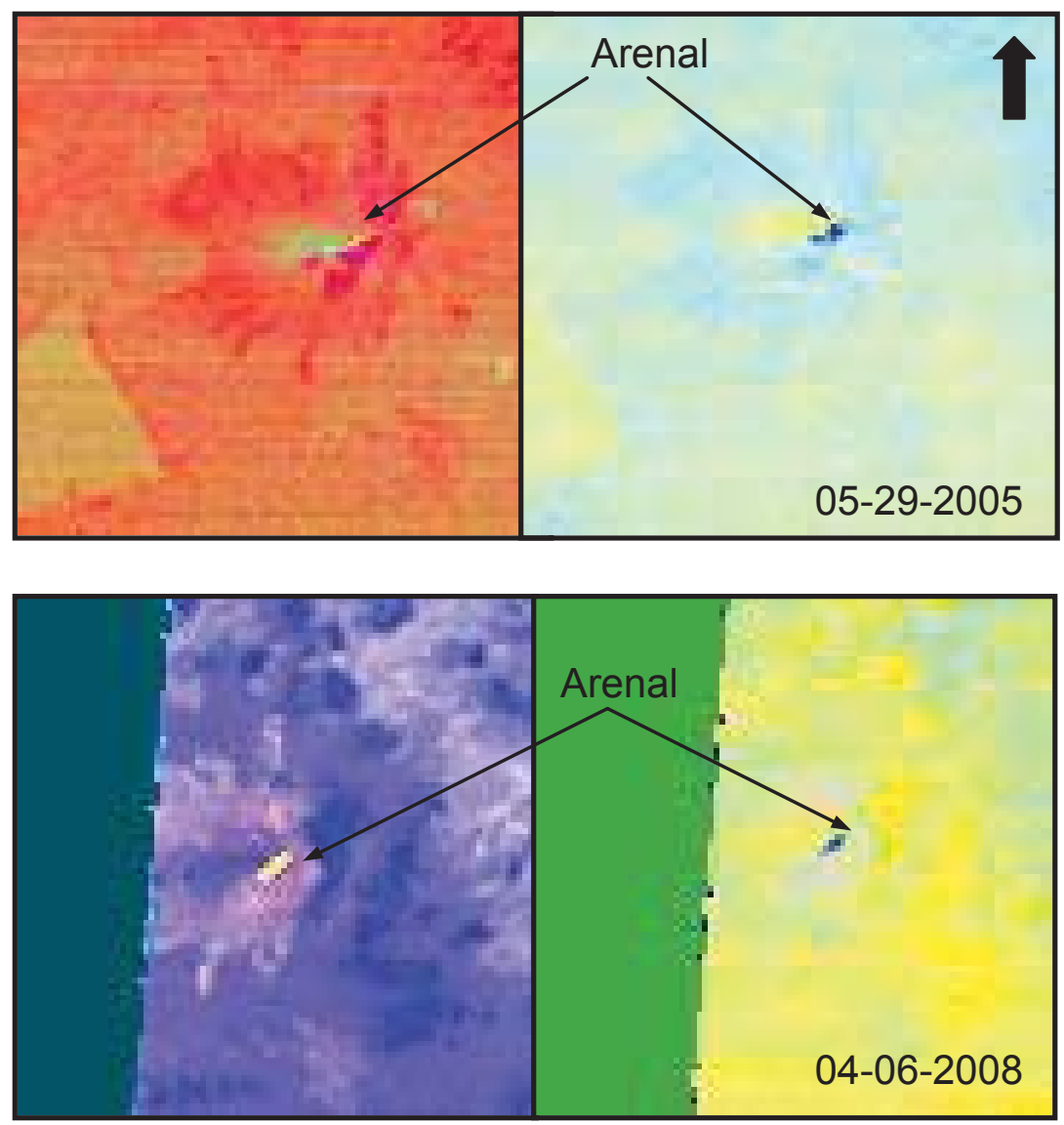

Figure B1: PCA and DCS enhancements of Arenal, Costa Rica

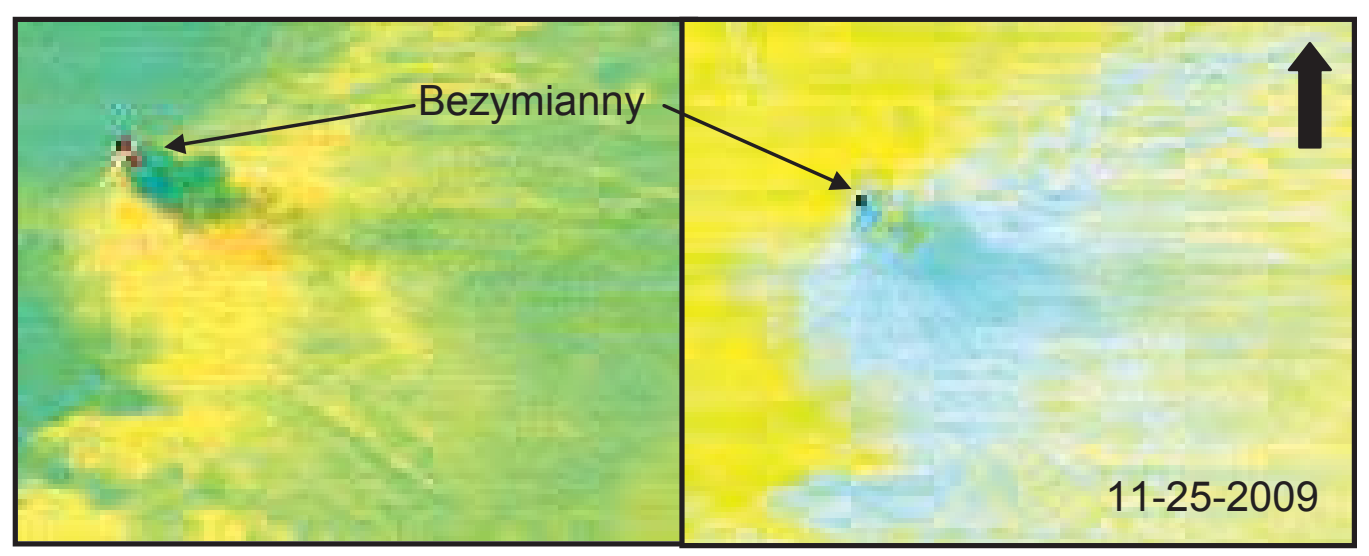

Figure B2: PCA and DCS enhancements of Bezymianny, Russia. 


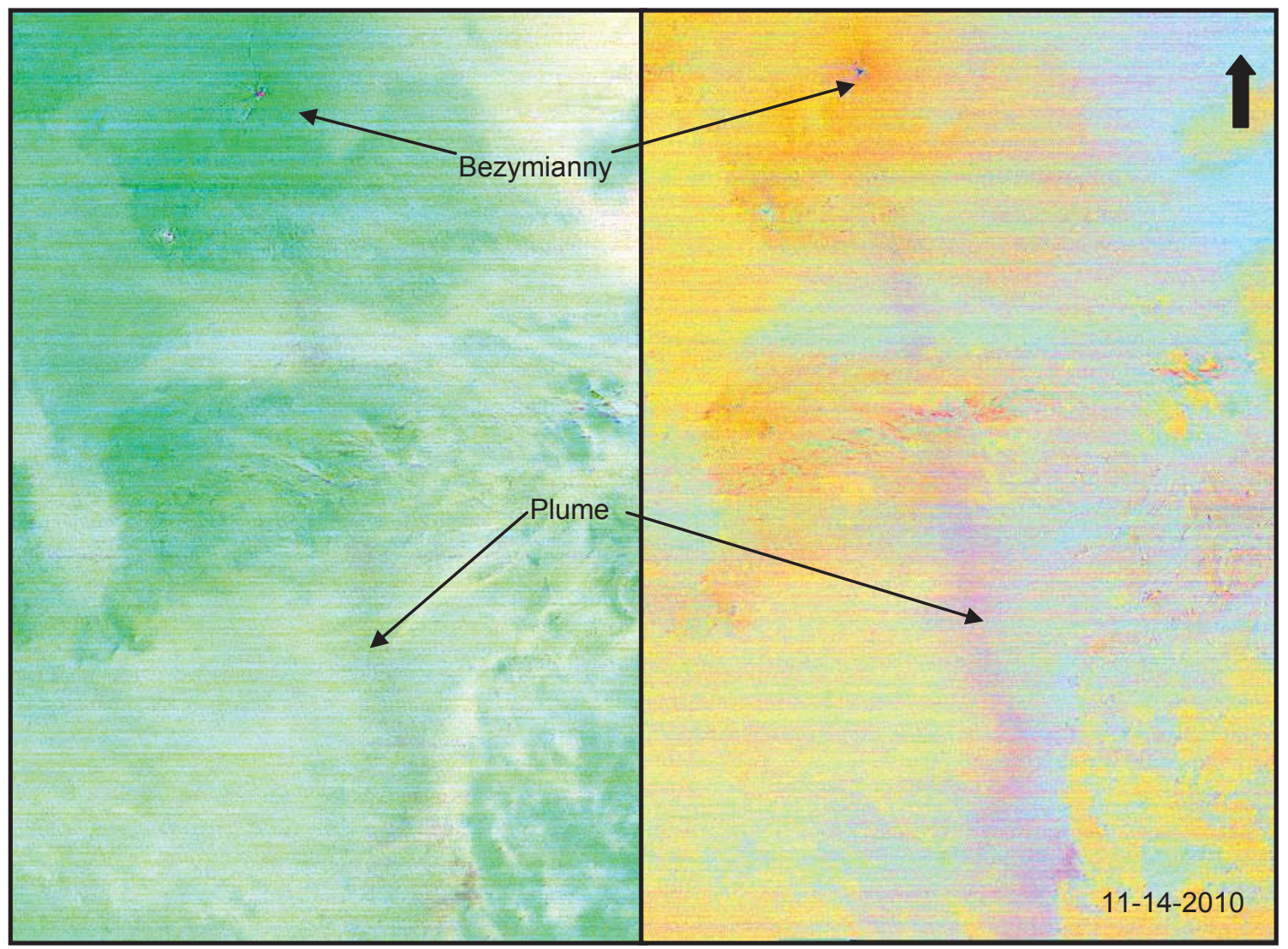

Figure B3: PCA and DCS enhancements of Bezymianny, Russia.

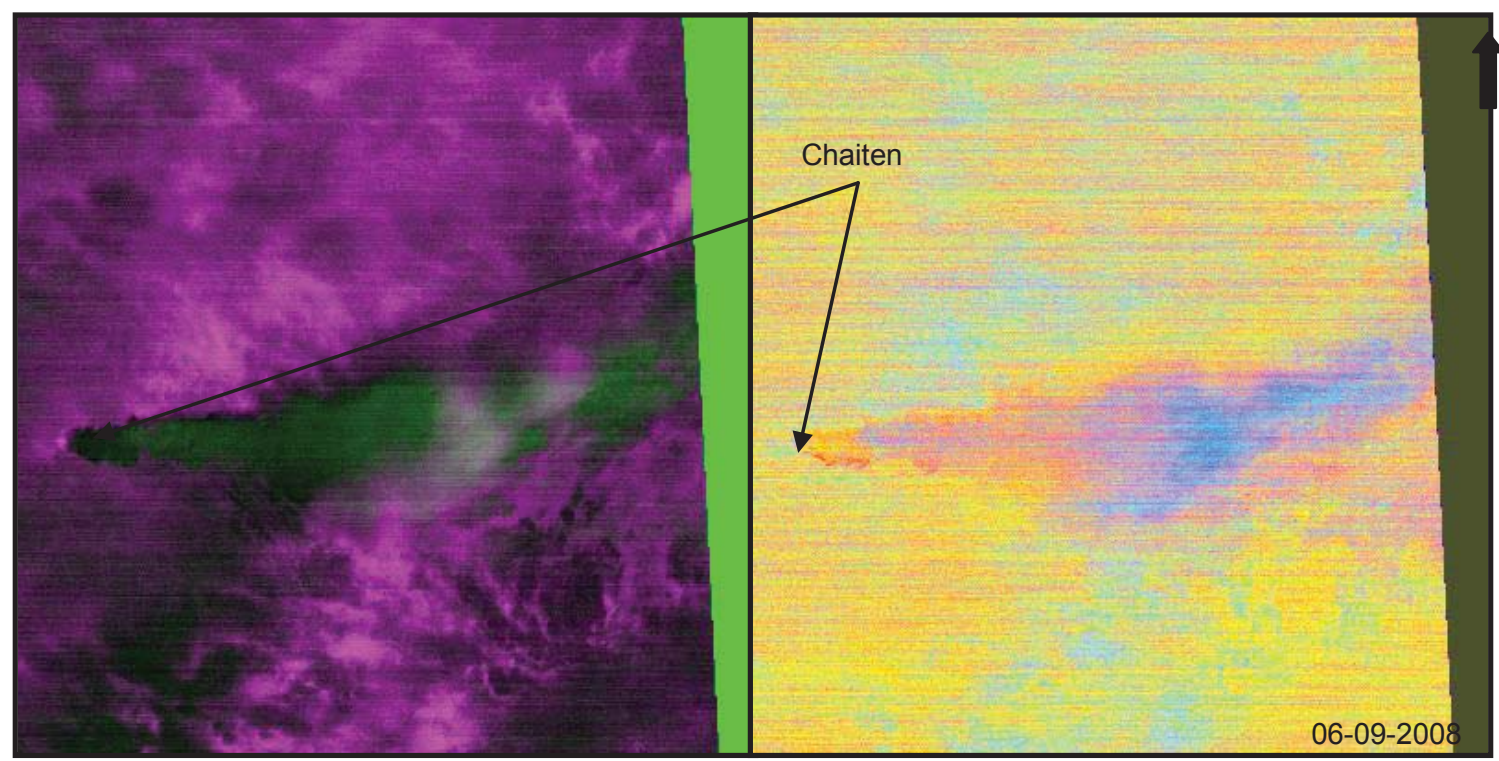

Figure B4: PCA and DCS enhancements of Chaiten, Chile. 

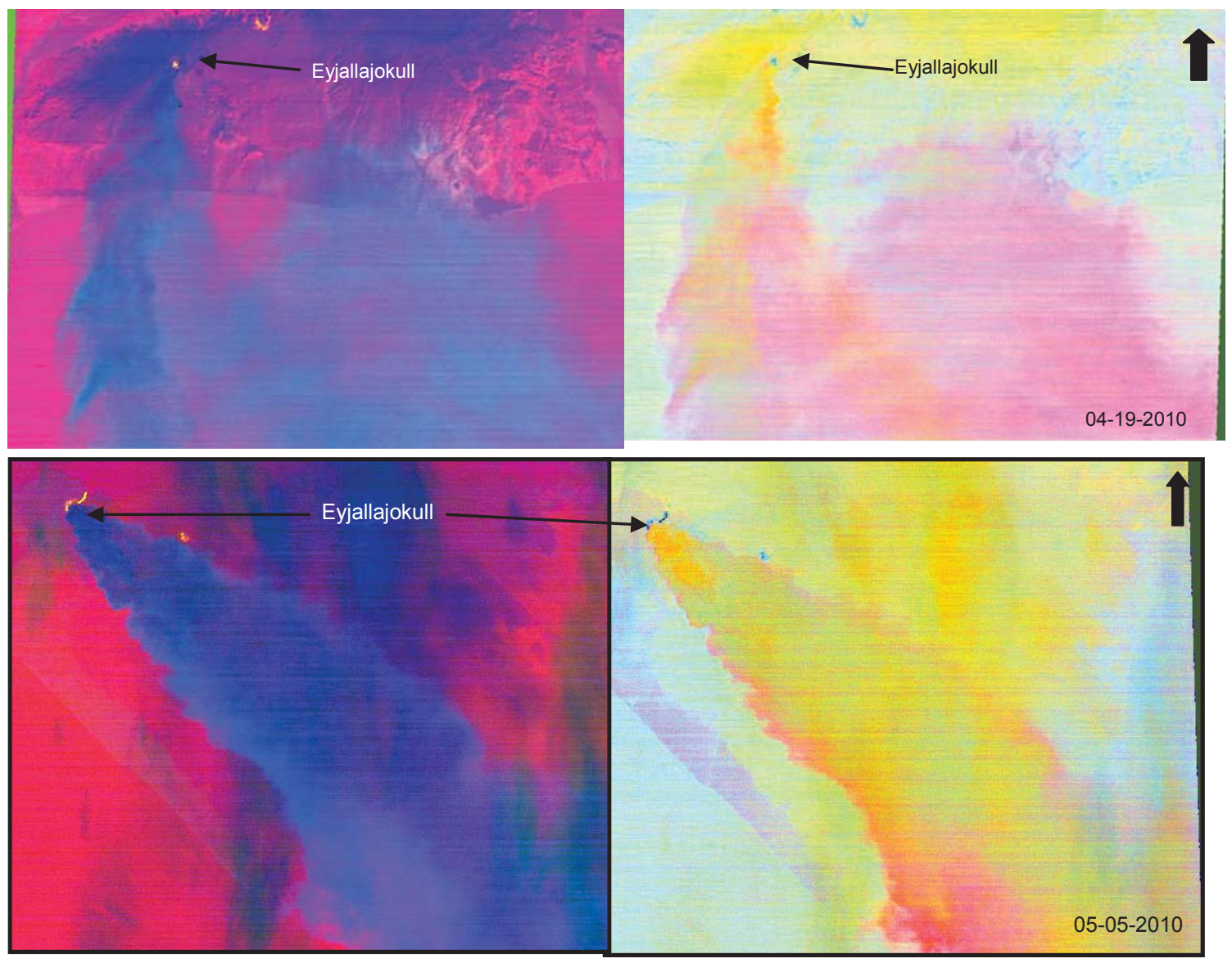

Figure B5: PCA and DCS enhancements of Eyjallajokull, Iceland. 


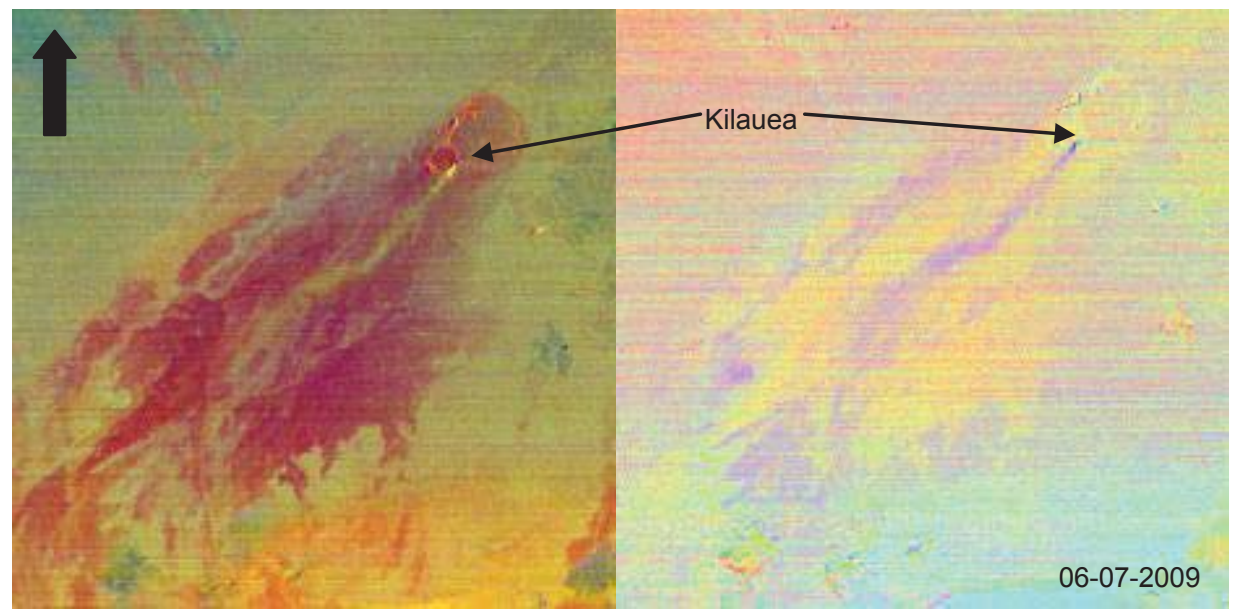

Figure B6: PCA and DCS enhancements of Kilauea, USA.

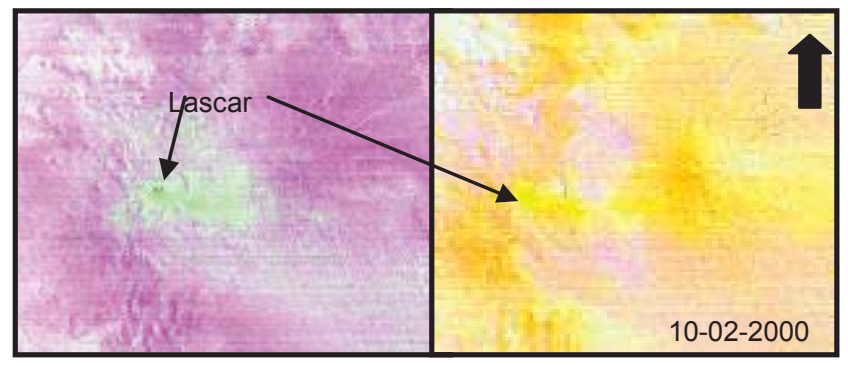

Figure B7: PCA and DCS enhancements of Lascar, Chile. 


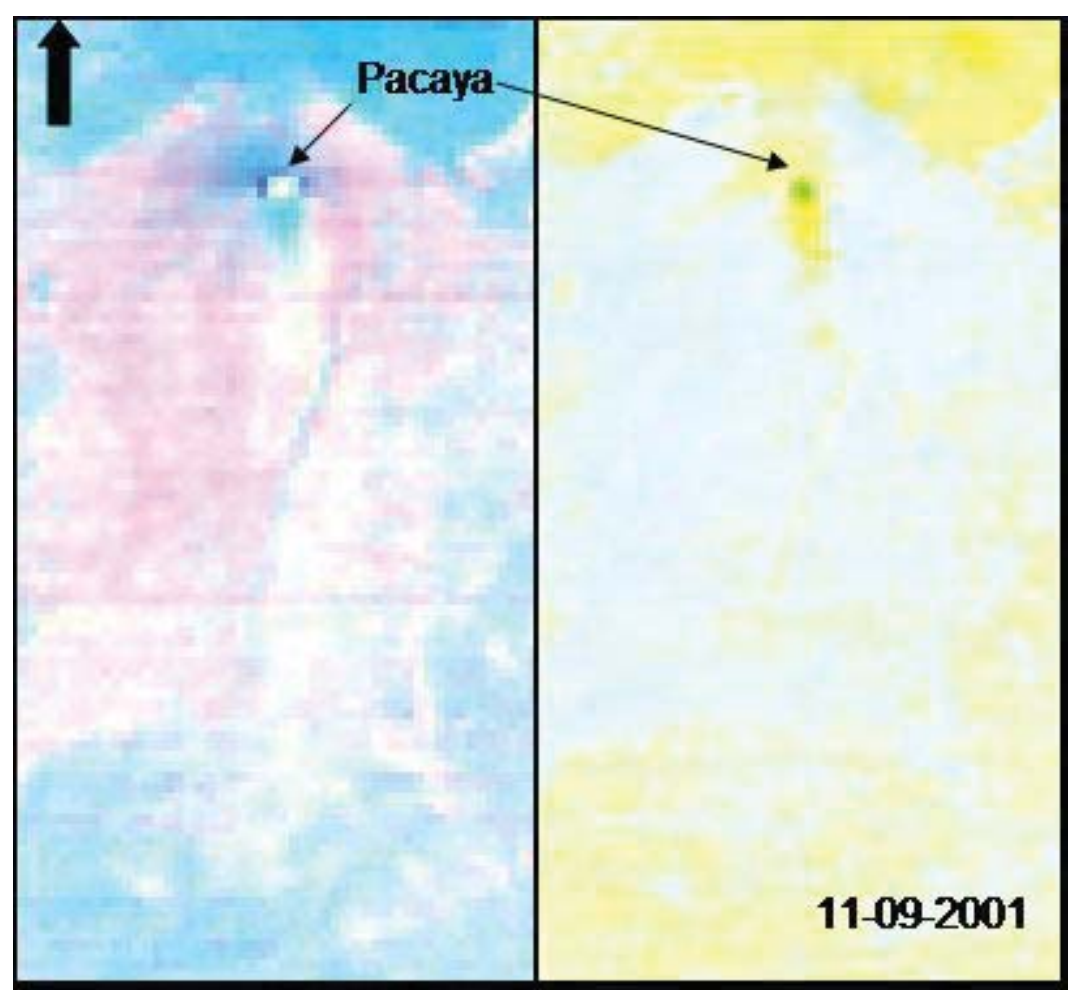

Figure B8: PCA and DCS enhancements of Pacaya, Guatemala.

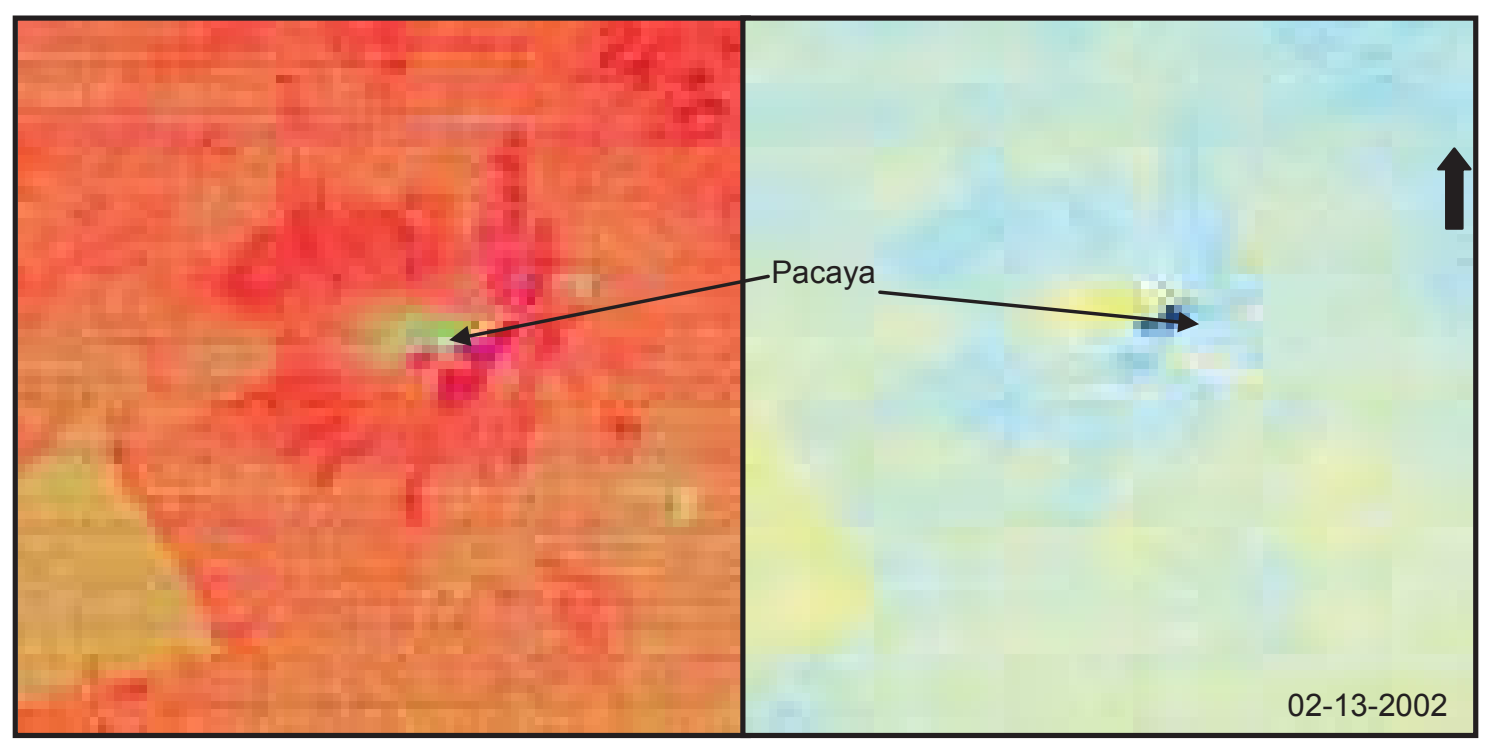

Figure B9: PCA and DCS enhancements of Pacaya, Guatemala. 


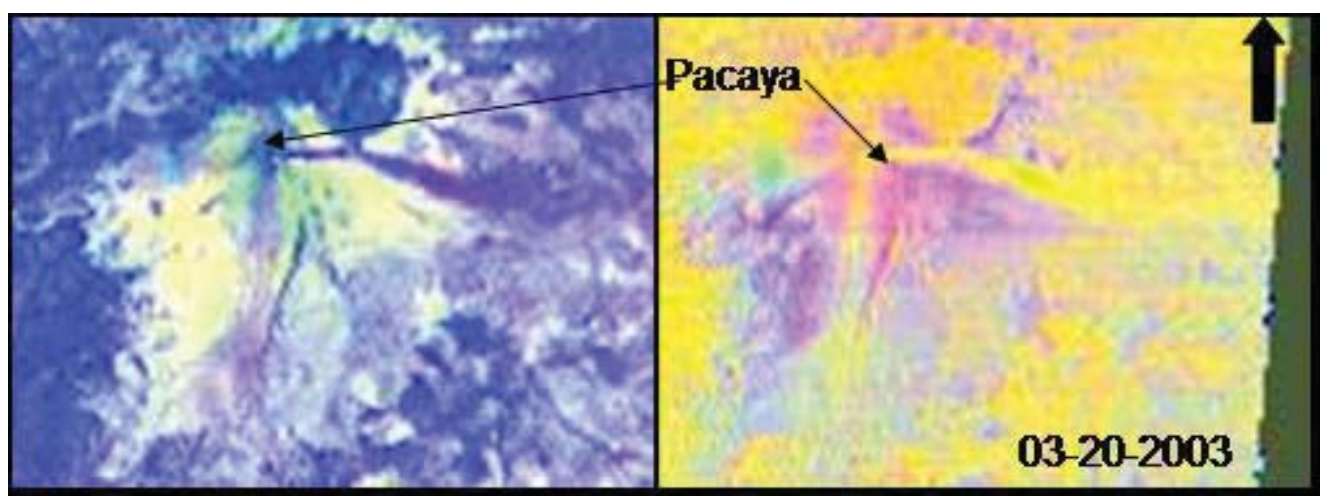

Figure B10: PCA and DCS enhancements of Pacaya, Guatemala. 


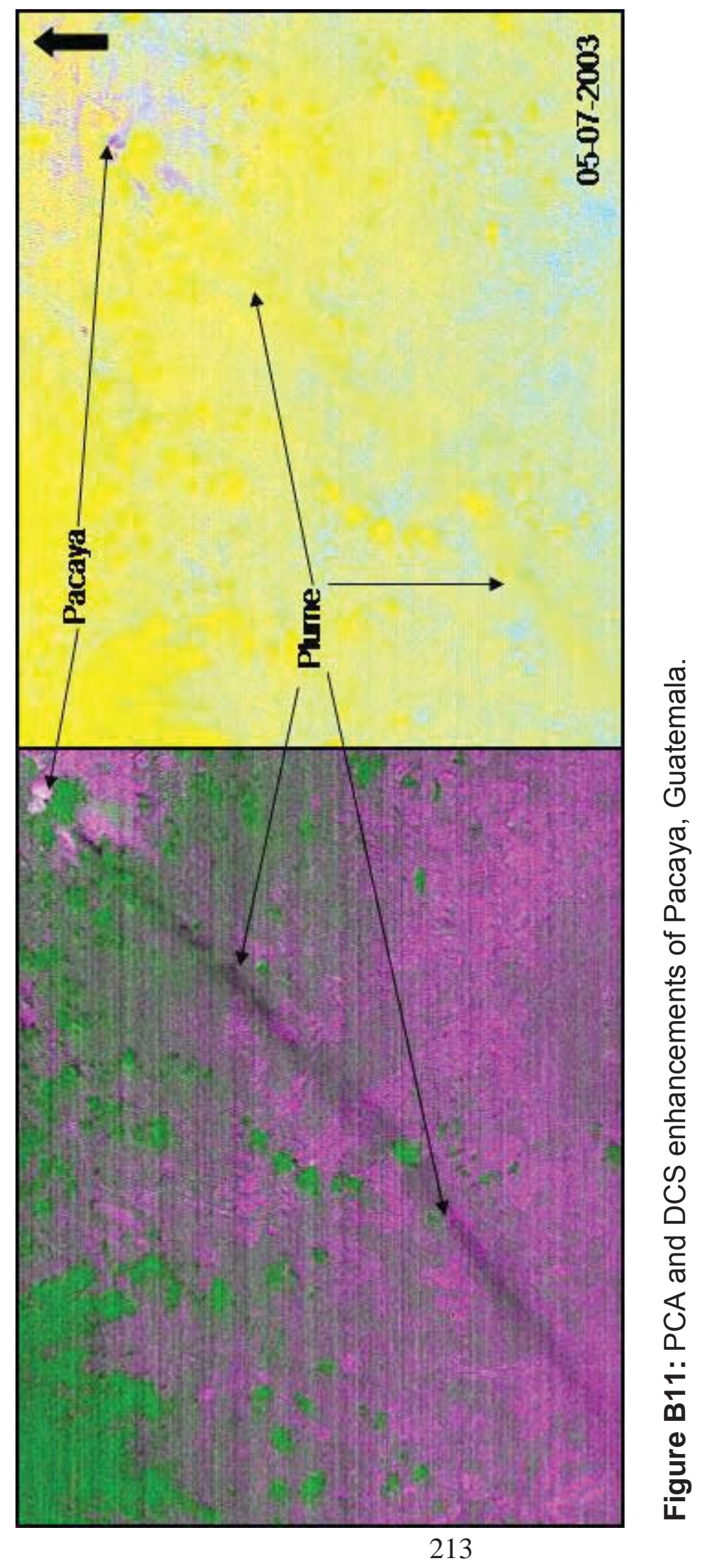




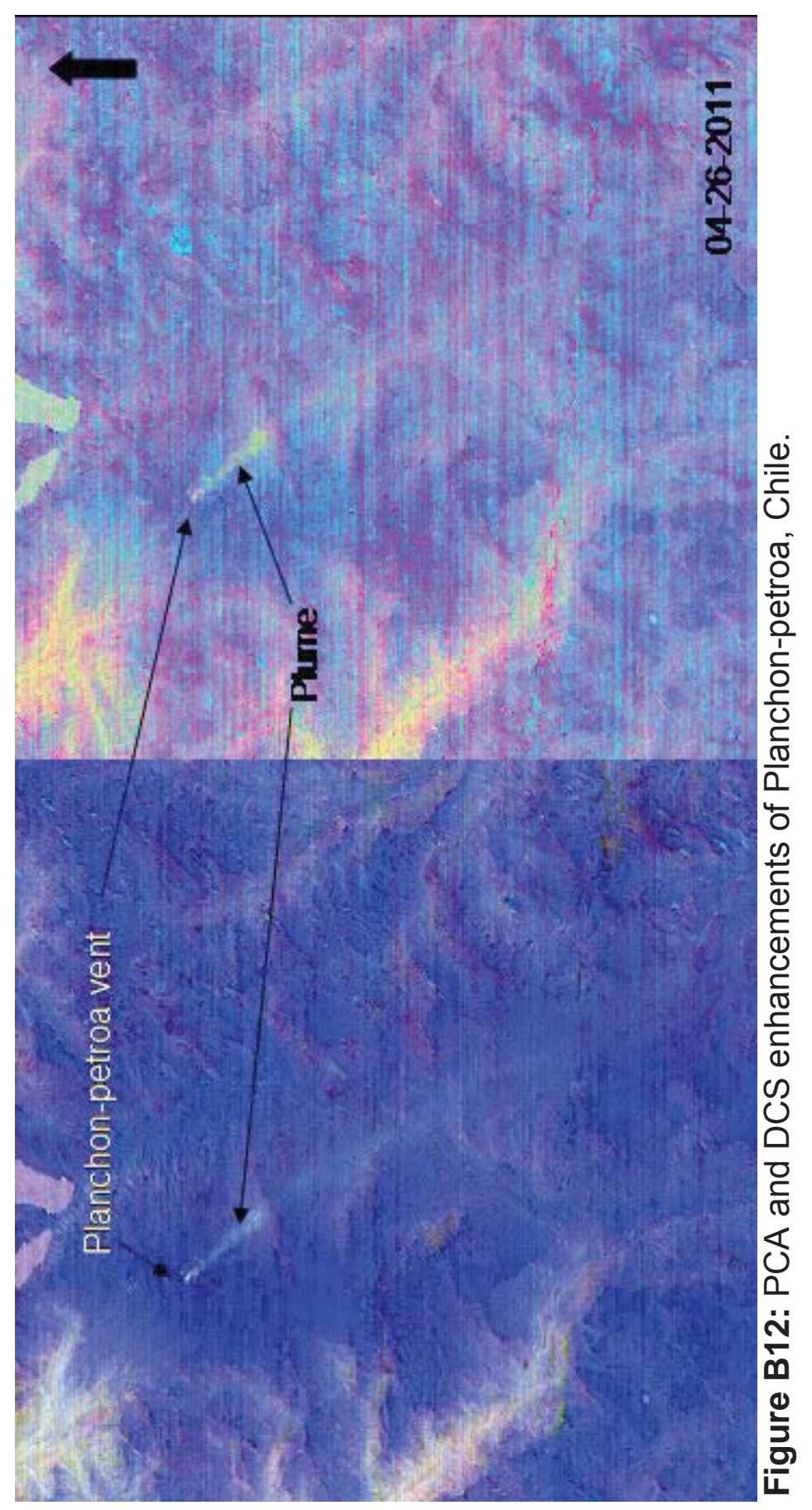




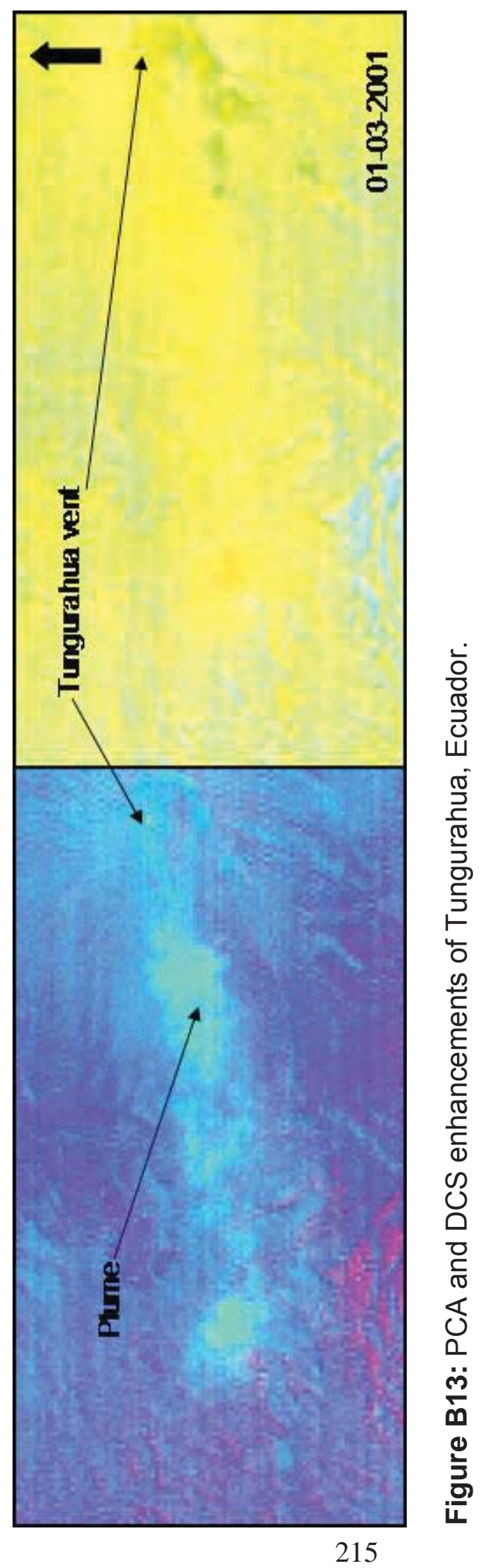




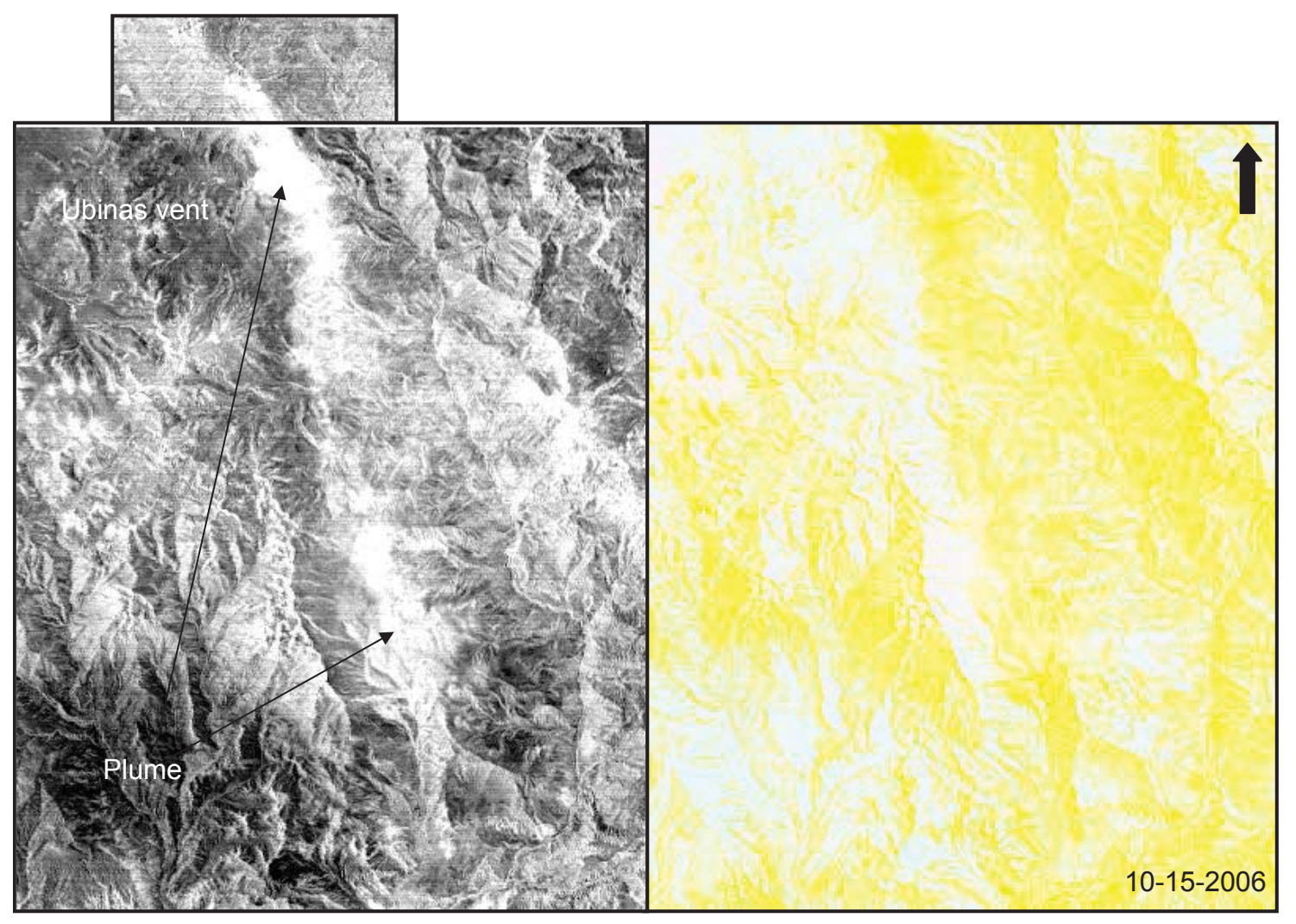

Figure B14: PCA and DCS enhancements of Ubinas, Peru. 


\section{Appendix C}

\section{ASTER, OMI and Ground Based $\mathrm{SO}_{2}$ measurement data points utilized in Chapter 5.}


Table C1

ASTER and Ground based Data.

\begin{tabular}{|c|c|c|c|}
\hline Pacaya & & & \\
\hline Date & $\mathrm{SO}_{2}\left(\mathrm{t} \mathrm{d}^{-1}\right)$ & Instrument & Source \\
\hline $3 / 1 / 2000$ & 1750 & COSPEC & BGVN 2000 \\
\hline $2 / 1 / 2001$ & 951 & COSPEC & BVGN 2001 \\
\hline $2 / 15 / 2001$ & 1740 & COSPEC & BGVN 2001 \\
\hline $2 / 22 / 2001$ & 550 & COSPEC & BGVN 2001 \\
\hline $2 / 28 / 2001$ & 1448 & COSPEC & BGVN 2001 \\
\hline $3 / 8 / 2001$ & 1673 & COSPEC & BGVN 2001 \\
\hline $3 / 29 / 2001$ & 1250 & COSPEC & Rodriguez et al. 2004 \\
\hline $3 / 30 / 2001$ & 1690 & COSPEC & Rodriguez et al. 2004 \\
\hline $4 / 5 / 2001$ & 2380 & COSPEC & Rodriguez et al. 2004 \\
\hline $11 / 9 / 2001$ & 233 & ASTER & This study \\
\hline $11 / 15 / 2001$ & 1950 & COSPEC & Rodriguez et al. 2004 \\
\hline $1 / 6 / 2002$ & 1810 & COSPEC & Rodriguez et al. 2004 \\
\hline $1 / 7 / 2002$ & 660 & COSPEC & Rodriguez et al. 2004 \\
\hline $1 / 12 / 2002$ & 570 & COSPEC & Rodriguez et al. 2004 \\
\hline $1 / 14 / 2002$ & 890 & COSPEC & Rodriguez et al. 2004 \\
\hline $1 / 17 / 2002$ & 2140 & COSPEC & Rodriguez et al. 2004 \\
\hline $1 / 18 / 2002$ & 560 & COSPEC & Rodriguez et al. 2004 \\
\hline $2 / 13 / 2002$ & 2147 & ASTER & This study \\
\hline $9 / 12 / 2002$ & 2200 & COSPEC & Rodriguez et al. 2004 \\
\hline $3 / 1 / 2002$ & 1965 & ASTER & This study \\
\hline $3 / 20 / 2002$ & 1005 & ASTER & This study \\
\hline $7 / 5 / 2003$ & 847 & ASTER & This study \\
\hline \multicolumn{4}{|l|}{ Fuego } \\
\hline Date & $\mathrm{SO}_{2}\left(\mathrm{t} \mathrm{d}^{-1}\right)$ & Instrument & Source \\
\hline $5 / 12 / 2001$ & 140 & COSPEC & Rodriguez et al. 2004 \\
\hline $5 / 25 / 2001$ & 160 & COSPEC & Rodriguez et al. 2004 \\
\hline 9/19/2001 & 140 & COSPEC & Rodriguez et al. 2004 \\
\hline $11 / 11 / 2001$ & 140 & COSPEC & Rodriguez et al. 2004 \\
\hline $11 / 15 / 2001$ & 140 & COSPEC & Rodriguez et al. 2004 \\
\hline $1 / 7 / 2002$ & 223 & COSPEC & Rodriguez et al. 2004 \\
\hline $1 / 14 / 2002$ & 330 & COSPEC & Rodriguez et al. 2004 \\
\hline
\end{tabular}


Table C.1 Continued

\begin{tabular}{|l|r|l|l|}
\hline $2 / 2 / 2002$ & 248.7 & COSPEC & Rodriguez et al. 2004 \\
\hline $2 / 15 / 2002$ & 364.9 & COSPEC & Rodriguez et al. 2004 \\
\hline $2 / 19 / 2002$ & 356.4 & COSPEC & Rodriguez et al. 2004 \\
\hline $3 / 1 / 2002$ & 532 & ASTER & This study \\
\hline $3 / 1 / 2002$ & 512.4 & COSPEC & Rodriguez et al. 2004 \\
\hline $3 / 6 / 2002$ & 652.6 & COSPEC & Rodriguez et al. 2004 \\
\hline $3 / 22 / 2002$ & 823 & COSPEC & Rodriguez et al. 2004 \\
\hline $4 / 17 / 2002$ & 464.9 & COSPEC & Rodriguez et al. 2004 \\
\hline $4 / 19 / 2002$ & 587 & COSPEC & Rodriguez et al. 2004 \\
\hline 6/18/2002 & 318.5 & COSPEC & Rodriguez et al. 2004 \\
\hline $8 / 2 / 2002$ & 394 & COSPEC & Rodriguez et al. 2004 \\
\hline $8 / 20 / 2002$ & 216.1 & COSPEC & Rodriguez et al. 2004 \\
\hline & & & \\
\hline Santiaguito & & & \\
\hline Date & (t d $)$ & Instrument & Source \\
\hline $1 / 25 / 2000$ & 229 & COSPEC & BVGN 2000 \\
\hline $1 / 26 / 2000$ & 90 & COSPEC & BVGN 2000 \\
\hline $1 / 27 / 2000$ & 217.5 & COSPEC & BVGN 2000 \\
\hline $1 / 28 / 2000$ & 193 & COSPEC & BVGN 2000 \\
\hline $1 / 29 / 2000$ & 150 & COSPEC & Rodriguez et al. 2004 \\
\hline $1 / 30 / 2000$ & 90 & COSPEC & Rodriguez et al. 2004 \\
\hline $1 / 31 / 2000$ & 80 & COSPEC & Rodriguez et al. 2004 \\
\hline $2 / 1 / 2000$ & 130 & COSPEC & Rodriguez et al. 2004 \\
\hline $2 / 2 / 2000$ & 160 & COSPEC & Rodriguez et al. 2004 \\
\hline $2 / 28 / 2001$ & 190 & COSPEC & Rodriguez et al. 2004 \\
\hline $9 / 20 / 2001$ & 190 & COSPEC & Rodriguez et al. 2004 \\
\hline $1 / 9 / 2002$ & 20 & COSPEC & Rodriguez et al. 2004 \\
\hline $1 / 10 / 2002$ & 170 & COSPEC & Rodriguez et al. 2004 \\
\hline $1 / 11 / 2002$ & 40 & COSPEC & Rodriguez et al. 2004 \\
\hline $4 / 13 / 2002$ & 120 & COSPEC & Rodriguez et al. 2004 \\
\hline $4 / 17 / 2002$ & 180 & COSPEC & Rodriguez et al. 2004 \\
\hline $1 / 29 / 2008$ & 92 & Camera & Holland et al. 2011 \\
\hline $2 / 2 / 2008$ & 76.3 & Camera & Holland et al. 2011 \\
\hline $2 / 7 / 2009$ & 47 & Camera & Holland et al. 2011 \\
\hline $2 / 9 / 2009$ & 60.5 & Camera & Holland et al. 2011 \\
\hline $2 / 12 / 2009$ & 69 & Camera & Holland et al. 2011 \\
\hline & & & \\
\hline Tacana & & & \\
\hline & & & \\
\hline & SO & & \\
\hline & & & \\
\hline & & &
\end{tabular}


Table C.1 Continued

\begin{tabular}{|c|c|c|c|}
\hline Date & $\mathrm{SO}_{2}\left(\mathrm{t} \mathrm{d}^{-1}\right)$ & Instrument & Source \\
\hline 11/14/2011 & 30 & COSPEC & Rodriguez et al. 2004 \\
\hline \multicolumn{4}{|l|}{ San Miguel } \\
\hline Date & $\mathrm{SO}_{2}\left(\mathrm{t} \mathrm{d}^{-1}\right)$ & Instrument & Source \\
\hline $1 / 28 / 2002$ & 280 & COSPEC & Rodriguez et al. 2004 \\
\hline $1 / 28 / 2002$ & 280 & COSPEC & Rodriguez et al. 2004 \\
\hline \multicolumn{4}{|l|}{ Santa Ana } \\
\hline Date & $\mathrm{SO}_{2}\left(\mathrm{t} \mathrm{d}^{-1}\right)$ & Instrument & Source \\
\hline 2/8/2001 & 393 & COSPEC & BVGN 2001 \\
\hline 2/8/2001 & 110 & COSPEC & Rodriguez et al. 2004 \\
\hline 2/9/2001 & 244 & COSPEC & BVGN 2001 \\
\hline $5 / 9 / 2001$ & 280 & COSPEC & Rodriguez et al. 2004 \\
\hline $5 / 9 / 2001$ & 170 & COSPEC & Rodriguez et al. 2004 \\
\hline $1 / 24 / 2002$ & 80 & COSPEC & Rodriguez et al. 2004 \\
\hline $1 / 25 / 2002$ & 30 & COSPEC & Rodriguez et al. 2004 \\
\hline $1 / 26 / 2002$ & 50 & COSPEC & Rodriguez et al. 2004 \\
\hline $9 / 16 / 2005$ & 3320 & COSPEC & BVGN 2005 \\
\hline \multirow[t]{2}{*}{ 9/18/2005 } & 1472 & COSPEC & Barrancos et al. 2008 \\
\hline & 1406 & DOAS & Barrancos et al. 2008 \\
\hline \multirow[t]{2}{*}{ 9/20/2005 } & 1626 & COSPEC & Barrancos et al. 2008 \\
\hline & 1786 & DOAS & Barrancos et al. 2008 \\
\hline $9 / 21 / 2005$ & 3558 & DOAS & Barrancos et al. 2008 \\
\hline \multirow[t]{2}{*}{ 9/22/2005 } & 4624 & COSPEC & Barrancos et al. 2008 \\
\hline & 4481 & DOAS & Barrancos et al. 2008 \\
\hline \multirow[t]{2}{*}{ 9/24/2005 } & 2151 & COSPEC & Barrancos et al. 2008 \\
\hline & 2418 & DOAS & Barrancos et al. 2008 \\
\hline \multirow[t]{2}{*}{ 9/25/2005 } & 3136 & COSPEC & Barrancos et al. 2008 \\
\hline & 3344 & DOAS & Barrancos et al. 2008 \\
\hline \multirow[t]{2}{*}{ 9/26/2005 } & 3136 & DOAS & Barrancos et al. 2008 \\
\hline & 3344 & COSPEC & Barrancos et al. 2008 \\
\hline \multirow[t]{2}{*}{ 9/27/2005 } & 736 & DOAS & Barrancos et al. 2008 \\
\hline & 768 & COSPEC & Barrancos et al. 2008 \\
\hline $10 / 11 / 2005$ & 650 & COSPEC & BVGN 2005 \\
\hline $2 / 20 / 2006$ & 297 & DOAS & Barrancos et al.2008 \\
\hline \multirow[t]{2}{*}{ 2/21/2006 } & 679 & COSPEC & Barrancos et al. 2008 \\
\hline & 579 & DOAS & Barrancos et al. 2008 \\
\hline
\end{tabular}


Table C.1 Continued

\begin{tabular}{|c|c|c|c|}
\hline $2 / 22 / 2006$ & 787 & DOAS & Barrancos et al. 2008 \\
\hline \multirow[t]{2}{*}{ 2/23/2006 } & 414 & COSPEC & Barrancos et al. 2008 \\
\hline & 396 & DOAS & Barrancos et al. 2008 \\
\hline $2 / 24 / 2006$ & 565 & DOAS & Barrancos et al. 2008 \\
\hline \multirow[t]{2}{*}{ 2/26/2006 } & 400 & COSPEC & Barrancos et al. 2008 \\
\hline & 391 & DOA & Barrancos et al. 2008 \\
\hline \multirow[t]{2}{*}{ 2/27/2006 } & 396 & COSPEC & Barrancos et al. 2008 \\
\hline & 418 & DOAS & Barrancos et al. 2008 \\
\hline \multirow[t]{2}{*}{ 2/28/2008 } & 354 & COSPEC & Barrancos et al. 2008 \\
\hline & 365 & DOAS & Barrancos et al. 2008 \\
\hline \multicolumn{4}{|l|}{ Concepcion } \\
\hline Date & $\mathrm{SO}_{2}\left(\mathrm{t} \mathrm{d}^{-1}\right)$ & Instrument & Source \\
\hline $8 / 19 / 2005$ & 400 & COSPEC & BVGN2005 \\
\hline \multicolumn{4}{|l|}{ Telica } \\
\hline Date & $\mathrm{SO}_{2}\left(\mathrm{t} \mathrm{d}^{-1}\right)$ & Instrument & Source \\
\hline November 2003 & 530 & DOAS & Mather et al. 2006 \\
\hline \multicolumn{4}{|l|}{ San Cristobal } \\
\hline Date & $\mathrm{SO}_{2}\left(\mathrm{t} \mathrm{d}^{-1}\right)$ & Instrument & Source \\
\hline $3 / 19 / 2000$ & 352 & COSPEC & BVGN 2005 \\
\hline $3 / 20 / 2000$ & 1644 & COSPEC & BVGN 2005 \\
\hline $1 / 30 / 2002$ & 3070 & ASTER & This study \\
\hline $11 / 30 / 2003$ & 800 & DOAS & Mather et al. 2006 \\
\hline $1 / 29 / 2004$ & 2704 & ASTER & This study \\
\hline $11 / 19 / 2004$ & 1364 & ASTER & This study \\
\hline $2 / 7 / 2005$ & 3444 & ASTER & This study \\
\hline 3/7/2006 & 5247 & DOAS & Barrancos et al. 2008 \\
\hline $3 / 11 / 2006$ & 317 & DOAS & Barrancos et al. 2008 \\
\hline $3 / 12 / 2006$ & 224 & DOAS & Barrancos et al. 2008 \\
\hline $1 / 15 / 2009$ & 1915 & ASTER & This study \\
\hline $1 / 31 / 2010$ & 352 & ASTER & This study \\
\hline \multicolumn{4}{|l|}{ Masaya } \\
\hline Date & $\mathrm{SO}_{2}\left(\mathrm{t} \mathrm{d}^{-1}\right)$ & Instrument & Source \\
\hline $11 / 18 / 2003$ & 173 & DOAS & Mather et al. 2006 \\
\hline $11 / 25 / 2003$ & 293 & DOAS & Mather et al. 2006 \\
\hline
\end{tabular}


Table C.1 Continued

\begin{tabular}{|c|c|c|c|}
\hline $12 / 1 / 2003$ & 180 & DOAS & Mather et al. 2006 \\
\hline 2/9/2005 & 1362 & ASTER & This study \\
\hline \multirow{2}{*}{$2 / 25 / 2005$} & 1684 & Flyspec & Nadeau et al. 2009 \\
\hline & 2098 & & Nadeau et al. 2009 \\
\hline \multirow[t]{2}{*}{$2 / 26 / 2005$} & 3153 & Flyspec & Nadeau et al. 2009 \\
\hline & 2688 & & Nadeau et al. 2009 \\
\hline $3 / 3 / 2005$ & 339 & Flyspec & Nadeau et al. 2009 \\
\hline 3/5/2005 & 182 & Flyspec & Nadeau et al. 2009 \\
\hline $3 / 6 / 2005$ & 136 & Flyspec & Nadeau et al. 2009 \\
\hline \multirow{2}{*}{ 3/7/2005 } & 811 & Flyspec & Nadeau et al. 2009 \\
\hline & 636 & & Nadeau et al. 2009 \\
\hline $3 / 11 / 2005$ & 137 & Flyspec & Nadeau et al. 2009 \\
\hline \multirow[t]{2}{*}{$3 / 12 / 2005$} & 249 & Flyspec & Nadeau et al. 2009 \\
\hline & 161 & & Nadeau et al. 2009 \\
\hline \multirow[t]{2}{*}{ 3/14/2005 } & 254 & Flyspec & Nadeau et al. 2009 \\
\hline & 128 & & Nadeau et al. 2009 \\
\hline \multirow[t]{2}{*}{$3 / 15 / 2005$} & 965 & Flyspec & Nadeau et al. 2009 \\
\hline & 663 & & \\
\hline 3/16/2005 & 1217 & Flyspec & Nadeau et al. 2009 \\
\hline \multirow[t]{2}{*}{$2 / 16 / 2006$} & 646 & Flyspec & Nadeau et al. 2009 \\
\hline & 436 & & Nadeau et al. 2009 \\
\hline \multirow[t]{2}{*}{$2 / 17 / 2006$} & 340 & Flyspec & Nadeau et al. 2009 \\
\hline & 445 & & Nadeau et al. 2009 \\
\hline \multirow[t]{2}{*}{$2 / 18 / 2006$} & 189 & Flyspec & Nadeau et al. 2009 \\
\hline & 346 & & Nadeau et al. 2009 \\
\hline \multirow[t]{2}{*}{$2 / 19 / 2006$} & 115 & Flyspec & Nadeau et al. 2009 \\
\hline & 446 & & Nadeau et al. 2009 \\
\hline \multirow[t]{2}{*}{$2 / 20 / 2006$} & 211 & Flyspec & Nadeau et al. 2009 \\
\hline & 342 & & Nadeau et al. 2009 \\
\hline \multirow[t]{2}{*}{$2 / 21 / 2006$} & 115 & Flyspec & Nadeau et al. 2009 \\
\hline & 264 & & Nadeau et al. 2009 \\
\hline 2/22/2006 & 1700 & Filter pack & Witt et al. 2008 \\
\hline \multirow[t]{2}{*}{$2 / 23 / 2006$} & 1983 & Flyspec & Nadeau et al. 2009 \\
\hline & 1035 & & Nadeau et al. 2009 \\
\hline \multirow[t]{2}{*}{$2 / 24 / 2006$} & 311 & Flyspec & Nadeau et al. 2009 \\
\hline & 676 & & Nadeau et al. 2009 \\
\hline $2 / 25 / 2006$ & 1500 & Filter pack & Witt et al. 2008 \\
\hline 2/26/2005 & 3552 & Flyspec & Nadeau et al. 2009 \\
\hline
\end{tabular}




\section{Table C.1 Continued}

\begin{tabular}{|c|c|c|c|}
\hline & 2228 & & Nadeau et al. 2009 \\
\hline \multirow[t]{2}{*}{$2 / 27 / 2006$} & 1212 & Flyspec & Nadeau et al. 2009 \\
\hline & 778 & & Nadeau et al. 2009 \\
\hline \multirow[t]{2}{*}{$2 / 28 / 2006$} & 2876 & Flyspec & Nadeau et al. 2009 \\
\hline & 1144 & & Nadeau et al. 2009 \\
\hline \multirow[t]{2}{*}{$3 / 1 / 2006$} & 3224 & Flyspec & Nadeau et al. 2009 \\
\hline & 1524 & & Nadeau et al. 2009 \\
\hline \multirow[t]{3}{*}{$3 / 2 / 2005$} & 1321 & Flyspec & Nadeau et al. 2009 \\
\hline & 749 & & Nadeau et al. 2009 \\
\hline & 1130 & Filter Pack & Witt et al. 2008 \\
\hline \multirow[t]{2}{*}{$3 / 3 / 2006$} & 1127 & Flyspec & Nadeau et al. 2009 \\
\hline & 727 & & Nadeau et al. 2009 \\
\hline \multirow[t]{3}{*}{$3 / 4 / 3006$} & 943 & Flyspec & Nadeau et al. 2009 \\
\hline & 622 & & Nadeau et al. 2009 \\
\hline & 650 & Filter Pack & Witt et al. 2008 \\
\hline \multirow[t]{2}{*}{$3 / 6 / 2006$} & 989 & Flyspec & Nadeau et al. 2009 \\
\hline & 523 & & Nadeau et al. 2009 \\
\hline \multirow[t]{2}{*}{$3 / 7 / 2006$} & 837 & Flyspec & Nadeau et al. 2009 \\
\hline & 466 & & Nadeau et al. 2009 \\
\hline \multirow[t]{2}{*}{$3 / 8 / 2006$} & 528 & Flyspec & Nadeau et al. 2009 \\
\hline & 283 & & Nadeau et al. 2009 \\
\hline \multirow[t]{2}{*}{$3 / 12 / 2006$} & 3014 & Flyspec & Nadeau et al. 2009 \\
\hline & 1581 & & Nadeau et al. 2009 \\
\hline \multirow[t]{2}{*}{$3 / 13 / 2006$} & 1183 & COSPEC & Barrancos et al. 2008 \\
\hline & 965 & & Barrancos et al. 2008 \\
\hline \multirow[t]{2}{*}{$3 / 14 / 2006$} & 842 & COSPEC & Barrancos et al. 2008 \\
\hline & 819 & & Barrancos et al. 2008 \\
\hline $3 / 15 / 2006$ & 1018 & COSPEC & Barrancos et al. 2008 \\
\hline \multirow[t]{2}{*}{$3 / 16 / 2006$} & 1031 & COSPEC & Barrancos et al. 2008 \\
\hline & 1098 & & Barrancos et al. 2008 \\
\hline $12 / 6 / 2006$ & 1458 & COSPEC & Barrancos et al. 2008 \\
\hline $12 / 8 / 2006$ & 990 & DOAS & Barrancos et al. 2008 \\
\hline $12 / 10 / 2006$ & 1046 & DOAS & Barrancos et al. 2008 \\
\hline \multirow[t]{2}{*}{$3 / 8 / 2007$} & 771 & Flyspec & Nadeau et al. 2009 \\
\hline & 861 & & Nadeau et al. 2009 \\
\hline \multicolumn{4}{|l|}{ POAS } \\
\hline Date & $\mathrm{SO}_{2}\left(\mathrm{t} \mathrm{d}^{-1}\right)$ & Instrument & Source \\
\hline
\end{tabular}


Table C.1 Continued

\begin{tabular}{|c|c|c|c|}
\hline March 2001 & 8 & COSPEC & Zimmer et al. 2004 \\
\hline $4 / 21 / 2006$ & 99 & DOAS & Barrancos et al. 2008 \\
\hline $4 / 24 / 2006$ & 160 & DOAS & Barrancos et al. 2008 \\
\hline $4 / 27 / 2006$ & 86 & DOAS & Barrancos et al. 2008 \\
\hline $4 / 30 / 2006$ & 90 & DOAS & Barrancos et al. 2008 \\
\hline \multicolumn{4}{|l|}{ Arenal } \\
\hline Date & $\mathrm{SO}_{2}\left(\mathrm{t} \mathrm{d}^{-1}\right)$ & Instrument & Source \\
\hline $3 / 1 / 2001$ & 180 & COSPEC & Zimmer et al. 2004 \\
\hline $8 / 29 / 2005$ & 550 & ASTER & This study \\
\hline \multirow[t]{2}{*}{$4 / 25 / 2006$} & 154 & COSPEC & Barrancos et al. 2008 \\
\hline & 121 & DOAS & Barrancos et al. 2008 \\
\hline $4 / 26 / 2006$ & 106 & DOAS & Barrancos et al. 2008 \\
\hline $4 / 6 / 2008$ & 184 & ASTER & This study \\
\hline \multicolumn{4}{|l|}{ Turrialba } \\
\hline 09/09/2009 & 219 & ASTER & This study \\
\hline $1 / 31 / 2010$ & 529 & ASTER & This study \\
\hline $1 / 31 / 2010$ & 763 & ASTER & This study \\
\hline $2 / 16 / 2010$ & 4236 & ASTER & This study \\
\hline $4 / 5 / 2010$ & 1166 & ASTER & This study \\
\hline $6 / 24 / 2010$ & 4047 & ASTER & This study \\
\hline $12 / 17 / 2010$ & 3596 & ASTER & This study \\
\hline $1 / 18 / 2011$ & 2153 & ASTER & This study \\
\hline \multicolumn{4}{|l|}{ Galeras } \\
\hline Date & $\mathrm{SO}_{2}\left(\mathrm{t} \mathrm{d}^{-1}\right)$ & Instrument & Source \\
\hline $12 / 19 / 2005$ & 783 & & INGEOMINAS \\
\hline $12 / 20 / 2005$ & 590 & & INGEOMINAS \\
\hline $1 / 9 / 2006$ & 300 & & INGEOMINAS \\
\hline $1 / 16 / 2006$ & 710 & & INGEOMINAS \\
\hline $1 / 23 / 2006$ & 200 & & INGEOMINAS \\
\hline $1 / 30 / 2006$ & 600 & & INGEOMINAS \\
\hline $2 / 6 / 2006$ & 800 & & INGEOMINAS \\
\hline $2 / 13 / 2006$ & 700 & & INGEOMINAS \\
\hline $2 / 20 / 2006$ & 120 & & INGEOMINAS \\
\hline $2 / 27.2006$ & 685 & & INGEOMINAS \\
\hline $3 / 28 / 2006$ & 326 & & INGEOMINAS \\
\hline
\end{tabular}


Table C.1 Continued

\begin{tabular}{|c|c|c|}
\hline July 2006 & 1383 & INGEOMINAS \\
\hline $10 / 2 / 2006$ & 1358 & INGEOMINAS \\
\hline $8 / 6 / 2007$ & 1615 & INGEOMINAS \\
\hline $8 / 7 / 2007$ & 760 & INGEOMINAS \\
\hline $8 / 14 / 2007$ & 780 & INGEOMINAS \\
\hline $8 / 31 / 2007$ & 1368 & INGEOMINAS \\
\hline $9 / 10 / 2007$ & 6059 & INGEOMINAS \\
\hline $9 / 17 / 2007$ & 5617 & INGEOMINAS \\
\hline $9 / 24 / 2007$ & 5212 & INGEOMINAS \\
\hline 2/8/2008 & 144400 & INGEOMINAS \\
\hline $6 / 12 / 2008$ & 8541 & INGEOMINAS \\
\hline $6 / 17 / 2008$ & 8560 & INGEOMINAS \\
\hline $7 / 1 / 2008$ & 10800 & INGEOMINAS \\
\hline $7 / 21 / 2008$ & 3600 & INGEOMINAS \\
\hline $7 / 22 / 2008$ & 7800 & INGEOMINAS \\
\hline $7 / 29 / 2008$ & 1506 & INGEOMINAS \\
\hline $8 / 1 / 2008$ & 5600 & INGEOMINAS \\
\hline $8 / 4 / 2008$ & 10000 & INGEOMINAS \\
\hline $8 / 7 / 2008$ & 5200 & INGEOMINAS \\
\hline $8 / 14 / 2008$ & 6000 & INGEOMINAS \\
\hline $8 / 26 / 2008$ & 6200 & INGEOMINAS \\
\hline $8 / 30 / 2008$ & 550 & INGEOMINAS \\
\hline 9/5/2008 & 14500 & INGEOMINAS \\
\hline $9 / 10 / 2008$ & 8200 & INGEOMINAS \\
\hline $9 / 19 / 2008$ & 5200 & INGEOMINAS \\
\hline 9/20/2008 & 6900 & INGEOMINAS \\
\hline $9 / 22 / 2008$ & 3640 & INGEOMINAS \\
\hline $11 / 10 / 2008$ & 4400 & INGEOMINAS \\
\hline $11 / 12 / 2008$ & 2600 & INGEOMINAS \\
\hline $11 / 23 / 2008$ & 700 & INGEOMINAS \\
\hline $11 / 29 / 2008$ & 2900 & INGEOMINAS \\
\hline $12 / 8 / 2008$ & 4600 & INGEOMINAS \\
\hline $12 / 10 / 2008$ & 1100 & INGEOMINAS \\
\hline $12 / 11 / 2008$ & 200 & INGEOMINAS \\
\hline $12 / 23 / 2008$ & 340 & INGEOMINAS \\
\hline $12 / 20 / 2008$ & 1285 & INGEOMINAS \\
\hline $1 / 6 / 2009$ & 240 & INGEOMINAS \\
\hline $1 / 13 / 2009$ & 1780 & INGEOMINAS \\
\hline
\end{tabular}


Table C.1 Continued

\begin{tabular}{|c|c|c|}
\hline $1 / 14 / 2009$ & 1200 & INGEOMINAS \\
\hline $1 / 21 / 2009$ & 200 & INGEOMINAS \\
\hline $1 / 27 / 2009$ & 3300 & INGEOMINAS \\
\hline $1 / 31 / 2009$ & 1800 & INGEOMINAS \\
\hline $2 / 15 / 2009$ & 300 & INGEOMINAS \\
\hline $2 / 16 / 2009$ & 200 & INGEOMINAS \\
\hline $2 / 17 / 2009$ & 500 & INGEOMINAS \\
\hline $2 / 21 / 2009$ & 800 & INGEOMINAS \\
\hline 2/23/2009 & 430 & INGEOMINAS \\
\hline 2/24/2009 & 430 & INGEOMINAS \\
\hline $3 / 3 / 2009$ & 3200 & INGEOMINAS \\
\hline 3/9/2009 & 5650 & INGEOMINAS \\
\hline $3 / 14 / 2009$ & 974 & INGEOMINAS \\
\hline $3 / 15 / 2009$ & 2000 & INGEOMINAS \\
\hline $3 / 16 / 2009$ & 2600 & INGEOMINAS \\
\hline $3 / 17 / 2009$ & 570 & INGEOMINAS \\
\hline $3 / 24 / 2009$ & 1900 & INGEOMINAS \\
\hline $3 / 31 / 2009$ & 400 & INGEOMINAS \\
\hline $4 / 7 / 2009$ & 250 & INGEOMINAS \\
\hline 4/8/2009 & 600 & INGEOMINAS \\
\hline 4/14/2009 & 1400 & INGEOMINAS \\
\hline $4 / 21 / 2009$ & 1300 & INGEOMINAS \\
\hline $5 / 4 / 2009$ & 295 & INGEOMINAS \\
\hline $5 / 5 / 2009$ & 1070 & INGEOMINAS \\
\hline $5 / 7 / 2009$ & 1000 & INGEOMINAS \\
\hline $5 / 12 / 2009$ & 75 & INGEOMINAS \\
\hline $5 / 17 / 2009$ & 530 & INGEOMINAS \\
\hline $5 / 21 / 2009$ & 360 & INGEOMINAS \\
\hline $6 / 1 / 2009$ & 987 & INGEOMINAS \\
\hline 6/8/2009 & 400 & INGEOMINAS \\
\hline 6/19/2009 & 1600 & INGEOMINAS \\
\hline $8 / 2 / 2009$ & 730 & INGEOMINAS \\
\hline $8 / 14 / 2009$ & 860 & INGEOMINAS \\
\hline 9/7/2009 & 230 & INGEOMINAS \\
\hline $9 / 11 / 2009$ & 300 & INGEOMINAS \\
\hline 9/14/2009 & 480 & INGEOMINAS \\
\hline 9/17/2009 & 220 & INGEOMINAS \\
\hline 9/30/2009 & 9300 & INGEOMINAS \\
\hline
\end{tabular}


Table C.1 Continued

\begin{tabular}{|c|c|c|c|}
\hline $10 / 1 / 2009$ & 10000 & & INGEOMINAS \\
\hline $10 / 2 / 2009$ & 755 & & INGEOMINAS \\
\hline $10 / 3 / 2009$ & 503 & & INGEOMINAS \\
\hline $10 / 7 / 2009$ & 90 & & INGEOMINAS \\
\hline $10 / 23 / 2009$ & 650 & & INGEOMINAS \\
\hline 10/16/2009 & 140 & & INGEOMINAS \\
\hline 10/17/2009 & 390 & & INGEOMINAS \\
\hline $10 / 25 / 2009$ & 370 & & INGEOMINAS \\
\hline $11 / 21 / 2009$ & 700 & & INGEOMINAS \\
\hline $11 / 23 / 2009$ & 120 & & INGEOMINAS \\
\hline $12 / 1 / 2009$ & 370 & & INGEOMINAS \\
\hline $12 / 2 / 2009$ & 217 & & INGEOMINAS \\
\hline $12 / 16 / 2009$ & 270 & & INGEOMINAS \\
\hline $12 / 29 / 2009$ & 860 & & INGEOMINAS \\
\hline $1 / 3 / 2010$ & 1000 & & INGEOMINAS \\
\hline $1 / 16 / 2010$ & 500 & & INGEOMINAS \\
\hline 2/8/2010 & 350 & & INGEOMINAS \\
\hline $2 / 16 / 2010$ & 303 & & INGEOMINAS \\
\hline $3 / 2 / 2010$ & 137 & & INGEOMINAS \\
\hline $3 / 4 / 2010$ & 102 & & INGEOMINAS \\
\hline $3 / 16 / 2010$ & 209 & & INGEOMINAS \\
\hline $3 / 18 / 2010$ & 165 & & INGEOMINAS \\
\hline $5 / 4 / 2010$ & 215 & & INGEOMINAS \\
\hline $6 / 8 / 2010$ & 20000 & & INGEOMINAS \\
\hline $6 / 15 / 2010$ & 24 & & INGEOMINAS \\
\hline $6 / 21 / 2010$ & 12 & & INGEOMINAS \\
\hline \multicolumn{4}{|l|}{ Tungurahua } \\
\hline Date & $\mathrm{SO}_{2}\left(\mathrm{t} \mathrm{d}^{-1}\right)$ & Instrument & Source \\
\hline $10 / 22 / 2000$ & 1000 & & BVGN \\
\hline $1 / 16 / 2001$ & 2200 & & BVGN \\
\hline $3 / 1 / 2001$ & 1036 & ASTER & This Study \\
\hline $8 / 16 / 2001$ & 5000 & & BVGN \\
\hline $\begin{array}{l}5 / 13 / 2002- \\
5 / 28 / 2002\end{array}$ & 950 & & BVGN \\
\hline $6 / 29 / 2002$ & 1800 & DOAS & Barrancos et al. 2008 \\
\hline $2 / 23 / 2005$ & 1200 & & BVGN \\
\hline $4 / 11 / 2005$ & 1600 & & BVGN \\
\hline
\end{tabular}


Table C. 1 Continued

\begin{tabular}{|c|c|c|c|}
\hline 7/21/2006 & 604 & DOAS & Barrancos et al. 2008 \\
\hline 7/22/2006 & 1809 & DOAS & Barrancos et al. 2008 \\
\hline $7 / 23 / 2006$ & 3191 & DOAS & Barrancos et al. 2008 \\
\hline $7 / 25 / 2006$ & 3727 & DOAS & Barrancos et al. 2008 \\
\hline $7 / 26 / 2006$ & 3094 & DOAS & Barrancos et al. 2008 \\
\hline $7 / 27 / 2006$ & 4601 & DOAS & Barrancos et al. 2008 \\
\hline $7 / 28 / 2006$ & 2374 & DOAS & Barrancos et al. 2008 \\
\hline 7/29/2006 & 1241 & DOAS & Barrancos et al. 2008 \\
\hline $7 / 30 / 2006$ & 3569 & DOAS & Barrancos et al. 2008 \\
\hline $1 / 6 / 2010$ & 3200 & & BVGN \\
\hline \multicolumn{4}{|c|}{ Nevado de Huilia } \\
\hline Date & $\mathrm{SO}_{2}\left(\mathrm{t} \mathrm{d}^{-1}\right)$ & Instrument & Source \\
\hline $5 / 5 / 2007$ & 3000 & & BVGN \\
\hline $5 / 28 / 2007$ & 3000 & & BVGN \\
\hline $6 / 1 / 2007$ & 6900 & & BVGN \\
\hline $10 / 26 / 2008$ & 7793 & & BVGN \\
\hline $10 / 27 / 2008$ & 4551 & & BVGN \\
\hline $10 / 28 / 2008$ & 3766 & & BVGN \\
\hline $2 / 10 / 2010$ & 945 & & BVGN \\
\hline $2 / 16 / 2010$ & 4130 & & BVGN \\
\hline \multicolumn{4}{|l|}{ Ubinas } \\
\hline Date & $\mathrm{SO}_{2}\left(\mathrm{t} \mathrm{d}^{-1}\right)$ & Instrument & Source \\
\hline $07 / 04 / 2006$ & 1589 & ASTER & This study \\
\hline $10 / 15 / 2006$ & 1139 & ASTER & This Study \\
\hline \multicolumn{4}{|l|}{ Lascar } \\
\hline Date & $\mathrm{SO}_{2}\left(\mathrm{t} \mathrm{d}^{-1}\right)$ & Instrument & Source \\
\hline $4 / 9 / 200$ & 2373 & ASTER & This study \\
\hline $10 / 1 / 2000$ & 1300 & ASTER & This study \\
\hline $10 / 9 / 2000$ & 1238 & ASTER & This study \\
\hline $11 / 9 / 2000$ & 1291 & ASTER & This study \\
\hline $12 / 18 / 2002$ & 1135 & ASTER & This study \\
\hline $1 / 18 / 2003$ & 2300 & DOAS & Mather et al. 2004 \\
\hline $4 / 2 / 2003$ & 854 & ASTER & This study \\
\hline 9/16/2003 & 936 & ASTER & This study \\
\hline $12 / 7 / 2004$ & 470 & ASTER & This study \\
\hline
\end{tabular}


Table C.1 Continued

\begin{tabular}{|c|c|c|c|}
\hline $5 / 26 / 2005$ & 277 & ASTER & This study \\
\hline $8 / 20 / 2005$ & 122 & ASTER & This study \\
\hline $8 / 7 / 2006$ & 209 & ASTER & This study \\
\hline $8 / 23 / 2006$ & 509 & ASTER & This study \\
\hline $1 / 7 / 2007$ & 188 & ASTER & This study \\
\hline $10 / 5 / 2010$ & 473 & ASTER & This study \\
\hline $11 / 6 / 2010$ & 412 & ASTER & This study \\
\hline $11 / 22 / 2010$ & 537 & ASTER & This study \\
\hline $11 / 9 / 2010$ & 581 & ASTER & This study \\
\hline $12 / 11 / 2011$ & 952 & ASTER & This study \\
\hline \multicolumn{4}{|l|}{ Villarrica } \\
\hline Date & $\mathrm{SO}_{2}\left(\mathrm{t} \mathrm{d}^{-1}\right)$ & Instrument & Source \\
\hline $1 / 29 / 2000$ & 178 & COSPEC & Witter et al. 2004 \\
\hline $1 / 20$ & 358 & COSPEC & Witter et al. 2004 \\
\hline $2 / 2 / 2000$ & 274 & COSPEC & Witter et al. 2004 \\
\hline $2 / 5 / 2000$ & 209 & COSPEC & Witter et al. 2004 \\
\hline $2 / 7 / 2000$ & 121 & COSPEC & Witter et al. 2004 \\
\hline $2 / 13 / 2000$ & 204 & COSPEC & Witter et al. 2004 \\
\hline $2 / 14 / 2000$ & 151 & COSPEC & Witter et al. 2004 \\
\hline $3 / 3 / 2000$ & 149 & COSPEC & Witter et al. 2004 \\
\hline $3 / 7 / 2000$ & 174 & COSPEC & Witter et al. 2004 \\
\hline $3 / 18 / 2000$ & 374 & COSPEC & Witter et al. 2004 \\
\hline $3 / 21 / 2000$ & 180 & COSPEC & Witter et al. 2004 \\
\hline $4 / 1 / 2000$ & 269 & COSPEC & Palma et al. 2008 \\
\hline $5 / 4 / 2000$ & 171 & COSPEC & Palma et al. 2008 \\
\hline $6 / 1 / 2000$ & 80 & COSPEC & Palma et al. 2008 \\
\hline $1 / 15 / 2001$ & 118 & COSPEC & Witter et al. 2004 \\
\hline $1 / 25 / 2001$ & 441 & COSPEC & Witter et al. 2004 \\
\hline $1 / 28 / 2001$ & 564 & COSPEC & Witter et al. 2004 \\
\hline $2 / 2 / 2001$ & 732 & COSPEC & Witter et al. 2004 \\
\hline $2 / 8 / 2003$ & 397 & COSPEC & Palma et al. 2008 \\
\hline $2 / 12 / 2003$ & 281 & COSPEC & Palma et al. 2008 \\
\hline $11 / 10 / 2004$ & 261 & COSPEC & Palma et al. 2008 \\
\hline $1 / 13 / 2005$ & 735 & COSPEC & Palma et al. 2008 \\
\hline $1 / 15 / 2005$ & 1299 & COSPEC & Palma et al. 2008 \\
\hline $1 / 17 / 2005$ & 951 & COSPEC & Palma et al. 2008 \\
\hline $1 / 19 / 2005$ & 603 & COSPEC & Palma et al. 2008 \\
\hline
\end{tabular}




\section{Table C.1 Continued}

\begin{tabular}{|l|r|l|l|}
\hline $1 / 24 / 2005$ & 996 & COSPEC & Palma et al. 2008 \\
\hline $1 / 17 / 2006$ & 122 & COSPEC & Palma et al. 2008 \\
\hline $2 / 9 / 2006$ & 262 & COSPEC & Palma et al. 2008 \\
\hline $1 / 13 / 2006$ & 149 & COSPEC & Palma et al. 2008 \\
\hline
\end{tabular}


OMI Data

Table C2

Fuego and Pacaya- Data processed by Dulci Avouris (2011).

\begin{tabular}{|r|r|}
\hline Date & $\begin{array}{r}\text { Mass } \\
\text { (kilotons) }\end{array}$ \\
\hline $1 / 1 / 2006$ & 0.016 \\
\hline $1 / 4 / 2006$ & 0.002 \\
\hline $1 / 6 / 2006$ & 0.004 \\
\hline $1 / 8 / 2006$ & 0.057 \\
\hline $1 / 9 / 2006$ & 0.042 \\
\hline $1 / 11 / 2006$ & 0.021 \\
\hline $1 / 12 / 2006$ & 0.002 \\
\hline $1 / 13 / 2006$ & 0.065 \\
\hline $1 / 15 / 2006$ & 0.043 \\
\hline $1 / 16 / 2006$ & 0.062 \\
\hline $1 / 17 / 2006$ & 0.01 \\
\hline $1 / 18 / 2006$ & 0.004 \\
\hline $1 / 19 / 2006$ & 0.008 \\
\hline $1 / 21 / 2006$ & 0.032 \\
\hline $1 / 22 / 2006$ & 0.037 \\
\hline $1 / 23 / 2006$ & 0.011 \\
\hline $1 / 24 / 2006$ & 0.004 \\
\hline $1 / 25 / 2006$ & 0.016 \\
\hline $1 / 27 / 2006$ & 0.009 \\
\hline $1 / 28 / 2006$ & 0.013 \\
\hline $1 / 29 / 2006$ & 0.052 \\
\hline $1 / 30 / 2006$ & 0.014 \\
\hline $2 / 1 / 2006$ & 0.029 \\
\hline $2 / 2 / 2006$ & 0.105 \\
\hline $2 / 3 / 2006$ & 0.005 \\
\hline $2 / 6 / 2006$ & 0.046 \\
\hline $2 / 7 / 2006$ & 0.065 \\
\hline $2 / 10 / 2006$ & 0.014 \\
\hline $2 / 12 / 2006$ & 0.009 \\
\hline $2 / 13 / 2006$ & 0.022 \\
\hline $2 / 14 / 2006$ & 0.079 \\
\hline $2 / 15 / 2006$ & 0.004 \\
\hline $2 / 16 / 2006$ & 0.041 \\
\hline & \\
\hline
\end{tabular}




\begin{tabular}{|r|r|}
\hline $2 / 17 / 2006$ & 0.002 \\
\hline $2 / 18 / 2006$ & 0.014 \\
\hline $2 / 19 / 2006$ & 0.006 \\
\hline $2 / 20 / 2006$ & 0.013 \\
\hline $2 / 21 / 2006$ & 0.01 \\
\hline $2 / 22 / 2006$ & 0.008 \\
\hline $2 / 23 / 2006$ & 0.042 \\
\hline $2 / 24 / 2006$ & 0.006 \\
\hline $2 / 25 / 2006$ & 0.004 \\
\hline $2 / 26 / 2006$ & 0.003 \\
\hline $3 / 5 / 2006$ & 0.003 \\
\hline $3 / 6 / 2006$ & 0.009 \\
\hline $3 / 7 / 2006$ & 0.003 \\
\hline $3 / 8 / 2006$ & 0.003 \\
\hline $3 / 10 / 2006$ & 0.011 \\
\hline $3 / 11 / 2006$ & 0.094 \\
\hline $3 / 12 / 2006$ & 0.007 \\
\hline $3 / 13 / 2006$ & 0.02 \\
\hline $3 / 14 / 2006$ & 0.003 \\
\hline $3 / 16 / 2006$ & 0.003 \\
\hline $3 / 17 / 2006$ & 0.01 \\
\hline $3 / 18 / 2006$ & 0.012 \\
\hline $3 / 19 / 2006$ & 0.006 \\
\hline $3 / 20 / 2006$ & 0.061 \\
\hline $3 / 21 / 2006$ & 0.004 \\
\hline $3 / 23 / 2006$ & 0.002 \\
\hline $3 / 25 / 2006$ & 0.004 \\
\hline $3 / 27 / 2006$ & 0.002 \\
\hline $3 / 29 / 2006$ & 0.006 \\
\hline $3 / 30 / 2006$ & 0.002 \\
\hline $4 / 1 / 2006$ & 0.006 \\
\hline $4 / 3 / 2006$ & 0.027 \\
\hline $4 / 4 / 2006$ & 0.003 \\
\hline $4 / 5 / 2006$ & 0.014 \\
\hline $4 / 6 / 2006$ & 0.009 \\
\hline $4 / 8 / 2006$ & 0.002 \\
\hline $4 / 15 / 2006$ & 0.004 \\
\hline $4 / 28 / 2006$ & 0.016 \\
\hline & \\
\hline
\end{tabular}

Table C.2 Continued 


\begin{tabular}{|r|r|}
\hline $5 / 7 / 2006$ & 0.059 \\
\hline $5 / 22 / 2006$ & 0.058 \\
\hline $5 / 23 / 2006$ & 0.276 \\
\hline $5 / 24 / 2006$ & 0.017 \\
\hline $5 / 25 / 2006$ & 0.432 \\
\hline $5 / 26 / 2006$ & 0.057 \\
\hline $5 / 28 / 2006$ & 0.006 \\
\hline $5 / 30 / 2006$ & 0.036 \\
\hline $5 / 31 / 2006$ & 0.017 \\
\hline $6 / 2 / 2006$ & 0.003 \\
\hline $6 / 11 / 2006$ & 0.453 \\
\hline $6 / 12 / 2006$ & 0.022 \\
\hline $6 / 13 / 2006$ & 0.001 \\
\hline $6 / 22 / 2006$ & 0.012 \\
\hline $6 / 25 / 2006$ & 0.032 \\
\hline $6 / 27 / 2006$ & 0.283 \\
\hline $7 / 1 / 2006$ & 0.037 \\
\hline $7 / 2 / 2006$ & 0.003 \\
\hline $7 / 6 / 2006$ & 0.007 \\
\hline $7 / 8 / 2006$ & 0.017 \\
\hline $7 / 11 / 2006$ & 0.008 \\
\hline $7 / 22 / 2006$ & 0.023 \\
\hline $7 / 26 / 2006$ & 0.013 \\
\hline $8 / 2 / 2006$ & 0.003 \\
\hline $8 / 7 / 2006$ & 0.008 \\
\hline $8 / 9 / 2006$ & 0.004 \\
\hline $8 / 12 / 2006$ & 0.004 \\
\hline $8 / 23 / 2006$ & 0.006 \\
\hline $8 / 25 / 2006$ & 0.003 \\
\hline $8 / 28 / 2006$ & 0.003 \\
\hline $8 / 30 / 2006$ & 0.006 \\
\hline $9 / 3 / 2006$ & 0.007 \\
\hline $9 / 10 / 2006$ & 0.004 \\
\hline $9 / 13 / 2006$ & 0.098 \\
\hline $9 / 19 / 2006$ & 0.006 \\
\hline $9 / 22 / 2006$ & 0.002 \\
\hline $9 / 26 / 2006$ & 0.011 \\
\hline $9 / 29 / 2006$ & 0.02 \\
\hline & \\
\hline
\end{tabular}

Table C.2 Continued 


\begin{tabular}{|r|r|}
\hline $10 / 1 / 2006$ & 0.002 \\
\hline $10 / 5 / 2006$ & 0.008 \\
\hline $10 / 8 / 2006$ & 0.003 \\
\hline $10 / 17 / 2006$ & 0.058 \\
\hline $10 / 24 / 2006$ & 0.014 \\
\hline $10 / 26 / 2006$ & 0.009 \\
\hline $10 / 302006$ & 0.019 \\
\hline $10 / 31 / 2006$ & 0.059 \\
\hline $11 / 6 / 2006$ & 0.005 \\
\hline $11 / 7 / 2006$ & 0.01 \\
\hline $11 / 9 / 2006$ & 0.012 \\
\hline $11 / 11 / 2006$ & 0.005 \\
\hline $11 / 13 / 2006$ & 0.015 \\
\hline $11 / 14 / 2006$ & 0.007 \\
\hline $11 / 15 / 2006$ & 0.061 \\
\hline $11 / 18 / 2006$ & 0.003 \\
\hline $11 / 20 / 2006$ & 0.001 \\
\hline $11 / 22 / 2006$ & 0.061 \\
\hline $11 / 24 / 2006$ & 0.035 \\
\hline $11 / 25 / 2006$ & 0.011 \\
\hline $12 / 2 / 2006$ & 0.002 \\
\hline $12 / 4 / 2006$ & 0.005 \\
\hline $12 / 6 / 2006$ & 0.002 \\
\hline $12 / 10 / 2006$ & 0.009 \\
\hline $12 / 11 / 2006$ & 0.014 \\
\hline $12 / 13 / 2006$ & 0.006 \\
\hline $12 / 14 / 2006$ & 0.005 \\
\hline $12 / 15 / 2006$ & 0.006 \\
\hline $12 / 16 / 2006$ & 0.017 \\
\hline $12 / 17 / 2006$ & 0.007 \\
\hline $12 / 18 / 2006$ & 0.019 \\
\hline $12 / 22 / 2006$ & 0.003 \\
\hline $12 / 23 / 2006$ & 0.003 \\
\hline $12 / 24 / 2006$ & 0.002 \\
\hline $12 / 26 / 2006$ & 0.002 \\
\hline $12 / 27 / 2006$ & 0.005 \\
\hline $12 / 302006$ & 0.001 \\
\hline $12 / 312006$ & 0.022 \\
\hline & \\
\hline
\end{tabular}

Table C.2 Continued 


\begin{tabular}{|r|r|}
\hline $1 / 13 / 2007$ & 0.002 \\
\hline $1 / 14 / 2007$ & 0.003 \\
\hline $1 / 23 / 2007$ & 0.004 \\
\hline $1 / 24 / 2007$ & 0.011 \\
\hline $1 / 25 / 2007$ & 0.036 \\
\hline $1 / 28 / 2007$ & 0.012 \\
\hline $1 / 29 / 2007$ & 0.002 \\
\hline $1 / 30 / 2007$ & 0.039 \\
\hline $1 / 31 / 2007$ & 0.001 \\
\hline $2 / 1 / 2007$ & 0.023 \\
\hline $2 / 2 / 2007$ & 0.009 \\
\hline $2 / 3 / 2007$ & 0.01 \\
\hline $2 / 4 / 2007$ & 0.007 \\
\hline $2 / 6 / 2007$ & 0.006 \\
\hline $2 / 7 / 2007$ & 0.006 \\
\hline $2 / 8 / 2007$ & 0.027 \\
\hline $2 / 9 / 2007$ & 0.006 \\
\hline $2 / 10 / 2007$ & 0.012 \\
\hline $2 / 11 / 2007$ & 0.019 \\
\hline $2 / 12 / 2007$ & 0.001 \\
\hline $2 / 13 / 2007$ & 0.024 \\
\hline $2 / 14 / 2007$ & 0.003 \\
\hline $2 / 15 / 2007$ & 0.001 \\
\hline $2 / 17 / 2007$ & 0.004 \\
\hline $2 / 18 / 2007$ & 0.014 \\
\hline $2 / 19 / 2007$ & 0.023 \\
\hline $2 / 20 / 2007$ & 0.001 \\
\hline $2 / 212007$ & 0.003 \\
\hline $2 / 22 / 2007$ & 0.001 \\
\hline $2 / 23 / 2007$ & 0.001 \\
\hline $2 / 24 / 2007$ & 0.021 \\
\hline $2 / 25 / 2007$ & 0.009 \\
\hline $2 / 26 / 2007$ & 0.047 \\
\hline $2 / 28 / 2007$ & 0.012 \\
\hline $3 / 1 / 2007$ & 0.033 \\
\hline $3 / 2 / 2007$ & 0.007 \\
\hline $3 / 3 / 2007$ & 0.006 \\
\hline $3 / 4 / 2007$ & 0.002 \\
\hline & \\
\hline
\end{tabular}

Table C.2 Continued 


\begin{tabular}{|r|r|}
\hline $3 / 6 / 2007$ & 0.059 \\
\hline $3 / 7 / 2007$ & 0.023 \\
\hline $3 / 8 / 2007$ & 0.005 \\
\hline $3 / 9 / 2007$ & 0.002 \\
\hline $3 / 13 / 2007$ & 0.007 \\
\hline $3 / 14 / 2007$ & 0.076 \\
\hline $3 / 15 / 2007$ & 0.066 \\
\hline $3 / 16 / 2007$ & 0.714 \\
\hline $3 / 17 / 2007$ & 0.032 \\
\hline $3 / 19 / 2007$ & 0.009 \\
\hline $3 / 22 / 2007$ & 0.002 \\
\hline $3 / 23 / 2007$ & 0.003 \\
\hline $3 / 28 / 2007$ & 0.001 \\
\hline $4 / 2 / 2007$ & 0.002 \\
\hline $4 / 7 / 2007$ & 0.003 \\
\hline $4 / 9 / 2007$ & 0.002 \\
\hline $4 / 10 / 2007$ & 0.006 \\
\hline $4 / 11 / 2007$ & 0.008 \\
\hline $4 / 22 / 2007$ & 0.003 \\
\hline $4 / 24 / 2007$ & 0.022 \\
\hline $4 / 25 / 2007$ & 0.015 \\
\hline $5 / 1 / 2007$ & 0.067 \\
\hline $5 / 4 / 2007$ & 0.015 \\
\hline $5 / 10 / 2007$ & 0.056 \\
\hline $5 / 11 / 2007$ & 0.006 \\
\hline $5 / 18 / 2007$ & 0.005 \\
\hline $5 / 26 / 2007$ & 0.003 \\
\hline $5 / 29 / 2007$ & 0.006 \\
\hline $6 / 2 / 2007$ & 0.012 \\
\hline $6 / 4 / 2007$ & 0.024 \\
\hline $6 / 9 / 2007$ & 0.004 \\
\hline $6 / 12 / 2007$ & 0.002 \\
\hline $6 / 13 / 2007$ & 0.003 \\
\hline $6 / 14 / 2007$ & 0.024 \\
\hline $6 / 16 / 2007$ & 0.002 \\
\hline $6 / 18 / 2007$ & 0.036 \\
\hline $6 / 19 / 2007$ & 0.005 \\
\hline $6 / 20 / 2007$ & 0.018 \\
\hline & \\
\hline
\end{tabular}

Table C.2 Continued 


\begin{tabular}{|r|r|}
\hline \multicolumn{1}{|l}{} \\
\hline $6 / 26 / 2007$ & 0.012 \\
\hline $6 / 27 / 2007$ & 0.008 \\
\hline $6 / 28 / 2007$ & 0.023 \\
\hline $6 / 29 / 2007$ & 0.053 \\
\hline $6 / 30 / 2007$ & 0.015 \\
\hline $7 / 1 / 2007$ & 0.281 \\
\hline $7 / 22007$ & 0.008 \\
\hline $7 / 4 / 2007$ & 0.014 \\
\hline $7 / 7 / 2007$ & 0.002 \\
\hline $7 / 12 / 2007$ & 0.005 \\
\hline $7 / 21 / 2007$ & 0.007 \\
\hline $7 / 25 / 2007$ & 0.002 \\
\hline $7 / 27 / 2007$ & 0.021 \\
\hline $7 / 28 / 2007$ & 0.022 \\
\hline $7 / 29 / 2007$ & 0.029 \\
\hline $8 / 1 / 2007$ & 0.002 \\
\hline $8 / 7 / 2007$ & 0.008 \\
\hline $8 / 8 / 2007$ & 0.077 \\
\hline $8 / 13 / 2007$ & 0.017 \\
\hline $8 / 15 / 2007$ & 0.002 \\
\hline $8 / 16 / 2007$ & 0.019 \\
\hline $8 / 17 / 2007$ & 0.004 \\
\hline $8 / 29 / 2007$ & 0.001 \\
\hline $9 / 8 / 2007$ & 0.045 \\
\hline $9 / 14 / 2007$ & 0.02 \\
\hline $9 / 15 / 2007$ & 0.003 \\
\hline $9 / 29 / 2007$ & 0.002 \\
\hline $9 / 30 / 2007$ & 0.001 \\
\hline $10 / 22007$ & 0.004 \\
\hline $10 / 3 / 2007$ & 0.002 \\
\hline $10 / 8 / 2007$ & 0.002 \\
\hline $10 / 15 / 2007$ & 0.004 \\
\hline $10 / 17 / 2007$ & 0.003 \\
\hline $10 / 22 / 2007$ & 0.001 \\
\hline $10 / 26 / 2007$ & 0.015 \\
\hline $10 / 27 / 2007$ & 0.01 \\
\hline $10 / 29 / 2007$ & 0.002 \\
\hline $11 / 1 / 2007$ & 0.013 \\
\hline
\end{tabular}

Table C.2 Continued 


\begin{tabular}{|r|r|}
\hline $11 / 9 / 2007$ & 0.048 \\
\hline $11 / 12 / 2007$ & 0.002 \\
\hline $11 / 16 / 2007$ & 0.006 \\
\hline $11 / 17 / 2007$ & 0.003 \\
\hline $11 / 18 / 2007$ & 0.018 \\
\hline $12 / 9 / 2007$ & 0.006 \\
\hline $12 / 11 / 2007$ & 0.004 \\
\hline $12 / 13 / 2007$ & 0.003 \\
\hline $12 / 16 / 2007$ & 0.009 \\
\hline $12 / 18 / 2007$ & 0.001 \\
\hline $12 / 20 / 2007$ & 0.001 \\
\hline $12 / 23 / 2007$ & 0.002 \\
\hline $12 / 24 / 2007$ & 0.025 \\
\hline $12 / 25 / 2007$ & 0.014 \\
\hline $12 / 26 / 2007$ & 0.002 \\
\hline $12 / 27 / 2007$ & 0.051 \\
\hline $12 / 29 / 2007$ & 0.008 \\
\hline $12 / 30 / 2007$ & 0.005 \\
\hline $1 / 3 / 2008$ & 0.005 \\
\hline $1 / 4 / 2008$ & 0.011 \\
\hline $1 / 5 / 2008$ & 0.012 \\
\hline $1 / 8 / 2008$ & 0.001 \\
\hline $1 / 10 / 2008$ & 0.021 \\
\hline $1 / 11 / 2008$ & 0.009 \\
\hline $1 / 12 / 2008$ & 0.02 \\
\hline $1 / 14 / 2008$ & 0.008 \\
\hline $1 / 15 / 2008$ & 0.001 \\
\hline $1 / 16 / 2008$ & 0.004 \\
\hline $1 / 17 / 2008$ & 0.021 \\
\hline $1 / 18 / 2008$ & 0.002 \\
\hline $1 / 19 / 2008$ & 0.021 \\
\hline $1 / 20 / 2008$ & 0.053 \\
\hline $1 / 21 / 2008$ & 0.011 \\
\hline $1 / 22 / 2008$ & 0.028 \\
\hline $1 / 24 / 2008$ & 0.002 \\
\hline $1 / 25 / 2008$ & 0.014 \\
\hline $1 / 26 / 2008$ & 0.014 \\
\hline $1 / 27 / 2008$ & 0.003 \\
\hline
\end{tabular}

Table C.2 Continued 


\begin{tabular}{|r|r|}
\hline $1 / 29 / 2008$ & 0.016 \\
\hline $1 / 30 / 2008$ & 0.073 \\
\hline $1 / 31 / 2008$ & 0.017 \\
\hline $2 / 2 / 2008$ & 0.009 \\
\hline $2 / 3 / 2008$ & 0.009 \\
\hline $3 / 4 / 2008$ & 0.024 \\
\hline $2 / 5 / 2008$ & 0.074 \\
\hline $2 / 6 / 2008$ & 0.028 \\
\hline $2 / 8 / 2008$ & 0.002 \\
\hline $2 / 9 / 2008$ & 0.019 \\
\hline $2 / 11 / 2008$ & 0.011 \\
\hline $2 / 12 / 2008$ & 0.077 \\
\hline $2 / 13 / 2008$ & 0.019 \\
\hline $2 / 16 / 2008$ & 0.015 \\
\hline $2 / 18 / 2008$ & 0.009 \\
\hline $2 / 19 / 2008$ & 0.02 \\
\hline $2 / 20 / 2008$ & 0.012 \\
\hline $2 / 21 / 2008$ & 0.015 \\
\hline $2 / 22 / 2008$ & 0.018 \\
\hline $2 / 23 / 2008$ & 0.001 \\
\hline $2 / 24 / 2008$ & 0.006 \\
\hline $2 / 25 / 2008$ & 0.009 \\
\hline $2 / 26 / 2008$ & 0.003 \\
\hline $2 / 27 / 2008$ & 0.016 \\
\hline $2 / 28 / 2008$ & 0.065 \\
\hline $3 / 3 / 2008$ & 0.012 \\
\hline $3 / 5 / 2008$ & 0.002 \\
\hline $3 / 7 / 2008$ & 0.011 \\
\hline $3 / 8 / 2008$ & 0.014 \\
\hline $3 / 13 / 2008$ & 0.004 \\
\hline $3 / 14 / 2008$ & 0.095 \\
\hline $3 / 15 / 2008$ & 0.028 \\
\hline $3 / 16 / 2008$ & 0.029 \\
\hline $3 / 18 / 2008$ & 0.003 \\
\hline $3 / 19 / 2008$ & 0.003 \\
\hline $3 / 23 / 2008$ & 0.058 \\
\hline $3 / 24 / 2008$ & 0.003 \\
\hline $3 / 25 / 2008$ & 0.008 \\
\hline & \\
\hline
\end{tabular}

Table C.2 Continued 


\begin{tabular}{|r|r|}
\hline $3 / 26 / 2008$ & 0.003 \\
\hline $3 / 30 / 2008$ & 0.016 \\
\hline $4 / 1 / 2008$ & 0.034 \\
\hline $4 / 2 / 2008$ & 0.005 \\
\hline $4 / 3 / 2008$ & 0.006 \\
\hline $4 / 4 / 2008$ & 0.004 \\
\hline $4 / 15 / 2008$ & 0.005 \\
\hline $4 / 16 / 2008$ & 0.011 \\
\hline $4 / 17 / 2008$ & 0.001 \\
\hline $4 / 24 / 2008$ & 0.01 \\
\hline $4 / 25 / 2008$ & 0.01 \\
\hline $5 / 1 / 2008$ & 0.008 \\
\hline $5 / 2 / 2008$ & 0.069 \\
\hline $5 / 19 / 2008$ & 0.008 \\
\hline $5 / 24 / 2008$ & 0.008 \\
\hline $5 / 27 / 2008$ & 0.005 \\
\hline $5 / 29 / 2008$ & 0.006 \\
\hline $5 / 31 / 2008$ & 0.003 \\
\hline $6 / 1 / 2008$ & 0.13 \\
\hline $6 / 2 / 2008$ & 0.169 \\
\hline $6 / 3 / 2008$ & 0.285 \\
\hline $6 / 4 / 2008$ & 0.153 \\
\hline $6 / 5 / 2008$ & 0.16 \\
\hline $6 / 6 / 2008$ & 0.197 \\
\hline $6 / 7 / 2008$ & 0.348 \\
\hline $6 / 8 / 2008$ & 0.098 \\
\hline $6 / 9 / 2008$ & 0.227 \\
\hline $6 / 10 / 2008$ & 0.104 \\
\hline $6 / 11 / 2008$ & 0.089 \\
\hline $6 / 12 / 2008$ & 0.275 \\
\hline $6 / 13 / 2008$ & 0.246 \\
\hline $6 / 14 / 2008$ & 0.195 \\
\hline $6 / 15 / 2008$ & 0.237 \\
\hline $6 / 16 / 2008$ & 0.231 \\
\hline $6 / 17 / 2008$ & 0.056 \\
\hline $6 / 18 / 2008$ & 0.271 \\
\hline $6 / 19 / 2008$ & 0.05 \\
\hline $6 / 20 / 2008$ & 0.065 \\
\hline & \\
\hline
\end{tabular}

Table C.2 Continued 


\begin{tabular}{|r|r|}
\hline $6 / 21 / 2008$ & 0.053 \\
\hline $6 / 22 / 2008$ & 0.3 \\
\hline $6 / 23 / 2008$ & 0.386 \\
\hline $6 / 24 / 2008$ & 0.091 \\
\hline $6 / 25 / 2008$ & 0.409 \\
\hline $6 / 26 / 2008$ & 0.116 \\
\hline $6 / 27 / 2008$ & 0.147 \\
\hline $6 / 28 / 2008$ & 0.232 \\
\hline $6 / 29 / 2008$ & 0.198 \\
\hline $6 / 30 / 2008$ & 0.392 \\
\hline $7 / 5 / 2008$ & 0.002 \\
\hline $7 / 10 / 2008$ & 0.013 \\
\hline $7 / 15 / 2008$ & 0.004 \\
\hline $8 / 16 / 2008$ & 0.007 \\
\hline $8 / 17 / 2008$ & 0.012 \\
\hline $8 / 21 / 2008$ & 0.011 \\
\hline $8 / 22 / 2008$ & 0.015 \\
\hline $8 / 30 / 2008$ & 0.011 \\
\hline $9 / 7 / 2008$ & 0.015 \\
\hline $9 / 9 / 2008$ & 0.005 \\
\hline $9 / 17 / 2008$ & 0.004 \\
\hline $9 / 23 / 2008$ & 0.004 \\
\hline $10 / 1 / 2008$ & 0.038 \\
\hline $10 / 10 / 2008$ & 0.003 \\
\hline $10 / 24 / 2008$ & 0.002 \\
\hline $10 / 25 / 2008$ & 0.019 \\
\hline $11 / 2 / 2008$ & 0.002 \\
\hline $11 / 3 / 2008$ & 0.003 \\
\hline $11 / 4 / 2008$ & 0.022 \\
\hline $11 / 5 / 2008$ & 0.02 \\
\hline $11 / 7 / 2008$ & 0.008 \\
\hline $11 / 9 / 2008$ & 0.034 \\
\hline $11 / 10 / 2008$ & 0.046 \\
\hline $11 / 11 / 2008$ & 0.004 \\
\hline $11 / 12 / 2008$ & 0.003 \\
\hline $11 / 16 / 2008$ & 0.001 \\
\hline $11 / 19 / 2008$ & 0.002 \\
\hline $11 / 20 / 2008$ & 0.011 \\
\hline & \\
\hline
\end{tabular}

Table C.2 Continued 


\begin{tabular}{|r|r|}
\hline $11 / 23 / 2008$ & 0.002 \\
\hline $11 / 25 / 2008$ & 0.007 \\
\hline $11 / 26 / 2008$ & 0.039 \\
\hline $11 / 27 / 2008$ & 0.021 \\
\hline $11 / 30 / 2008$ & 0.006 \\
\hline $12 / 1 / 2008$ & 0.004 \\
\hline $12 / 22008$ & 0.013 \\
\hline $12 / 5 / 2008$ & 0.005 \\
\hline $12 / 9 / 2008$ & 0.001 \\
\hline $12 / 11 / 2008$ & 0.047 \\
\hline $12 / 12 / 2008$ & 0.028 \\
\hline $12 / 13 / 2008$ & 0.019 \\
\hline $12 / 15 / 2008$ & 0.011 \\
\hline $12 / 16 / 2008$ & 0.002 \\
\hline $12 / 18 / 2008$ & 0.008 \\
\hline $12 / 21 / 2008$ & 0.002 \\
\hline $12 / 23 / 2008$ & 0.001 \\
\hline $12 / 25 / 2008$ & 0.002 \\
\hline $12 / 27 / 2008$ & 0.012 \\
\hline $1 / 1 / 2009$ & 0.004 \\
\hline $1 / 2 / 2009$ & 0.004 \\
\hline $1 / 3 / 2009$ & 0.011 \\
\hline $1 / 4 / 2009$ & 0.003 \\
\hline $1 / 5 / 2009$ & 0.014 \\
\hline $1 / 6 / 2009$ & 0.002 \\
\hline $1 / 13 / 2009$ & 0.129 \\
\hline $1 / 14 / 2009$ & 0.002 \\
\hline $1 / 15 / 2009$ & 0.008 \\
\hline $1 / 19 / 2009$ & 0.007 \\
\hline $1 / 20 / 2009$ & 0.001 \\
\hline $1 / 21 / 2009$ & 0.031 \\
\hline $1 / 22 / 2009$ & 0.012 \\
\hline $1 / 23 / 2009$ & 0.08 \\
\hline $1 / 26 / 2009$ & 0.327 \\
\hline $1 / 28 / 2009$ & 0.02 \\
\hline $2 / 4 / 2009$ & 0.004 \\
\hline $2 / 5 / 2009$ & 0.124 \\
\hline $2 / 6 / 2009$ & 0.016 \\
\hline
\end{tabular}

Table C.2 Continued 


\begin{tabular}{|r|r|}
\hline $2 / 7 / 2009$ & 0.026 \\
\hline $2 / 8 / 2009$ & 0.002 \\
\hline $2 / 10 / 2009$ & 0.003 \\
\hline $2 / 11 / 2009$ & 0.003 \\
\hline $2 / 13 / 2009$ & 0.018 \\
\hline $2 / 14 / 2009$ & 0.088 \\
\hline $2 / 15 / 2009$ & 0.007 \\
\hline $2 / 16 / 2009$ & 0.015 \\
\hline $2 / 17 / 2009$ & 0.014 \\
\hline $2 / 19 / 2009$ & 0.042 \\
\hline $2 / 21 / 2009$ & 0.002 \\
\hline $2 / 22 / 2009$ & 0.003 \\
\hline $2 / 23 / 2009$ & 0.014 \\
\hline $2 / 24 / 2009$ & 0.002 \\
\hline $2 / 26 / 2009$ & 0.004 \\
\hline $3 / 1 / 2009$ & 0.001 \\
\hline $3 / 2 / 2009$ & 0.004 \\
\hline $3 / 3 / 2009$ & 0.019 \\
\hline $3 / 5 / 2009$ & 0.003 \\
\hline $3 / 7 / 2009$ & 0.002 \\
\hline $3 / 8 / 2009$ & 0.008 \\
\hline $3 / 9 / 2009$ & 0.002 \\
\hline $3 / 10 / 2009$ & 0.005 \\
\hline $3 / 12 / 2009$ & 0.07 \\
\hline $3 / 15 / 2009$ & 0.012 \\
\hline $3 / 16 / 2009$ & 0.034 \\
\hline $3 / 17 / 2009$ & 0.005 \\
\hline $3 / 18 / 2009$ & 0.018 \\
\hline $3 / 20 / 2009$ & 0.289 \\
\hline $3 / 24 / 2009$ & 0.015 \\
\hline $3 / 25 / 2009$ & 0.001 \\
\hline $3 / 26 / 2009$ & 0.005 \\
\hline $3 / 27 / 2009$ & 0.004 \\
\hline $3 / 28 / 2009$ & 0.004 \\
\hline $4 / 2 / 2009$ & 0.049 \\
\hline $4 / 3 / 2009$ & 0.01 \\
\hline $4 / 4 / 2009$ & 0.008 \\
\hline $4 / 9 / 2009$ & 0.006 \\
\hline
\end{tabular}

Table C.2 Continued 


\begin{tabular}{|r|r|}
\hline $4 / 10 / 2009$ & 0.003 \\
\hline $4 / 11 / 2009$ & 0.017 \\
\hline $4 / 13 / 2009$ & 0.043 \\
\hline $4 / 16 / 2009$ & 0.001 \\
\hline $4 / 18 / 2009$ & 0.016 \\
\hline $4 / 19 / 2009$ & 0.024 \\
\hline $4 / 21 / 2009$ & 0.004 \\
\hline $4 / 26 / 2009$ & 0.002 \\
\hline $5 / 4 / 2009$ & 0.014 \\
\hline $5 / 5 / 2009$ & -0.002 \\
\hline $5 / 11 / 2009$ & 0.001 \\
\hline $5 / 13 / 2009$ & 0.043 \\
\hline $5 / 16 / 2009$ & 0.021 \\
\hline $5 / 28 / 2009$ & 0.049 \\
\hline $5 / 29 / 2009$ & 0.037 \\
\hline $6 / 16 / 2009$ & 0.013 \\
\hline $6 / 28 / 2009$ & 0.001 \\
\hline $7 / 2 / 2009$ & 0.077 \\
\hline $7 / 3 / 2009$ & 0.001 \\
\hline $7 / 16 / 2009$ & 0.009 \\
\hline $7 / 23 / 2009$ & 0.003 \\
\hline $7 / 24 / 2009$ & 0.021 \\
\hline $7 / 27 / 2009$ & 0.005 \\
\hline $7 / 31 / 2009$ & 0.013 \\
\hline $8 / 8 / 2009$ & 0.009 \\
\hline $8 / 9 / 2009$ & 0.028 \\
\hline $8 / 24 / 2009$ & 0.001 \\
\hline $8 / 25 / 2009$ & 0.001 \\
\hline $8 / 31 / 2009$ & 0.001 \\
\hline $9 / 4 / 2009$ & 0.008 \\
\hline $9 / 5 / 2009$ & 0.005 \\
\hline $9 / 72009$ & 0.029 \\
\hline $9 / 10 / 2009$ & 0.081 \\
\hline $9 / 11 / 2009$ & 0.031 \\
\hline $9 / 18 / 2009$ & 0.021 \\
\hline $9 / 26 / 2009$ & 0.031 \\
\hline $9 / 29 / 2009$ & 0.003 \\
\hline $10 / 3 / 2009$ & 0.007 \\
\hline & \\
\hline
\end{tabular}

Table C.2 Continued 


\begin{tabular}{|r|r|}
\hline $10 / 4 / 2009$ & 0.043 \\
\hline $10 / 22 / 2009$ & 0.011 \\
\hline $10 / 27 / 2009$ & 0.005 \\
\hline $10 / 28 / 2009$ & 0.032 \\
\hline $10 / 29 / 2009$ & 0.008 \\
\hline $11 / 3 / 2009$ & 0.002 \\
\hline $11 / 12 / 2009$ & 0.045 \\
\hline $11 / 13 / 2009$ & 0.003 \\
\hline $11 / 14 / 2009$ & 0.048 \\
\hline $11 / 19 / 2009$ & 0.021 \\
\hline $11 / 20 / 2009$ & 0.004 \\
\hline $11 / 21 / 2009$ & 0.04 \\
\hline $11 / 23 / 2009$ & 0.004 \\
\hline $11 / 26 / 2009$ & 0.012 \\
\hline $11 / 28 / 2009$ & 0.037 \\
\hline $11 / 129 / 2009$ & 0.015 \\
\hline $11 / 30 / 2009$ & 0.037 \\
\hline $12 / 1 / 2009$ & 0.003 \\
\hline $12 / 4 / 2009$ & 0.001 \\
\hline $12 / 5 / 2009$ & 0.002 \\
\hline $12 / 6 / 2009$ & 0.009 \\
\hline $12 / 7 / 2009$ & 0.015 \\
\hline $12 / 9 / 2009$ & 0.028 \\
\hline $12 / 12 / 2009$ & 0.027 \\
\hline $12 / 13 / 2009$ & 0.001 \\
\hline $12 / 14 / 2009$ & 0.002 \\
\hline $12 / 15 / 2009$ & 0.001 \\
\hline $12 / 21 / 2009$ & 0.003 \\
\hline $12 / 23 / 2009$ & 0.041 \\
\hline $12 / 28 / 2009$ & 0.028 \\
\hline $12 / 30 / 2009$ & 0.003 \\
\hline $12 / 31 / 2009$ & 0.05 \\
\hline $1 / 6 / 2010$ & 0.006 \\
\hline $1 / 7 / 2010$ & 0.022 \\
\hline $1 / 8 / 2010$ & 0.007 \\
\hline $1 / 12 / 2010$ & 0.021 \\
\hline $1 / 17 / 2010$ & 0.019 \\
\hline $1 / 19 / 2010$ & 0.001 \\
\hline
\end{tabular}

Table C.2 Continued 


\begin{tabular}{|r|r|}
\hline $1 / 21 / 2010$ & 0.008 \\
\hline $1 / 22 / 2010$ & 0.002 \\
\hline $1 / 23 / 2010$ & 0.052 \\
\hline $1 / 24 / 2010$ & 0.025 \\
\hline $1 / 28 / 2010$ & 0.008 \\
\hline $1 / 31 / 2010$ & 0.007 \\
\hline $2 / 1 / 2010$ & 0.005 \\
\hline $2 / 2 / 2010$ & 0.001 \\
\hline $2 / 6 / 2010$ & 0.142 \\
\hline $2 / 7 / 2010$ & 0.152 \\
\hline $2 / 8 / 2010$ & 0.053 \\
\hline $2 / 9 / 2010$ & 0.097 \\
\hline $2 / 11 / 2010$ & 0.032 \\
\hline $2 / 12 / 2010$ & 0.013 \\
\hline $2 / 13 / 2010$ & 0.004 \\
\hline $2 / 15 / 2010$ & 0.047 \\
\hline $2 / 16 / 2010$ & 0.027 \\
\hline $2 / 17 / 2010$ & 0.069 \\
\hline $2 / 23 / 2010$ & 0.001 \\
\hline $2 / 25 / 2010$ & 0.036 \\
\hline $2 / 27 / 2010$ & 0.009 \\
\hline $2 / 28 / 2010$ & 0.013 \\
\hline $3 / 3 / 2010$ & 0.05 \\
\hline $3 / 4 / 2010$ & 0.031 \\
\hline $3 / 5 / 2010$ & 0.082 \\
\hline $3 / 6 / 2010$ & 0.055 \\
\hline $3 / 8 / 2010$ & 0.008 \\
\hline $3 / 13 / 2010$ & 0.054 \\
\hline $3 / 15 / 2010$ & 0.063 \\
\hline $3 / 20 / 2010$ & 0.051 \\
\hline $3 / 21 / 2010$ & 0.181 \\
\hline $3 / 22 / 2010$ & 0.005 \\
\hline $3 / 24 / 2010$ & 0.004 \\
\hline $3 / 27 / 2010$ & 0.008 \\
\hline $3 / 28 / 2010$ & 0.003 \\
\hline $3 / 29 / 2010$ & 0.009 \\
\hline $3 / 31 / 2010$ & 0.022 \\
\hline $4 / 3 / 2010$ & 0.002 \\
\hline & \\
\hline
\end{tabular}

Table C.2 Continued 


\begin{tabular}{|r|r|}
\hline $4 / 4 / 2010$ & 0.004 \\
\hline $4 / 5 / 2010$ & 0.062 \\
\hline $4 / 6 / 2010$ & 0.086 \\
\hline $4 / 7 / 2010$ & 0.006 \\
\hline $4 / 30 / 2010$ & 0.097 \\
\hline $4 / 2 / 2010$ & 0.013 \\
\hline $5 / 3 / 2010$ & -0.001 \\
\hline $5 / 4 / 2010$ & 0.005 \\
\hline $5 / 6 / 2010$ & 0.011 \\
\hline $5 / 7 / 2010$ & 0.001 \\
\hline $5 / 13 / 2010$ & 0.027 \\
\hline $5 / 14 / 2010$ & 0.002 \\
\hline $5 / 15 / 2010$ & 0.032 \\
\hline $5 / 16 / 2010$ & 0.077 \\
\hline $5 / 19 / 2010$ & 0.022 \\
\hline $5 / 23 / 2010$ & 0.002 \\
\hline $5 / 24 / 2010$ & 0.156 \\
\hline $5 / 25 / 2010$ & 0.002 \\
\hline $5 / 31 / 2010$ & 0.096 \\
\hline $6 / 1 / 2010$ & 0.276 \\
\hline $6 / 3 / 2010$ & 0.025 \\
\hline $6 / 4 / 2010$ & 0.008 \\
\hline $6 / 6 / 2010$ & 0.026 \\
\hline $6 / 8 / 2010$ & 0.021 \\
\hline $6 / 9 / 2010$ & 0.037 \\
\hline $6 / 10 / 2010$ & 0.018 \\
\hline $6 / 11 / 2010$ & 0.016 \\
\hline $6 / 12 / 2010$ & 0.003 \\
\hline $6 / 15 / 2010$ & 0.011 \\
\hline $6 / 24 / 2010$ & 0.03 \\
\hline $6 / 25 / 2010$ & 0.175 \\
\hline $7 / 1 / 2010$ & 0.039 \\
\hline $7 / 2 / 2010$ & 0.004 \\
\hline $7 / 5 / 2010$ & 0.01 \\
\hline $7 / 10 / 2010$ & 0.009 \\
\hline $7 / 11 / 2010$ & 0.15 \\
\hline $7 / 13 / 2010$ & 0.005 \\
\hline $7 / 17 / 2010$ & 0.006 \\
\hline & \\
\hline
\end{tabular}

Table C.2 Continued 
Table C.2 Continued

\begin{tabular}{|r|r|}
\hline $7 / 24 / 2010$ & 0.009 \\
\hline $7 / 26 / 2010$ & 0.052 \\
\hline $7 / 27 / 2010$ & 0.053 \\
\hline $7 / 28 / 2010$ & 0.011 \\
\hline $8 / 2 / 2010$ & 0.001 \\
\hline $8 / 3 / 2010$ & 0.073 \\
\hline $8 / 7 / 2010$ & 0.014 \\
\hline $8 / 11 / 2010$ & 0.004 \\
\hline $8 / 12 / 2010$ & 0.032 \\
\hline $8 / 28 / 2010$ & 0.047 \\
\hline $8 / 29 / 2010$ & 0.032 \\
\hline $8 / 31 / 2010$ & 0.024 \\
\hline $9 / 5 / 2010$ & 0.018 \\
\hline $9 / 7 / 2010$ & 0.029 \\
\hline $9 / 10 / 2010$ & 0.003 \\
\hline $9 / 3 / 2010$ & 0.1 \\
\hline $9 / 19 / 2010$ & 0.004 \\
\hline $9 / 21 / 2010$ & 0.007 \\
\hline $9 / 28 / 2010$ & 0.012 \\
\hline $9 / 29 / 2010$ & 0.058 \\
\hline $10 / 5 / 2010$ & 0.008 \\
\hline $10 / 9 / 2010$ & 0.003 \\
\hline $10 / 12 / 2010$ & 0.001 \\
\hline $10 / 15 / 2010$ & 0.061 \\
\hline $10 / 15 / 2010$ & 0.011 \\
\hline $10 / 23 / 2010$ & 0.013 \\
\hline $10 / 20 / 2010$ & 0.012 \\
\hline $10 / 31 / 2010$ & 0.13 \\
\hline & \\
\hline
\end{tabular}

Santa Ana

\begin{tabular}{|l|l|}
\hline Date & Flux t d $^{-1}$ \\
\hline $08 / 25 / 2005$ & 376 \\
\hline $10 / 01 / 2005$ & 12537 \\
\hline
\end{tabular}

Turrialba

\begin{tabular}{|l|l|}
\hline Date & Flux t d $^{-1}$ \\
\hline $03 / 14 / 2009$ & 297 \\
\hline $05 / 01 / 2009$ & 490 \\
\hline $05 / 15 / 2009$ & 194 \\
\hline
\end{tabular}


Table C.2 Continued

\begin{tabular}{|l|l|}
\hline $05 / 17 / 2009$ & 297 \\
\hline $05 / 19 / 2009$ & 376 \\
\hline $06 / 23 / 2009$ & 296 \\
\hline $09 / 14 / 2009$ & 287 \\
\hline $10 / 31 / 2009$ & 414 \\
\hline $11 / 07 / 2009$ & 1070 \\
\hline $11 / 17 / 2009$ & 288 \\
\hline $01 / 10 / 2010$ & 352 \\
\hline $01 / 21 / 2010$ & 707 \\
\hline $01 / 26 / 2010$ & 380 \\
\hline $03 / 08 / 2010$ & 731 \\
\hline $03 / 12 / 2010$ & 695 \\
\hline $04 / 06 / 2010$ & 2132 \\
\hline $05 / 03 / 2010$ & 1569 \\
\hline $05 / 04 / 2010$ & 378 \\
\hline $05 / 06 / 2010$ & 379 \\
\hline $05 / 27 / 2010$ & 522 \\
\hline $07 / 06 / 2010$ & 1729 \\
\hline $08 / 06 / 2010$ & 1516 \\
\hline $08 / 17 / 2010$ & 823 \\
\hline $08 / 21 / 2010$ & 554 \\
\hline $08 / 23 / 2010$ & \\
\hline $08 / 22 / 2010$ & 5236 \\
\hline $08 / 31 / 2010$ & 1124 \\
\hline $09 / 04 / 2010$ & 887 \\
\hline $09 / 07 / 2010$ & 1352 \\
\hline $09 / 16 / 2010$ & 408 \\
\hline $09 / 18 / 2010$ & 2565 \\
\hline $10 / 08 / 2010$ & 3703 \\
\hline $10 / 09 / 2010$ & 5164 \\
\hline $10 / 11 / 2010$ & 1311 \\
\hline $10 / 20 / 2010$ & 1168 \\
\hline $10 / 29 / 2020$ & 624 \\
\hline $11 / 05 / 2010$ & 825 \\
\hline $11 / 12 / 2010$ & 1069 \\
\hline & \\
\hline & \\
\hline
\end{tabular}

Ubinas

\begin{tabular}{|l|l|}
\hline Date & Flux t d $^{-1}$ \\
\hline $03 / 31 / 2006$ & 1200 \\
\hline $05 / 08 / 2006$ & 996 \\
\hline $07 / 04 / 2006$ & 301 \\
\hline
\end{tabular}


Table C.2 Continued

\begin{tabular}{|l|l|}
\hline $10 / 15 / 2006$ & 958 \\
\hline $06 / 02 / 2007$ & 806 \\
\hline $06 / 29 / 2007$ & 705 \\
\hline $04 / 02 / 2008$ & 802 \\
\hline
\end{tabular}

El Misit

\begin{tabular}{|l|l|}
\hline Date & Flux t d $^{-1}$ \\
\hline $02 / 16 / 2010$ & 674 \\
\hline
\end{tabular}

Table C3

Villarrica OMI data - Source Dulci Avouris (2011)

\begin{tabular}{|r|r|}
\hline \multicolumn{1}{|l|}{ Date } & \multicolumn{1}{|r|}{$\begin{array}{l}\mathrm{SO}_{2} \text { mass } \\
\text { (kilo tons) }\end{array}$} \\
\hline $1 / 32006$ & 0.005 \\
\hline $1 / 19 / 2006$ & 0.015 \\
\hline $1 / 20 / 2006$ & 0.009 \\
\hline $1 / 21 / 2006$ & 0.005 \\
\hline $1 / 23 / 2006$ & 0.005 \\
\hline $1 / 25 / 2006$ & 0.003 \\
\hline $1 / 29 / 2006$ & 0.004 \\
\hline $2 / 1 / 2006$ & 0.006 \\
\hline $2 / 2 / 2006$ & 0.012 \\
\hline $2 / 5 / 2006$ & 0.002 \\
\hline $2 / 6 / 2006$ & 0.003 \\
\hline $2 / 6 / 2006$ & 0.006 \\
\hline $2 / 11 / 2006$ & 0.04 \\
\hline $2 / 13 / 2006$ & 0.014 \\
\hline $2 / 18 / 2006$ & 0.004 \\
\hline $2 / 20 / 2006$ & 0.021 \\
\hline $2 / 23 / 2006$ & 0.005 \\
\hline $2 / 24 / 2006$ & 0.004 \\
\hline $2 / 25 / 2006$ & 0.007 \\
\hline $3 / 3 / 2006$ & 0.003 \\
\hline $3 / 4 / 2006$ & 0.015 \\
\hline $3 / 9 / 2006$ & 0.003 \\
\hline $3 / 11 / 2006$ & 0.004 \\
\hline $2 / 13 / 2006$ & 0.005 \\
\hline $3 / 18 / 2006$ & 0.019 \\
\hline & \\
\hline
\end{tabular}




\begin{tabular}{|r|r|}
\hline $3 / 20 / 2006$ & 0.017 \\
\hline $3 / 22 / 2006$ & 0.002 \\
\hline $3 / 24 / 2006$ & 0.009 \\
\hline $3 / 27 / 2006$ & 0.068 \\
\hline $3 / 28 / 2006$ & 0.014 \\
\hline $3 / 29 / 2006$ & 0.074 \\
\hline $3 / 31 / 2006$ & 0.043 \\
\hline $4 / 1 / 2006$ & 0.038 \\
\hline $4 / 2 / 2006$ & 0.027 \\
\hline $4 / 3 / 2006$ & 0.02 \\
\hline $4 / 4 / 2006$ & 0.006 \\
\hline $4 / 5 / 2006$ & 0.06 \\
\hline $4 / 6 / 2006$ & 0.003 \\
\hline $4 / 7 / 2006$ & 0.085 \\
\hline $4 / 8 / 2006$ & 0.019 \\
\hline $4 / 9 / 2006$ & 0.097 \\
\hline $4 / 12 / 2006$ & 0.012 \\
\hline $4 / 14 / 2006$ & 0.038 \\
\hline $4 / 15 / 2006$ & 0.021 \\
\hline $4 / 16 / 2006$ & 0.204 \\
\hline $4 / 19 / 2006$ & 0.005 \\
\hline $4 / 21 / 2006$ & 0.005 \\
\hline $4 / 23 / 2006$ & 0.051 \\
\hline $4 / 25 / 2006$ & 0.03 \\
\hline $4 / 26 / 2006$ & 0.009 \\
\hline $4 / 27 / 2006$ & 0.017 \\
\hline $4 / 28 / 2006$ & 0.048 \\
\hline $4 / 29 / 2006$ & 0.004 \\
\hline $4 / 30 / 2006$ & 0.055 \\
\hline $5 / 1 / 2006$ & 0.097 \\
\hline $5 / 2 / 2006$ & 0.066 \\
\hline $5 / 3 / 2006$ & 0.011 \\
\hline $5 / 4 / 2006$ & 0.02 \\
\hline $5 / 5 / 2006$ & 0.181 \\
\hline $5 / 6 / 2006$ & 0.042 \\
\hline $5 / 7 / 2006$ & 0.122 \\
\hline $5 / 8 / 2006$ & 0.045 \\
\hline $5 / 9 / 2006$ & 0.091 \\
\hline & \\
\hline
\end{tabular}

Table C.2 Continued 


\begin{tabular}{|r|r|}
\hline $5 / 10 / 2006$ & 0.089 \\
\hline $5 / 11 / 2006$ & 0.082 \\
\hline $5 / 12 / 2006$ & 0.058 \\
\hline $5 / 13 / 2006$ & 0.024 \\
\hline $5 / 14 / 2006$ & 0.04 \\
\hline $5 / 15 / 2006$ & 0.014 \\
\hline $5 / 16 / 2006$ & 0.037 \\
\hline $5 / 18 / 2006$ & 0.013 \\
\hline $5 / 21 / 2006$ & 0.043 \\
\hline $5 / 23 / 2006$ & 0.028 \\
\hline $5 / 25 / 2006$ & 0.03 \\
\hline $5 / 26 / 2006$ & 0.003 \\
\hline $5 / 27 / 2006$ & 0.033 \\
\hline $5 / 28 / 2006$ & 0.065 \\
\hline $5 / 31 / 2006$ & 0.04 \\
\hline $6 / 1 / 2006$ & 0.03 \\
\hline $6 / 3 / 2006$ & 0.017 \\
\hline $6 / 4 / 2006$ & 0.019 \\
\hline $6 / 5 / 2006$ & 0.007 \\
\hline $6 / 6 / 2006$ & 0.097 \\
\hline $6 / 8 / 2006$ & 0.023 \\
\hline $6 / 13 / 2006$ & 0.018 \\
\hline $6 / 15 / 2006$ & 0.075 \\
\hline $6 / 21 / 2006$ & 0.015 \\
\hline $6 / 22 / 2006$ & 0.031 \\
\hline $6 / 24 / 2006$ & 0.007 \\
\hline $6 / 26 / 2006$ & 0.008 \\
\hline $6 / 28 / 2006$ & 0.006 \\
\hline $6 / 29 / 2006$ & 0.003 \\
\hline $7 / 1 / 2006$ & 0.047 \\
\hline $7 / 3 / 2006$ & 0.02 \\
\hline $7 / 5 / 2006$ & 0.012 \\
\hline $7 / 6 / 2006$ & 0.009 \\
\hline $7 / 19 / 2006$ & 0.026 \\
\hline $7 / 28 / 2006$ & 0.009 \\
\hline $7 / 30 / 2006$ & 0.12 \\
\hline $7 / 31 / 2006$ & 0.063 \\
\hline $8 / 1 / 2006$ & 0.027 \\
\hline & \\
\hline
\end{tabular}

Table C.2 Continued 


\begin{tabular}{|r|r|}
\hline $8 / 2 / 2006$ & 0.005 \\
\hline $8 / 4 / 2006$ & 0.002 \\
\hline $8 / 14 / 2006$ & 0.003 \\
\hline $8 / 15 / 2006$ & 0.014 \\
\hline $8 / 16 / 2006$ & 0.035 \\
\hline $8 / 17 / 2006$ & 0.034 \\
\hline $8 / 18 / 2006$ & 0.058 \\
\hline $8 / 20 / 2006$ & 0.09 \\
\hline $8 / 21 / 2006$ & 0.015 \\
\hline $8 / 22 / 2006$ & 0.047 \\
\hline $8 / 23 / 2006$ & 0.079 \\
\hline $8 / 27 / 2006$ & 0.008 \\
\hline $8 / 29 / 2006$ & 0.002 \\
\hline $9 / 3 / 2006$ & 0.007 \\
\hline $9 / 12 / 2006$ & 0.017 \\
\hline $9 / 14 / 2006$ & 0.012 \\
\hline $9 / 17 / 2006$ & 0.057 \\
\hline $9 / 18 / 2006$ & 0.002 \\
\hline $9 / 19 / 2006$ & 0.01 \\
\hline $9 / 23 / 2006$ & 0.015 \\
\hline $9 / 24 / 2006$ & 0.003 \\
\hline $10 / 16 / 2006$ & 0.003 \\
\hline $10 / 18 / 2006$ & 0.014 \\
\hline $10 / 30 / 2006$ & 0.017 \\
\hline $11 / 3 / 2006$ & 0.003 \\
\hline $11 / 6 / 2006$ & 0.009 \\
\hline $11 / 8 / 2006$ & 0.03 \\
\hline $11 / 9 / 2006$ & 0.006 \\
\hline $11 / 15 / 2006$ & 0.003 \\
\hline $11 / 17 / 2006$ & 0.047 \\
\hline $11 / 19 / 2006$ & 0.048 \\
\hline $11 / 26 / 2006$ & 0.005 \\
\hline $12 / 5 / 2006$ & 0.002 \\
\hline $12 / 7 / 2006$ & 0.006 \\
\hline $12 / 8 / 2006$ & 0.003 \\
\hline $12 / 11 / 2006$ & 0.003 \\
\hline $12 / 30 / 2006$ & 0.004 \\
\hline $1 / 2 / 2007$ & 0.004 \\
\hline
\end{tabular}

Table C.2 Continued 


\begin{tabular}{|r|r|}
\hline $1 / 6 / 2007$ & 0.008 \\
\hline $1 / 7 / 2007$ & 0.002 \\
\hline $1 / 8 / 2007$ & 0.018 \\
\hline $1 / 9 / 2007$ & 0.004 \\
\hline $1 / 12 / 2007$ & 0.009 \\
\hline $1 / 15 / 2007$ & 0.011 \\
\hline $1 / 18 / 2007$ & 0.002 \\
\hline $1 / 27 / 2007$ & 0.003 \\
\hline $1 / 31 / 2007$ & 0.009 \\
\hline $2 / 1 / 2007$ & 0.014 \\
\hline $2 / 7 / 2007$ & 0.004 \\
\hline $2 / 12 / 2007$ & -0.001 \\
\hline $2 / 14 / 2007$ & 0.004 \\
\hline $2 / 17 / 2007$ & 0.004 \\
\hline $2 / 18 / 2007$ & 0.016 \\
\hline $2 / 19 / 2007$ & 0.016 \\
\hline $2 / 22 / 2007$ & 0.003 \\
\hline $2 / 23 / 2007$ & 0.003 \\
\hline $2 / 25 / 2007$ & 0.075 \\
\hline $2 / 26 / 2007$ & 0.001 \\
\hline $3 / 2 / 2007$ & 0.005 \\
\hline $3 / 3 / 2007$ & 0.006 \\
\hline $3 / 4 / 2007$ & 0.005 \\
\hline $3 / 5 / 2007$ & 0.003 \\
\hline $3 / 7 / 2007$ & 0.002 \\
\hline $3 / 9 / 2007$ & 0.009 \\
\hline $3 / 12 / 2007$ & 0.02 \\
\hline $3 / 14 / 2007$ & 0.001 \\
\hline $3 / 16 / 2007$ & 0.03 \\
\hline $3 / 17 / 2007$ & 0.002 \\
\hline $3 / 18 / 2007$ & 0.002 \\
\hline $3 / 20 / 2007$ & 0.006 \\
\hline $3 / 21 / 2007$ & 0.015 \\
\hline $3 / 23 / 2007$ & 0.042 \\
\hline $3 / 24 / 2007$ & 0.002 \\
\hline $3 / 25 / 2007$ & 0.02 \\
\hline $3 / 27 / 2007$ & 0.053 \\
\hline $3 / 28 / 2007$ & 0.012 \\
\hline & \\
\hline
\end{tabular}

Table C.2 Continued 


\begin{tabular}{|r|r|}
\hline $3 / 30 / 2007$ & 0.037 \\
\hline $4 / 1 / 2007$ & 0.006 \\
\hline $4 / 2 / 2007$ & 0.003 \\
\hline $4 / 3 / 2007$ & 0.032 \\
\hline $4 / 4 / 2007$ & 0.009 \\
\hline $4 / 5 / 2007$ & 0.021 \\
\hline $4 / 6 / 2007$ & 0.019 \\
\hline $4 / 8 / 2007$ & 0.024 \\
\hline $4 / 11 / 2007$ & 0.002 \\
\hline $4 / 12 / 2007$ & 0.001 \\
\hline $4 / 13 / 2007$ & 0.008 \\
\hline $4 / 15 / 2007$ & 0.022 \\
\hline $4 / 19 / 2007$ & 0.002 \\
\hline $4 / 20 / 2007$ & 0.041 \\
\hline $4 / 22 / 2007$ & 0.012 \\
\hline $4 / 24 / 2007$ & 0.026 \\
\hline $4 / 25 / 2007$ & 0.022 \\
\hline $4 / 26 / 2007$ & 0.091 \\
\hline $4 / 27 / 2007$ & 0.038 \\
\hline $4 / 28 / 2007$ & 0.046 \\
\hline $5 / 1 / 2007$ & 0.006 \\
\hline $5 / 5 / 2007$ & 0.005 \\
\hline $5 / 7 / 2007$ & 0.003 \\
\hline $5 / 8 / 2007$ & 0.055 \\
\hline $5 / 10 / 2007$ & 0.094 \\
\hline $5 / 11 / 2007$ & 0.03 \\
\hline $5 / 12 / 2007$ & 0.016 \\
\hline $5 / 15 / 2007$ & 0.03 \\
\hline $5 / 17 / 2007$ & 0.049 \\
\hline $5 / 19 / 2007$ & 0.133 \\
\hline $5 / 23 / 2007$ & 0.038 \\
\hline $5 / 24 / 2007$ & 0.133 \\
\hline $5 / 26 / 2007$ & 0.131 \\
\hline $5 / 27 / 2007$ & 0.012 \\
\hline $5 / 28 / 2007$ & -0.002 \\
\hline $5 / 31 / 2007$ & 0.033 \\
\hline $6 / 2 / 2007$ & 0.036 \\
\hline $6 / 4 / 2007$ & 0.046 \\
\hline & \\
\hline
\end{tabular}

Table C.2 Continued 


\begin{tabular}{|r|r|}
\hline & \\
\hline $6 / 5 / 2007$ & 0.033 \\
\hline $6 / 9 / 2007$ & 0.01 \\
\hline $6 / 11 / 2007$ & 0.037 \\
\hline $6 / 14 / 2007$ & 0.138 \\
\hline $6 / 15 / 2007$ & 0.005 \\
\hline $6 / 16 / 2007$ & 0.032 \\
\hline $6 / 18 / 2007$ & 0.125 \\
\hline $6 / 20 / 2007$ & 0.026 \\
\hline $6 / 22 / 2007$ & 0.003 \\
\hline $6 / 24 / 2007$ & 0.018 \\
\hline $6 / 25 / 2007$ & 0.317 \\
\hline $6 / 26 / 2007$ & 0.014 \\
\hline $6 / 27 / 2007$ & 0.036 \\
\hline $6 / 28 / 2007$ & 0.125 \\
\hline $6 / 29 / 2007$ & 0.079 \\
\hline $6 / 30 / 2007$ & 0.056 \\
\hline $7 / 3 / 2007$ & 0.002 \\
\hline $7 / 4 / 2007$ & 0.024 \\
\hline $7 / 9 / 2007$ & 0.036 \\
\hline $7 / 10 / 2007$ & 0.004 \\
\hline $7 / 11 / 2007$ & 0.018 \\
\hline $7 / 13 / 2007$ & 0.004 \\
\hline $7 / 14 / 2007$ & 0.017 \\
\hline $7 / 15 / 2007$ & 0.004 \\
\hline $7 / 18 / 2007$ & 0.033 \\
\hline $7 / 20 / 2007$ & 0.014 \\
\hline $7 / 23 / 2007$ & 0.004 \\
\hline $7 / 27 / 2007$ & 0.005 \\
\hline $7 / 29 / 2007$ & 0.001 \\
\hline $8 / 1 / 2007$ & 0.012 \\
\hline $8 / 2 / 2007$ & 0.009 \\
\hline $8 / 3 / 2007$ & 0.024 \\
\hline $8 / 5 / 2007$ & 0.005 \\
\hline $8 / 8 / 2007$ & 0.092 \\
\hline $8 / 10 / 2007$ & 0.005 \\
\hline $8 / 12 / 2007$ & 0.033 \\
\hline $8 / 14 / 2007$ & 0.011 \\
\hline $8 / 15 / 2007$ & 0.125 \\
\hline
\end{tabular}

Table C.2 Continued 


\begin{tabular}{|r|r|}
\hline $8 / 16 / 2007$ & 0.01 \\
\hline $8 / 18 / 2007$ & 0.006 \\
\hline $8 / 21 / 2007$ & 0.015 \\
\hline $8 / 22 / 2007$ & 0.001 \\
\hline $8 / 23 / 2007$ & 0.001 \\
\hline $8 / 30 / 2007$ & 0.038 \\
\hline $8 / 31 / 2007$ & 0.026 \\
\hline $9 / 1 / 2007$ & 0.012 \\
\hline $9 / 8 / 2007$ & 0.006 \\
\hline $9 / 9 / 2007$ & 0.002 \\
\hline $9 / 10 / 2007$ & 0.013 \\
\hline $9 / 11 / 2007$ & 0.005 \\
\hline $9 / 12 / 2007$ & 0.002 \\
\hline $9 / 13 / 2007$ & 0.02 \\
\hline $9 / 15 / 2007$ & 0.015 \\
\hline $9 / 21 / 2007$ & 0.004 \\
\hline $9 / 22 / 2007$ & 0.008 \\
\hline $9 / 23 / 2007$ & 0.016 \\
\hline $9 / 24 / 2007$ & 0.029 \\
\hline $9 / 25 / 2007$ & 0.001 \\
\hline $9 / 26 / 2007$ & 0.008 \\
\hline $9 / 27 / 2007$ & 0.007 \\
\hline $9 / 28 / 2007$ & 0.014 \\
\hline $9 / 29 / 2007$ & 0.001 \\
\hline $10 / 1 / 2007$ & 0.019 \\
\hline $10 / 8 / 2007$ & 0.006 \\
\hline $10 / 10 / 2007$ & 0.034 \\
\hline $10 / 11 / 2007$ & 0.019 \\
\hline $10 / 17 / 2007$ & 0.003 \\
\hline $10 / 21 / 2007$ & 0.01 \\
\hline $10 / 23 / 2007$ & 0.004 \\
\hline $11 / 6 / 2007$ & 0.004 \\
\hline $11 / 19 / 2007$ & 0.003 \\
\hline $11 / 20 / 2007$ & 0.007 \\
\hline $11 / 22 / 2007$ & 0.003 \\
\hline $11 / 25 / 2007$ & 0.014 \\
\hline $11 / 27 / 2007$ & 0.005 \\
\hline $11 / 28 / 2007$ & -0.006 \\
\hline & \\
\hline
\end{tabular}

Table C.2 Continued 


\begin{tabular}{|r|r|}
\hline $11 / 29 / 2007$ & 0.009 \\
\hline $12 / 15 / 2007$ & 0.049 \\
\hline $12 / 16 / 2007$ & 0.007 \\
\hline $12 / 18 / 2007$ & 0.018 \\
\hline $12 / 21 / 2007$ & 0.004 \\
\hline $12 / 22 / 2007$ & 0.005 \\
\hline $12 / 24 / 2007$ & 0.031 \\
\hline $12 / 28 / 2007$ & 0.002 \\
\hline $12 / 31 / 2007$ & 0.001 \\
\hline $1 / 6 / 2008$ & 0.01 \\
\hline $1 / 7 / 2008$ & 0.009 \\
\hline $1 / 14 / 2008$ & 0.002 \\
\hline $1 / 24 / 2008$ & 0.049 \\
\hline $1 / 30 / 2008$ & 0.038 \\
\hline $1 / 31 / 2008$ & 0.01 \\
\hline $2 / 1 / 2008$ & 0.016 \\
\hline $2 / 2 / 2008$ & 0.014 \\
\hline $2 / 3 / 2008$ & 0.027 \\
\hline $2 / 4 / 2008$ & 0.036 \\
\hline $2 / 6 / 2008$ & 0.027 \\
\hline $2 / 7 / 2008$ & 0.267 \\
\hline $2 / 8 / 2008$ & 0.003 \\
\hline $2 / 15 / 2008$ & 0.002 \\
\hline $2 / 16 / 2008$ & 0.003 \\
\hline $2 / 17 / 2008$ & 0.014 \\
\hline $2 / 22 / 2008$ & 0.025 \\
\hline $2 / 24 / 2008$ & 0.001 \\
\hline $2 / 26 / 2008$ & 0.006 \\
\hline $2 / 27 / 2008$ & 0.01 \\
\hline $2 / 28 / 2008$ & 0.014 \\
\hline $3 / 1 / 2008$ & 0.002 \\
\hline $3 / 2 / 2008$ & 0.027 \\
\hline $3 / 6 / 2008$ & 0.052 \\
\hline $3 / 9 / 2008$ & 0.004 \\
\hline $3 / 10 / 2008$ & 0.033 \\
\hline $3 / 11 / 2008$ & 0.011 \\
\hline $3 / 13 / 2008$ & 0.01 \\
\hline $3 / 14 / 2008$ & 0.001 \\
\hline
\end{tabular}

Table C.2 Continued 


\begin{tabular}{|r|r|}
\hline $3 / 16 / 2008$ & 0.003 \\
\hline $3 / 18 / 2008$ & 0.011 \\
\hline $3 / 20 / 2008$ & 0.003 \\
\hline $3 / 21 / 2008$ & 0.001 \\
\hline $3 / 22 / 2008$ & 0.003 \\
\hline $3 / 23 / 2008$ & 0.088 \\
\hline $3 / 25 / 2008$ & 0.006 \\
\hline $3 / 26 / 2008$ & 0.021 \\
\hline $3 / 27 / 2008$ & 0.079 \\
\hline $3 / 28 / 2008$ & 0.024 \\
\hline $3 / 29 / 2008$ & 0.004 \\
\hline $3 / 30 / 2008$ & 0.006 \\
\hline $4 / 1 / 2008$ & 0.075 \\
\hline $4 / 2 / 2008$ & 0.057 \\
\hline $4 / 3 / 2008$ & 0.058 \\
\hline $4 / 5 / 2008$ & 0.035 \\
\hline $4 / 6 / 2008$ & 0.002 \\
\hline $4 / 8 / 2008$ & 0.003 \\
\hline $4 / 11 / 2008$ & 0.001 \\
\hline $4 / 13 / 2008$ & 0.017 \\
\hline $4 / 15 / 2008$ & 0.098 \\
\hline $4 / 17 / 2008$ & 0.037 \\
\hline $4 / 19 / 2008$ & 0.022 \\
\hline $4 / 22 / 2008$ & 0.01 \\
\hline $4 / 23 / 2008$ & 0.006 \\
\hline $4 / 24 / 2008$ & 0.103 \\
\hline $4 / 26 / 2008$ & 0.029 \\
\hline $4 / 27 / 2008$ & 0.067 \\
\hline $4 / 28 / 2008$ & 0.004 \\
\hline $4 / 29 / 2008$ & 0.015 \\
\hline $5 / 1 / 2008$ & 0.035 \\
\hline $5 / 2 / 2008$ & 1.66 \\
\hline $5 / 3 / 2008$ & 0.129 \\
\hline $5 / 5 / 2008$ & 0.056 \\
\hline $5 / 6 / 2008$ & 0.007 \\
\hline $5 / 7 / 2008$ & 0.031 \\
\hline $5 / 8 / 2008$ & 0.07 \\
\hline $5 / 10 / 2008$ & 0.097 \\
\hline
\end{tabular}

Table C.2 Continued 


\begin{tabular}{|r|r|}
\hline $5 / 12 / 2008$ & 0.086 \\
\hline $5 / 14 / 2008$ & 0.026 \\
\hline $5 / 15 / 2008$ & 0.009 \\
\hline $5 / 17 / 2008$ & 0.129 \\
\hline $5 / 19 / 2008$ & 0.004 \\
\hline $5 / 21 / 2008$ & 0.008 \\
\hline $5 / 24 / 2008$ & 0.098 \\
\hline $5 / 26 / 2008$ & 0.031 \\
\hline $5 / 28 / 2008$ & 0.083 \\
\hline $5 / 29 / 2008$ & 0.021 \\
\hline $5 / 30 / 2008$ & 0.063 \\
\hline $5 / 31 / 2008$ & 0.062 \\
\hline $6 / 1 / 2008$ & -0.001 \\
\hline $6 / 2 / 2008$ & 0.015 \\
\hline $6 / 4 / 2008$ & 0.002 \\
\hline $6 / 6 / 2008$ & 0.016 \\
\hline $6 / 9 / 2008$ & 0.036 \\
\hline $6 / 11 / 2008$ & 0.057 \\
\hline $6 / 13 / 2008$ & 0.048 \\
\hline $6 / 14 / 2008$ & 0.05 \\
\hline $6 / 15 / 2008$ & 0.177 \\
\hline $6 / 16 / 2008$ & 0.114 \\
\hline $6 / 18 / 2008$ & 0.01 \\
\hline $6 / 20 / 2008$ & 0.02 \\
\hline $6 / 22 / 2008$ & 0.045 \\
\hline $6 / 23 / 2008$ & 0.016 \\
\hline $6 / 24 / 2008$ & 0.001 \\
\hline $6 / 25 / 2008$ & 0.042 \\
\hline $6 / 27 / 2008$ & 0.075 \\
\hline $6 / 29 / 2008$ & 0.01 \\
\hline $6 / 30 / 2008$ & 0.013 \\
\hline $7 / 1 / 2008$ & 0.016 \\
\hline $7 / 2 / 2008$ & 0.014 \\
\hline $7 / 4 / 2008$ & 0.152 \\
\hline $7 / 6 / 2008$ & 0.14 \\
\hline $7 / 7 / 2008$ & 0.073 \\
\hline $7 / 8 / 2008$ & 0.014 \\
\hline $7 / 11 / 2008$ & 0.027 \\
\hline
\end{tabular}

Table C.2 Continued 


\begin{tabular}{|r|r|}
\hline $7 / 13 / 2008$ & 0.063 \\
\hline $7 / 15 / 2008$ & 0.079 \\
\hline $7 / 16 / 2008$ & 0.003 \\
\hline $7 / 17 / 2008$ & 0.002 \\
\hline $7 / 18 / 2008$ & 0.033 \\
\hline $7 / 19 / 2008$ & 0.075 \\
\hline $7 / 20 / 2008$ & 0.027 \\
\hline $7 / 22 / 2008$ & 0.03 \\
\hline $7 / 26 / 2008$ & 0.001 \\
\hline $7 / 29 / 2008$ & 0.018 \\
\hline $7 / 31 / 2008$ & 0.022 \\
\hline $8 / 1 / 2008$ & 0.002 \\
\hline $8 / 2 / 2008$ & 0.018 \\
\hline $8 / 3 / 2008$ & 0.013 \\
\hline $8 / 7 / 2008$ & 0.027 \\
\hline $8 / 8 / 2008$ & 0.071 \\
\hline $8 / 12 / 2008$ & 0.074 \\
\hline $8 / 14 / 2008$ & 0.007 \\
\hline $8 / 17 / 2008$ & 0.005 \\
\hline $8 / 21 / 2008$ & 0.013 \\
\hline $8 / 23 / 2008$ & 0.015 \\
\hline $8 / 28 / 2008$ & 0.005 \\
\hline $9 / 3 / 2008$ & 0.004 \\
\hline $9 / 8 / 2008$ & -0.002 \\
\hline $9 / 17 / 2008$ & 0.011 \\
\hline $9 / 19 / 2008$ & 0.233 \\
\hline $9 / 20 / 2008$ & 0.03 \\
\hline $9 / 21 / 2008$ & 0.021 \\
\hline $9 / 22 / 2008$ & 0.002 \\
\hline $9 / 24 / 2008$ & 0.025 \\
\hline $10 / 4 / 2008$ & 0.011 \\
\hline $10 / 5 / 2008$ & 0.046 \\
\hline $10 / 62008$ & 0.094 \\
\hline $10 / 17 / 2008$ & 0.006 \\
\hline $10 / 24 / 2008$ & 0.013 \\
\hline $10 / 26 / 2008$ & 0.01 \\
\hline $10 / 30 / 2008$ & 0.006 \\
\hline $11 / 13 / 2008$ & 0.006 \\
\hline
\end{tabular}

Table C.2 Continued 


\begin{tabular}{|r|r|}
\hline $11 / 15 / 2008$ & 0.003 \\
\hline $11 / 17 / 2008$ & 0.009 \\
\hline $11 / 24 / 2008$ & 0.002 \\
\hline $12 / 6 / 2008$ & 0.004 \\
\hline $12 / 7 / 2008$ & 0.001 \\
\hline $12 / 14 / 2008$ & 0.099 \\
\hline $12 / 15 / 2008$ & 0.024 \\
\hline $12 / 17 / 2008$ & 0.006 \\
\hline $12 / 22 / 2008$ & 0.002 \\
\hline $12 / 23 / 2008$ & 0.041 \\
\hline $12 / 242008$ & 0.008 \\
\hline $1 / 13 / 2009$ & 0.011 \\
\hline $1 / 15 / 2009$ & 0.005 \\
\hline $1 / 16 / 2009$ & 0.01 \\
\hline $1 / 20 / 2009$ & 0.002 \\
\hline $1 / 27 / 2009$ & 0.045 \\
\hline $1 / 29 / 2009$ & 0.022 \\
\hline $2 / 12009$ & 0.007 \\
\hline $2 / 16 / 2009$ & 0.035 \\
\hline $2 / 17 / 2009$ & 0.01 \\
\hline $2 / 18 / 2009$ & 0.002 \\
\hline $2 / 21 / 2009$ & 0.052 \\
\hline $2 / 23 / 2009$ & 0.016 \\
\hline $2 / 24 / 2009$ & 0.008 \\
\hline $2 / 26 / 2009$ & 0.027 \\
\hline $2 / 27 / 2009$ & 0.101 \\
\hline $2 / 28 / 2009$ & 0.005 \\
\hline $3 / 2 / 2009$ & 0.007 \\
\hline $3 / 3 / 2009$ & 0.005 \\
\hline $3 / 5 / 2009$ & 0.021 \\
\hline $3 / 12 / 2009$ & 0.018 \\
\hline $3 / 13 / 2009$ & 0.005 \\
\hline $3 / 14 / 2009$ & 0.01 \\
\hline $3 / 19 / 2009$ & 0.007 \\
\hline $3 / 20 / 2009$ & 0.037 \\
\hline $3 / 21 / 2009$ & 0.017 \\
\hline $3 / 22 / 2009$ & 0.006 \\
\hline $3 / 23 / 2009$ & 0.044 \\
\hline
\end{tabular}

Table C.2 Continued 


\begin{tabular}{|r|r|}
\hline $3 / 28 / 2009$ & 0.003 \\
\hline $3 / 29 / 2009$ & 0.001 \\
\hline $3 / 30 / 2009$ & 0.016 \\
\hline $3 / 31 / 2009$ & 0.235 \\
\hline $4 / 6 / 2009$ & 0.277 \\
\hline $4 / 8 / 2009$ & 0.073 \\
\hline $4 / 10 / 2009$ & 0.025 \\
\hline $4 / 13 / 2009$ & 0.089 \\
\hline $4 / 15 / 2009$ & 0.033 \\
\hline $4 / 17 / 2009$ & 0.018 \\
\hline $4 / 19 / 2009$ & 0.002 \\
\hline $4 / 20 / 2009$ & 0.002 \\
\hline $4 / 1 / 2009$ & 0.031 \\
\hline $4 / 22 / 2009$ & 0.006 \\
\hline $4 / 25 / 2009$ & 0.016 \\
\hline $4 / 28 / 2009$ & 0.02 \\
\hline $4 / 29 / 2009$ & 0.073 \\
\hline $4 / 302009$ & 0.038 \\
\hline $5 / 1 / 2009$ & 0.036 \\
\hline $5 / 2 / 2009$ & 0.003 \\
\hline $5 / 6 / 2009$ & 0.009 \\
\hline $5 / 82009$ & 0.022 \\
\hline $5 / 11 / 2009$ & 0.047 \\
\hline $5 / 15 / 2009$ & 0.005 \\
\hline $5 / 17 / 2009$ & 0.016 \\
\hline $5 / 22 / 2009$ & 0.005 \\
\hline $5 / 24 / 2009$ & 0.02 \\
\hline $5 / 27 / 2009$ & 0.035 \\
\hline $5 / 28 / 2009$ & 0.001 \\
\hline $5 / 31 / 2009$ & 0.05 \\
\hline $6 / 16 / 2009$ & 0.025 \\
\hline $6 / 17 / 2009$ & 0.004 \\
\hline $6 / 20 / 2009$ & 0.015 \\
\hline $6 / 23 / 2009$ & 0.071 \\
\hline $6 / 24 / 2009$ & 0.327 \\
\hline $6 / 25 / 2009$ & 0.028 \\
\hline $6 / 28 / 2009$ & 0.002 \\
\hline $7 / 2 / 2009$ & 0.017 \\
\hline
\end{tabular}

Table C.2 Continued 


\begin{tabular}{|r|r|}
\hline $7 / 3 / 2009$ & 0.008 \\
\hline $7 / 4 / 2009$ & 0.003 \\
\hline $7 / 5 / 2009$ & 0.006 \\
\hline $7 / 7 / 2009$ & 0.041 \\
\hline $7 / 11 / 2009$ & 0.005 \\
\hline $7 / 13 / 2009$ & 0.024 \\
\hline $7 / 14 / 2009$ & 0.039 \\
\hline $7 / 15 / 2009$ & 0.065 \\
\hline $7 / 18 / 2009$ & 0.001 \\
\hline $7 / 19 / 2009$ & 0.017 \\
\hline $7 / 25 / 2009$ & 0.015 \\
\hline $7 / 26 / 2009$ & 0.157 \\
\hline $7 / 27 / 2009$ & 0.019 \\
\hline $7 / 30 / 2009$ & 0.006 \\
\hline $8 / 4 / 2009$ & 0.012 \\
\hline $8 / 5 / 2009$ & 0.001 \\
\hline $8 / 6 / 2009$ & 0.009 \\
\hline $8 / 7 / 2009$ & 0.018 \\
\hline $8 / 10 / 2009$ & 0.001 \\
\hline $9 / 11 / 2009$ & 0.04 \\
\hline $9 / 15 / 2009$ & 0.007 \\
\hline $9 / 16 / 2009$ & 0.001 \\
\hline $9 / 22 / 2009$ & 0.011 \\
\hline $9 / 23 / 2009$ & 0.003 \\
\hline $9 / 27 / 2009$ & 0.058 \\
\hline $9 / 29 / 2009$ & 0.013 \\
\hline $10 / 6 / 2009$ & 0.017 \\
\hline $10 / 8 / 2009$ & 0.001 \\
\hline $12 / 1 / 2009$ & 0.001 \\
\hline $12 / 4 / 2009$ & 0.003 \\
\hline $12 / 10 / 2009$ & 0.007 \\
\hline $12 / 11 / 2009$ & 0.004 \\
\hline $12 / 20 / 2009$ & 0.011 \\
\hline $12 / 27 / 2009$ & 0.007 \\
\hline $1 / 19 / 2010$ & 0.002 \\
\hline $1 / 21 / 2010$ & 0.018 \\
\hline $1 / 23 / 2010$ & 0.002 \\
\hline $1 / 26 / 2010$ & 0.013 \\
\hline & \\
\hline
\end{tabular}

Table C.2 Continued 


\begin{tabular}{|r|r|}
\hline $1 / 27 / 2010$ & 0.002 \\
\hline $2 / 1 / 2010$ & 0.018 \\
\hline $2 / 3 / 2010$ & -0.002 \\
\hline $2 / 4 / 2010$ & 0.022 \\
\hline $2 / 7 / 2010$ & 0.005 \\
\hline $2 / 8 / 2010$ & 0.002 \\
\hline $2 / 13 / 2010$ & 0.002 \\
\hline $2 / 15 / 2010$ & 0.005 \\
\hline $2 / 20 / 2010$ & 0.029 \\
\hline $2 / 23 / 2010$ & 0.008 \\
\hline $2 / 24 / 2010$ & 0.028 \\
\hline $2 / 27 / 2010$ & 0.018 \\
\hline $2 / 28 / 2010$ & 0.003 \\
\hline $3 / 1 / 2010$ & 0.005 \\
\hline $3 / 10 / 2010$ & 0.016 \\
\hline $3 / 11 / 2010$ & 0.016 \\
\hline $3 / 12 / 2010$ & 0.024 \\
\hline $3 / 15 / 2010$ & 0.01 \\
\hline $3 / 24 / 2010$ & 0.044 \\
\hline $3 / 27 / 2010$ & 0.016 \\
\hline $3 / 31 / 2010$ & 0.001 \\
\hline $4 / 2 / 2010$ & 0.008 \\
\hline $4 / 4 / 2010$ & 0.01 \\
\hline $4 / 6 / 2010$ & 0.026 \\
\hline $4 / 8 / 2010$ & 0.065 \\
\hline $4 / 9 / 2010$ & 0.075 \\
\hline $4 / 11 / 2010$ & 0.007 \\
\hline $4 / 12 / 2010$ & 0.01 \\
\hline $4 / 13 / 2010$ & 0.02 \\
\hline $4 / 16 / 2010$ & 0.006 \\
\hline $4 / 17 / 2010$ & 0.002 \\
\hline $4 / 19 / 2010$ & 0.002 \\
\hline $4 / 23 / 2010$ & 0.005 \\
\hline $4 / 35 / 2010$ & 0.077 \\
\hline $4 / 27 / 2010$ & 0.002 \\
\hline $4 / 28 / 2010$ & 0.013 \\
\hline $5 / 2 / 2010$ & 0.003 \\
\hline $5 / 6 / 2010$ & 0.007 \\
\hline & \\
\hline
\end{tabular}

Table C.2 Continued 


\begin{tabular}{|r|r|}
\hline $5 / 8 / 2010$ & 0.027 \\
\hline $5 / 9 / 2010$ & 0.027 \\
\hline $5 / 11 / 2010$ & 0.148 \\
\hline $5 / 13 / 2010$ & 0.005 \\
\hline $5 / 14 / 2010$ & 0.008 \\
\hline $5 / 15 / 2010$ & 0.001 \\
\hline $5 / 21 / 2010$ & 0.011 \\
\hline $5 / 24 / 2010$ & 0.02 \\
\hline $5 / 25 / 2010$ & 0.004 \\
\hline $5 / 27 / 2010$ & 0.037 \\
\hline $5 / 29 / 2010$ & 0.079 \\
\hline $5 / 30 / 2010$ & 0.036 \\
\hline $5 / 31 / 2010$ & 0.048 \\
\hline $6 / 5 / 2010$ & 0.047 \\
\hline $6 / 7 / 2010$ & 0.067 \\
\hline $6 / 10 / 2010$ & 0.003 \\
\hline $6 / 12 / 2010$ & 0.133 \\
\hline $6 / 16 / 2010$ & 0.002 \\
\hline $6 / 19 / 2010$ & 0.003 \\
\hline $6 / 20 / 2010$ & -0.004 \\
\hline $7 / 5 / 2010$ & 0.004 \\
\hline $7 / 9 / 2010$ & 0.02 \\
\hline $7 / 12 / 2010$ & 0.013 \\
\hline $7 / 13 / 2010$ & -0.001 \\
\hline $7 / 14 / 2010$ & 0.012 \\
\hline $7 / 17 / 2010$ & 0.009 \\
\hline $7 / 18 / 2010$ & 0.001 \\
\hline $7 / 21 / 2010$ & 0.008 \\
\hline $7 / 22 / 2010$ & 0.01 \\
\hline $8 / 1 / 2010$ & 0.023 \\
\hline $8 / 2 / 2010$ & 0.008 \\
\hline $8 / 7 / 2010$ & 0.023 \\
\hline $8 / 8 / 2010$ & 0.004 \\
\hline $8 / 9 / 2010$ & 0.003 \\
\hline $8 / 13 / 2010$ & 0.023 \\
\hline $8 / 14 / 2010$ & 0.101 \\
\hline $8 / 19 / 2010$ & 0.03 \\
\hline $8 / 22 / 2010$ & 0.023 \\
\hline
\end{tabular}

Table C.2 Continued 


\section{Table C.2 Continued}

\begin{tabular}{|r|r|}
\hline $8 / 23 / 2010$ & 0.021 \\
\hline $8 / 31 / 2010$ & 0.017 \\
\hline $9 / 3 / 2010$ & 0.003 \\
\hline $9 / 7 / 2010$ & 0.072 \\
\hline $9 / 8 / 2010$ & 0.006 \\
\hline $9 / 15 / 2010$ & 0.002 \\
\hline $9 / 16 / 2010$ & 0.013 \\
\hline $9 / 18 / 2010$ & 0.003 \\
\hline $9 / 23 / 2010$ & 0.107 \\
\hline $9 / 24 / 2010$ & 0.04 \\
\hline $9 / 25 / 2010$ & 0.008 \\
\hline $10 / 9 / 2010$ & 0.04 \\
\hline $10 / 10 / 2010$ & 0.19 \\
\hline $10 / 11 / 2010$ & 0.213 \\
\hline $10 / 16 / 2010$ & 0.009 \\
\hline $10 / 25 / 2010$ & 0.019 \\
\hline $10 / 26 / 2010$ & 0.039 \\
\hline $10 / 31 / 2010$ & 0.024 \\
\hline
\end{tabular}


Table C3

Colombia and Ecuador OMI data

\begin{tabular}{|c|c|c|c|c|c|}
\hline & \multicolumn{5}{|c|}{$\mathrm{SO}_{2}$ in mass (Kilo tons) } \\
\hline Date & $\begin{array}{l}\text { Unknown } \\
\text { Source }\end{array}$ & Tungurahua & Reventador & Galeras & $\begin{array}{l}\text { Nevado } \\
\text { del Huilia }\end{array}$ \\
\hline $5 / 9 / 2007$ & 1.829 & & & & \\
\hline $5 / 10 / 2007$ & & 1.788 & & & \\
\hline $5 / 11 / 2007$ & 1.528 & & & & \\
\hline $5 / 12 / 2007$ & & 1.143 & & & \\
\hline $5 / 14 / 2007$ & 1.002 & & & & \\
\hline $5 / 15 / 2007$ & & 1.285 & & & \\
\hline $5 / 22 / 2007$ & & 1.265 & & & \\
\hline $5 / 24 / 2007$ & 2.023 & & & & \\
\hline $5 / 25 / 2007$ & & & & & 0.949 \\
\hline $5 / 28 / 2007$ & 1.714 & & & & \\
\hline $5 / 29 / 2007$ & & 0.949 & & & \\
\hline $5 / 30 / 2007$ & & 1.358 & & & \\
\hline $6 / 6 / 2007$ & 1.948 & & & & \\
\hline $6 / 20 / 2007$ & & 1.595 & & & \\
\hline $6 / 24 / 2007$ & & 1.569 & & & \\
\hline $6 / 29 / 2007$ & & 0.674 & & & \\
\hline $7 / 16 / 2007$ & & 0.248 & & & \\
\hline $7 / 17 / 2007$ & & 0.183 & & & \\
\hline $7 / 31 / 2007$ & & 0.064 & & & \\
\hline $8 / 7 / 2007$ & & & & 0.136 & \\
\hline $8 / 8 / 2007$ & & & & 0.005 & \\
\hline $8 / 9 / 2007$ & & & & 0.511 & \\
\hline $8 / 11 / 2007$ & & & & 0.015 & \\
\hline $8 / 14 / 2007$ & & & & 0.106 & \\
\hline $8 / 16 / 2007$ & & & & 0.12 & \\
\hline $8 / 19 / 2007$ & & & & 0.19 & \\
\hline $8 / 23 / 2007$ & 0.184 & & & & \\
\hline $8 / 25 / 2007$ & 0.379 & & & & \\
\hline $8 / 27 / 2007$ & & & & & 0.131 \\
\hline $9 / 8 / 2007$ & & 0.313 & & & \\
\hline $9 / 10 / 2007$ & & 0.661 & & & \\
\hline 9/17/2007 & & 0.076 & & & \\
\hline $9 / 22 / 2007$ & & 0.569 & & & \\
\hline
\end{tabular}


Table C.3 Continued

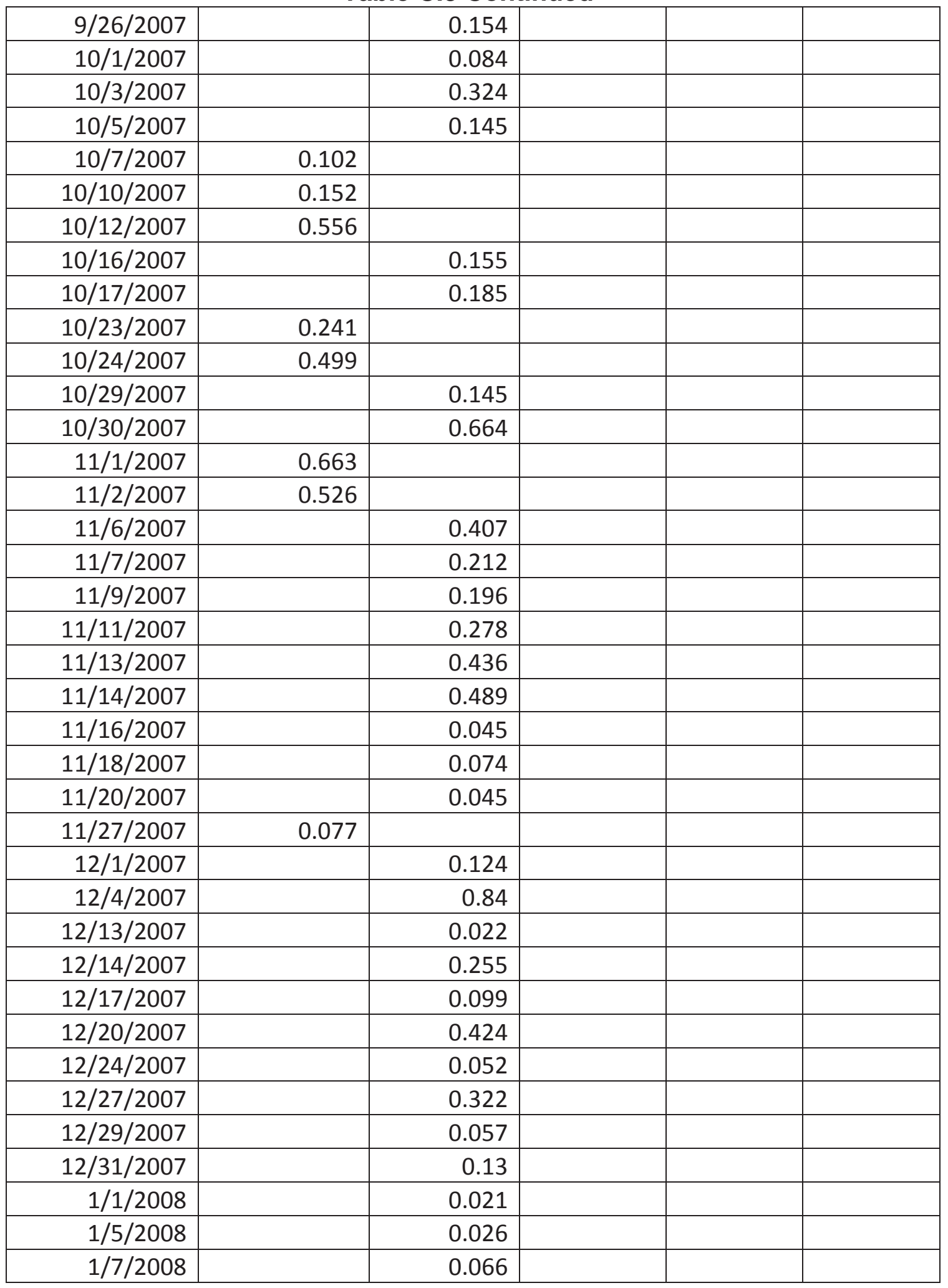


Table C.3 Continued

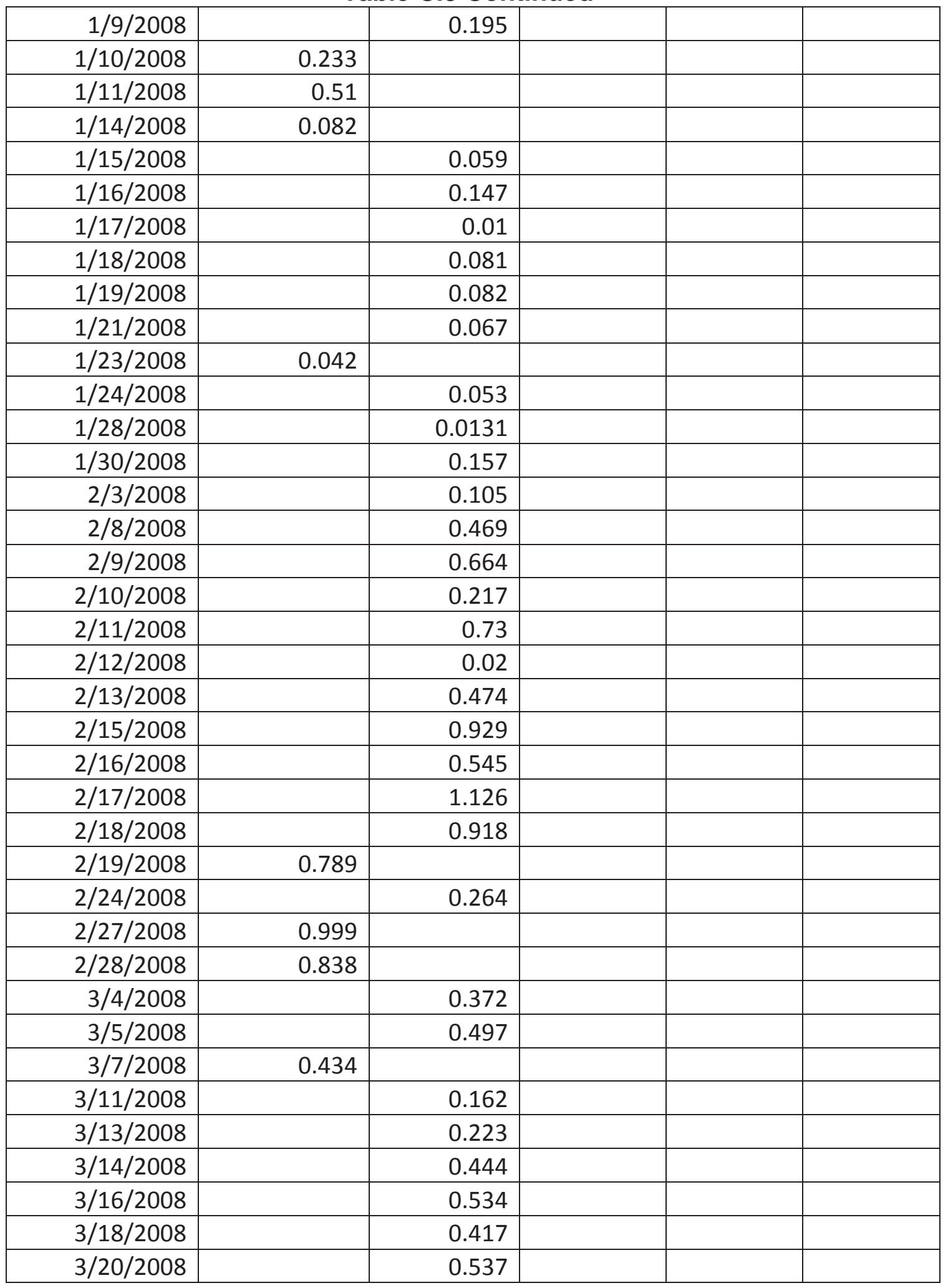


Table C.3 Continued

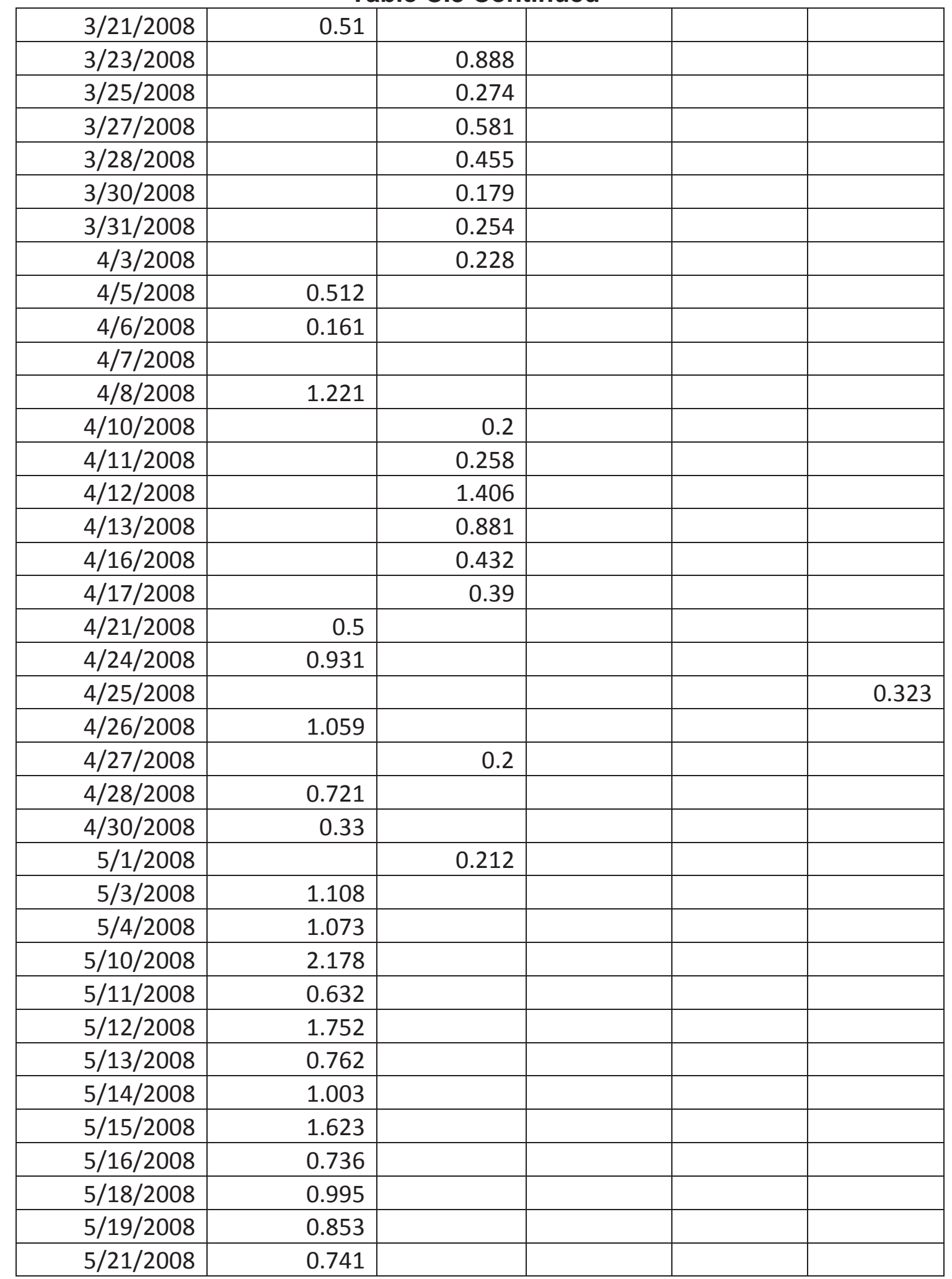


Table C.3 Continued

\begin{tabular}{|c|c|c|c|c|}
\hline $5 / 23 / 2008$ & 0.649 & & & \\
\hline $5 / 24 / 2008$ & 0.799 & & & \\
\hline $5 / 25 / 2008$ & 1.376 & & & \\
\hline $5 / 26 / 2008$ & 1.755 & & & \\
\hline $5 / 27 / 2008$ & 0.352 & & & \\
\hline $5 / 28 / 2008$ & 1.235 & & & \\
\hline $5 / 29 / 2008$ & 0.496 & & & \\
\hline $5 / 30 / 2008$ & 1.716 & & & \\
\hline $6 / 1 / 2008$ & & & & 0.917 \\
\hline $6 / 2 / 2008$ & 0.991 & & & \\
\hline $6 / 3 / 2008$ & & & & 0.24 \\
\hline $6 / 4 / 2008$ & & & & 1.229 \\
\hline $6 / 6 / 2008$ & 0.817 & & & \\
\hline $6 / 8 / 2008$ & 0.717 & & & \\
\hline $6 / 10 / 2008$ & 0.629 & & & \\
\hline $6 / 11 / 2008$ & 2.485 & & & \\
\hline $6 / 12 / 2008$ & 1.504 & & & \\
\hline $6 / 13 / 2008$ & 1.947 & & & \\
\hline $6 / 15 / 2008$ & 1.263 & & & \\
\hline $6 / 17 / 2008$ & 0.457 & & & \\
\hline $6 / 18 / 2008$ & 1.33 & & & \\
\hline $6 / 19 / 2008$ & & & 0.671 & \\
\hline $6 / 20 / 2008$ & 1.379 & & & \\
\hline $6 / 22 / 2008$ & 1.214 & & & \\
\hline $6 / 25 / 2008$ & 1.214 & & & \\
\hline $6 / 26 / 2008$ & 1.473 & & & \\
\hline $6 / 27 / 2008$ & 1.527 & & & \\
\hline $6 / 29 / 2008$ & 1.198 & & & \\
\hline $7 / 1 / 2008$ & & & & 0.385 \\
\hline $7 / 3 / 2008$ & 0.482 & & & \\
\hline $7 / 4 / 2008$ & 0.511 & & & \\
\hline $7 / 5 / 2008$ & 0.267 & & & \\
\hline $7 / 6 / 2008$ & 1.484 & & & \\
\hline $7 / 7 / 2008$ & & & & 0.429 \\
\hline $7 / 8 / 2008$ & 0.669 & & & \\
\hline $7 / 12 / 2008$ & 0.127 & & & \\
\hline $7 / 13 / 2008$ & 0.731 & & & \\
\hline $7 / 14 / 2008$ & & & & \\
\hline
\end{tabular}


Table C.3 Continued

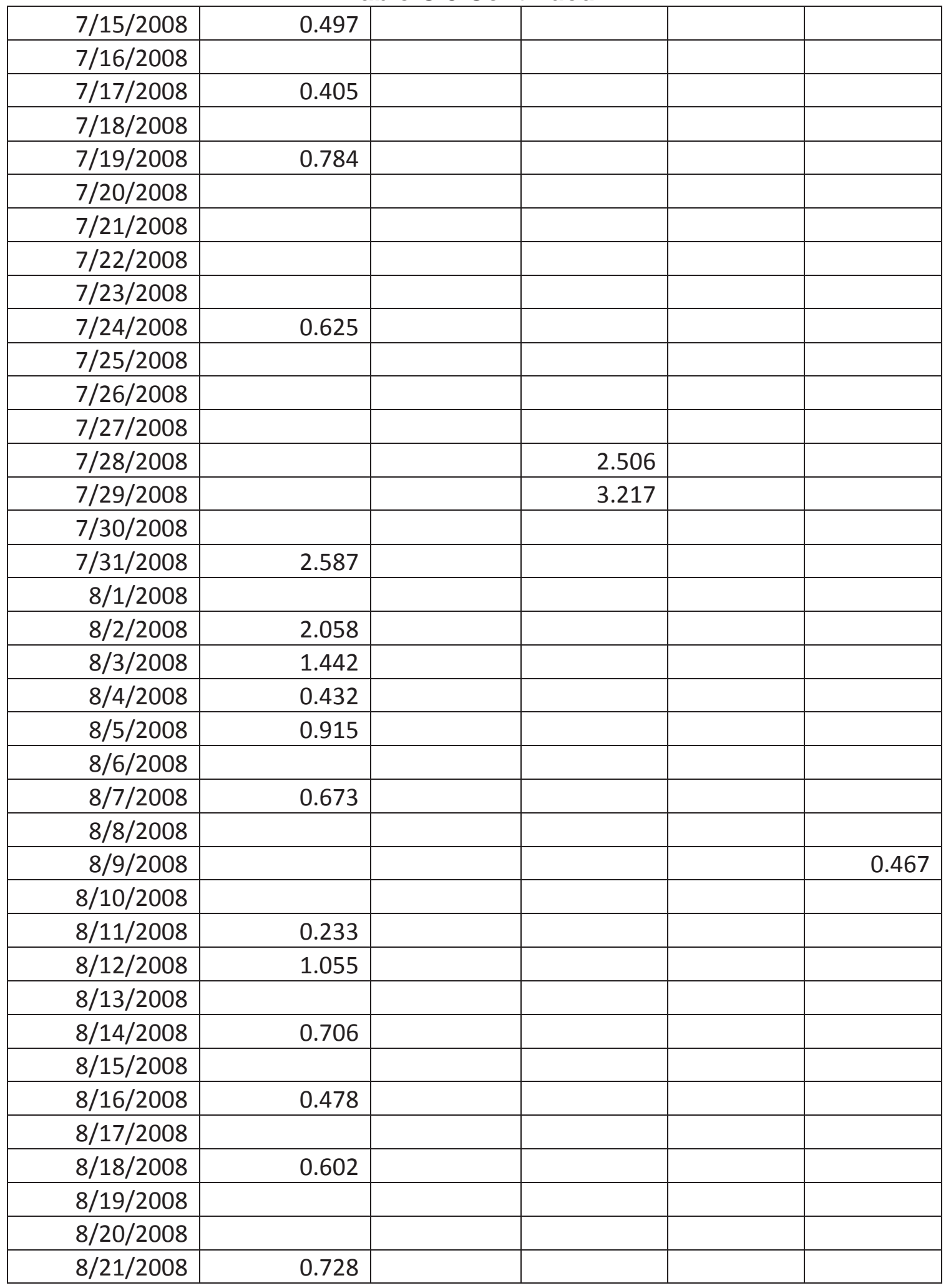


Table C.3 Continued

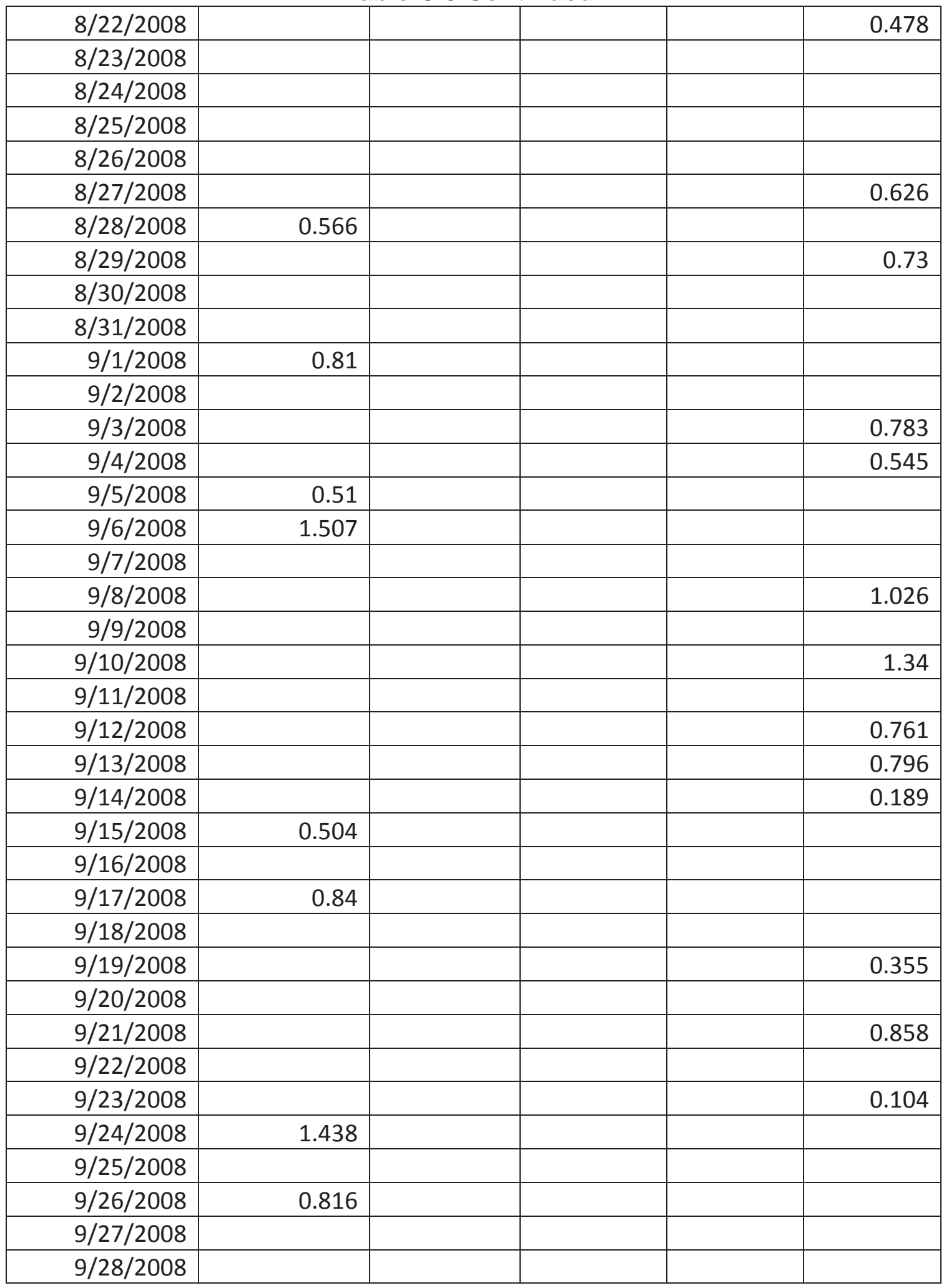


Table C.3 Continued

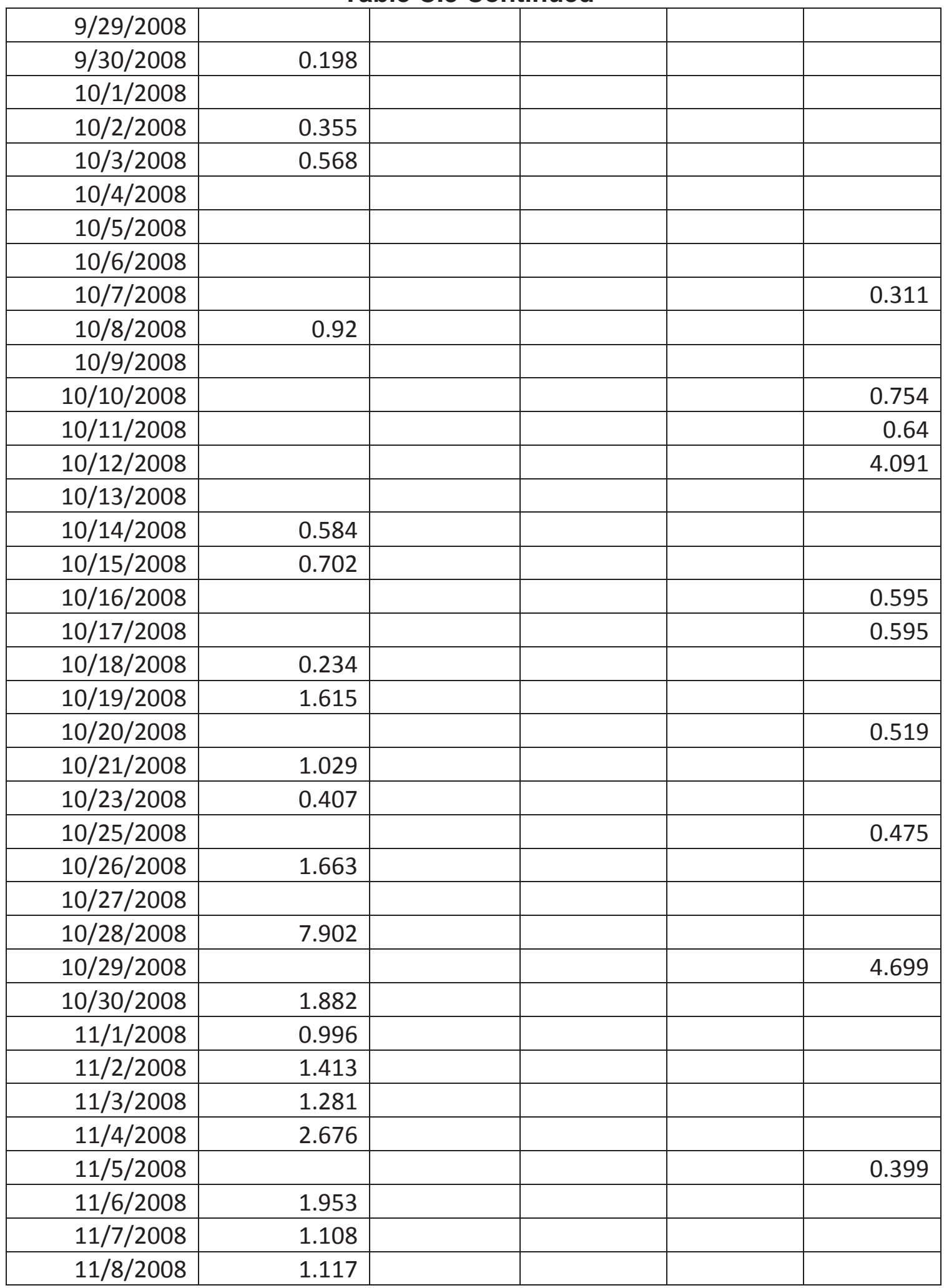


Table C.3 Continued

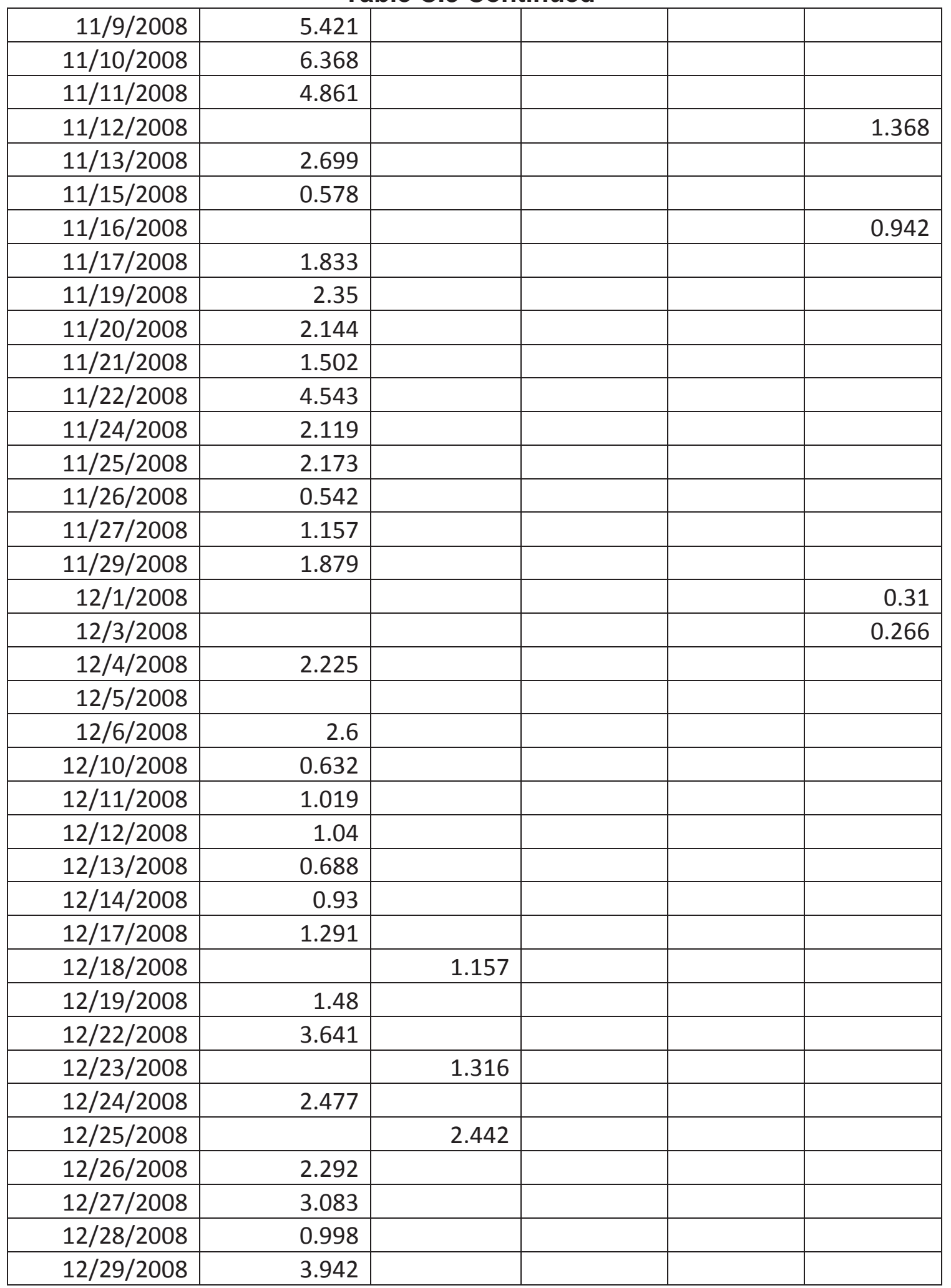


Table C.3 Continued

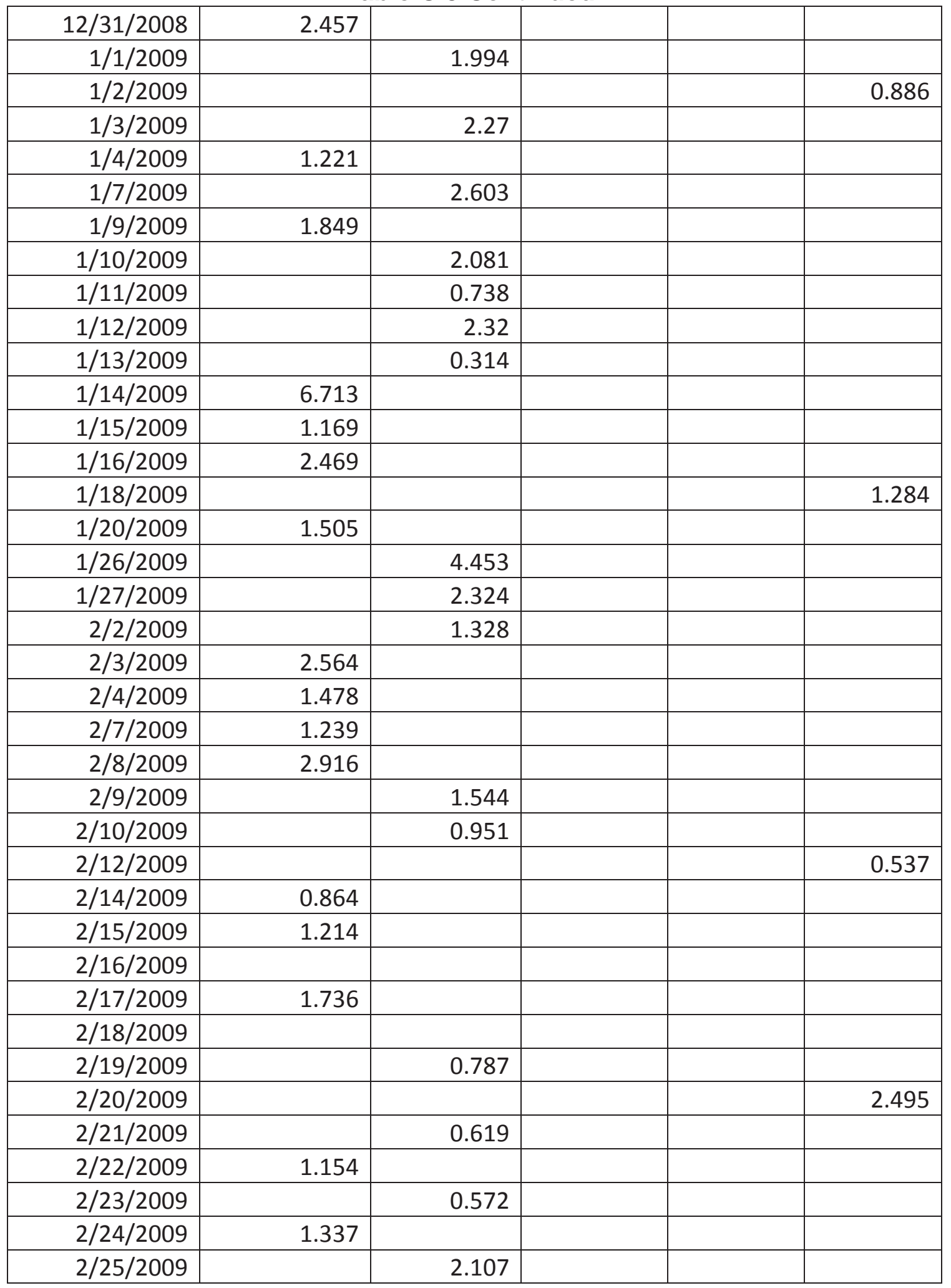


Table C.3 Continued

\begin{tabular}{|c|c|c|c|c|}
\hline $2 / 26 / 2009$ & & 0.888 & & \\
\hline $2 / 28 / 2009$ & & & & 1.082 \\
\hline $3 / 7 / 2009$ & & & & 0.477 \\
\hline $3 / 10 / 2009$ & 1.656 & & & \\
\hline $3 / 11 / 2009$ & & & & 0.574 \\
\hline $3 / 12 / 2009$ & & & & 0.352 \\
\hline $3 / 13 / 2009$ & 0.152 & & & \\
\hline $3 / 14 / 2009$ & 0.333 & & & \\
\hline $3 / 23 / 2009$ & & & & 0.404 \\
\hline $3 / 24 / 2009$ & 1.191 & & & \\
\hline $3 / 25 / 2009$ & & & & 0.327 \\
\hline $3 / 26 / 2009$ & 1.576 & & & \\
\hline $4 / 1 / 2009$ & & & & 0.289 \\
\hline $4 / 2 / 2009$ & 0.904 & & & \\
\hline $4 / 3 / 2009$ & & & & 0.297 \\
\hline $4 / 4 / 2009$ & 0.507 & & & \\
\hline $4 / 8 / 2009$ & & & & 0.111 \\
\hline $4 / 9 / 2009$ & & & & \\
\hline $4 / 10 / 2009$ & & & & 0.244 \\
\hline $4 / 11 / 2009$ & 1.49 & & & \\
\hline $4 / 14 / 2009$ & & 0.462 & & \\
\hline $4 / 16 / 2009$ & & & & 1.349 \\
\hline $4 / 17 / 2009$ & & & & 0.73 \\
\hline $4 / 18 / 2009$ & 1.86 & & & \\
\hline $4 / 24 / 2009$ & & & & 0.471 \\
\hline $4 / 27 / 2009$ & 2.194 & & & \\
\hline $5 / 1 / 2009$ & & & 1.109 & \\
\hline $5 / 5 / 2009$ & 0.728 & & & \\
\hline $5 / 17 / 2009$ & & & 0.463 & \\
\hline $5 / 20 / 2009$ & 2.876 & & & \\
\hline $5 / 24 / 2009$ & & 0.684 & & \\
\hline $5 / 26 / 2009$ & & 0.536 & & \\
\hline $5 / 28 / 2009$ & & 0.758 & & \\
\hline $6 / 2 / 2009$ & & 0.912 & & \\
\hline $6 / 9 / 2009$ & & 1.346 & & \\
\hline $6 / 10 / 2009$ & & 1.031 & & \\
\hline $6 / 11 / 2009$ & 0.883 & & & \\
\hline $6 / 12 / 2009$ & & 0.523 & & \\
\hline
\end{tabular}


Table C.3 Continued

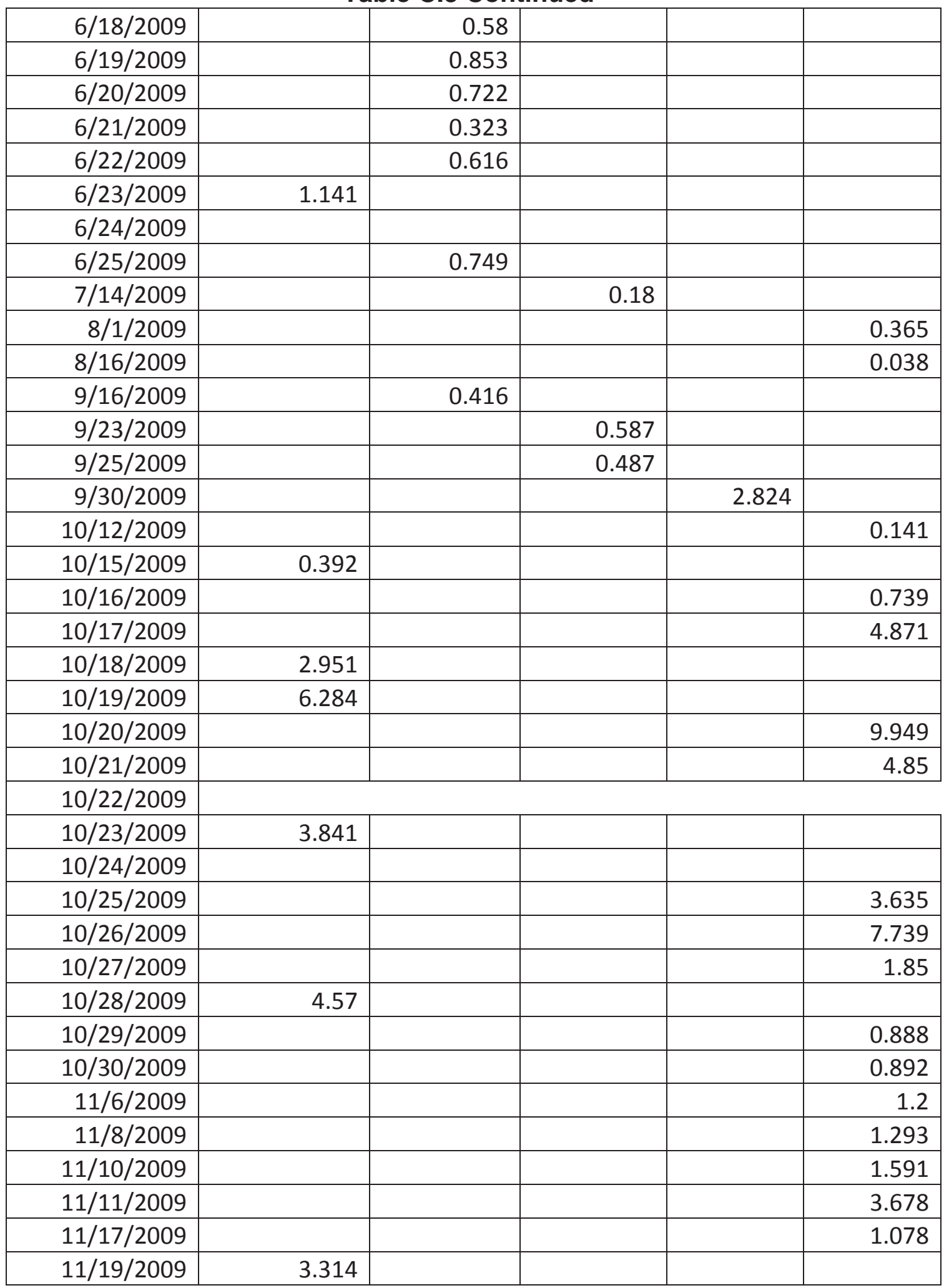


Table C.3 Continued

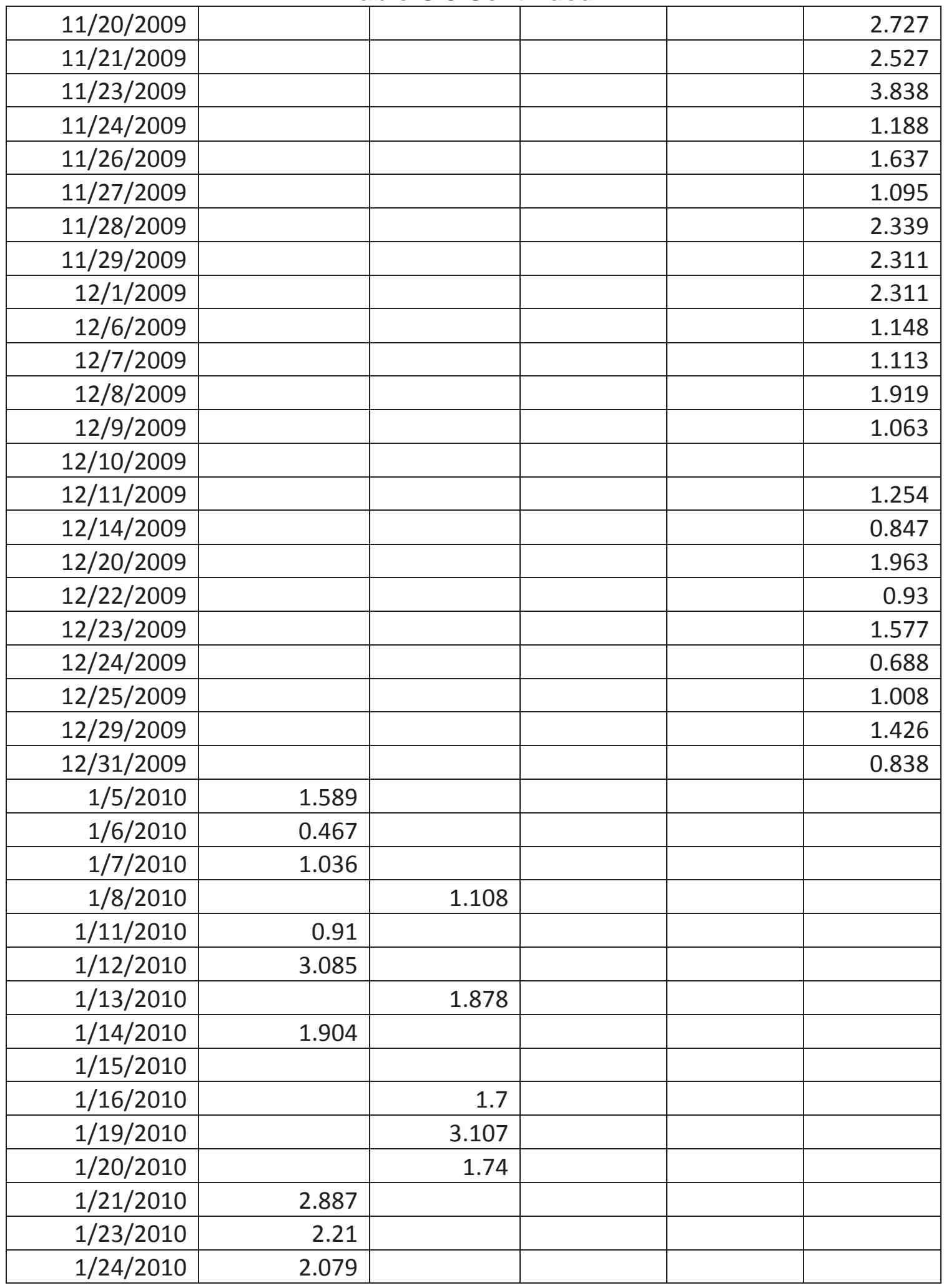


Table C.3 Continued

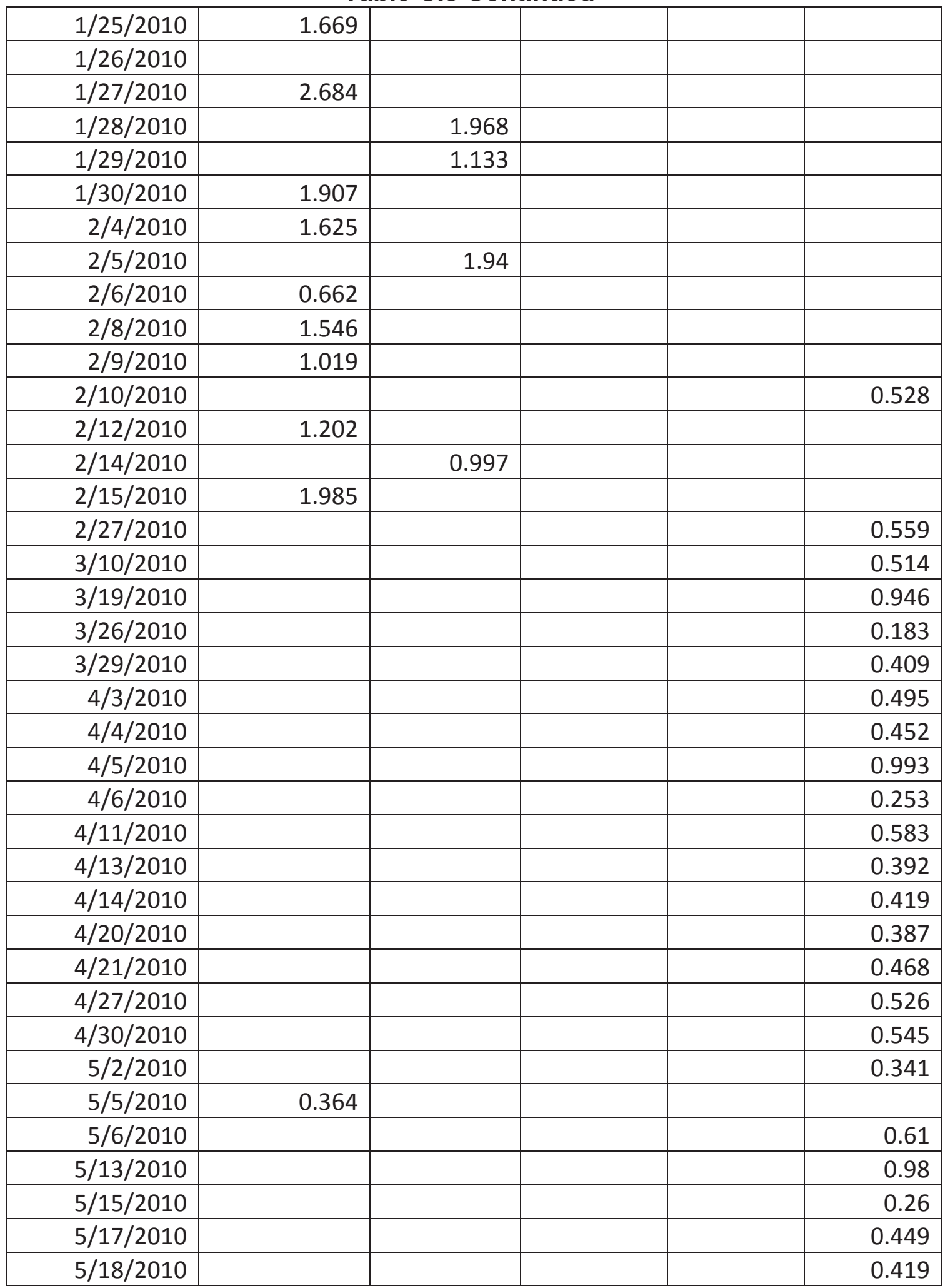


Table C.3 Continued

\begin{tabular}{|c|c|c|c|c|}
\hline $5 / 24 / 2010$ & & & & 0.576 \\
\hline $5 / 28 / 2010$ & 6.688 & & & \\
\hline $5 / 29 / 2010$ & & & 1.143 & \\
\hline $5 / 31 / 2010$ & & & & 0.72 \\
\hline $6 / 1 / 2010$ & 1.882 & & & \\
\hline $6 / 2 / 2010$ & 1.233 & & & \\
\hline $6 / 4 / 2010$ & & 0.946 & & \\
\hline $6 / 5 / 2010$ & & 1.209 & & \\
\hline $6 / 6 / 2010$ & & 0.959 & & \\
\hline $6 / 7 / 2010$ & & 0.621 & & \\
\hline $6 / 12 / 2010$ & & 0.767 & & \\
\hline $6 / 24 / 2010$ & & & & 0.575 \\
\hline $7 / 3 / 2010$ & 0.703 & & & \\
\hline $7 / 9 / 2010$ & 0.588 & & & \\
\hline $7 / 21 / 2010$ & & & & 0.166 \\
\hline $7 / 22 / 2010$ & 0.619 & & & \\
\hline $8 / 1 / 2010$ & & & & 0.47 \\
\hline $8 / 2 / 2010$ & 0.694 & & & \\
\hline $8 / 3 / 2010$ & & & & 0.174 \\
\hline $8 / 4 / 2010$ & & & & 1.442 \\
\hline $8 / 10 / 2010$ & & & & 0.411 \\
\hline $8 / 12 / 2010$ & & & & 0.076 \\
\hline $8 / 17 / 2010$ & 0.574 & & & \\
\hline $8 / 18 / 2010$ & 0.583 & & & \\
\hline $8 / 19 / 2010$ & & & & 0.425 \\
\hline $8 / 20 / 2010$ & & & & 1.955 \\
\hline $8 / 21 / 2010$ & & & & 0.18 \\
\hline $8 / 26 / 2010$ & & & & 0.489 \\
\hline $8 / 27 / 2010$ & & & & 0.889 \\
\hline $8 / 28 / 2010$ & & & & 0.287 \\
\hline $9 / 1 / 2010$ & & & & 0.931 \\
\hline $9 / 2 / 2010$ & & & & 1.89 \\
\hline $9 / 4 / 2010$ & & & & 0.61 \\
\hline $9 / 5 / 2010$ & & & & 1.395 \\
\hline $9 / 6 / 2010$ & & & & 0.116 \\
\hline $9 / 7 / 2010$ & & & & 0.386 \\
\hline $9 / 10 / 2010$ & & & & 0.314 \\
\hline $9 / 11 / 2010$ & & & & 0.699 \\
\hline
\end{tabular}


Table C.3 Continued

\begin{tabular}{|c|c|c|c|}
\hline $9 / 12 / 2010$ & & & 0.824 \\
\hline $9 / 13 / 2010$ & & & 1.187 \\
\hline $9 / 18 / 2010$ & & & 0.484 \\
\hline $9 / 20 / 2010$ & & & 0.39 \\
\hline $9 / 21 / 2010$ & & & 3.719 \\
\hline $9 / 23 / 2010$ & & & 0.387 \\
\hline $9 / 24 / 2010$ & & & 0.406 \\
\hline $9 / 26 / 2010$ & & & 0.812 \\
\hline $9 / 28 / 2010$ & 1.084 & & \\
\hline 9/29/2010 & & & 0.427 \\
\hline $10 / 4 / 2010$ & & & 0.284 \\
\hline $10 / 5 / 2010$ & 0.288 & & \\
\hline $10 / 7 / 2010$ & 1.261 & & \\
\hline $10 / 13 / 2010$ & 0.366 & & \\
\hline $10 / 14 / 2010$ & 0.147 & & \\
\hline $10 / 22 / 2010$ & & & 0.554 \\
\hline $10 / 29 / 2010$ & & & 0.637 \\
\hline $10 / 31 / 2010$ & & & 0.144 \\
\hline $11 / 7 / 2010$ & & & 0.306 \\
\hline $11 / 23 / 2010$ & & & 0.229 \\
\hline $11 / 25 / 2010$ & 0.913 & & \\
\hline $11 / 29 / 2010$ & & 0.753 & \\
\hline $12 / 2 / 2010$ & & 0.827 & \\
\hline $12 / 3 / 2010$ & & 0.746 & \\
\hline $12 / 5 / 2010$ & & 4.693 & \\
\hline $12 / 6 / 2010$ & & 1.049 & \\
\hline $12 / 9 / 2010$ & & 0.853 & \\
\hline $12 / 13 / 2010$ & & 0.616 & \\
\hline $12 / 16 / 2010$ & 0.812 & & \\
\hline $12 / 25 / 2010$ & & 0.588 & \\
\hline $12 / 26 / 2010$ & & 0.055 & \\
\hline
\end{tabular}




\section{Copyright Permissions}

\section{Copyright permission for Figure 4.2}

\section{Geological Society of London}

\section{Re-publishing material from Geological Society of London copyright}

\section{Using material from GSL publications}

Extensive quotation (more than c. 100 words) and reproduction of previously published illustrations (even if redrawn) require permission from the copyright holder. In the UK, material remains in copyright until 70 years after the death of the author.

\section{Material that can be used without permission}

Authors may reuse their own material without permission subject to the exceptions listed below. They may include the whole article in a $\mathrm{PhD}$ or other thesis provided that it will not be published, and that the original source is fully acknowledged in the standard form. Authors may not republish their whole article, or a substantial part of it, without permission. Such permission will be granted only in exceptional circumstances.

Anyone may use up to three items (text extracts of 100 words or less, figures or tables) from GSL published material without permission or charge provided that a proper acknowledgement of source is used with the item. If you require written permission, please use the forms in the section below; note that payment will be required.

The abstracts of articles can be reproduced without permission or fees provided that a full reference and a link to the article abstract page are included.

Please check that GSL is the original copyright holder and that the material has not been taken from another source. In those cases, you must contact the original copyright holder. GSL is not usually the copyright holder for cover photographs, and permission must be sought from the copyright holder.

If your article forms part of a multi-author book, the publisher must ensure that the total number of items from GSL copyright does not exceed three (this does not apply to journal issues).

\section{Reusing material in another GSL publication}

You may use material from GSL copyright in another GSL publication without permission from GSL subject to the exceptions listed below. However, if there are more 
than three items from an individual article, you must seek the author's consent directly. If he/she does not respond within four weeks, you can assume that consent is given.

\section{Uses where permission is required}

For more than three items, please use Rightslink to obtain permission; there will be a charge of $\$ 25$ per item (NB: GSL Fellows are entitled to a discount and should use the form below).

If there are more than three items from an individual article, you must also seek the author's consent. If he/she does not respond within four weeks, you can assume consent. You should contact the author at the address on the original article, or use Google Scholar or other search engine to trace them. Authors are responsible for ensuring that their mail gets forwarded when they move. For papers published before 1980, a more pragmatic approach can be taken with author consent. If you want GSL to check the authors' contact details there may be a charge for the service and there is no guarantee that we have the correct address.

For Fellows of the Geological Society and those unable to use Rightslink, requests must be made using the form below.

Current fees for republishing material from GSL Copyright;

- $\quad$ Processing fee per request: $£ 10$ for e-mail requests and replies; $£ 20$ when a printed letter is required.

- Item fee for each figure, table or extract: $£ 15$ per item (there are no free items if written permission is needed)

- $50 \%$ discount for GSL Fellows

- VAT at the standard rate of $20 \%$

\section{Application Form}

- $\quad$ Please download and complete the request form (.rtf130 Kb)

\section{Exceptions}

The Geological Society of London has a strong policy against duplicate publication and does not allow republication of whole articles, or substantial parts of them, unless there are very exceptional circumstances.

You may not use more than $25 \%$ of any GSL published article in a single publication and no publication should contain more than $25 \%$ in total from GSL Copyright. This does not prevent the inclusion of whole articles in course packs and the like (which do not constitute republication). 
You may not post a typeset PDF of a GSL article on any website, repository or server (see Lyell Collection terms and conditions for more information).

For enquiries please contact Angharad.Hills@geolsoc.org.uk

\section{Photocopying and digital copying}

You may make limited hard copies of an article for your own research or educational purposes (not including course packs) if you have access rights to the material. You do not need to request permission to do this.

For more extensive copying or digital reproduction, you need to obtain a licence from the Copyright Licensing Agency: www.cla.co.uk or the Copyright Clearance Center: www.copyright.com or from your local reproduction rights agency.

\section{Lecture and presentations}

You may include material from Society copyright in a visual presentation if you have access rights to the material and provided that you will not circulate digital or hard copies of your presentation. You do not need to request permission to do this. If you wish to circulate copies of your presentation to students or others, please obtain 'coursepack' permission from the Copyright Licensing Agency: www.cla.co.uk or the Copyright Clearance Center: www.copyright.com or from your local reproduction rights agency.

\section{Using other GSL material}

Permission to reprint material from the Society's unpublished archives/art collection should be made to the Society's Archivist.

Permission to use material from the Society's website should be made to Sara Anders. Permission to use material from the Geoscientist magazine should be made to $\underline{\text { Ted }}$ Nield. 


\section{Copyright permission for Chapter 2}

This is a License Agreement between Lorna A Henney ("You") and Springer ("Springer") provided by Copyright Clearance Center ("CCC"). The license consists of your order details, the terms and conditions provided by Springer, and the payment terms and conditions.

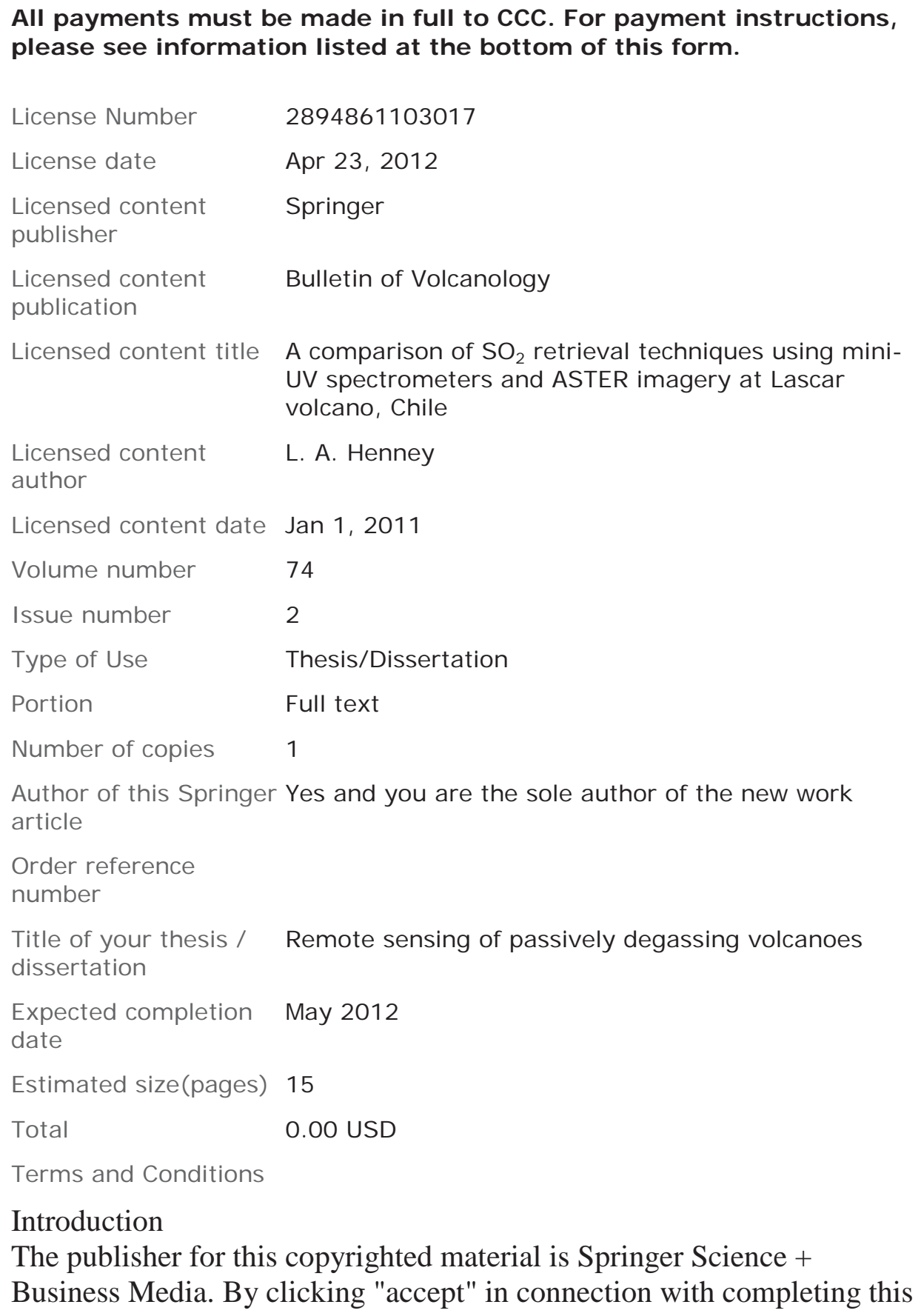

Terms and Conditions

Introduction

The publisher for this copyrighted material is Springer Science + Business Media. By clicking "accept" in connection with completing this 
licensing transaction, you agree that the following terms and conditions apply to this transaction (along with the Billing and Payment terms and conditions established by Copyright Clearance Center, Inc. ("CCC"), at the time that you opened your Rightslink account and that are available at any time at http://myaccount.copyright.com).

\section{Limited License}

With reference to your request to reprint in your thesis material on which Springer Science and Business Media control the copyright, permission is granted, free of charge, for the use indicated in your enquiry.

Licenses are for one-time use only with a maximum distribution equal to the number that you identified in the licensing process.

This License includes use in an electronic form, provided its password protected or on the university's intranet or repository, including UMI (according to the definition at the Sherpa website:

http://www.sherpa.ac.uk/romeo/). For any other electronic use, please contact Springer at (permissions.dordrecht@ springer.com or permissions.heidelberg@springer.com).

The material can only be used for the purpose of defending your thesis, and with a maximum of 100 extra copies in paper.

Although Springer holds copyright to the material and is entitled to negotiate on rights, this license is only valid, provided permission is also obtained from the (co) author (address is given with the article/chapter) and provided it concerns original material which does not carry references to other sources (if material in question appears with credit to another source, authorization from that source is required as well).

Permission free of charge on this occasion does not prejudice any rights we might have to charge for reproduction of our copyrighted material in the future.

Altering/Modifying Material: Not Permitted You may not alter or modify the material in any manner. Abbreviations, additions, deletions and/or any other alterations shall be made only with prior written authorization of the author(s) and/or Springer Science + Business Media. (Please contact Springer at (permissions.dordrecht@springer.com or permissions.heidelberg@springer.com)

Reservation of Rights Springer Science + Business Media reserves all rights not specifically 
granted in the combination of (i) the license details provided by you and accepted in the course of this licensing transaction, (ii) these terms and conditions and (iii) CCC's Billing and Payment terms and conditions.

Copyright Notice:Disclaimer

You must include the following copyright and permission notice in connection with any reproduction of the licensed material: "Springer and the original publisher /journal title, volume, year of publication, page, chapter/article title, name(s) of author(s), figure number(s), original copyright notice) is given to the publication in which the material was originally published, by adding; with kind permission from Springer Science and Business Media"

Warranties: None

Example 1: Springer Science + Business Media makes no representations or warranties with respect to the licensed material.

Example 2: Springer Science + Business Media makes no representations or warranties with respect to the licensed material and adopts on its own behalf the limitations and disclaimers established by CCC on its behalf in its Billing and Payment terms and conditions for this licensing transaction.

Indemnity You hereby indemnify and agree to hold harmless Springer Science + Business Media and CCC, and their respective officers, directors, employees and agents, from and against any and all claims arising out of your use of the licensed material other than as specifically authorized pursuant to this license.

No Transfer of License

This license is personal to you and may not be sublicensed, assigned, or transferred by you to any other person without Springer Science + Business Media's written permission.

No Amendment Except in Writing

This license may not be amended except in a writing signed by both parties (or, in the case of Springer Science + Business Media, by CCC on Springer Science + Business Media's behalf).

Objection to Contrary Terms

Springer Science + Business Media hereby objects to any terms contained in any purchase order, acknowledgment, check endorsement or other writing prepared by you, which terms are inconsistent with these 
terms and conditions or CCC's Billing and Payment terms and conditions. These terms and conditions, together with CCC's Billing and Payment terms and conditions (which are incorporated herein), comprise the entire agreement between you and Springer Science + Business Media (and CCC) concerning this licensing transaction. In the event of any conflict between your obligations established by these terms and conditions and those established by CCC's Billing and Payment terms and conditions, these terms and conditions shall control.

\section{Jurisdiction}

All disputes that may arise in connection with this present License, or the breach thereof, shall be settled exclusively by arbitration, to be held in The Netherlands, in accordance with Dutch law, and to be conducted under the Rules of the 'Netherlands Arbitrage Instituut' (Netherlands Institute of Arbitration).OR:

All disputes that may arise in connection with this present License, or the breach thereof, shall be settled exclusively by arbitration, to be held in the Federal Republic of Germany, in accordance with German law.

Other terms and conditions:

\section{$\mathbf{v 1 . 3}$}

If you would like to pay for this license now, please remit this license along with your payment made payable to "COPYRI GHT CLEARANCE CENTER" otherwise you will be invoiced within 48 hours of the license date. Payment should be in the form of a check or money order referencing your account number and this invoice number RLNK500766183.

Once you receive your invoice for this order, you may pay your invoice by credit card. Please follow instructions provided at that time.

Make Payment To:

Copyright Clearance Center

Dept 001

P.O. Box 843006

Boston, MA 02284-3006

For suggestions or comments regarding this order, contact RightsLink Customer Support: customercare@copyright.com or +1-877-6225543 (toll free in the US) or +1-978-646-2777.

Gratis licenses (referencing $\$ 0$ in the Total field) are free. Please retain this printable license for your reference. No payment is required. 


\section{Copyright permission for Figure 4.5.}

This is a License Agreement between Lorna A Henney ("You") and Elsevier ("Elsevier") provided by Copyright Clearance Center ("CCC"). The license consists of your order details, the terms and conditions provided by Elsevier, and the payment terms and conditions.

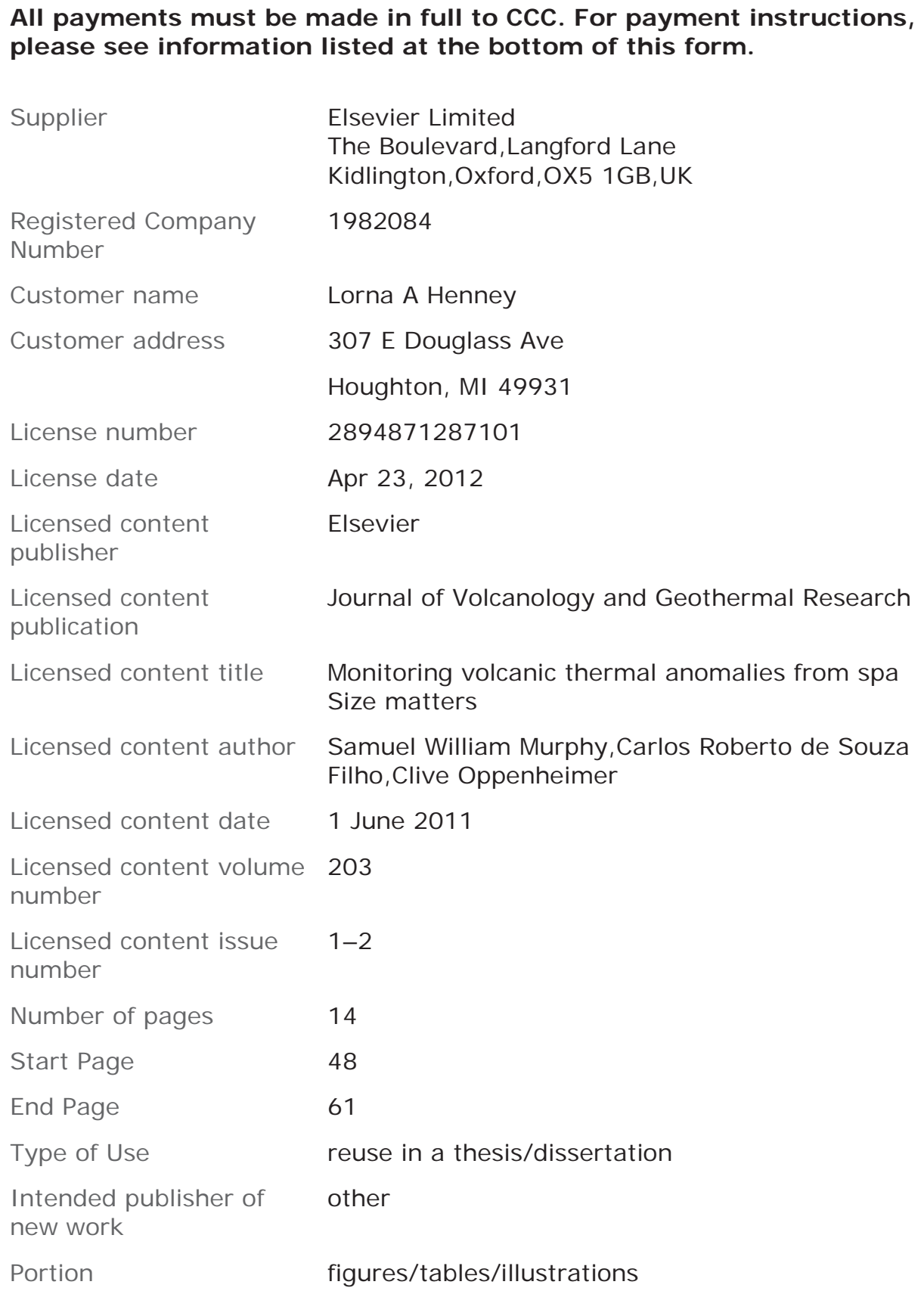




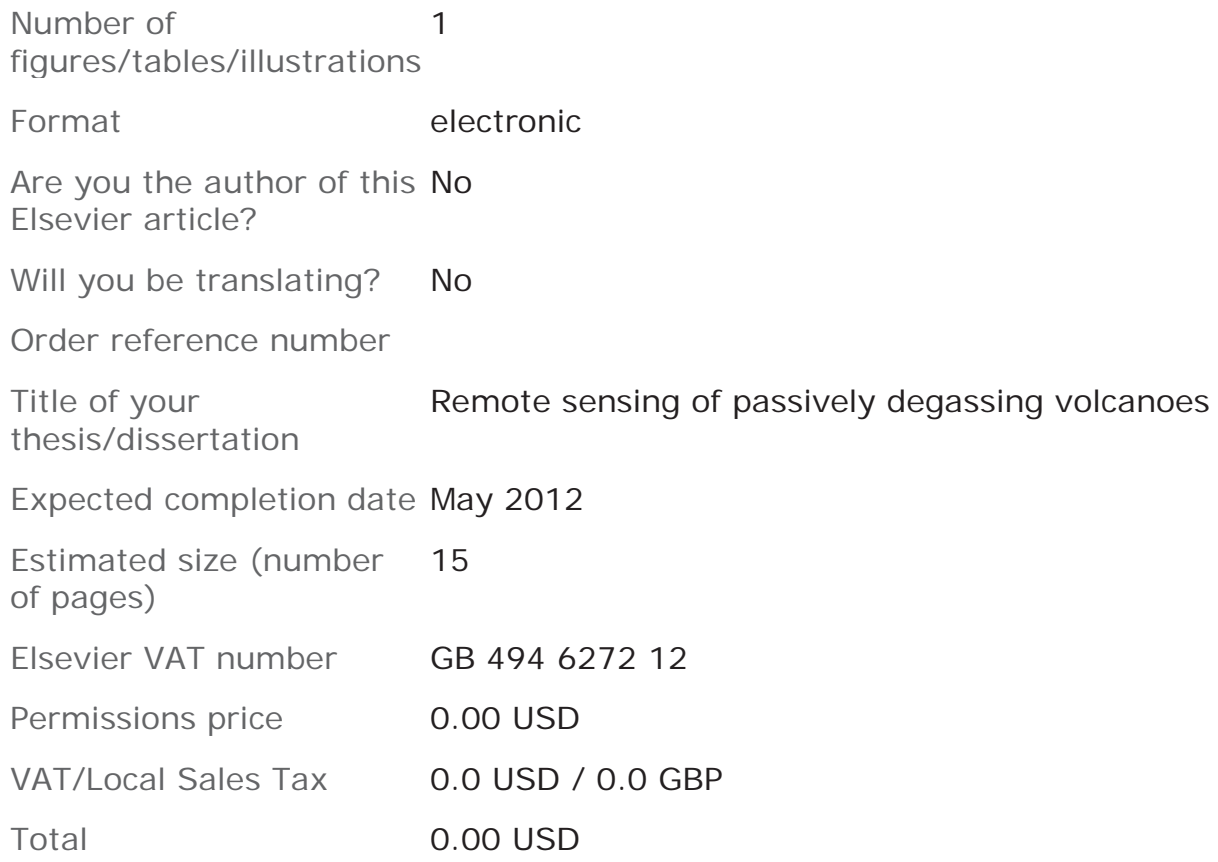

Terms and Conditions

\section{INTRODUCTION}

1. The publisher for this copyrighted material is Elsevier. By clicking "accept" in connection with completing this licensing transaction, you agree that the following terms and conditions apply to this transaction (along with the Billing and Payment terms and conditions established by Copyright Clearance Center, Inc. ("CCC"), at the time that you opened your Rightslink account and that are available at any time at http://myaccount.copyright.com).

\section{GENERAL TERMS}

2. Elsevier hereby grants you permission to reproduce the aforementioned material subject to the terms and conditions indicated.

3. Acknowledgement: If any part of the material to be used (for example, figures) has appeared in our publication with credit or acknowledgement to another source, permission must also be sought from that source. If such permission is not obtained then that material may not be included in your publication/copies. Suitable acknowledgement to the source must be made, either as a footnote or in a reference list at the end of your publication, as follows:

"Reprinted from Publication title, Vol /edition number, Author(s), Title of article / title of chapter, Pages No., Copyright (Year), with permission 
from Elsevier [OR APPLICABLE SOCIETY COPYRIGHT OWNER]." Also Lancet special credit - "Reprinted from The Lancet, Vol. number, Author(s), Title of article, Pages No., Copyright (Year), with permission from Elsevier."

4. Reproduction of this material is confined to the purpose and/or media for which permission is hereby given.

5. Altering/Modifying Material: Not Permitted. However figures and illustrations may be altered/adapted minimally to serve your work. Any other abbreviations, additions, deletions and/or any other alterations shall be made only with prior written authorization of Elsevier Ltd. (Please contact Elsevier at permissions@elsevier.com)

6. If the permission fee for the requested use of our material is waived in this instance, please be advised that your future requests for Elsevier materials may attract a fee.

7. Reservation of Rights: Publisher reserves all rights not specifically granted in the combination of (i) the license details provided by you and accepted in the course of this licensing transaction, (ii) these terms and conditions and (iii) CCC's Billing and Payment terms and conditions.

8. License Contingent Upon Payment: While you may exercise the rights licensed immediately upon issuance of the license at the end of the licensing process for the transaction, provided that you have disclosed complete and accurate details of your proposed use, no license is finally effective unless and until full payment is received from you (either by publisher or by (CC) as provided in CCC's Billing and Payment terms and conditions. If full payment is not received on a timely basis, then any license preliminarily granted shall be deemed automatically revoked and shall be void as if never granted. Further, in the event that you breach any of these terms and conditions or any of CCC's Billing and Payment terms and conditions, the license is automatically revoked and shall be void as if never granted. Use of materials as described in a revoked license, as well as any use of the materials beyond the scope of an unrevoked license, may constitute copyright infringement and publisher reserves the right to take any and all action to protect its copyright in the materials.

9. Warranties: Publisher makes no representations or warranties with respect to the licensed material.

10. Indemnity: You hereby indemnify and agree to hold harmless publisher and CCC, and their respective officers, directors, employees 
and agents, from and against any and all claims arising out of your use of the licensed material other than as specifically authorized pursuant to this license.

11. No Transfer of License: This license is personal to you and may not be sublicensed, assigned, or transferred by you to any other person without publisher's written permission.

12. No Amendment Except in Writing: This license may not be amended except in a writing signed by both parties (or, in the case of publisher, by $\mathrm{CCC}$ on publisher's behalf).

13. Objection to Contrary Terms: Publisher hereby objects to any terms contained in any purchase order, acknowledgment, check endorsement or other writing prepared by you, which terms are inconsistent with these terms and conditions or CCC's Billing and Payment terms and conditions. These terms and conditions, together with CCC's Billing and Payment terms and conditions (which are incorporated herein), comprise the entire agreement between you and publisher (and CCC) concerning this licensing transaction. In the event of any conflict between your obligations established by these terms and conditions and those established by CCC's Billing and Payment terms and conditions, these terms and conditions shall control.

14. Revocation: Elsevier or Copyright Clearance Center may deny the permissions described in this License at their sole discretion, for any reason or no reason, with a full refund payable to you. Notice of such denial will be made using the contact information provided by you. Failure to receive such notice will not alter or invalidate the denial. In no event will Elsevier or Copyright Clearance Center be responsible or liable for any costs, expenses or damage incurred by you as a result of a denial of your permission request, other than a refund of the amount(s) paid by you to Elsevier and/or Copyright Clearance Center for denied permissions.

\section{LIMITED LICENSE}

The following terms and conditions apply only to specific license types:

15. Translation: This permission is granted for non-exclusive world English rights only unless your license was granted for translation rights. If you licensed translation rights you may only translate this content into the languages you requested. A professional translator must perform all translations and reproduce the content word for word preserving the integrity of the article. If this license is to re-use 1 or 2 
figures then permission is granted for non-exclusive world rights in all languages.

16. Website: The following terms and conditions apply to electronic reserve and author websites:

Electronic reserve: If licensed material is to be posted to website, the web site is to be password-protected and made available only to bona fide students registered on a relevant course if:

This license was made in connection with a course, This permission is granted for 1 year only. You may obtain a license for future website posting,

All content posted to the web site must maintain the copyright information line on the bottom of each image, A hyper-text must be included to the Homepage of the journal from which you are licensing at http://www.sciencedirect.com/science/journal/xxxxx or the Elsevier homepage for books at http://www.elsevier.com, and

Central Storage: This license does not include permission for a scanned version of the material to be stored in a central repository such as that provided by Heron/XanEdu.

17. Author website for journals with the following additional clauses:

All content posted to the web site must maintain the copyright information line on the bottom of each image, and he permission granted is limited to the personal version of your paper. You are not allowed to download and post the published electronic version of your article (whether PDF or HTML, proof or final version), nor may you scan the printed edition to create an electronic version, A hyper-text must be included to the Homepage of the journal from which you are licensing at http://www.sciencedirect.com/science/journal/xxxxx, As part of our normal production process, you will receive an e-mail notice when your article appears on Elsevier's online service ScienceDirect (www.sciencedirect.com). That e-mail will include the article's Digital Object Identifier (DOI). This number provides the electronic link to the published article and should be included in the posting of your personal version. We ask that you wait until you receive this e-mail and have the DOI to do any posting.

Central Storage: This license does not include permission for a scanned version of the material to be stored in a central repository such as that provided by Heron/XanEdu.

18. Author website for books with the following additional clauses: Authors are permitted to place a brief summary of their work online 
only.

A hyper-text must be included to the Elsevier homepage at http://www.elsevier.com

All content posted to the web site must maintain the copyright information line on the bottom of each image

You are not allowed to download and post the published electronic version of your chapter, nor may you scan the printed edition to create an electronic version.

Central Storage: This license does not include permission for a scanned version of the material to be stored in a central repository such as that provided by Heron/XanEdu.

19. Website (regular and for author): A hyper-text must be included to the Homepage of the journal from which you are licensing at http://www.sciencedirect.com/science/journal/xxxxx. or for books to the Elsevier homepage at http://www.elsevier.com

20. Thesis/Dissertation: If your license is for use in a thesis/dissertation your thesis may be submitted to your institution in either print or electronic form. Should your thesis be published commercially, please reapply for permission. These requirements include permission for the Library and Archives of Canada to supply single copies, on demand, of the complete thesis and include permission for UMI to supply single copies, on demand, of the complete thesis. Should your thesis be published commercially, please reapply for permission.

\section{Other Conditions:}

v1.6

If you would like to pay for this license now, please remit this license along with your payment made payable to "COPYRI GHT CLEARANCE CENTER" otherwise you will be invoiced within 48 hours of the license date. Payment should be in the form of a check or money order referencing your account number and this invoice number RLNK500766195.

Once you receive your invoice for this order, you may pay your invoice by credit card. Please follow instructions provided at that time.

Make Payment To:

Copyright Clearance Center

Dept 001

P.O. Box 843006

Boston, MA 02284-3006 
For suggestions or comments regarding this order, contact

RightsLink Customer Support: customercare@copyright.com or +1-

877-622-5543 (toll free in the US) or +1-978-646-2777.

Gratis licenses (referencing $\$ 0$ in the Total field) are free. Please retain this printable license for your reference. No payment is required. 